\title{
Subálgebras de Mishchenko-Fomenko de álgebras envolventes de álgebras de Lie simples
}

\author{
Maria Clara Cardoso
}

\author{
DisSERTAÇÃO APRESENTADA \\ $\mathrm{AO}$ \\ Instituto DE MatemáticA E Estatística \\ DA \\ Universidade DE SÃo PaUlo \\ PARA \\ OBTENÇÃO DO TÍTULO \\ DE \\ Mestre EM CiÊnCIAS
}

Programa: Matemática

Orientador: Prof. Dr. Vyacheslav Futorny

Durante o desenvolvimento deste trabalho o autor recebeu auxílio financeiro do CNPq e da FAPESP (processo: 2017/11050-0)

São Paulo, agosto de 2019 


\section{Subálgebras de Mishchenko-Fomenko de álgebras envolventes de álgebras de Lie simples}

Esta versão da dissertação contém as correções e alterações sugeridas pela Comissão Julgadora durante a defesa da versão original do trabalho, realizada em 02/08/2019. Uma cópia da versão original está disponível no

Instituto de Matemática e Estatística da Universidade de São Paulo.

Comissão Julgadora:

- Prof. Dr. Vyacheslav Futorny (orientador) - IME-USP

- Prof. Dr. Plamen Emilov Kochloukov - UNICAMP

- Prof. Dr. Lucas Henrique Calixto - UFMG 


\section{Resumo}

\section{CARDOSO, M. C. Subálgebras de Mishchenko-Fomenko de álgebras envolventes de álge-}

bras de Lie simples. 2019. 94 f. Dissertação (Mestrado) - Instituto de Matemática e Estatística, Universidade de São Paulo, São Paulo, 2019.

Nesse trabalho introduzimos as subálgebras de Mishchenko-Fomenko. Apresentamos o problema de Vinberg e a solução de Feigin, Frenkel e Toledano-Laredo Feigin et al. [2010]. Também é mostrada a solução para as álgebras de Lie de tipo A apresentada em Futorny e Molev [2015]. É estudado também o artigo Molev [2013] onde são apresentados geradores do centro de Feigin-Frenkel para as álgebras de Lie de tipo B, C e D. Também são introduzidas as subálgebras de GelfandTsetlin, subálgebras das álgebras envolventes universais das álgebras de Lie de tipo A. Apresentamos uma definição de súbálgebra de Gelfand-Tsetlin para as álgebras de Lie de tipo C, introduzida em Molev e Yakimova [2017]. São exibidas as variedades de Gelfand-Tsetlin de $\mathfrak{s p}_{4}$ e $\mathfrak{s p}_{6}$, sendo provado que a variedade de Gelfand-Tsetlin de $\mathfrak{s p}_{4}$ é equidimensional de dimensão 4 . Também é demonstrado um novo resultado sobre a equidimensionalidade de $\mathfrak{s p}_{6}$.

Palavras-chave: subálgebra de Mishchenko-Fomenko, problema de Vinberg, variedade de GelfandTsetlin. 


\section{Abstract}

CARDOSO, M. C. Mishchenko-Fomenko subalgebras of universal enveloping algebras of simple Lie algebras. 2019. 94 f. Dissertação (Mestrado) - Instituto de Matemática e Estatística, Universidade de São Paulo, São Paulo, 2019.

In this dissertation, we introduce the Mishchenko-Fomenko subalgebras. We show Vinberg's problem and the solution given by Feigin, Frenkel and Toledano-Laredo Feigin et al. [2010]. We also show a solution for Lie algebras of type A found in Futorny e Molev [2015]. We study the article Molev [2013] where generators for the Feigin-Frenkel center are shown for Lie algebras of type B, C and D. We introduce the Gelfand-Tsetlin subalgebras, which are subalgebras of the universal enveloping algebras of Lie algebras of type A. We show a definition of Gelfand-Tsetlin for Lie algebras of type C, introduced in Molev e Yakimova [2017]. We exhibit the Gelfand-Tsetlin varieties related to $\mathfrak{s p}_{4}$ and $\mathfrak{s p}_{6}$. We prove that the Gelfand-Tsetlin variety for $\mathfrak{s p}_{4}$ is equidimensional of dimension 4 and we prove a new result about the equidimensionality of $\mathfrak{s p}_{6}$.

Keywords: Mishchenko-Fomenko subalgebras, Vinberg's problem, Gelfand-Tsetlin variety. 


\section{Sumário}

Lista de Símbolos $\quad$ vii

1 Introdução $\quad 1$

2 Conceitos preliminares $\quad 3$

2.1 Álgebra de Lie . . . . . . . . . . . . . . . . . . . . . . . 3

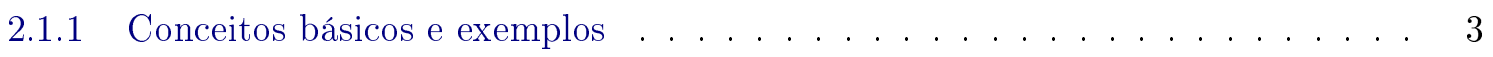

2.1.2 Representação e módulo . . . . . . . . . . . . . . . . . . . . . 4

2.1 .3 Álgebra de Lie semissimples . . . . . . . . . . . . . . . . . . 5

2.2 Álgebra graduada e álgebra filtrada . . . . . . . . . . . . . . . . . . 6

2.3 Álgebra tensorial . . . . . . . . . . . . . . . . . . . . . . . . . . . . . . . . . . .

2.3 .1 Álgebra simétrica . . . . . . . . . . . . . . . . . 7

2.3 .2 Álgebra exterior . . . . . . . . . . . . . . . . . 8

2.3 .3 Álgebra envolvente universal . . . . . . . . . . . . . . . . . . 9

2.4 Álgebra de Poisson-Lie . . . . . . . . . . . . . . . . . . . . . . . . . . . . . . . . . . . . . . . . . . . .

2.5 Álgebra homológica . . . . . . . . . . . . . . . . . . . . . . . 12

2.5.1 Complexo de Koszul . . . . . . . . . . . . . . . . . . . . 12

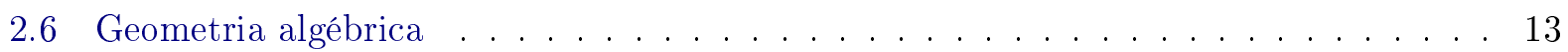

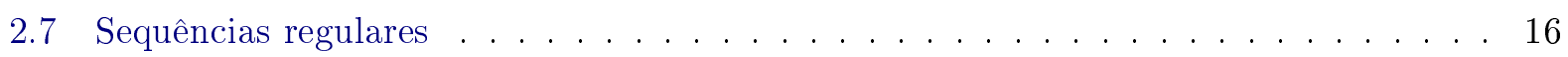

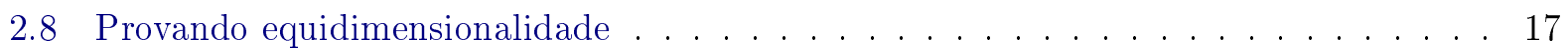

3 Subálgebra de Mishchenko-Fomenko $\quad 19$

3.1 Definição . . . . . . . . . . . . . . . . . . . . . . 19

3.2 Problema de Vinberg . . . . . . . . . . . . . . . . . . . . . . . 20

3.2.1 A solução de Feigin, Frenkel e Toledano-Laredo . . . . . . . . . . . . . . . 20

3.3 Alguns conceitos . . . . . . . . . . . . . . . . . . . 22

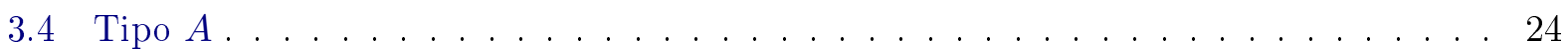

3.4.1 Geradores do centro de Feigin-Frenkel . . . . . . . . . . . . . . . . . . 24

3.4 .2 Geradores de $A_{\mu} \ldots \ldots \ldots \ldots \ldots \ldots$

3.4 .3 Geradores de $A_{\mu}$ algebricamente independentes . . . . . . . . . . . 31

3.5 Tipo B e D . . . . . . . . . . . . . . . . . . 33

3.5.1 Geradores do centro de Feigin-Frenkel . . . . . . . . . . . . . . . . . 33

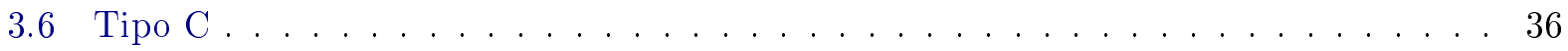

3.6.1 Geradores do centro de Feigin-Frenkel . . . . . . . . . . . . . . . . 36

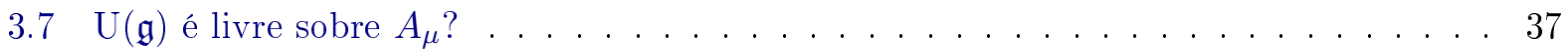


4 Subálgebras de Gelfand-Tsetlin 39

4.1 Definição . . . . . . . . . . . . . . . . . . . . 39

4.2 Relação com subálgebras de Mishchenko-Fomenko . . . . . . . . . . . . . . . . . . . 39

4.3 Subálgebras de Gelfand-Tsetlin para o tipo $C \ldots \ldots \ldots$. . . . . . . . . . 40

$\begin{array}{ll}\text { Apêndice } & 91\end{array}$

$\begin{array}{ll}\text { A Programas para calcular determinante } & 91\end{array}$

$\begin{array}{ll}\text { Referências Bibliográficas } & 93\end{array}$ 


\title{
Lista de Símbolos
}

\author{
$\mathbb{C}$ conjunto dos complexos \\ End endomorfismos \\ Im imagem de uma função \\ $k \quad$ corpo (normalmente fechado e de característica 0 ) \\ ker núcleo (kernel) de uma função \\ $S_{m} \quad$ grupo das permutações de $m$ elementos \\ sgn sinal de uma permutação \\ tr traço de uma matriz
}




\section{Capítulo 1}

\section{Introdução}

Representações de álgebras de Lie é uma das áreas mais importantes e ativas da teoria de representações com uma variedade ampla de resultados e aplicações. Seja $k$ um corpo de característica 0 e algebricamente fechado e seja $\mathfrak{g}$ uma $k$-álgebra de Lie. Podemos definir, a partir de $\mathfrak{g}$, uma álgebra associativa com unidade que chamamos de álgebra envolvente universal e denotamos por $\mathrm{U}(\mathfrak{g})$. É um fato conhecido que a categoria de $\mathfrak{g}$-módulos é equivalente a de $\mathrm{U}(\mathfrak{g})$-módulos. Assim podemos focar no estudo de $\mathrm{U}(\mathfrak{g})$-módulos.

Sejam $U$ uma $k$-álgebra associativa com 1 (por exemplo, $\mathrm{U}(\mathfrak{g})$ ) e $B$ uma subálgebra comutativa de $U$. Pode-se perguntar se um $B$-módulo irredutível pode ser levantado para um $U$-módulo irredutível. A resposta para essa pergunta é positiva se $U$ for um $B$-módulo livre (à esquerda ou à direita). Em Kostant [1963] é provado que U(g), onde $\mathfrak{g}$ é uma álgebra de Lie simples de dimensão finita, é um módulo livre (à esquerda ou à direita) sobre seu centro $Z$. Pelo resultado de Bernstein e Lunts [1996], temos que a álgebra polinomial $P(\mathfrak{g})=S\left(\mathfrak{g}^{*}\right)$ é livre sobre o conjunto de invariantes $P(\mathfrak{g})^{G}$, onde $G$ é o grupo de Lie correspondente a $\mathfrak{g}$.

Nesse contexto, as subálgebras de Mishchenko-Fomenko se mostram importantes. A álgebra envolvente universal possui uma filtração natural e a álgebra graduada associada a essa filtração é a álgebra simétrica $S(\mathfrak{g})$.

Definição 1.0.1. Sejam $\mathfrak{g}$ uma álgebra de Lie simples e $P\left(Y_{1}, \ldots, Y_{l}\right) \in S(\mathfrak{g})$ um elemento de grau $d$. Fixe $\mu \in \mathfrak{g}^{*}$. Seja $z$ uma variável. Substitua $Y_{i}$ por $Y_{i}+z \mu\left(Y_{i}\right)$ em $P$ e expanda o resultado para um polinômio em $z$,

$$
P\left(Y_{1}+z \mu\left(Y_{1}\right), \ldots, Y_{l}+z \mu\left(Y_{l}\right)\right)=P_{\mu}^{(0)}+P_{\mu}^{(1)} z+\cdots P_{\mu}^{(d)} z^{d}
$$

definindo assim elementos $P_{\mu}^{(i)} \in S(\mathfrak{g})$, chamados $\mu$-deslocamentos de P. Denotamos por $\overline{\mathcal{A}}_{\mu}$ a subálgebra de $S(\mathfrak{g})$ gerada pelos $\mu$-deslocamentos de todos polinômios $\mathfrak{g}$-invariantes $P$. A subálgebra $\overline{\mathcal{A}}_{\mu}$ é chamada de subálgebra de Mishchenko-Fomenko.

As subálgebras de Mishchenko-Fomenko são subálgebras maximais Poisson comutativas de $\mathrm{S}(\mathfrak{g})$. O problema de Vinberg proposto em Vinberg [1991] consiste em quantizar as subálgebras de Mishchenko-Fomenko, ou seja, encontrar subálgebras comutativas de $U(L)$ cujas álgebras graduadas são as subálgebras de Mishchenko-Fomenko. Soluções positivas para o problema de Vinberg foram dadas em Rybnikov [2006] (para $\mu$ regular e semissimples) e em Feigin et al. [2010] (para qualquer $\mu$ regular).

A solução de Feigin, Frenkel e Toledano-Laredo usa o centro de Feigin-Frenkel. Em Futorny e Molev [2015], Futorny e Molev exibem geradores das álgebras que quantizam as subálgebras de MishchenkoFomenko em álgebras de Lie do tipo A e também ensinam como escolher os geradores de modo a obter um conjunto de geradores algebricamente independente. Em Molev [2013], Molev exibe geradores para o centro de Feigin-Frenkel para as álgebras de Lie de tipo B, C e D.

Se existir uma álgebra $A_{\mu}$ que quantiza as subálgebras de Mishchenko-Fomenko, podemos nos perguntar se $\mathrm{U}(\mathfrak{g})$ é um $A_{\mu}$-módulo livre. Para isso, estuda-se se os geradores de $\overline{A_{\mu}}$ formam uma sequência regular. 
Para as álgebras de Lie do tipo A, temos as subálgebras de Gelfand-Tsetlin, que também são subálgebras de $U(L)$ comutativas.

Definição 1.0.2. Seja $\mathfrak{g l}_{n}$ a álgebra de Lie linear geral (tipo A), que consiste das matrizes $n \times n$, com base canônica $\left\{E_{i j} \mid 1 \leq i, j \leq n\right\}$. Para $1 \leq m \leq n$, considere $\mathfrak{g l}_{m}$ a álgebra de Lie gerada por $\left\{E_{i j} \mid 1 \leq i, j \leq m\right\}$. Assim temos:

$$
\mathfrak{g l}_{1} \subset \mathfrak{g l}_{2} \subset \ldots \subset \mathfrak{g l}_{n}
$$

$e:$

$$
U\left(\mathfrak{g l}_{1}\right) \subset U\left(\mathfrak{g l}_{2}\right) \subset \ldots \subset U\left(\mathfrak{g l}_{n}\right) .
$$

Denote o centro de $U\left(\mathfrak{g l}_{m}\right)$ por $Z_{m}$ para todo $m \in\{1, \ldots, n\}$. A subálgebra de Gelfand-Tsetlin $\Gamma$ de $U\left(\mathfrak{g l}_{n}\right)$ é definida como a subálgebra gerada por $\left\{Z_{1}, \ldots, Z_{n}\right\}$.

Essas subálgebras são um tipo de limite das subálgebras que quantizam as subálgebras de Mishchenko-Fomenko. Isso nos permite concluir resultados sobre $A_{\mu}$ e $\overline{A_{\mu}}$ a partir de resultados sobre $\Gamma$.

Para o tipo $B$ e $C$, também pode-se estudar o limite das subálgebras que quantizam as subálgebras de Mishchenko-Fomenko. No artigo Molev e Yakimova [2017] são apresentados geradores para uma subálgebra de $\mathfrak{s p}_{2 n}$ (tipo C) que é o análogo das subálgebra de Gelfand-Tsetlin para $\mathfrak{g l}_{n}$. Para aprender mais sobre essas subálgebras, estudamos as variedade de Gelfand-Tsetlin, já que a equidimensionalidade dessas variedades podem implicar que o conjunto gerador das subálgebras de Gelfand-Tsetlin formam uma sequência regular. Nessa dissertação são estudadas especificamente as variedade de Gelfand-Tsetlin de $\mathfrak{s p}_{4}$ e $\mathfrak{s p}_{6}$. São provados, até onde sabemos de forma inédita, os seguintes resultados:

Teorema 1.0.1. A variedade de Gelfand-Tsetlin $\mathfrak{G}_{\mathfrak{s p}_{4}}$ é equidimensional com dimensão 4 .

Teorema 1.0.2. A variedade de Gelfand-Tsetlin $\mathfrak{G}_{\mathfrak{s p}_{6}}$ é equidimensional de dimenão 9 se e somente se a variedade

$$
\mathcal{V}\left(X_{11}, X_{22}, X_{33}, X_{34}, X_{24}, 2 X_{23} X_{32}+X_{25} X_{52}, A, X_{23}^{2}+X_{25} X_{43}, \phi_{6}^{(0)}, \partial_{X_{11}} \phi_{6}^{(0)}, \phi_{4}^{(0)}, \partial_{X_{11}} \phi_{4}^{(0)}\right)
$$

é equidimensional de dimensão 9, onde $A=2 X_{12} X_{21}+2 X_{13} X_{31}+2 X_{14} X_{41}+2 X_{15} X_{51}+X_{16} X_{61}$ $e$ :

$$
\operatorname{det}\left(\begin{array}{cccccc}
u+X_{11} & X_{12} & X_{13} & X_{14} & X_{15} & X_{16} \\
X_{21} & u+X_{22} & X_{23} & X_{24} & X_{25} & X_{15} \\
X_{31} & X_{32} & u+X_{33} & X_{34} & X_{24} & X_{14} \\
X_{41} & X_{42} & X_{43} & u-X_{33} & -X_{23} & -X_{13} \\
X_{51} & X_{52} & X_{42} & -X_{32} & u-X_{22} & -X_{12} \\
X_{61} & X_{51} & X_{41} & -X_{31} & -X_{21} & u-X_{11}
\end{array}\right)=u^{6}+\phi_{2}^{(0)} u^{4}+\phi_{4}^{(0)} u^{2}+\phi_{6}^{(0)}
$$




\section{Capítulo 2}

\section{Conceitos preliminares}

\section{$2.1 \quad$ Álgebra de Lie}

\subsubsection{Conceitos básicos e exemplos}

Definição 2.1.1. Seja $\mathfrak{g}$ um $k$-espaço vetorial com uma operação (produto) $[]:, \mathfrak{g} \times \mathfrak{g} \rightarrow \mathfrak{g}$, $(x, y) \mapsto[x, y]$, chamada de colchete ou comutador. $\mathfrak{g}$, munido dessa operação, é chamado de álgebra de Lie se os seguintes axiomas são satisfeitos:

(i) A operação [, ] é bilinear.

(ii) [, ] é antissimétrico, ou seja, $[x, x]=0$, para todo $x \in \mathfrak{g}$.

(iii) (Identidade de Jacobi) $[x,[y, z]]+[y,[z, x]]+[z,[x, y]]=0$, para todos $x, y, z \in \mathfrak{g}$.

Observação 2.1.1. Se $[$,$] é bilinear e[x, x]=0$, para todo $x \in \mathfrak{g}$, então $[x, y]=-[y, x]$, para todos $x, y \in \mathfrak{g}$, pois:

$$
0=[x+y, x+y]=[x, x]+[y, x]+[x, y]+[y, y]=[y, x]+[x, y] .
$$

Se $k$ não tem característica 2, [, ] é bilinear e $[x, y]=-[y, x]$, para todos $x, y \in \mathfrak{g}$, então $[x, x]=0$, para todo $x \in \mathfrak{g}$, pois:

$$
[x, x]=-[x, x] \Longrightarrow 2[x, x]=0 \Longrightarrow[x, x]=0 .
$$

Normalmente denotaremos uma álgebra de Lie pelo seu espaço vetorial, $\mathfrak{g}$, em vez de denotá-la por $(\mathfrak{g},[]$,$) .$

Definição 2.1.2. Uma álgebra de Lie $\mathfrak{g}$ é dita abeliana se $[x, y]=0$ para todos $x, y \in \mathfrak{g}$.

Definição 2.1.3. Seja $\mathfrak{h} \subset \mathfrak{g}$ um subespaço da álgebra de Lie $\mathfrak{g}$. Chamamos $\mathfrak{h}$ de subálgebra se para todo $x, y \in \mathfrak{h}$ temos $[x, y] \in \mathfrak{h}$.

Abaixo estão listados alguns exemplos de álgebras de Lie:

Exemplo 2.1.1. Seja A uma k-álgebra associativa. Podemos fornecer a A uma estrutura de álgebra de Lie, definindo $[x, y]=x y-y x$ para todos $x, y \in A$.

Exemplo 2.1.2. Seja $V$ um $k$-espaço vetorial e seja End $(V)$ o conjunto das transformaçôes lineares que vão de $V$ em $V$. End(V) com o colchete dado por $[T, S]=T \circ S-S \circ T$, para todos $T, S \in$ End(V), é uma álgebra de Lie chamada álgebra linear geral e é denotada por $\mathfrak{g l}(V)$. Se a dimensão de $V$ é $n, \mathfrak{g l}(V)$ pode ser vista como a álgebra de Lie das matrizes n por $n$, com $[X, Y]=X Y-Y X$, para todas matrizes $X, Y n$ por $n$. Nesse caso, ela será denotada por $\mathfrak{g l}_{n}(k)$.

Exemplo 2.1.3. Álgebra de Lie de tipo $A_{n}$ :

A álgebra linear especial, denotada por $\mathfrak{s l}_{n+1}(k)$, é a subálgebra de $\mathfrak{g l}_{n+1}(k)$ formada pelas matrizes com traço zero. 
Exemplo 2.1.4. Álgebra de Lie de tipo $C_{n}$ :

A álgebra simplética, denotada por $\mathfrak{s p}_{2 n}(k)$, é a subálgebra de $\mathfrak{g l}_{2 n}(k)$ formada pelas matrizes $X$ tais que $J X+X^{t} J=0$, sendo $J=\left(\begin{array}{cc}0 & I d_{n} \\ -I d_{n} & 0\end{array}\right)$, onde 0 é a matriz nula $n$ por $n$ e $I d_{n}$ é a matriz identidade $n$ por $n$.

Exemplo 2.1.5. Álgebra de Lie de tipo $B_{n}$ :

A álgebra ortogonal, denotada por $\mathfrak{a}_{2 n+1}(k)$, é uma subálgebra de $\mathfrak{g l}_{2 n+1}(k)$ formada pelas matrizes $X$ tais que $K X+X^{t} K=0$, sendo $K=\left(\begin{array}{ccc}1 & 0 & 0 \\ 0 & 0 & I d_{n} \\ 0 & I d_{n} & 0\end{array}\right)$, onde 0 é a matriz nula n por $n e$ $I d_{n}$ é a matriz identidade $n$ por $n$.

Exemplo 2.1.6. Álgebra de Lie de tipo $D_{n}$ :

Também chamamos de álgebra ortogonal a álgebra, denotada por $\mathfrak{o}_{2 n}(k)$, que é uma subálgebra de $\mathfrak{g l}_{2 n}(k)$ formada pelas matrizes $X$ tais que $M X+X^{t} M=0$, sendo $M=\left(\begin{array}{cc}0 & I d_{n} \\ I d_{n} & 0\end{array}\right)$, onde 0 é a matriz nula $n$ por $n$ e $I d_{n}$ é a matriz identidade $n$ por $n$.

Exemplo 2.1.7. $\mathfrak{n}(n, \mathbb{K})$ é a subálgebra de $\mathfrak{g l}(n, \mathbb{K})$ formada pelas matrizes triangulares superiores com zeros na diagonal $\left(M=\left(a_{i j}\right)\right.$ com $a_{i j}=0$ se $\left.i \geq j\right)$.

Exemplo 2.1.8. $\mathfrak{t}(n, \mathbb{K})$ é a subálgebra de $\mathfrak{g l}(n, \mathbb{K})$ formada pelas matrizes triangulares superiores $\left(M=\left(a_{i j}\right)\right.$ com $a_{i j}=0$ se $\left.i>j\right)$.

Exemplo 2.1.9. $\mathfrak{d}(n, \mathbb{K})$ é a subálgebra de $\mathfrak{g l}(n, \mathbb{K})$ formada pelas matrizes diagonais.

Definição 2.1.4. Um ideal é um subespaço i de uma álgebra de Lie $\mathfrak{g}$ tal que, se $x \in \mathfrak{g}$ e y $\in \mathfrak{i}$, então $[x, y] \in \mathfrak{i}$.

Exemplo 2.1.10. $\{0\}$ e $\mathfrak{g}$ são ideais de $\mathfrak{g}$.

Exemplo 2.1.11. Chamamos de centro de $\mathfrak{g}$ o conjunto $Z(\mathfrak{g})=\{y \in \mathfrak{g} \mid[x, y]=0, \forall x \in \mathfrak{g}\} . Z(\mathfrak{g})$ é um ideal de $\mathfrak{g}$.

Definição 2.1.5. Uma álgebra de Lie $\mathfrak{g}$ é dita simples se $[\mathfrak{g}, \mathfrak{g}] \neq 0$ e seus únicos ideais são $\{0\}$ e $\mathfrak{g}$.

Para $k$ um corpo algebricamente fechado de característica 0 existem, a menos de isomorfismo, nove famílias de $k$-álgebras de Lie simples de dimensão finita: $A_{n}, B_{n}, C_{n}, D_{n}, E_{6}, E_{7}, E_{8}, F_{4}$ e $G_{2}$. Essas álgebras podem ser representadas por diagramas de Coxeter-Dynkin e por matrizes de Cartan. Para mais informações, consulte o capítulo III (Root Systems), seção 11 (Classification) de Humphreys [1972].

Definição 2.1.6. Um homomorfismo entre álgebras de Lie $\mathfrak{g}, \mathfrak{h}$ é uma transformação linear $\varphi: \mathfrak{g} \rightarrow \mathfrak{h}$ tal que $\varphi([x, y])=[\varphi(x), \varphi(y)]$, para todos $x, y \in \mathfrak{g}$.

\subsubsection{Representação e módulo}

Definição 2.1.7. Uma representação de uma álgebra de Lie $\mathfrak{g}$ é um par $(V, \varphi)$, onde $V$ é um $k$ espaço vetorial e $\varphi: \mathfrak{g} \rightarrow \mathfrak{g l}(V)$ é um homomorfismo de álgebras de Lie. Normalmente denotamos a representação $(V, \varphi)$ só por $\varphi$.

Exemplo 2.1.12. Seja $\mathfrak{g}$ uma álgebra de Lie. Dado $x \in \mathfrak{g}$, a função ad $(x): \mathfrak{g} \rightarrow \mathfrak{g}, y \mapsto[x, y]$ é um homomorfismo. Defina agora a função ad que leva $x \in \mathfrak{g}$ em ad $(x)$. ad é uma representação de $\mathfrak{g}$.

Definição 2.1.8. Sejam g uma álgebra de Lie e $V$ um k-espaço vetorial. Dizemos que $V$ é um $\mathfrak{g}$-módulo se $V$ possui uma operação $\mathfrak{g} \times V \rightarrow V,(x, v) \mapsto x v$ (denotamos o resultado da operação por $x v)$, que satisfaz: 
(i) $(a x+b y) v=a(x v)+b(y v)$, para todos $a, b \in k, x, y \in \mathfrak{g}, v \in V$,

(ii) $x(a v+b u)=a(x v)+b(x u)$, para todos $a, b \in k, x \in \mathfrak{g}, v, u \in V$,

(iii) $[x, y] v=x(y v)-y(x v)$, para todos $x, y \in \mathfrak{g}, v \in V$.

Observação 2.1.2. Representaçôes e módulos são basicamente a mesma coisa:

Seja $\varphi: \mathfrak{g} \rightarrow \mathfrak{g l}(V)$ uma representação. Defina a operação $\mathfrak{g} \times V \rightarrow V$ tal que $(x, v) \mapsto x v=$ $\varphi(x)(v)$, para todos $x \in \mathfrak{g}, v \in V . V$ é um $\mathfrak{g}$-módulo com essa operação.

Por outro lado, seja $V$ um $\mathfrak{g}$-módulo. Defina a função $\varphi: \mathfrak{g} \rightarrow \mathfrak{g l}(V)$ tal que $\varphi(x) \in \mathfrak{g l}(V)$ é a transformação linear que leva $v \in V$ em $x v . \varphi$ é uma representação de $\mathfrak{g}$.

\subsection{3 Álgebra de Lie semissimples}

Sejam $\mathfrak{h}, \mathfrak{l} \subset \mathfrak{g}$ subálgebras. Indicamos por $[\mathfrak{h}, \mathfrak{g}]$ a álgebra de Lie gerada pelo conjunto $\{[x, y] \mid x \in \mathfrak{h}, y \in \mathfrak{l}\}$.

Definição 2.1.9. Defina a seguinte sequência de ideais:

$$
\begin{aligned}
\mathfrak{g}^{(0)} & =\mathfrak{g} \\
\mathfrak{g}^{(1)} & =[\mathfrak{g}, \mathfrak{g}] \\
\mathfrak{g}^{(2)} & =\left[\mathfrak{g}^{(1)}, \mathfrak{g}^{(1)}\right] \\
\vdots & \\
\mathfrak{g}^{(k)} & =\left[\mathfrak{g}^{(k-1)}, \mathfrak{g}^{(k-1)}\right]
\end{aligned}
$$

Essa sequência de ideais é a série derivada de $\mathfrak{g}$.

Definição 2.1.10. Uma álgebra $\mathfrak{g}$ é dita solúvel se sua série derivada se anula em algum momento, ou seja, se existe $n \in \mathbb{N}$ tal que $\mathfrak{g}^{(n)}=0$.

Definição 2.1.11. Uma álgebra de Lie é semissimples se o único ideal solúvel que ela contém é o $\{0\}$.

Exemplo 2.1.13. Toda álgebra de Lie simples é também semissimples.

Definição 2.1.12. Seja $\mathfrak{g}$ uma álgebra de Lie de dimensão finita. Para uma base de $\mathfrak{g}$, podemos escrever adx em forma de matriz, para todo $x \in \mathfrak{g}$. Defina $\kappa(x, y)=\operatorname{tr}($ adx ady $) . \kappa$ é chamada de forma de Killing.

A forma de Killing é:

- bilinear,

- é simétirca, ou seja, $\kappa(x, y)=\kappa(y, x)$ para todo $x, y \in \mathfrak{g}$,

- é associativa da seguinte forma $\kappa([x, y], z)=\kappa(x,[y, z])$.

Definição 2.1.13. Uma forma bilinear simétrica $\beta$ é dita não degenerada se seu radical $S=\{x \in \mathfrak{g} \mid \beta(x, y)=0, \forall y \in \mathfrak{g}\}$ é $\{0\}$.

Para uma álgebra $\mathfrak{g}$ com dimensão $n$ e base $\left\{v_{1}, \ldots, v_{n}\right\}$, uma forma bilinear $\beta$ pode ser expressa pela matriz $M=\left(a_{i j}\right)$, onde $a_{i j}=\beta\left(v_{i}, v_{j}\right)$, para todo $i, j \in\{1, \ldots, n\}$ e temos $\beta(x, y)=x^{T} M y$ para todos $x, y \in \mathfrak{g}$. A forma $\beta$ é não degenerada se e somente se o determinante da sua matriz for diferente de 0 .

Teorema 2.1.1. Seja $\mathfrak{g}$ uma álgebra de Lie. Então $\mathfrak{g}$ é semissimples se e somente se sua forma de Killing for não degenerada. 
Teorema 2.1.2. Seja $\mathfrak{g}$ uma álgebra de Lie semissimples. Então existem ideais de $\mathfrak{g}$ simples $\mathfrak{i}_{1}, \ldots, \mathfrak{i}_{t}$ tais que $\mathfrak{g}=\mathfrak{i}_{1} \oplus \ldots \oplus \mathfrak{i}_{t}$. Todo ideal simples de $\mathfrak{g}$ coincide com algum $\mathfrak{i}_{i}$. Além disso, a forma de Killing em $\mathfrak{i}_{i}$ é a restrição de $\kappa$ em $\mathfrak{i}_{i} \times \mathfrak{i}_{i}$.

Demonstração. Ver página 23 de Humphreys [1972].

\section{2 Álgebra graduada e álgebra filtrada}

Definição 2.2.1. Uma álgebra A é uma álgebra graduada se existe uma coleção de subespaços vetoriais $\left\{A_{n}\right\}_{n \in \mathbb{N}}$ (chamada de graduação de A), tal que:

(i) $A_{n} A_{m} \subset A_{n+m}$, para todos $n, m \in \mathbb{N}$,

(ii) $A=\bigoplus_{n=0}^{\infty} A_{n}$.

Definição 2.2.2. Sejam $A$ e $B$ duas álgebras graduadas com graduações $\left\{A_{n}\right\}_{n \in \mathbb{N}} e\left\{B_{n}\right\}_{n \in \mathbb{N}}$, respectivamente. Um homomorfismo de álgebras graduadas $f: A \rightarrow B$ é um homomorfismo de álgebras tal que $f\left(A_{n}\right) \subset B_{n}$ para todo $n \in \mathbb{N}$.

Definição 2.2.3. Uma álgebra A é uma álgebra filtrada se existe uma coleção de subespaços vetoriais $\left\{A_{n}\right\}_{n \in \mathbb{N}}$ (chamada de filtração de A), tal que:

(i) $A_{0} \subset A_{1} \subset A_{2} \subset \ldots$ (ou seja, se $n \leq m$, então $A_{n} \subset A_{m}$ ),

(ii) $A_{n} A_{m} \subset A_{n+m}$, para todos $n, m \in \mathbb{N}$,

(iii) $A=\bigcup_{n=0}^{\infty} A_{n}$.

Seja $A$ uma álgebra filtrada com filtração $\left\{A_{n}\right\}_{n \in \mathbb{N}}$. Defina $\operatorname{gr}(A)=\bigoplus_{n=0}^{\infty} \overline{A_{n}}$, onde $A_{-1}:=\{0\}$ e $\overline{A_{n}}:=A_{n} / A_{n-1}$ para todo $n \in \mathbb{N}$. Vamos definir três operações em $\operatorname{gr}(A)$ :

- Soma:

Sejam $x+A_{n-1}, y+A_{m-1} \in \operatorname{gr}(A)$ com $n \leq m$. Como $A_{n} \subset A_{m}$ e $A_{m}$ é subespaço, temos que $x+y \in A_{m}$. Assim podemos definir $\left(x+A_{n-1}\right)+\left(y+A_{m-1}\right):=x+y+A_{m-1}$.

- Multiplicação por escalar:

Sejam $a \in k$ e $x+A_{n-1} \in \operatorname{gr}(A)$. Como $A_{n}$ é subespaço, ax $\in A_{n}$. Assim podemos definir $a\left(x+A_{n-1}\right):=a x+A_{n-1}$.

- Multiplicação:

Sejam $x+A_{n-1}, y+A_{m-1} \in \operatorname{gr}(A)$. Como $A_{n} A_{m} \subset A_{n+m}$, temos que $x y \in A_{n+m}$. Então podemos definir $\left(x+A_{n-1}\right)\left(y+A_{m-1}\right):=x y+A_{m+n-1}$.

Todas operações estão bem definidas e com elas $\operatorname{gr}(A)$ é uma álgebra graduada com graduação associada a filtração de A.

Considere agora $B$ uma subálgebra de $A$. Então $B$ também é uma álgebra filtrada, cuja filtração induzida é $\left\{B_{n}\right\}_{n \in \mathbb{N}}$ onde $B_{n}=A_{n} \cap B$ para todo $n \in \mathbb{N}$. Assim, podemos definir a álgebra graduada $\operatorname{gr}(B)$. A inclusão $i: B \rightarrow A$ induz um morfismo de álgebras graduadas:

$$
\begin{aligned}
\operatorname{gr}(i): \operatorname{gr}(B) & \rightarrow \operatorname{gr}(A) \\
x+B_{n-1} & \mapsto x+A_{n-1}
\end{aligned}
$$

$g r(i)$ é injetor, pois se $x+B_{n-1}, y+B_{n-1} \in \operatorname{gr}(B)$ são tais que $\operatorname{gr}(i)\left(x+B_{n-1}\right)=\operatorname{gr}(i)\left(y+B_{n-1}\right)$, então $x+A_{n-1}=y+A_{n-1}$. Isto implica que $x-y \in A_{n-1}$. Assim $x-y \in A_{n-1} \cap B=B_{n-1} \mathrm{e}$ concluimos que $x+B_{n-1}=y+B_{n-1}$. Portanto, podemos identificar $\operatorname{gr}(B)$ com a subálgebra de $\operatorname{gr}(A)$ cujos elementos da forma $x+A_{n-1}$ tem $x \in B_{n}$. 


\section{$2.3 \quad$ Álgebra tensorial}

Seja $R$ um anel e $M$ um $R$-módulo. Considere os seguintes $R$-módulos:

$$
\begin{aligned}
& T^{0} M=R \\
& T^{1} M=M \\
& T^{i} M=M \otimes_{R} \cdots \otimes_{R} M \quad \text { (i vezes), para todo inteiro } i>1 .
\end{aligned}
$$

e:

$$
T(M)=\bigoplus_{i=0}^{\infty} T^{i} M .
$$

Podemos munir $T(M)$ de um produto associativo, definindo o produto nos geradores homogêneos de $T(M)$ da seguinte maneira:

se $v_{1} \otimes \cdots \otimes v_{k} \in T^{k} M, w_{1} \otimes \cdots \otimes w_{m} \in T^{m} M$ :

$$
\left(v_{1} \otimes \cdots \otimes v_{k}\right)\left(w_{1} \otimes \cdots \otimes w_{m}\right)=v_{1} \otimes \cdots \otimes v_{k} \otimes w_{1} \otimes \cdots \otimes w_{m} \in T^{k+m} M
$$

e estendendo por $R$-bilinearidade para todo $T(M)$. $T(M)$ é chamada de álgebra tensorial de $M$. $T(M)$ é uma álgebra associativa graduada com unidade.

Proposição 2.3.1. (Propriedade Universal da Álgebra Tensorial)

Sejam $M$ um R-módulo e A uma R-álgebra associativa com unidade 1. Para qualquer homomorfismo de $R$-módulos $\varphi: M \rightarrow A$, existe um único homomorfismo de $R$-álgebras $\psi: T(M) \rightarrow A$ tal que, $\psi(1)=1$ e $\psi \circ i=\varphi$, onde $i: M \rightarrow T(M)$ é a inclusão. Em outras palavras, o seguinte diagrama comuta:

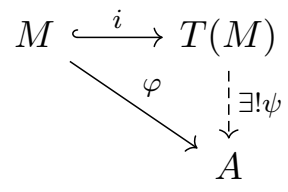

Demonstração. Defina $\psi$ nos geradores homogêneos de $T(M)$ da seguinte maneira:

$$
\psi\left(v_{1} \otimes \cdots \otimes v_{k}\right)=\varphi\left(v_{1}\right) \cdot \ldots \cdot \varphi\left(v_{k}\right)
$$

e estenda por $R$-linearidade para todos elementos de $T(M)$.

$\psi$ está bem definida e é um homomorfismo de $R$-álgebras. Também é fácil ver pela definição que $\psi \circ i=\varphi$.

Proposição 2.3.2. Sejam $M$ um R-módulo livre e $\left\{e_{1}, \ldots, e_{n}\right\}$ uma base de $M$. Então, para qualquer $m \geq 1, T^{m} M$ é um $R$-módulo livre com posto $n^{m}$ e com base:

$$
\left\{e_{i_{1}} \otimes \cdots \otimes e_{i_{m}} \mid 1 \leq i_{1}, \ldots, i_{m} \leq n\right\}
$$

Proposição 2.3.3. Seja $M$ um R-módulo livre de posto $n$ sobre $R$. Então $T(M)$ é isomórfica a álgebra de polinômios não comutativos em n variáveis sobre $R$.

Demonstração. Veja proposição 7.1 de Lang [2002].

\subsection{1 Álgebra simétrica}

Seja $M$ um $R$-módulo e $T(M)$ sua álgebra tensorial. Denote por $C(M)$ o ideal (bilateral) de $T(M)$ gerado pelos elementos da forma $x \otimes y-y \otimes x$, para todo $x, y \in M$.

Definição 2.3.1. A álgebra simétrica de $M$ é a álgebra quociente $T(M) / C(M)$ e a denotamos por $S(M)$. 
Observação 2.3.1. Seja $k$ um corpo de característica zero e seja $M$ um k-espaço vetorial. Outra forma de definir $S(M)$ é considerar $S^{n} M$ a imagem da função

$S^{n}: T^{n} M \rightarrow T^{n} M$ que leva $e_{i_{1}} \otimes \cdots \otimes e_{i_{n}}$ da base de $T^{n} M$ em $\frac{1}{n !} \sum_{\sigma \in S_{n}} e_{i_{\sigma(1)}} \otimes \cdots \otimes e_{i_{\sigma(n)}}\left(S^{n} M e^{\prime}\right.$ o conjunto de elementos de $T^{n} M$ que são simétricos). Depois defina $S(M)=\bigoplus_{n=0}^{\infty} S^{n} M(S(M)$ é o conjunto dos vetores de $T(M)$ que são simétricos).

Para uma breve discussão sobre isso, ver o começo do capitulo 6 (Existence theorem) de Humphreys [1972] e, para mais detalhes, ver capitulo 4 (Multilinear algebra), seção 5 (Symmetric Tensors) de Kostrikin e Manin [1989].

$S(M)$ é uma $R$-álgebra comutativa graduada com graduação $\left\{S^{n} M\right\}_{n \in \mathbb{N}}$, onde $S^{n} M=T^{n}(M) /\left(T^{n}(M) \cap C(M)\right)$ para todo $n \in \mathbb{N}$.

Proposição 2.3.4. Seja $M$ um R-módulo livre com posto $n$ sobre $R$. Então $S(M)$ é isomórfica a álgebra de polinômios em $n$ variáveis sobre $R$.

Demonstração. Ver proposição 8.1 de Lang [2002].

\subsection{2 Álgebra exterior}

Seja $M$ um $R$-módulo e $T(M)$ sua álgebra tensorial. Denote por $A(M)$ o ideal (bilateral) de $T(M)$ gerado pelos elementos da forma $x \otimes x$, para todo $x \in M$.

Definição 2.3.2. A álgebra exterior de $M$ é a álgebra quociente $T(M) / A(M)$ e a denotamos por $\bigwedge M$.

Observação 2.3.2. Seja $k$ corpo de característica zero e seja $M$ um k-espaço vetorial. Outra forma de definir $\bigwedge M$ é considerar $\bigwedge^{n} M$ a imagem da função

$\varphi^{n}: T^{n} M \rightarrow T^{n} M$ que leva $e_{i_{1}} \otimes \cdots \otimes e_{i_{n}}$ da base de $T^{n} M$ em $\frac{1}{n !} \sum_{\sigma \in S_{n}}(\operatorname{sgn\sigma }) e_{i_{\sigma(1)}} \otimes \cdots \otimes e_{i_{\sigma(n)}}$.

Depois defina $\bigwedge M=\bigoplus_{n=0}^{\infty} \bigwedge^{n} M$.

Para mais detalhes, ver capitulo 4 (Multilinear algebra) e seção 6 (Skew-symmetric tensors and the exterior algebra of a linear space) de Kostrikin e Manin [1989].

O produto em $\bigwedge M$ é denotado por $\wedge$ e a classe de $x_{1} \otimes \cdots \otimes x_{k} \in T(M)$ em $\wedge M$ é denotada por $x_{1} \wedge \cdots \wedge x_{k}$.

$\bigwedge M$ é uma álgebra graduada com graduação $\left\{\bigwedge^{n} M\right\}_{n \in \mathbb{N}}$ onde $\bigwedge^{n} M=T^{n} M /\left(T^{n} M \cap A(M)\right)$ para todo $n \in \mathbb{N}$. $\bigwedge^{n} M$ é chamada de n-ésima potência exterior de $M$.

Proposição 2.3.5. (Propriedade universal da n-ésima potência exterior de $M$ )

Seja $n \in \mathbb{N}$. Se existir um R-módulo $N$ e uma aplicação n-linear alternada $f: M^{n} \rightarrow N$ (isto é, $\left.f\left(x_{1}, x_{2}, \ldots, x, \ldots, x, \ldots, x_{n}\right)=0\right)$, então existe um único homomorfismo de $R$-módulos

$g: \bigwedge^{n} M \rightarrow N$ tal que $g \circ \wedge_{n}=f$, onde $\wedge_{n}: M^{n} \rightarrow \bigwedge^{n} M$ é a função que leva $\left(m_{1}, \ldots, m_{n}\right)$ em $m_{1} \wedge \cdots \wedge m_{n}$, para todo $\left(m_{1}, \ldots, m_{n}\right) \in M^{n}$. Em outras palavras, o seguinte diagrama comuta:

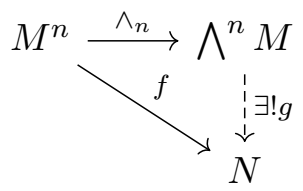

Demonstração. Ver proposição 7 do parágrafo 7 do capítulo 3 de Bourbaki [1998].

Na proposição a seguir são apresentadas algumas características da álgebra exterior e de suas $n$-ésimas potências. 
Proposição 2.3.6. (i) Seja $M$ um R-módulo. Sejam $x_{1}, \ldots, x_{k} \in M$. Para todo $\sigma \in S_{k}$, temos:

$$
x_{\sigma(1)} \wedge \cdots \wedge x_{\sigma(k)}=(\operatorname{sgn} \sigma) x_{1} \wedge \cdots \wedge x_{k}
$$

onde sgn é o sinal da permutação $\sigma$.

(ii) Seja $M$ um R-módulo de posto n e base $\left\{e_{1}, \ldots, e_{n}\right\}$, então, para cada $1 \leq k \leq n, \bigwedge^{k} M$ é um $R$-módulo livre com base $\left\{e_{i_{1}}, \ldots, e_{i_{k}} \mid 1 \leq i_{1}, \ldots, i_{k}, \leq n\right\}$ e tem posto $\left(\begin{array}{c}n \\ k\end{array}\right)$. Se $k>n$, temos $\bigwedge^{k} M=0$.

(iii) Seja $M$ um R-módulo de posto $n$. Então $\bigwedge M$ é um $R$-módulo livre de posto $2^{n}$.

Demonstração. (i) Ver página 510 de Bourbaki [1998].

(ii) e (iii) Ver página 281 de Kostrikin e Manin [1989].

\subsection{3 Álgebra envolvente universal}

Definição 2.3.3. Seja $\mathfrak{g}$ uma álgebra de Lie sobre $k$. A álgebra envolvente universal de $\mathfrak{g}$ é um par $(U, i)$, onde $U$ é uma k-álgebra associativa com unidade 1 e $i: \mathfrak{g} \rightarrow U$ é uma transformação linear que satisfaz:

(i) $i([x, y])=i(x) i(y)-i(y) i(x)$, para todo $x, y \in \mathfrak{g}$,

(ii) (propriedade universal) para qualquer $k$-álgebra associativa $A$ com 1 e para qualquer transformação linear $j: \mathfrak{g} \rightarrow A$ que satisfaz (i), existe um único homomorfismo de k-álgebras associativas $\varphi: U \rightarrow A$, que manda unidade em unidade, tal que $\varphi \circ i=j$. Ou seja, o diagrama abaixo comuta:

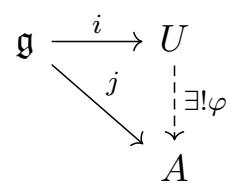

Proposição 2.3.7. Dada uma álgebra de Lie $\mathfrak{g}$, existe uma álgebra envolvente universal para $\mathfrak{g}$.

Demonstração. Seja $T(\mathfrak{g})$ a álgebra tensorial de $\mathfrak{g}$ e seja $J$ o ideal bilateral gerado pelos elementos $x \otimes y-y \otimes x-[x, y]$, para todos $x, y \in \mathfrak{g}$. Defina $U(\mathfrak{g})=T(\mathfrak{g}) / J$ e considere $\pi: T(\mathfrak{g}) \rightarrow U(\mathfrak{g})$ o homomorfismo sobrejetor canônico. Denote por $i$ a restrição de $\pi$ a $\mathfrak{g}$. Vamos mostrar que $(U(\mathfrak{g}), i)$ é uma álgebra envolvente universal para $\mathfrak{g}$.

(i)

$$
\begin{aligned}
i([x, y]) & =\pi([x, y])=[x, y]+J=x \otimes y-y \otimes x+J=(x+J)(y+J)-(y+J)(x+J)= \\
& =i(x) i(y)-i(y) i(x)
\end{aligned}
$$

para todos $x, y \in \mathfrak{g}$

(ii) Seja $A$ uma $k$-álgebra associativa com 1 e $j: \mathfrak{g} \rightarrow A$ uma transformação linear tal que $j([x, y])=j(x) j(y)-j(y) j(x)$, para todos $x, y \in \mathfrak{g}$. Pela propriedade universal da álgebra tensorial, existe um único homomorfismo de $k$-álgebras $\psi: T(\mathfrak{g}) \rightarrow A$ tal que $\psi(1)=1$ e $\psi \circ e=j$, onde $e: \mathfrak{g} \rightarrow T(\mathfrak{g})$ é a inclusão. $J \subset \operatorname{ker} \psi$, pois:

$$
\begin{aligned}
\psi(x \otimes y-y \otimes x-[x, y]) & =\psi \circ e(x) \psi \circ e(y)-\psi \circ e(y) \psi \circ e(x)-\psi \circ e([x, y])= \\
& =j(x) j(y)-j(y) j(x)-j([x, y])=0
\end{aligned}
$$

para todos $x, y \in \mathfrak{g}$. Pelo teorema do homomorfismo para quocientes, existe $\varphi: T(\mathfrak{g}) / J \rightarrow A$ tal que $\varphi \circ \pi=\psi$. Assim, temos: $\varphi \circ i=\varphi \circ \pi \circ e=\psi \circ e=j$ e $\varphi(1)=1$. 
E $\varphi$ é o único homomorfismo que satisfaz isso, pois, se $\varphi^{\prime}$ também satisfaz isso, temos:

$$
\begin{aligned}
\varphi^{\prime}\left(x_{1} \otimes \cdots \otimes x_{m}+J\right) & =\varphi^{\prime}\left(\pi\left(x_{1} \otimes \cdots \otimes x_{m}\right)\right)=\varphi^{\prime}\left(i\left(x_{1}\right) \cdots i\left(x_{m}\right)\right)=\varphi^{\prime}\left(i\left(x_{1}\right)\right) \cdots \varphi^{\prime}\left(i\left(x_{m}\right)\right)= \\
& =j\left(x_{1}\right) \cdots j\left(x_{m}\right)=\varphi \circ i\left(x_{1}\right) \cdots \varphi \circ i\left(x_{m}\right)=\varphi\left(x_{1} \otimes \cdots \otimes x_{m}+J\right)
\end{aligned}
$$

para todo $x_{1} \otimes \cdots \otimes x_{m}+J \in T(\mathfrak{g}) / J$.

Portanto, $(U(\mathfrak{g}), i)$ é uma álgebra envolvente universal para $\mathfrak{g}$.

Proposição 2.3.8. Dada uma álgebra de Lie $\mathfrak{g}$, existe, a menos de isomorfismo, uma única álgebra envolvente universal. Ela será denotada por $U(\mathfrak{g})$.

Demonstração. Sejam $\left(U_{1}, i_{1}\right)$ e $\left(U_{2}, i_{2}\right)$ álgebras envolventes universais de $\mathfrak{g}$. Então, existe um único homomorfismo de álgebras $\varphi_{1}: U_{1} \rightarrow U_{2}$ tal que $\varphi_{1}(1)=1$ e $\varphi_{1} \circ i_{1}=i_{2}$.

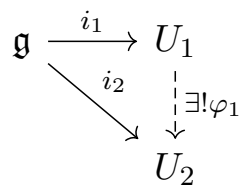

Analogamente, existe um único homomorfismo de álgebras $\varphi_{2}: U_{2} \rightarrow U_{1}$ tal que $\varphi_{2}(1)=1 \mathrm{e}$ $\varphi_{2} \circ i_{2}=i_{1}$.

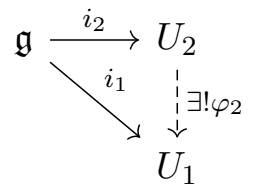

Assim, temos:

$$
\varphi_{2} \circ \varphi_{1} \circ i_{1}=\varphi_{2} \circ i_{2}=i_{1}=I d_{U_{1}} \circ i_{1}
$$

onde $I d_{U_{1}}$ é o homomorfismo identidade de $U_{1}$.

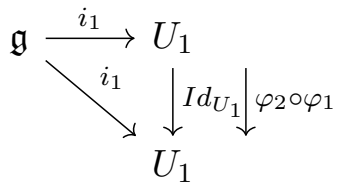

Como $\left(U_{1}, i_{1}\right)$ é uma álgebra envolvente universal para $\mathfrak{g}$, pela propriedade universal concluimos que $\varphi_{2} \circ \varphi_{1}=I d_{U_{1}}$. Analogamente, temos $\varphi_{1} \circ \varphi_{2}=I d_{U_{2}}$. Portanto, $\varphi_{1}$ e $\varphi_{2}$ são isomorfismos e $U_{1} \cong U_{2}$.

Exemplo 2.3.1. Seja $\mathfrak{g}$ uma álgebra de Lie abeliana. Então o ideal bilateral J gerado pelos elementos da forma $x \otimes y-y \otimes x-[x, y]$, para todos $x, y \in \mathfrak{g}$, é igual ao ideal $C(\mathfrak{g})$. Assim temos: $U(\mathfrak{g})=T(\mathfrak{g}) / J=T(\mathfrak{g}) / C(\mathfrak{g})=S(\mathfrak{g})$. Ou seja, a álgebra envolvente universal de $\mathfrak{g}$ é a álgebra simétrica de $\mathfrak{g}$.

Seja $\mathfrak{g}$ uma álgebra de Lie. Simplificaremos as notações da seguinte maneira:

$$
U:=U(\mathfrak{g}), \quad T:=T(\mathfrak{g}), \quad T^{m}:=T^{m}(\mathfrak{g}), \quad S:=S(\mathfrak{g}), \quad S^{m}:=S^{m}(\mathfrak{g}), \quad C:=C(\mathfrak{g})
$$

Considere a seguinte filtração de $T:\left\{T_{m}\right\}_{m \in \mathbb{N}}$, onde $T_{m}=\bigoplus_{i=0}^{m} T^{m}$, para todo $m \in \mathbb{N}$. Podemos definir em $U$ uma filtração $\left\{U_{m}\right\}_{m \in \mathbb{N}}$ onde $U_{m}=\pi\left(T_{m}\right)$, para todo $m \in \mathbb{N}$. Seja $\pi: T \rightarrow U$ 
a projeção canônica. Como temos uma filtração, podemos considerar a álgebra graduada de $U$, $\operatorname{gr}(U)=\bigoplus_{m=0}^{\infty} G^{m}$, onde $U_{-1}=\{0\}$ e $G^{m}=U_{m} / U_{m-1}$, para todo $m \in \mathbb{N}$.

Defina a transformação linear $\phi_{m}: T^{m} \rightarrow G^{m}$ que leva $x_{1} \otimes \cdots \otimes x_{m} \in T^{m}$ em $\pi\left(x_{1} \otimes \cdots \otimes x_{m}\right)+U_{m-1}$, para todo $m \in \mathbb{N}$. $\phi_{m}$ é sobrejetora pois $\pi\left(T^{m}-T^{m-1}\right)=U_{m}-U_{m-1}$. Como $T=\underset{m=0}{\infty} T^{m}$, podemos com as transformações lineares $\phi_{m}$ definir uma função linear $\phi: T \rightarrow \operatorname{gr}(U)$. Como $\phi_{m}$ é sobrejetora para todo $m \in \mathbb{N}, \phi$ também é sobrejetora. Note também que $\phi$ manda 1 em 1.

Lema 2.3.1. A função $\phi$ é um homomorfismo de álgebras. Além disso, $\phi(C)=0$, nos permitindo definir um homomorfismo $\omega: S=T / C \rightarrow \operatorname{gr}(U)$ tal que $\omega(x+C)=\phi(x)$, para todo $x \in T$.

Demonstração. Ver página 91 de Humphreys [1972].

Teorema 2.3.1. (Teorema de Poincaré-Birkhoff-Witt ou Teorema PBW)

$O$ homomorfismo $\omega: S \rightarrow g r(U)$ é um isomorfismo de álgebras.

Demonstração. Ver página 93 e 94 de Humphreys [1972].

Corolário 2.3.1. $\quad$ (i) A função canônica $i: \mathfrak{g} \rightarrow U(\mathfrak{g})$ é injetora.

(ii) Seja $\left\{x_{i}\right\}_{i \in I}(I \subset \mathbb{N})$ uma base de $\mathfrak{g}$. Então:

$$
\left\{\pi\left(x_{i(1)} \otimes \cdots \otimes x_{i(m)}\right) \mid i(1), i(2), \ldots, i(m) \in I, i(1) \leq i(2) \leq \ldots \leq i(m)\right\} \cup\{1\}
$$

é uma base para $U(\mathfrak{g})$.

(iii) Seja $\mathfrak{h}$ uma subálgebra de $\mathfrak{g}$. Estenda uma base $\left\{h_{i}\right\}_{i \in I}(I \subset \mathbb{N})$ de $\mathfrak{h}$ para uma base $\left\{h_{i}\right\}_{i \in I} \cup\left\{x_{i}\right\}_{i \in J}(J \subset \mathbb{N})$ de $\mathfrak{g}$. O homomorfismo, que vai de $U(\mathfrak{h})$ em $U(\mathfrak{g})$ e é induzido pela injeção $\mathfrak{h} \rightarrow \mathfrak{g} \rightarrow U(\mathfrak{g})$, é injetor e $U(\mathfrak{g})$ é um $U(\mathfrak{h})$-módulo livremente gerado com base:

$$
\left\{\pi\left(x_{i(1)} \otimes \cdots \otimes x_{i(m)}\right) \mid i(1), i(2), \ldots, i(m) \in J, i(1) \leq i(2) \leq \ldots \leq i(m)\right\} \cup\{1\}
$$

Demonstração. Ver página 92 de Humphreys [1972].

Essa parte sobre o teorema PBW também pode ser vista na primeira seção do capítulo 2 (Enveloping algebras) de Dixmier [1977].

\section{4 Álgebra de Poisson-Lie}

Definição 2.4.1. Seja A uma k-álgebra com produto *. Uma derivação de A é uma transformação linear $d: A \rightarrow A$ que satisfaz a identidade de Leibniz:

$$
d(x * y)=x * d(y)+d(x) * y \quad, \quad \forall x, y \in A .
$$

Definição 2.4.2. Uma álgebra de Poisson-Lie (ou só Poisson) é um k-espaço vetorial equipado com dois produtos bilineares $* e\{\},,(A, *,\{\}$,$) , que satisfazem as seguintes propriedades:$

(i) A com o produto * é uma k-álgebra associativa,

(ii) A com o produto $\{$,$\} é uma k-álgebra de Lie (chamamos \{$,$\} de colchete de Poisson),$

(iii) Dado $x \in A$, a função $\{x\}:, A \rightarrow A$ definida como $\{x\},(y)=\{x, y\}$, para todo $y \in A$, é uma derivação em $(A, *)$. Ou seja, $\{x$,$\} é uma transformação linear e:$

$$
\{x, y * z\}=y *\{x, z\}+\{x, y\} * z
$$

para todos $x, y, z \in A$. 
Definição 2.4.3. Sejam $\left(A, *_{A},\{,\}_{A}\right) e\left(B, *_{B},\{,\}_{B}\right)$ duas álgebras de Poisson-Lie. Uma função $\varphi: A \rightarrow B$ é um homomorfismo de álgebras de Poisson se $\varphi$ é um homormorfismo de álgebras de $\left(A, *_{A}\right)$ em $\left(B, *_{B}\right)$ e é um homormorfismo de álgebras de Lie de $(A,\{\} A$,$) em \left(B,\{,\}_{B}\right)$.

\section{5 Álgebra homológica}

Definição 2.5.1. Seja $R$ um anel. Uma sequência $\left(M_{i}, d_{i}\right)_{i \in \mathbb{Z}}$, onde $M_{i}$ são $R$-módulos e $d_{i}: M_{i} \rightarrow M_{i-1}$ homomorfismos de $R$-módulos, tais que $d_{i} d_{i+1}=0$ para todo $i \in \mathbb{Z}$, é chamada de complexo. Um complexo é denotado por $(M, d)$ e é representado da seguinte forma:

$$
\cdots \longrightarrow M_{i+1} \stackrel{d_{i+1}}{\longrightarrow} M_{i} \stackrel{d_{i}}{\longrightarrow} M_{i-1} \longrightarrow \cdots
$$

Os homomorfismos $d_{i}$ são chamados de diferenciais.

Observação 2.5.1. Algumas vezes são usados complexos indexados por uma sequência crescente de inteiros, ou seja, $d_{i}$ vai de $M_{i}$ em $M_{i+1}, d_{i} d_{i-1}=0$ e temos:

$$
\cdots \longrightarrow M_{i-1} \stackrel{d_{i-1}}{\longrightarrow} M_{i} \stackrel{d_{i}}{\longrightarrow} M_{i+1} \longrightarrow \cdots
$$

Definição 2.5.2. Seja $(M, d)$ um complexo. Para cada $i \in \mathbb{Z}$ definimos sua i-ésima homologia (ou i-ésimo grupo de homologia) como:

$$
H_{i}(M)=Z_{i}(M) / B_{i}(M)
$$

onde $Z_{i}=$ kerd $_{i}$ e $B_{i}=\operatorname{Imd}_{i+1}$.

\subsubsection{Complexo de Koszul}

Seja $R$ um anel, $M$ um $R$-módulo e $f: M \rightarrow R$ uma função $R$-linear. Considere a seguinte função $n$-linear alternada:

$$
\begin{aligned}
M^{n} & \rightarrow \bigwedge^{n-1} M \\
\left(x_{1}, \ldots, x_{n}\right) & \mapsto \sum_{i=1}^{n}(-1)^{i+1} f\left(x_{i}\right) x_{1} \wedge \cdots \wedge \widehat{x}_{i} \wedge \cdots \wedge x_{n}
\end{aligned}
$$

onde $\widehat{x_{i}}$ significa que $x_{i}$ não está nesse produto exterior. Pela propriedade universal da $n$-ésima potência exterior, existe uma função $R$-linear $d_{f}^{n}: \bigwedge^{n} M \rightarrow \bigwedge^{n-1} M$ tal que:

$$
d_{f}^{n}\left(x_{1} \wedge \cdots \wedge x_{n}\right)=\sum_{i=1}^{n}(-1)^{i+1} f\left(x_{i}\right) x_{1} \wedge \cdots \wedge \widehat{x}_{i} \wedge \cdots \wedge x_{n}
$$

para todo $x_{1}, \ldots, x_{n} \in M$. A coleção das funções $\left\{d_{f}^{n}\right\}_{n>0}$ define um $R$-homomorfismo $d_{f}: \bigwedge M \rightarrow$ $\bigwedge M$ cuja restrição a $\bigwedge^{n} M$ é igual a $d_{f}^{n}$.

Proposição 2.5.1. (i) $d_{f} \circ d_{f}=0$.

(ii) Para todo $x, y \in \bigwedge M$ homogêneo: $d_{f}(x \wedge y)=d_{f}(x) \wedge y+(-1)^{\operatorname{deg} x} x \wedge d_{f}(y)$, sendo $x \in \bigwedge^{\operatorname{deg} x} M$.

Demonstração. Os dois itens seguem de cálculos diretos a partir da definição de $d_{f}$.

O item (ii) da proposição anterior mostra que $d_{f}$ é uma antiderivação (de grau -1) e o item (i) prova que a cadeia abaixo é um complexo de $R$-módulos.

$$
\cdots \longrightarrow \bigwedge^{n} M \stackrel{d_{f}}{\longrightarrow} \bigwedge^{n-1} M \longrightarrow \cdots \longrightarrow \bigwedge^{2} M \stackrel{d_{f}}{\longrightarrow} M \stackrel{f}{\longrightarrow} R \longrightarrow 0
$$


Definição 2.5.3. O complexo $\left(\bigwedge M, d_{f}\right)$ acima é chamado de complexo de Koszul de $f$ e é denotado por K•(f). A homologia do complexo de Koszul é chamada de homologia de Koszul de fe é denotada por $H_{\bullet}(f)$.

Definição 2.5.4. Seja $N$ um R-módulo. Chamamos o complexo $\left(\bigwedge M \otimes N, d_{f} \otimes 1\right)$ de complexo de Koszul de $f$ com coeficientes em $N$, denotamos por $K \bullet(f, N)$ e seus diferenciais por $d_{f, N}$. A homologia desse complexo é chamada de homologia de Koszul de $f$ com coeficientes em $N$ e é denotada por $H_{\bullet}(f, N)$.

Seja $M$ um $R$-módulo livre de base $\left\{e_{1}, \ldots, e_{n}\right\}$. Uma função $R$-linear $f: M \rightarrow R$ está unicamente determinada pelos seus valores $x_{i}=f\left(e_{i}\right)$ para todo $i \in\{1, \ldots, n\}$. Da mesma forma, para quaisquer $x_{1}, \ldots, x_{n} \in R$, podemos determinar um único $R$-homomorfismo tal que $x_{i}=f\left(e_{i}\right)$ para todo $i \in\{1, \ldots, n\}$. Dessa forma, temos a seguinte definição:

Definição 2.5.5. Dada uma sequência $x=\left(x_{1}, \ldots, x_{n}\right)$ de $R$, encontramos um $R$-homomorfismo $f$, como feito acima. Chamamos de complexo de Koszul de $x$ o complexo $K_{\bullet}(x):=K_{\bullet}(f)$ e chamamos de homologia de Koszul de $x$ a homologia $H_{\bullet}(x)=H_{\bullet}(f)$.

Definição 2.5.6. Dada uma sequência $x=\left(x_{1}, \ldots, x_{n}\right)$ de $R$, encontramos um R-homomorfismo $f$, como feito acima. Chamamos o complexo $K_{\bullet}(x, N):=K_{\bullet}(f, N)$ de complexo de Koszul de $x$ com coeficientes em $N$ e a homologia $H_{\bullet}(x, N)=H_{\bullet}(f, N)$ desse complexo é chamada de homologia de Koszul de $x$ com coeficientes em $N$.

Definição 2.5.7. Sejam $x=\left(x_{1}, \ldots, x_{n}\right)$ uma sequência de elementos num anel $R$ e $N$ um $R$ módulo. Dizemos que tal sequência é intersecção completa para $N$ se

$$
H_{\bullet}(x, N)=0 \quad \forall n>0
$$

Observação 2.5.2. A definição acima foi retirada da página 157 de Bourbaki [2006] (complètement sécante significa intersecção completa).

\subsection{Geometria algébrica}

Seja $k$ um corpo algebricamente fechado de característica zero (por exemplo, $\mathbb{C}$ ).

Definição 2.6.1. Chamamos $k^{n}$ de espaço afim (de dimensão n) e o denotamos por $\mathbb{A}^{n}$.

$k^{n}$ é um $k$-espaço vetorial, mas, nesse momento, não usaremos esse fato.

Definição 2.6.2. Seja $S \subset k\left[x_{1}, \ldots, x_{n}\right]$. Definamos o conjunto de zeros de $S$ como:

$$
\mathcal{V}(S):=\left\{\left(a_{1}, \ldots, a_{n}\right) \in \mathbb{A}^{n} \mid f\left(a_{1}, \ldots, a_{n}\right)=0, \quad \forall f \in S\right\} .
$$

Este conjunto é chamado de variedade algébrica afim associada ao conjunto $S$ (ou simplesmente variedade algébrica). Dizemos que $X \subset \mathbb{A}^{n}$, é um conjunto algébrico (ou variedade algébrica) se $X=\mathcal{V}(S)$ para algum $S \subset k\left[x_{1}, \ldots, x_{n}\right]$. Chamamos os polinômios de $S$ de geradores de $\mathcal{V}(S)$.

Se $X=\mathcal{V}(S)$ para algum $S \subset k\left[x_{1}, \ldots, x_{n}\right]$ e $f \in k\left[x_{1}, \ldots, x_{n}\right]$, denotamos por $X(f)$ ou $V(S)(f)$ a variedade algébrica $V(S \cup\{f\})$.

Observação 2.6.1. Seja $S \subset k\left[x_{1}, \ldots, x_{n}\right]$ e $I$ o ideal gerado por $S$. É fácil ver que $\mathcal{V}(S)=\mathcal{V}(I)$. Como $k\left[x_{1}, \ldots, x_{n}\right]$ é um anel Noetheriano, I é finitamente gerado, ou seja, existem $f_{1}, \ldots, f_{m} \in k\left[x_{1}, \ldots, x_{n}\right]$ tais que $I=\left\langle f_{1}, \ldots, f_{m}\right\rangle$. Assim, temos $\mathcal{V}(S)=\mathcal{V}\left(\left\{f_{1}, \ldots, f_{m}\right\}\right)$.

Definição 2.6.3. Seja $R$ um anel (comutativo) e I um ideal de $R$. Chamamos de radical de I o conjunto

$$
\sqrt{I}=\left\{x \in R \mid x^{n} \in I, \text { para algum } n \neq 0\right\} .
$$

Dizemos que I é radical se $\sqrt{I}=I$. 
Definição 2.6.4. Seja $X \subset \mathbb{A}_{n}$. Podemos definir o ideal

$$
\mathcal{I}(X):=\left\{f \in k\left[x_{1}, \ldots, x_{n}\right] \mid f(x)=0, \quad \forall x \in X\right\}
$$

chamado de ideal associado a X.

Observação 2.6.2. Claramente $X \subset \mathcal{V}(\mathcal{I}(X))$, para todo $X \subset \mathbb{A}_{n}$, e $I \subset \mathcal{I}(\mathcal{V}(I))$, para todo ideal $I$ de $k\left[x_{1}, \ldots, x_{n}\right]$. Também podemos concluir que $\mathcal{I}(X)$ é um ideal radical.

Teorema 2.6.1. (Teorema dos zeros de Hilbert)

Seja I um ideal de $k\left[x_{1}, \ldots, x_{n}\right]$, então $\sqrt{I}=\mathcal{I}(\mathcal{V}(I))$.

Demonstração. A demonstração pode ser encontrada em Humphreys [1998] (primeiro teorema do capítulo 1) e em Tauvel e Yu [2005] (página 132).

Do teorema acima concluimos que, se $I$ é um ideal radical, então $I=\sqrt{I}=\mathcal{I}(\mathcal{V}(I))$.

Proposição 2.6.1. Seja $X \subset \mathbb{A}^{n}$ uma variedade algébrica. Então $\mathcal{V}(\mathcal{I}(X))=X$.

Demonstração. Claramente, pelas definições de $\mathcal{I}$ e $\mathcal{V}, X \subset \mathcal{V}(\mathcal{I}(X))$.

Por outro lado, seja $I$ ideal de $k\left[x_{1}, \ldots, x_{n}\right]$ tal que $X=\mathcal{V}(I)$. Então, $I \subset \mathcal{I}(X)$. É fácil ver que isso implica $\mathcal{V}(\mathcal{I}(X)) \subset \mathcal{V}(I)=X$.

Portanto, $\mathcal{V}(\mathcal{I}(X))=X$.

Dessa forma, temos as seguintes correspondências biunívocas:

$$
\begin{aligned}
& \mathcal{V}:\left\{\text { ideais radicais de } k\left[x_{1}, \ldots, x_{n}\right]\right\} \longrightarrow\left\{\text { variedades algébricas de } \mathbb{A}^{n}\right\} \\
& \mathcal{I}:\left\{\text { variedades algébricas de } \mathbb{A}^{n}\right\} \longrightarrow\left\{\text { ideais radicais de } k\left[x_{1}, \ldots, x_{n}\right]\right\}
\end{aligned}
$$

e uma é a inversa da outra.

Ideais primos são exemplos de ideais radicais. Então podemos pensar na correspondência acima restrita aos ideias primos.

Definição 2.6.5. Uma variedade algébrica $X$ de $\mathbb{A}^{n}$ é dita irredutivel se não existem duas variedades algébricas diferentes de $X$ e não vazias, $X_{1}$ e $X_{2}$, tais que $X=X_{1} \cup X_{2}$.

Proposição 2.6.2. Uma variedade algébrica $X$ é irredutível se e somente se $\mathcal{I}(X)$ é um ideal primo.

Demonstração. A demonstração pode ser encontrada no parágrafo 3 (Algebraic varieties in the affine space) do capítulo 7 (Polynomial and power series rings) de Zariski e Samuel [1960].

Ideais maximais são ideais primos. Assim também podemos pensar na correspondência restrita aos ideais maximais.

Proposição 2.6.3. Seja $a \in \mathbb{A}^{n}$. Então $\mathcal{I}(\{a\})$ é ideal maximal. Por outro lado, se I é um ideal maximal, existe $a \in \mathbb{A}^{n}$ tal que $\mathcal{V}(I)=\{a\}$.

Demonstração. A demonstração pode ser encontrada na página 6 de Humphreys [1972].

Assim, temos as seguinte correspondências biunívoas:

\{ideais radicais de $\left.k\left[x_{1}, \ldots, x_{n}\right]\right\} \underset{\mathcal{I}}{\stackrel{\mathcal{V}}{\rightleftarrows}}\left\{\right.$ variedades algébricas de $\left.\mathbb{A}^{n}\right\}$

\{ideais primos de $\left.k\left[x_{1}, \ldots, x_{n}\right]\right\} \underset{\mathcal{I}}{\stackrel{\mathcal{V}}{\rightleftarrows}}\left\{\right.$ variedades algébricas irredutíveis de $\left.\mathbb{A}^{n}\right\}$

\{ideais maximais de $\left.k\left[x_{1}, \ldots, x_{n}\right]\right\} \underset{\mathcal{I}}{\stackrel{\mathcal{V}}{\rightleftarrows}}\left\{\right.$ pontos de $\left.\mathbb{A}^{n}\right\}$ 
Proposição 2.6.4. (i) $\mathcal{V}\left(k\left[x_{1}, \ldots, x_{n}\right]\right)=\emptyset$ e $\mathcal{V}(\emptyset)=k\left[x_{1}, \ldots, x_{n}\right]$

(ii) Sejam $I$ e $J$ ideais de $k\left[x_{1}, \ldots, x_{n}\right]$. Temos: $\mathcal{V}(I) \cup \mathcal{V}(J)=\mathcal{V}(I \cap J)$.

(iii) Sejam $\left\{I_{s}\right\}_{s \in S}$ ideais de $k\left[x_{1}, \ldots, x_{n}\right]$. Temos: $\bigcap_{s \in S} \mathcal{V}\left(I_{s}\right)=\mathcal{V}\left(\sum_{s \in S} I_{s}\right)$.

Demonstração. A demonstração pode ser encontrada na proposição 11.1.4 de Tauvel e Yu [2005].

Pela proposição anterior, concluimos que podemos definir em $\mathbb{A}^{n}$ uma topologia onde os conjuntos fechados são as variedades algébricas afim. Essa topologia é chamada de topologia de Zariski.

Na proposição a seguir, podemos ver algumas características da topologia de Zariski:

Proposição 2.6.5. (i) Seja $X \subset \mathbb{A}^{n}$. Então o fecho de $X$ é $\mathcal{V}(\mathcal{I}(X))$.

(ii) $\mathbb{A}^{n}$ é um espaço topológico Noetheriano (ou seja, satisfaz a condição de cadeias descendente) cujos pontos são fechados.

(iii) Para todo ideal $I$ de $k\left[x_{1}, \ldots, x_{n}\right]$, o conjunto de componentes irredutiveis de $\mathcal{V}(I)$ é finito e é o conjunto das variedades do tipo $\mathcal{V}(p)$, onde $p$ é um ideal primo minimal de $k\left[x_{1}, \ldots, x_{n}\right]$ que contém $I$.

(iv) Todo conjunto algébrico $X$ pode ser expresso de maneira única como $X=X_{1} \cup \ldots \cup X_{m}$, onde cada $X_{i}$ é uma variedade algébrica irredutivel e $X_{i} \not \subset X_{j}, \forall i \neq j$.

Demonstração. Vide em Tauvel e Yu [2005]:

(i) Proposição 11.2.2,

(ii) Proposição 11.2.3,

(iii) Corolário 11.2.4.

Para a demonstração de (iv) vide proposição 1.5 de Hartshorne [1977].

Definição 2.6.6. Seja $X \in \mathbb{A}^{n}$ um conjunto algébrico afim. Definimos o anel de coordenadas de $X$ como

$$
\mathcal{A}(X):=k\left[x_{1}, \ldots, x_{n}\right] / \mathcal{I}(X) .
$$

Observação 2.6.3. Se $X \in \mathbb{A}^{n}$ um conjunto algébrico afim, então $\mathcal{A}(X)$ é um dominio de integridade. Além disso, $\mathcal{A}(X)$ é uma k-álgebra finitamente gerada. Por outro lado, qualquer $k$-álgebra finitamente gerada que é um dominio é o anel de coordenadas de alguma variedade algébrica afim. (Vide observação 1.4.6 de Hartshorne [1977]. No exercício 1.5 desse mesmo livro, Hartshorne pede para provar essa observação trocando domínio de integridade por não ter elementos nilpotentes.)

Definição 2.6.7. Uma k-álgebra associativa, comutativa e finitamente gerada é chamada de $k$ álgebra afim. Uma k-álgebra afim é chamada de reduzida se não tem elementos nilpotentes.

Definição 2.6.8. Seja $X$ uma variedade algébrica afim. Definimos a dimensão de $X$ sendo o supremo dos inteiros $m$ tais que existe uma cadeia de subconjuntos irredutiveis fechados de $X$ distintos $X_{0} \subset X_{1} \subset \ldots \subset X_{m}$. Denotamos a dimensão de $X$ por dimX.

Definição 2.6.9. Em um anel $R$, a altura de um ideal primo $p$ é o supremo dos inteiros $m$ tais que existe uma cadeia de ideais primos distintos $p_{0} \subset p_{1} \subset \ldots \subset p_{m}=p . A$ dimensão (dimensão de Krull) de $R$ é o supremo do conjunto das alturas dos ideais primos de $R$. Denotamos a dimensão de $R$ por $\operatorname{dim} R$.

Proposição 2.6.6. $\operatorname{dimk}\left[x_{1}, \ldots, x_{n}\right]=n$. 
Proposição 2.6.7. Se $X$ é um conjunto algébrico afim, então a dimensão de $X$ é igual a dimensão do anel de coordenadas de $X$.

Demonstração. Vide proposição 1.7 de Hartshorne [1977].

Definição 2.6.10. Uma variedade é dita equidimensional se todas suas componentes irredutíveis têm a mesma dimensão.

Exemplo 2.6.1. Considere a variedade algébrica $\mathcal{V}(x y, x z) \subset \mathbb{A}^{3}$. Claramente temos $\mathcal{V}(x y, x z)=$ $=\mathcal{V}(x) \cup \mathcal{V}(y, z)$. Note que os ideais $\langle x\rangle$ e $\langle y, z\rangle$ são primos, então $\mathcal{V}(x)$ e $\mathcal{V}(y, z)$ são irredutiveis. Além $\operatorname{disso,} \operatorname{dim}(\mathcal{A}(\mathcal{V}(X)))=\operatorname{dim}(k[x, y, z] /\langle x\rangle)=\operatorname{dim}(k[y, z])=2$ e $\operatorname{dim}(\mathcal{A}(\mathcal{V}(y, z)))=$ $=\operatorname{dim}(k[x, y, z] /\langle y, z\rangle)=\operatorname{dim}(k[x])=1$. Portanto, a variedade algébrica $\mathcal{V}(x y, x z)$ não é equidimensional.

\subsection{Sequências regulares}

Definição 2.7.1. Seja $R$ um anel e $M$ um $R$-módulo. Dizemos que $x \in R$ é M-regular (ou regular em $M)$ se $x m \neq 0$ para todo $0 \neq m \in M$.

Definição 2.7.2. Seja $R$ um anel e $M$ um $R$-módulo. Dizemos que uma sequência $x_{1}, \ldots, x_{m}$ de elementos de $R$ é uma sequência M-regular se as seguintes duas condiçôes são satisfeitas:

(i) $x_{1}$ é $M$-regular e $x_{i}$ é $\left(M / \sum_{l=1}^{i-1} x_{l} M\right)$-regular para todo $i \in\{2, \ldots, m\}$,

(ii) $M / \sum_{l=1}^{m} x_{l} M \neq 0$.

Observação 2.7.1. Consultando livros, achei definições diferentes. Alguns autores definem sequência $M$-regular considerando R-módulos (por exemplo Matsumura [1989], de onde foi tirada a definição acima, ou Eisenbud [1995], notando que a condição ii) acima é equivalente a dizer que $\left.\sum_{l=1}^{m} x_{l} M \neq M\right)$. Outros definem sequência regular considerando só anéis $R$, onde sequência regular é uma sequência $M$-regular com $M=R$ (por exemplo, Lam [1999]). Em Futorny e Ovsienko [2003], a definião é dada considerando k-álgebras afins $\Lambda$ e, em vez de usar ii), é usada uma afirmação equivalente: $x_{1}$ não é invertivel em $\Lambda$ e $x_{i}$ não é invertivel em $\Lambda /<x_{1}, \ldots, x_{i-1}>$ para todo $i \in\{2, \ldots, m\}$. Como toda $k$-álgebra afim é um $k$-módulo e um anel, qualquer um dos livros citados acima podem ser tranquilamente consultados.

Observação 2.7.2. A ordem dos elementos em uma sequência regular é importante. Permutando os elementos de uma sequência regular, pode-se obter uma sequência que não é regular. Exemplo: Seja $k$ um corpo e considere $R=M=k[x, y, z]$. A sequência $x(y-1), y, z(y-1)$ é regular pois:

- $x(y-1)$ não é divisor de zero pois $k[x, y, z]$ é domínio de integridade,

- y não é divisor de zero em $k[x, y, z] /\langle x(y-1)\rangle$ pois se temos $y f=g x(y-1)$, para $f, g \in$ $k[x, y, z]$, então $x(y-1)$ divide $f$ e, consequentemente $f \in\langle x(y-1)\rangle$,

- $z(y-1)$ não é divisor de zero em $k[x, y, z] /\langle x(y-1), y\rangle=k[x, y, z] /\langle x, y\rangle \cong k[z]$, que é um dominio de integridade.

- $1 \notin\langle x(y-1), y, z(y-1)\rangle$, então $1 \in k[x, y, z] /\langle x(y-1), y, z(y-1)\rangle e$ $k[x, y, z] /\langle x(y-1), y, z(y-1)\rangle \neq 0$.

Mas $x(y-1), z(y-1)$, y não é uma sequência regular pois $x z(y-1)=z(x(y-1))$, ou seja, $z(y-1)$ é divisor de zero em $k[x, y, z] /\langle x(y-1)\rangle$. 
Proposição 2.7.1. Sejam $R$ um anel Noetheriano e $x_{1}, \ldots, x_{m}$ uma sequência regular em $R$. Se $R$ é um anel graduado e cada $x_{i}$ é homogêneo de grau positivo, então qualquer permutação de $x_{1}, \ldots, x_{m}$ é também regular.

Demonstração. Ver teorema 28 em Matsumura [1970] página 102 ou Matsumura [1989] página 127.

Proposição 2.7.2. Sejam $\Lambda$ uma álgebra afim de dimensão $n$ e $x_{1}, \ldots, x_{t}$ uma sequência de elementos de $\Lambda$, onde $1 \leq t \leq n$.

(i) Se $\Lambda$ é graduada e $x_{1}, \ldots, x_{t}$ são homogêneos, então $x_{1}, \ldots, x_{t}$ é uma sequência regular em $\Lambda$ se,e somente se, a sequência $x_{1}, \ldots, x_{t}$ é uma interseção completa em $\Lambda$.

(ii) Se $\Lambda$ é Cohen-Macaulay, então a sequência $x_{1}, \ldots, x_{t}$ é interseção completa para $\Lambda$ se, e somente se, a variedade $\mathcal{V}\left(x_{1}, \ldots, x_{t}\right)$ é equidimensional de dimensão $n-t$.

Demonstração. Proposição 2.1 de Futorny e Ovsienko [2003].

Observação 2.7.3. Para detalhes sobre anéis Cohen-Macaulay ver página 134 de Matsumura [1989]. Neste trabalho, só vamos usar o fato que o anel de polinômios é Cohen-Macaulay (teorema 17.7 de Matsumura [1989]).

Proposição 2.7.3. Seja $A=k\left[x_{1}, \ldots, x_{n}\right]$ a álgebra de polinômios e $g_{1}, \ldots, g_{t} \in A$. A sequência $x_{1}, \ldots, x_{n}, g_{1}, \ldots, g_{t}$ é uma intersecçáo completa para $A$ se, e somente se, a sequência $g_{1}, \ldots, g_{t}$ é uma interseç̧ão completa para $k\left[x_{r+1}, \ldots, x_{n}\right]$, onde $g_{i}\left(x_{r+1}, \ldots, x_{n}\right)=g_{i}\left(0, \ldots, 0, x_{r+1}, \ldots, x_{n}\right)$ para $i \in\{1, \ldots, t\}$.

Demonstração. Lema 2.1 de Futorny e Ovsienko [2003].

\subsection{Provando equidimensionalidade}

Considere $k\left[x_{1}, \ldots, x_{n}\right]$. Sejam $y_{1}, \ldots, y_{k}$ distintos com $\left\{y_{1}, \ldots, y_{k}\right\} \subset\left\{x_{1}, \ldots, x_{n}\right\}$. A variedade $\mathcal{V}\left(y_{1}, \ldots, y_{k}\right)$ é irredutível e tem dimensão $n-k$, pois:

- o ideal $<y_{1}, \ldots, y_{k}>$ é primo

- $\mathcal{A}\left(\mathcal{V}\left(y_{1}, \ldots, y_{k}\right)\right)=k\left[x_{1}, \ldots, x_{n}\right] /<y_{1}, \ldots, y_{k}>\cong k[S]$ onde $S=\left\{x_{1}, \ldots, x_{n}\right\} \backslash\left\{y_{1}, \ldots, y_{k}\right\}$ e $\operatorname{dim}(k[S])=n-k$.

Para provarmos que uma variedade é equidimensional, usaremos 3 processos:

1. Isomorfismos: Sejam $V=\mathcal{V}(S), U=\mathcal{V}(R) \subset \mathbb{A}^{n}$ variedades algébricas. Se $V$ é uma variedade isomórfica a variedade $U$ e $U$ é equidimensional de dimensão $m$ então $V$ é equidimensional de dimensão $m$. Para provar que $V$ e $U$ são isomórficos basta achar um automorfismo $f$ de $k\left[x_{1}, \ldots, x_{n}\right]$ tal que $f(S)=R$.

2. União de subvariedades: Sejam $V, V_{1}, \ldots, V_{k} \subset \mathbb{A}^{n}$ variedades tais que $V=V_{1} \cup \ldots \cup V_{k}$. $V$ é equidimensional de dimensão $m$ se e somente se $V_{1}, \ldots, V_{k}$ são equidimensionais de dimensão $m$. Isso ocorre porque toda variedade irredutível contida em algum $V_{i}, i \in\{1, \ldots, k\}$, é uma variedade irredutível de $V$ e toda variedade irredutível de $V$ tem que estar contida em algum $V_{i}$ (já que é irredutível).

3. Acrescentar variável: $\operatorname{Seja} \mathcal{V}\left(f_{1}, \ldots, f_{k}\right) \subset \mathbb{A}^{n}$ e $y \in\left\{x_{1}, \ldots, x_{n}\right\}$. Se $\mathcal{V}\left(f_{1}, \ldots, f_{k}, y\right)$ é equidimensional de dimensão $n-k-1$, então $\mathcal{V}\left(f_{1}, \ldots, f_{k}\right)$ é equidimensional de dimensão $n-k$. Esse fato é consequência das proposições 2.7.1 e 2.7.2.

Exemplo 2.8.1. Sejam $y_{1}, \ldots, y_{k}, y_{k+1}$ distintos com $\left\{y_{1}, \ldots, y_{k}, y_{k+1}\right\} \subset\left\{x_{1}, \ldots, x_{n}\right\}$. A variedade $\mathcal{V}\left(y_{1}, \ldots, y_{k} y_{k+1}\right)$ é equidimensional de dimensão $n-k$ pois:

$$
\mathcal{V}\left(y_{1}, \ldots, y_{k} y_{k+1}\right)=\mathcal{V}\left(y_{1}, \ldots, y_{k-1}, y_{k}\right) \cup \mathcal{V}\left(y_{1}, \ldots, y_{k-1}, y_{k+1}\right)
$$




\section{Capítulo 3}

\section{Subálgebra de Mishchenko-Fomenko}

Esse capítulo é baseado em Futorny e Molev [2015] e Molev [2018].

\subsection{Definição}

Seja $\mathfrak{g}$ uma álgebra de Lie simples sobre $\mathbb{C}$. Seja $\left\{Y_{1}, \ldots, Y_{l}\right\}$ uma base de $\mathfrak{g}$. Podemos definir na álgebra simétrica $\mathrm{S}(\mathfrak{g})$ um colchete de Lie-Poisson $\{$,$\} . Sabemos que \mathrm{S}(\mathfrak{g}) \cong \mathbb{C}\left[Y_{1}, \ldots, Y_{l}\right]$ (álgebra de polinômios nas variáveis $Y_{1}, \ldots, Y_{l}$, proposição 2.3.4). Então, para $f, g \in \mathbb{C}\left[Y_{1}, \ldots, Y_{l}\right]$, defina:

$$
\{f, g\}=\sum_{i, j=1}^{l}\left[Y_{i}, Y_{j}\right] \frac{\partial f}{\partial Y_{i}} \frac{\partial g}{\partial Y_{j}}
$$

Note que $\left\{Y_{i}, Y_{j}\right\}=\left[Y_{i}, Y_{j}\right]$ e essa é a única maneira de definir $\{$,$\} de forma que isso ocorra.$

A subálgebra $\{P \in \mathrm{S}(\mathfrak{g}) \mid\{x, P\}=0 \quad \forall x \in \mathfrak{g}\}$ de $\mathrm{S}(\mathfrak{g})$ é a álgebra de polinômios $\mathfrak{g}$-invariantes de $\mathrm{S}(\mathfrak{g})$ e é denotada por $\mathrm{S}(\mathfrak{g})^{\mathfrak{g}}$.

Seja $P\left(Y_{1}, \ldots, Y_{l}\right) \in \mathrm{S}(\mathfrak{g})$ um elemento de grau $d$. Fixe $\mu \in \mathfrak{g}^{*}$. Seja $z$ uma variável. Substitua $Y_{i}$ por $Y_{i}+z \mu\left(Y_{i}\right)$ em $P$ e expanda o resultado para um polinômio em $z$,

$$
P\left(Y_{1}+z \mu\left(Y_{1}\right), \ldots, Y_{l}+z \mu\left(Y_{l}\right)\right)=P_{\mu}^{(0)}+P_{\mu}^{(1)} z+\cdots P_{\mu}^{(d)} z^{d}
$$

definindo assim elementos $P_{\mu}^{(i)} \in \mathrm{S}(\mathfrak{g})$, chamados $\mu$-deslocamentos de $P$. Denotamos por $\overline{\mathcal{A}}_{\mu}$ a subálgebra de $\mathrm{S}(\mathfrak{g})$ gerada pelos $\mu$-deslocamentos de todos polinômios $\mathfrak{g}$-invariantes $P$. A subálgebra $\overline{\mathcal{A}}_{\mu}$ é chamada de subálgebra de deslocamento de argumento ou subálgebra de Mishchenko-Fomenko. Para todo $\mu \in \mathfrak{g}^{*}, \overline{\mathcal{A}}_{\mu}$ é comutativa em relação ao colchete de Poisson-Lie (ou seja, para todo $R, S \in \mathrm{S}(\mathfrak{g}),\{R, S\}=0)$. Isso é consequência do seguinte lema:

Lema 3.1.1. Sejam $P_{1}, \ldots, P_{m} \in S(\mathfrak{g})^{\mathfrak{g}}$. Então, para todo $\mu \in \mathfrak{g}^{*}$, os polinômios

$$
\left\{P_{i, \mu}^{(j)} \mid i=1, \ldots, m \quad e \quad j=1, \ldots, \operatorname{deg}\left(P_{i, \mu}^{(j)}\right)-1\right\}
$$

são mutuamente comutativos com respeito ao colchete de Poisson-Lie.

Demonstração. Ver Mishchenko e Fomenko [1978].

Definição 3.1.1. Seja $k$ um corpo algebricamente fechado de característica zero e seja $\mathfrak{g}$ uma $k$-álgebra de Lie de dimensão finita. Para cada $\mu \in \mathfrak{g}^{*}$ o subespaço

$$
\mathfrak{g}_{\mu}=\{x \in \mathfrak{g} \mid \mu([x, y])=0 \text { para todo } y \in \mathfrak{g}\}
$$

é chamado de estabilizador de $\mu$. O valor mínimo do conjunto $\left\{\right.$ dimg $\left.\mathfrak{g}_{\mu}: \mu \in \mathfrak{g}^{*}\right\}$ é chamado de índice

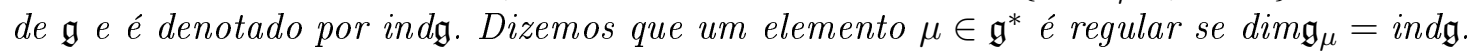


Se $\mathfrak{g}$ for redutível (por exemplo, semissimples), ind $\mathfrak{g}$ é igual ao rank de $\mathfrak{g}$.

Vamos denotar por $\mathfrak{g}_{\text {reg }}^{*}$ O conjunto de todos os elementos regulares de $\mathfrak{g}^{*}$.

Teorema 3.1.1. Seja $k$ um corpo algebricamente fechado de característica zero e seja $\mathfrak{g}$ uma $k$ álgebra de Lie de dimensão finita. Suponha que

(i) a subálgebra de polinômios $\mathfrak{g}$-invariantes, $S(\mathfrak{g})^{\mathfrak{g}}$, contém polinômios algebricamente independentes $f_{1}, \ldots, f_{l}$, com $l=$ ind $\mathfrak{g}$, tais que $\sum_{i=1}^{l} \operatorname{deg} f_{i}=\frac{1}{2}(\operatorname{dim} \mathfrak{g}+\operatorname{ind} \mathfrak{g}):=b(\mathfrak{g}) e$

(ii) $\operatorname{codim}\left(\mathfrak{g}^{*} \backslash \mathfrak{g}_{\text {reg }}^{*}\right) \geq 3$

então, para todo $\mu \in \mathfrak{g}_{\text {reg }}^{*}$, a subalgebra $\bar{A}_{\mu}$ é uma álgebra de polinômios de dimensão de Krull b(g) e é uma subálgebra maximal Poisson comutativa de $S(\mathfrak{g})$.

Demonstração. Ver Panyushev e Yakimova [2008].

Para álgebras de Lie semissimples, (ii) ocorre e (i) segue do Teorema 7.3.8 de Dixmier [1977]. Então, nesse caso, $\bar{A}_{\mu}$ é uma subálgebra maximal Poisson comutativa de $\mathrm{S}(\mathfrak{g})$. Isso já havia sido provado em Tarasov [2002].

Teorema 3.1.2. Seja $\mathfrak{g}$ uma álgebra de Lie simples com dimensão finita. Seja $\mu \in \mathfrak{g}^{*}$ regular. Se $P_{1}, \ldots, P_{n}(n=i n d \mathfrak{g})$ são geradores algebricamente independentes de $S(\mathfrak{g})^{\mathfrak{g}}$ com graus $d_{1}, \ldots, d_{n}$, respectivamente, então

$$
\left\{P_{i, \mu}^{(j)} \mid i=1, \ldots, n \quad e \quad j=0,1, \ldots, d_{j}-1\right\}
$$

é um conjunto de geradores algebricamente independentes de $\overline{\mathcal{A}}_{\mu}$.

Demonstração. Ver [Bolsinov, 1991]. O caso $\mu$ regular e $\mathfrak{g}$ semissimples está provado em Mishchenko e Fomenko [1978]. Outra demonstração pode ser vista em Feigin et al. [2010].

\subsection{Problema de Vinberg}

A álgebra envolvente universal $\mathrm{U}(\mathfrak{g})$ de uma álgebra de Lie $\mathfrak{g}$ tem uma filtração natural e a álgebra graduada $\operatorname{gr} U(\mathfrak{g})$ associada a essa filtração é isomórfica a $\mathrm{S}(\mathfrak{g})$. Se $\mathcal{A}$ é uma subálgebra comutativa de $\mathrm{U}(\mathfrak{g})$ então gr $\mathcal{A}$ é uma subálgebra Poisson comutativa de $\mathrm{S}(\mathfrak{g})$. $\overline{\mathcal{A}}_{\mu}$ é uma subálgebra Poisson comutativa de $\mathrm{S}(\mathfrak{g})$, então podemos nos perguntar se existe uma subálgebra comutativa $\mathcal{A}_{\mu}$ de $\mathrm{U}(\mathfrak{g})$ tal que gr $\mathcal{A}_{\mu}=\overline{\mathcal{A}}_{\mu}$. Esse problema foi proposto por Vinberg em Vinberg [1991], onde ele produz famílias comutativas de elementos de $\mathrm{U}(\mathfrak{g})$. Soluções positivas para o problema de Vinberg foram dadas em Rybnikov [2006] (para $\mu$ regular e semissimples) e em Feigin et al. [2010] (para qualquer $\mu$ regular).

\subsubsection{A solução de Feigin, Frenkel e Toledano-Laredo}

A solução do problema de Vinberg apresentada por Feigin, Frenkel e Toledano-Laredo em Feigin et al. [2010] usa o Centro de Feigin-Frenkel.

Seja $\mathfrak{g}$ uma álgebra de Lie simples sobre $\mathbb{C}$. Seja $\langle$,$\rangle a forma bilinear simétrica invariante$ chamada forma de Killing normalizada que é definida por:

$$
\langle X, Y\rangle=\frac{1}{2 h^{\vee}} \operatorname{tr}(\operatorname{ad} X \operatorname{ad} Y)
$$

onde $h^{\vee}$ é o número de Coxeter dual de $\mathfrak{g}$.

A álgebra de Kac-Moody afim $\widehat{\mathfrak{g}}$ correspondente a $\mathfrak{g} \mathrm{e}\langle$,$\rangle é a extensão central$

$$
\widehat{\mathfrak{g}}=\mathfrak{g}\left[t, t^{-1}\right] \oplus \mathbb{C} K
$$


onde $\mathfrak{g}\left[t, t^{-1}\right]$ é a álgebra dos polinômios de Laurent em $t$ com coeficientes em $\mathfrak{g}$. Para $X \in \mathfrak{g}$ e $r \in \mathbb{Z}$, escreva $X[r]$ para $X t^{r}$. O colchete de Lie de $\widehat{\mathfrak{g}}$ é definido por

$$
[X[r]+Y[s]]=[X, Y][r+s]+r \delta_{r,-s}\langle X, Y\rangle K \quad X, Y \in \mathfrak{g}, \quad r, s \in \mathbb{Z}
$$

e o elemento $K$ é central em $\widehat{\mathfrak{g}}$.

Para todo $\kappa \in \mathbb{C}$, denote por $\mathrm{U}_{\kappa}(\widehat{\mathfrak{g}})$ o quociente da álgebra $\mathrm{U}(\widehat{\mathfrak{g}})$ pelo ideal gerado por $K-\kappa$, chamado de álgebra envolvente universal no nivel $\kappa$. Seja $I$ o ideal à esquerda de $\mathrm{U}_{\kappa}(\widehat{\mathfrak{g}})$ gerado por $\mathfrak{g}[t]$. O módulo vácuo de nível $\kappa$ sobre $\widehat{g}$ é o quociente

$$
V_{\kappa}(\mathfrak{g})=\mathrm{U}_{\kappa}(\widehat{\mathfrak{g}}) / I .
$$

Quando $\kappa=-h^{\vee}$, falamos que $V_{\kappa}(\mathfrak{g})$ é módulo vácuo de nível crítico.

Definição 3.2.1. O centro de Feigin-Frenkel é a álgebra definida por:

$$
\mathfrak{z}(\widehat{\mathfrak{g}})=\left\{v \in V_{-h^{\vee}}(\mathfrak{g}) \mid g[t] v=0\right\} .
$$

Proposição 3.2.1. O centro de Feigin-Frenkel coincide com a álgebra associativa NormI/I, onde $I$ é o ideal à esquerda de $U_{h^{\vee}}(\mathfrak{g})$ gerado por $\mathfrak{g}[t]$ e $N o r m I=\left\{v \in U_{-h^{\vee}}(\mathfrak{g}) \mid I v \subset I\right\}$ (normalizador de I em $\left.U_{-h^{\vee}}(\mathfrak{g})\right)$.

Demonstração. $\quad \mathfrak{z}(\widehat{\mathfrak{g}}) \subset \operatorname{Norm} I / I$ :

Seja $v \in \mathfrak{z}(\widehat{\mathfrak{g}})$. Então $v=x+I$ onde $x \in \mathrm{U}_{\kappa}(\widehat{\mathfrak{g}})$. Seja $i \in I$. Temos: $i v=i(x+I)=i x+I=I$. Assim $I v \subset I$ e $v \in \operatorname{Norm} I / I$.

- $\operatorname{Norm} I / I \subset \mathfrak{z}(\widehat{\mathfrak{g}})$ :

Seja $v \in \operatorname{Norm} I / I$. Então existe $x \in \operatorname{Norm} I$ tal que $v=x+I$. Temos: $\mathfrak{g}[t] v=\mathfrak{g}[t](x+I)=$ $\mathfrak{g}[t] x+I=I=0$, pois $I$ é gerado por $\mathfrak{g}[t]$. Portanto, $v \in \mathfrak{z}(\widehat{\mathfrak{g}})$.

Pelo teorema de Poincaré-Birkhoff-Witt, $V_{-h^{\vee}}(\mathfrak{g})$ é isomórfico, como um espaço vetorial, à álgebra envolvente universal $\mathrm{U}\left(t^{-1} \mathfrak{g}\left[t^{-1}\right]\right)$. A aplicação $\mathfrak{z}(\widehat{\mathfrak{g}}) \hookrightarrow \mathrm{U}\left(t^{-1} \mathfrak{g}\left[t^{-1}\right]\right)$ é um mergulho de álgebras, logo $\mathfrak{z}(\widehat{\mathfrak{g}})$ pode ser visto como uma subálgebra de $\mathrm{U}\left(t^{-1} \mathfrak{g}\left[t^{-1}\right]\right) . V_{-h^{\vee}}$ pode ser munido de uma estrutura de álgebra vertex afim e, dessa forma, $\mathfrak{z}(\widehat{\mathfrak{g}})$ é o centro dessa álgebra vertex afim. Isso nos permite concluir que $\mathfrak{z}(\widehat{\mathfrak{g}})$ é comutativa. Qualquer elemento de $\mathfrak{z}(\widehat{\mathfrak{g}})$ é chamado de vetor de Segal-Sugawara.

Seja $T$ a derivação de $\mathrm{U}\left(t^{-1} \mathfrak{g}\left[t^{-1}\right]\right)$ tal que $T(X[r])=r X[r-1]$, para todo $X \in \mathfrak{g}$ e $r<0$.

Teorema 3.2.1. (Feigin e Frenkel [1992]) Existem vetores de Segal-Sugawara $S_{1}, \ldots, S_{n}(n=i n d \mathfrak{g})$ tais que

$$
\mathfrak{z}(\widehat{\mathfrak{g}})=\mathbb{C}\left[T^{r} S_{i} \mid i=1, \ldots, n, r \geq 0\right] .
$$

Demonstração. Os detalhes da prova ficam em Frenkel [2007].

A família $S_{1}, \ldots, S_{n}$ do teorema anterior é chamada de conjunto completo de vetores de SegalSugawara.

Considere agora a derivação $D$ de $\mathrm{U}\left(t^{-1} \mathfrak{g}\left[t^{-1}\right]\right)$ tal que $D(X[r])=r X[r]$, para todo $X \in \mathfrak{g}$ e $r<0$. $D$ define uma graduação na álgebra $\mathrm{U}\left(t^{-1} \mathfrak{g}\left[t^{-1}\right]\right)$.

Dado $\mu \in \mathfrak{g}^{*}$ e um elemento $z \in \mathbb{C}$ diferente de 0 , a função:

$$
\begin{aligned}
\varrho_{\mu, z}: \mathrm{U}\left(t^{-1} \mathfrak{g}\left[t^{-1}\right]\right) & \rightarrow \mathrm{U}(\mathfrak{g}) \\
X[r] & \mapsto X z^{r}+\delta_{r,-1} \mu(X)
\end{aligned}
$$

é um homomorfismo de álgebras. A imagem do centro de Feigin-Frenkel $\mathfrak{z}(\widehat{\mathfrak{g}})$ por $\varrho_{\mu, z}$ é uma álgebra comutativa de $\mathrm{U}(\mathfrak{g})$, que não depende de $z$, e é denotada por $A_{\mu}$. Se $S \in \mathrm{U}\left(t^{-1} \mathfrak{g}\left[t^{-1}\right]\right)$ é um elemento 
de grau $d$ em relação a graduação definida por $D$, então, considerando que $\varrho_{\mu, z}(S)$ é um polinômio em $z^{-1}$, podemos definir elementos $S^{(i)} \in \mathrm{U}(\mathfrak{g})$ de forma que:

$$
\varrho_{\mu, z}(S)=S^{(0)} z^{-d}+\cdots+S^{(d-1)} z^{-1}+S^{(d)} .
$$

Proposição 3.2.2. Para todo $\mu \in \mathfrak{g}^{*}$ :

(i) $\overline{A_{\mu}} \subset g r A_{\mu}$.

Para todo $\mu \in \mathfrak{g}^{*}$ regular:

(ii) a subálgebra $A_{\mu}$ de $U(\mathfrak{g})$ é maximal comutativa,

(iii) se $\left\{S_{1}, \ldots, S_{n}\right\} \subset \mathfrak{z}(\widehat{\mathfrak{g}})$ é um conjunto completo de vetores de Segal-Sugawara, onde $S_{i}$ tem grau $d_{i}$, para todo $i \in\{1, \ldots, n\}$, então os elementos $S_{k}^{(i)}$, onde $k \in\{1, \ldots, n\}$ e $i \in\left\{0,1, \ldots, d_{k}-1\right\}$, são geradores algebricamente independentes de $A_{\mu}$,

(iv) $g r A_{\mu}=\overline{A_{\mu}}$.

Demonstração. Ver Feigin et al. [2010].

\subsection{Alguns conceitos}

Nessa seção definiremos alguns conceitos e notações que serão necessários para entender alguns geradores do centro de Feigin-Frenkel e da subálgebra $A_{\mu}$ que serão estudados na próxima seção.

Dada uma álgebra associativa com unidade $\mathcal{A}$ e uma matriz $M=\left(M_{i j}\right) n \times n$ com entradas em $\mathcal{A}$ podemos definir o elemento

$$
M=\sum_{i, j=1}^{n} e_{i j} \otimes M_{i j}
$$

em End $\mathbb{C}^{n} \otimes \mathcal{A}$, onde $e_{i j}$ formam a base canônica de End $\mathbb{C}^{n}$. E, dados $m$ inteiro positivo e $a \in$ $\{1, \ldots, m\}$, defina

$$
M_{a}=\sum_{i, j=1}^{n} 1^{\otimes(a-1)} \otimes e_{i j} \otimes 1^{\otimes(m-a)} \otimes M_{i j}
$$

em $\left(\operatorname{End} \mathbb{C}^{n}\right)^{\otimes m} \otimes \mathcal{A}=\underbrace{\operatorname{End} \mathbb{C}^{n} \otimes \cdots \otimes \operatorname{End} \mathbb{C}^{n}}_{m} \otimes \mathcal{A}$.

Agora considere $\mathbb{C}\left[S_{m}\right]$, a álgebra do grupo simétrico $S_{m}$.

Definição 3.3.1. Chamamos de simetrizador o seguinte elemento de $\mathbb{C}\left[S_{m}\right]$ :

$$
h^{(m)}=\frac{1}{m !} \sum_{s \in S_{m}} s .
$$

Definição 3.3.2. Chamamos de antissimetrizador o seguinte elemento de $\mathbb{C}\left[S_{m}\right]$ :

$$
a^{(m)}=\frac{1}{m !} \sum_{s \in S_{m}} \text { sgns } \cdot s .
$$

Sejam $a, b \in\{1, \ldots, m\}$, com $a<b$. Denote por $s_{a b}$ a transposição $(a b) \in S_{m}$. Defina o seguinte elemento de $\left(\operatorname{End} \mathbb{C}^{n}\right)^{\otimes m}$ :

$$
P_{a b}=\sum_{i, j=1}^{n} 1^{\otimes(a-1)} \otimes e_{i j} \otimes 1^{\otimes(b-a-1)} \otimes e_{j i} \otimes 1^{\otimes(m-b)} .
$$


Note que $P_{a b}\left(v_{1} \otimes \cdots \otimes v_{a} \otimes \cdots \otimes v_{b} \otimes \cdots \otimes v_{m}\right)=v_{1} \otimes \cdots \otimes v_{b} \otimes \cdots \otimes v_{a} \otimes \cdots \otimes v_{m}$, para todo $v_{1} \otimes \cdots \otimes v_{a} \otimes \cdots \otimes v_{b} \otimes \cdots \otimes v_{m} \in \mathbb{C}^{\otimes m}$, pois, se $f_{1}, \ldots, f_{n}$ é a base canônica de $\mathbb{C}^{n}, v_{a}=f_{r}$ e $v_{b}=f_{s}$, temos:

$$
\begin{aligned}
P_{a b}\left(v_{1} \otimes \cdots \otimes f_{r} \otimes \cdots \otimes f_{s} \otimes \cdots \otimes \cdots \otimes v_{m}\right) & =\sum_{i, j=1}^{n} v_{1} \otimes \cdots \otimes e_{i j}\left(f_{r}\right) \otimes \cdots \otimes e_{j i}\left(f_{s}\right) \otimes \cdots \otimes v_{m}= \\
& =\sum_{i, j=1}^{n} v_{1} \otimes \cdots \otimes \delta_{j r} f_{i} \otimes \cdots \otimes \delta_{i s} f_{j} \otimes \cdots \otimes v_{m}= \\
& =v_{1} \otimes \cdots \otimes f_{s} \otimes \cdots \otimes f_{r} \otimes \cdots \otimes v_{m}
\end{aligned}
$$

para todos $r, s \in\{1, \ldots, m\}$.

Podemos definir um homomorfismo de álgebras que vai de $\mathbb{C}\left[S_{m}\right]$ em $\left(\text { End } \mathbb{C}^{n}\right)^{\otimes m}$ e que leva $s_{a b}$ em $P_{a b}$ para toda transposição $s_{a b}$ de $S_{m}$. Denotaremos por $P_{x}$ a imagem de $x \in \mathbb{C}\left[S_{m}\right]$ por esse homomorfismo. Defina $H^{(m)}:=P_{h^{(m)}} \in\left(\operatorname{End}^{n}\right)^{\otimes m}$ e $A^{(m)}:=P_{a^{(m)}} \in\left(\operatorname{End} \mathbb{C}^{n}\right)^{\otimes m}$.

Dado $a \in\{1, \ldots, m\}$, defina a função linear dada por:

$$
\begin{aligned}
\operatorname{tr}_{a}:\left(\operatorname{End} \mathbb{C}^{n}\right)^{\otimes m} & \rightarrow\left(\operatorname{End} \mathbb{C}^{n}\right)^{\otimes m-1} \\
\left(x_{1} \otimes \cdots \otimes x_{m}\right) & \mapsto x_{a}\left(x_{1} \otimes \cdots \otimes x_{a-1} \otimes x_{a+1} \otimes \cdots \otimes x_{m}\right) .
\end{aligned}
$$

Lembre-se que os elementos $x_{a}$ pertencem a $\operatorname{End}^{n}$ então podem ser vistos como matrizes $n \times n$ e ter seu traço calculado.

Denotamos por $\operatorname{tr}_{1, \ldots, m}$ a composta $\operatorname{tr}_{1} \circ \cdots \circ \operatorname{tr}_{m}$.

Dada uma álgebra associativa com unidade U, para $\left(\text { End }^{n}\right)^{\otimes m} \otimes \mathrm{U}$, abusaremos da notação e denotaremos $H^{(m)} \otimes 1 \in\left(\operatorname{End} \mathbb{C}^{n}\right)^{\otimes m} \otimes \mathrm{U}$ por $H^{(m)}, A^{(m)} \otimes 1 \in\left(\operatorname{End} \mathbb{C}^{n}\right)^{\otimes m} \otimes \mathrm{U}$ por $A^{(m)}, \operatorname{tr}_{a} \otimes 1$ : $\left(\operatorname{End}^{n}\right)^{\otimes m} \otimes \mathrm{U} \rightarrow\left(\operatorname{End}^{n}\right)^{\otimes(m-1)} \otimes \mathrm{U}$ por $\operatorname{tr}_{a}$ e $\operatorname{tr}_{1} \otimes 1 \circ \cdots \circ \operatorname{tr}_{m} \otimes 1:\left(\text { End }^{n}\right)^{\otimes m} \otimes \mathrm{U} \rightarrow \mathrm{U}$ por $\operatorname{tr}_{1, \ldots, m}$.

Definição 3.3.3. Uma matriz $M=\left(M_{i j}\right), n \times n$, com entradas em uma $\mathbb{C}$-álgebra associativa é chamada de matriz de Manin se satisfaz as seguintes relaçôes:

$$
M_{i j} M_{k l}-M_{k l} M_{i j}=M_{k j} M_{i l}-M_{i l} M_{k j}
$$

para todos $i, j, k, l \in\{1, \ldots, n\}$.

Proposição 3.3.1. Seja $M$ uma matriz de Manin. Considere a notação $M_{a}$ dada em (3.15). $M_{a} M_{b}$ denota o produto dos elementos $M_{a}$ e $M_{b}$ da álgebra $\left(E n d \mathbb{C}^{n}\right)^{\otimes m} \otimes \mathcal{A}$. Para $m \in\{1, \ldots, n\}$ temos:

$$
\operatorname{tr}_{1, \ldots, m} A^{(m)} M_{1} \cdots M_{m}=\sum_{1 \leq i_{1}<\cdots<i_{m} \leq n} \sum_{\sigma \in S_{m}} \operatorname{sgn} \sigma \cdot M_{i_{\sigma(1)} i_{1}} \cdots M_{i_{\sigma(m)} i_{m}} .
$$

Além disso, para $m \geq 1$ temos:

$$
\operatorname{tr}_{1, \ldots, m} H^{(m)} M_{1} \cdots M_{m}=\sum_{1 \leq i_{1} \leq \cdots \leq i_{m} \leq n} \frac{1}{\alpha_{1} ! \cdots \alpha_{m} !} \sum_{\sigma \in S_{m}} M_{i_{m} i_{\sigma(m)}} \cdots M_{i_{1} i_{\sigma(1)}}
$$

onde $\alpha_{i}$ é a multiplicidade do indice $i \in\{1, \ldots, n\}$ em $\left(i_{1}, \ldots, i_{m}\right)$.

Demonstração. Ver proposição 3.2.2. da página 46 de Molev [2018].

Dada uma matriz $M$, definimos o determinante coluna de $M$ por:

$$
\operatorname{cdet}(M)=\sum_{\sigma \in S_{n}} \operatorname{sgn} \sigma M_{\sigma(1) 1} \cdots M_{\sigma(n) n} .
$$

Assim temos:

$$
\operatorname{cdet}(M)=\operatorname{tr}_{1, \ldots, m} A^{(m)} M_{1} \cdots M_{m}
$$


Corolário 3.3.1. Se $M$ é uma matriz de Manin e u uma variável temos:

$$
\operatorname{cdet}(1+u M)=\sum_{m=0}^{n} u^{m} \operatorname{tr}_{1, \ldots, m} A^{(m)} M_{1} \cdots M_{m} .
$$

Demonstração. Ver Corolário 3.2.5. da página 48 de Molev [2018].

\subsection{Tipo $A$}

Nessa seção vamos focar o estudo para as álgebras de Lie de tipo $A$.

\subsubsection{Geradores do centro de Feigin-Frenkel}

Considere o seguinte homormorfismo sobrejetor:

$$
\begin{aligned}
p: \mathfrak{g l}_{n} & \rightarrow \mathfrak{s l}_{n} \\
X & \mapsto X-\frac{1}{n}(\operatorname{tr} X) I d
\end{aligned}
$$

$p$ induz homomorfismos sobrejetores $\mathrm{U}\left(\mathfrak{g l}_{n}\right) \rightarrow \mathrm{U}\left(\mathfrak{s l}_{n}\right)$ e $S\left(\mathfrak{g l}_{n}\right) \rightarrow S\left(\mathfrak{s l}_{n}\right)$. Esses homormorfismos nos permitem encontrar para qualquer resultado para $\mathfrak{g l}_{n}$ um resultado correspondente para $\mathfrak{s l}_{n}$. Assim, em vez de trabalharmos com as álgebras $\mathfrak{s l}_{n}$ iremos trabalhar com $\mathfrak{g l}_{n}$.

Dada uma forma bilinear simétrica não degenerada $B$ de $\mathfrak{g l}_{n}$, temos que $\mathfrak{g l}_{n} \cong \mathfrak{g l}_{n}^{*}$ através do isomorfismo $f$ que leva $x \in \mathfrak{g l}_{n}$ em $f(x) \in \mathfrak{g l}_{n}^{*}$, onde $f(x)$ é o funcional linear que leva $y \in \mathfrak{g l}_{n} \mathrm{em}$ $B(x, y)$. Dessa forma, veremos todos $\mu \in \mathfrak{g l}_{n}^{*}$ como matrizes $n \times n$.

Denote por $E_{i j}$, para $i, j \in\{1, \ldots, n\}$, os elementos da base canônica de $\mathfrak{g l}_{n}$. Considere a forma bilinear invariante em $\mathfrak{g l}_{n}$ dada por:

$$
\langle X, Y\rangle=\operatorname{tr}(X Y)-\frac{1}{n} \operatorname{tr} X \operatorname{tr} Y
$$

para todos $X, Y \in \mathfrak{g l}_{n}$. Note que o kernel dessa forma é gerado pelo elemento $E_{11}+\cdots+E_{n n}$ e a restrição dela à subálgebra $\mathfrak{s l}_{n}$ é dada por:

$$
\langle X, Y\rangle=\operatorname{tr}(X Y)
$$

para todos $X, Y \in \mathfrak{s l}_{n}$.

O colchete de Lie da álgebra de Kac-Moody afim $\widehat{\mathfrak{g l}}_{n}=\mathfrak{g l}_{n}\left[t, t^{-1}\right] \oplus \mathbb{C} K$ é definido por:

$$
\left[E_{i j}[r], E_{k l}[s]\right]=\delta_{k j} E_{i l}[r+s]-\delta_{i l} E_{k j}[r+s]+r \delta_{r,-s} K\left(\delta_{k j} \delta_{i l}-\frac{\delta_{i j} \delta_{k l}}{n}\right)
$$

onde o elemento $K$ é central em $\widehat{\mathfrak{g l}}_{n}$. O nível crítico $-n$ coincide com o negativo do número de Coxeter dual para $\mathfrak{s l}_{n}$. Com a forma bilinear acima, o centro de Feigin-Frenkel $\mathfrak{z}\left(\widehat{\mathfrak{g l}}_{n}\right)$ coincide com a subálgebra dos elementos $\mathfrak{g l}_{n}[t]$-invariantes e com a subálgebra dos elementos $\mathfrak{s l}_{n}[t]$-invariantes do módulo vácuo:

$$
\mathfrak{z}\left(\widehat{\mathfrak{g l}}_{n}\right)=\left\{v \in V_{-n}\left(\mathfrak{g l}_{n}\right) \mid \mathfrak{g l}_{n}[t] v=0\right\}=\left\{v \in V_{-n}\left(\mathfrak{g l}_{n}\right) \mid \mathfrak{s l}_{n}[t] v=0\right\} .
$$

Iremos trabalhar com a álgebra de Lie $\widehat{\mathfrak{g l}}_{n} \oplus \mathbb{C} \tau$ onde o elemento $\tau$ satisfaz as seguintes relações:

$$
[\tau, X[r]]=-r X[r-1] \quad, \quad[\tau, K]=0 .
$$


Para todo $r \in \mathbb{Z}$, combine os elementos $E_{i j}[r]$ na matriz $E[r]$ da seguinte maneira:

$$
E[r]=\sum_{i, j=1}^{n} e_{i j} \otimes E_{i j}[r] \in \operatorname{End} \mathbb{C}^{n} \otimes \mathrm{U}
$$

onde $e_{i j}$ formam a base canônica de End $\mathbb{C}^{n}$ e U é a álgebra envolvente universal de $\widehat{\mathfrak{g r}_{n}} \oplus \mathbb{C} \tau$.

Dado $a \in\{1, \ldots, m\}$, defina o seguinte elemento:

$$
E[r]_{a}=\sum_{i, j=1}^{n} 1^{\otimes(a-1)} \otimes e_{i j} \otimes 1^{\otimes(m-a)} \otimes E_{i j}[r]
$$

pertencente a álgebra $\left(\operatorname{End} \mathbb{C}^{n}\right)^{\otimes m} \otimes \mathrm{U}$.

Proposição 3.4.1. A matriz $\varepsilon=\tau+E[-1]=\left(\delta_{i j} \tau+E_{i j}[-1]\right)$ com as entradas em $\widehat{\mathfrak{g l}}_{n} \oplus \mathbb{C} \tau$ é uma matriz de Manin.

Demonstração. Ver lema 7.1.2. da página 108 de Molev [2018].

Defina os elementos $\phi_{m a}, \psi_{m a}, \theta_{m a} \in \mathrm{U}\left(t^{-1} \mathfrak{g l}_{n}\left[t^{-1}\right]\right)$ pelas expansões:

$$
\begin{aligned}
& \operatorname{tr}_{1, \ldots, m} A^{(m)}(\tau+E[-1])_{1} \cdots(\tau+E[-1])_{m}=\phi_{m 0} \tau^{m}+\phi_{m 1} \tau^{m-1}+\cdots+\phi_{m m}, \\
& \operatorname{tr}_{1, \ldots, m} H^{(m)}(\tau+E[-1])_{1} \cdots(\tau+E[-1])_{m}=\psi_{m 0} \tau^{m}+\psi_{m 1} \tau^{m-1}+\cdots+\psi_{m m}, \\
& \operatorname{tr}(\tau+E[-1])^{m}=\theta_{m 0} \tau^{m}+\theta_{m 1} \tau^{m-1}+\cdots+\theta_{m m} .
\end{aligned}
$$

O determinante-coluna de $\tau+E[-1]$ é dado por:

$$
\operatorname{cdet}(\tau+E[-1])=\sum_{\sigma \in S_{n}} \operatorname{sgn} \sigma(\tau+E[-1])_{\sigma(1) 1} \cdots(\tau+E[-1])_{\sigma(n) n}
$$

e expandindo isso para um polinômio em $\tau$ temos:

$$
\operatorname{cdet}(\tau+E[-1])=\tau^{n}+\phi_{1} \tau^{n-1}+\cdots+\phi_{n} .
$$

Assim, como $\tau+E[-1]$ é uma matriz de Manin, temos:

$$
\operatorname{tr}_{1, \ldots, m} A^{(m)}(\tau+E[-1])_{1} \cdots(\tau+E[-1])_{m}=\operatorname{cdet}(\tau+E[-1])
$$

e, consequentemente, $\phi_{n a}=\phi_{a}$ para todo $a \in\{1, \ldots, n\}$.

Do corolário (3.3.1) temos a expansão

$$
\operatorname{cdet}(u+\tau+E[-1])=\sum_{m=0}^{n} u^{n-m} \operatorname{tr}_{1, \ldots, m} A^{(m)}\left(\tau+E[-1]_{1}\right) \cdots\left(\tau+E[-1]_{m}\right)
$$

onde $u$ é uma variável. Substituindo $\tau$ por $u+\tau$ em (3.32), obtemos as seguintes relações:

$$
\phi_{m a}=\left(\begin{array}{c}
n-a \\
m-a
\end{array}\right) \phi_{a}
$$

para todos $a, m \in\{1, \ldots, n\}$, com $a \leq m$. Em particular, $\phi_{m}=\phi_{m m}$ para todo $m \in\{1, \ldots, n\}$.

Teorema 3.4.1. Os elementos $\phi_{m}, \psi_{m a}$ e $\theta_{m a}$ pertencem ao centro de Feigin-Frenkel $\mathfrak{z}\left(\widehat{\mathfrak{g l}_{n}}\right)$. Além disso, os conjuntos $\left\{\phi_{1}, \ldots, \phi_{n}\right\},\left\{\psi_{11}, \ldots, \psi_{n n}\right\}$ e $\left\{\theta_{11}, \ldots, \theta_{n n}\right\}$ são conjuntos completos de vetores de Segal-Sugawara para $\mathfrak{g l}_{n}$.

Demonstração. Ver Chervov e Molev [2009]. 
Observação 3.4.1. Note que para $X[r] \in U=U\left(\widehat{\mathfrak{g l}_{n}} \oplus \mathbb{C} \tau\right)$ temos:

$$
\tau X[r]=X[r] \tau+[\tau, X[r]]=X[r]-r X[r-1] .
$$

Em particular:

$$
\tau E_{i j}[-1]=E_{i j}[-1] \tau+E_{i j}[-2]
$$

Exemplo 3.4.1. $E m \mathfrak{g l}_{2}$ :

(i) Vamos calcular $\phi_{1} e \phi_{2}$.

$$
\begin{aligned}
\operatorname{cdet}(\tau+E[-1]) & =(\tau+E[-1])_{11}(\tau+E[-1])_{22}-(\tau+E[-1])_{21}(\tau+E[-1])_{12}= \\
& =\left(\tau+E_{11}[-1]\right)\left(\tau+E_{22}[-1]\right)-E_{21}[-1] E_{12}[-1]= \\
& =\tau^{2}+E_{11}[-1] \tau+\tau E_{22}[-1]+\left(E_{11}[-1] E_{22}[-1]-E_{21}[-1] E_{12}[-1]\right)= \\
& =\tau^{2}+\left(E_{11}[-1]+E_{22}[-1]\right) \tau+ \\
& +\left(E_{22}[-2]+E_{11}[-1] E_{22}[-1]-E_{21}[-1] E_{12}[-1]\right)
\end{aligned}
$$

Então:

$$
\begin{aligned}
& \phi_{1}=E_{11}[-1]+E_{22}[-1] \\
& \phi_{2}=E_{22}[-2]+E_{11}[-1] E_{22}[-1]-E_{21}[-1] E_{12}[-1]
\end{aligned}
$$

(ii) Vamos calcular $\theta_{11}$ e $\theta_{22}$.

Para $m=1$ temos:

$$
\operatorname{tr}(\tau+E[-1])=\operatorname{tr}(I d) \tau+\operatorname{tr} E[-1]
$$

Então:

$$
\theta_{11}=\operatorname{tr} E[-1]
$$

Para $m=2$ temos:

$$
\begin{aligned}
\operatorname{tr}(\tau+E[-1])^{2} & =\operatorname{tr}\left(\tau^{2}+E[-1] \tau+\tau E[-1]+E[-1]^{2}\right)= \\
& =\operatorname{tr}\left(\tau^{2}+2 E[-1] \tau+E[-2]+E[-1]^{2}\right)= \\
& =\operatorname{tr}(I d) \tau^{2}+2 \operatorname{tr} E[-1] \tau+\left(\operatorname{tr} E[-2]+\operatorname{tr} E[-1]^{2}\right)
\end{aligned}
$$

Então:

$$
\theta_{22}=\operatorname{tr} E[-2]+\operatorname{tr} E[-1]^{2}
$$

(iii) Vamos calcular $\psi_{11}$ e $\psi_{22}$.

Para $m=1$ temos:

$$
\begin{aligned}
\operatorname{tr}_{1} H^{(1)}(\tau E[-1])_{1} & =\operatorname{tr} \sum_{1 \leq i \leq 2} \frac{1}{\alpha_{i} !} \sum_{\sigma \in S_{1}}(\tau+E[-1])_{i i}=\operatorname{tr}(\tau+E[-1])_{11}+\operatorname{tr}(\tau+E[-1])_{22}= \\
& =2 \tau+E_{11}[-1]+E_{22}[-1]
\end{aligned}
$$

Então:

$$
\psi_{11}=E_{11}[-1]+E_{22}[-1]
$$


Para $m=2$ temos:

$$
\begin{aligned}
& \operatorname{tr}_{1,2} H^{(2)}(\tau+E[-1])_{1}(\tau+E[-1])_{2}= \\
& =\sum_{\substack{\left(i_{1}, i_{2}\right)=(1,1),(1,2),(2,2)}} \frac{1}{\alpha_{1} ! \alpha_{2} !} \sum_{I d,(12)}(\tau+E[-1])_{i_{2} i_{\sigma(2)}}(\tau+E[-1])_{\left.i_{1} i_{\sigma(1)}\right)}= \\
& =\frac{1}{2}(\tau+E[-1])_{11}(\tau+E[-1])_{11}+\frac{1}{2}(\tau+E[-1])_{11}(\tau+E[-1])_{11}+ \\
& +(\tau+E[-1])_{22}(\tau+E[-1])_{11}+(\tau+E[-1])_{21}(\tau+E[-1])_{12}+ \\
& +\frac{1}{2}(\tau+E[-1])_{22}(\tau+E[-1])_{22}+\frac{1}{2}(\tau+E[-1])_{22}(\tau+E[-1])_{22}= \\
& =\left(\tau+E_{11}[-1]\right)\left(\tau+E_{11}[-1]\right)+\left(\tau+E_{22}[-1]\right)\left(\tau+E_{11}[-1]\right)+ \\
& +E_{21}[-1] E_{12}[-1]+\left(\tau+E_{22}[-1]\right)\left(\tau+E_{22}[-1]\right)= \\
& =\tau^{2}+2 E_{11}[-1] \tau+E_{22}[-2]+E_{11}[-1]^{2}+\tau^{2}+E_{22}[-1] \tau+ \\
& +E_{11}[-1] \tau+E_{11}[-2]+E_{22}[-1] E_{11}[-1]+E_{21}[-1] E_{12}[-1]+ \\
& +\tau^{2}+2 E_{22}[-1] \tau+E_{22}[-2]+E_{22}[-1]^{2}
\end{aligned}
$$

Então:

$$
\psi_{22}=2 E_{22}[-2]+E_{11}[-1]^{2}+E_{11}[-2]+E_{22}[-1] E_{11}[-1]+E_{21}[-1] E_{12}[-1]+E_{22}[-1]^{2}
$$

Exemplo 3.4.2. $E m \mathfrak{g l}_{3}$ :

(i) Vamos calcular $\phi_{1}, \phi_{2}$ e $\phi_{3}$.

$$
\begin{aligned}
& \operatorname{cdet}(\tau+E[-1])= \\
& =\left(\tau+E_{11}[-1]\right)\left(\tau+E_{22}[-1]\right)\left(\tau+E_{33}[-1]\right)-E_{21}[-1] E_{12}[-1]\left(\tau+E_{33}[-1]\right)- \\
& -E_{31}[-1]\left(\tau+E_{22}[-1]\right) E_{13}[-1]-\left(\tau+E_{11}[-1]\right) E_{32}[-1] E_{23}[-1]+ \\
& +E_{21}[-1] E_{32}[-1] E_{13}[-1]+E_{31}[-1] E_{12}[-1] E_{23}[-1]= \\
& =\tau^{3}+E_{11}[-1] \tau^{2}+\tau E_{22}[-1] \tau+\tau^{2} E_{33}[-1]+E_{11}[-1] E_{22}[-1] \tau+E_{11}[-1] \tau E_{33}[-1]+ \\
& +\tau E_{22}[-1] E_{33}[-1]-E_{21}[-1] E_{12}[-1] \tau-E_{31}[-1] \tau E_{13}[-1]-\tau E_{32}[-1] E_{23}[-1]+ \\
& +\left(E_{11}[-1] E_{22}[-1] E_{33}[-1]-E_{21}[-1] E_{12}[-1] E_{33}[-1]-E_{31}[-1] E_{22}[-1] E_{13}[-1]-\right. \\
& \left.-E_{11}[-1] E_{32}[-1] E_{23}[-1]+E_{21}[-1] E_{32}[-1] E_{13}[-1]+E_{31}[-1] E_{12}[-1] E_{23}[-1]\right)= \\
& =\tau^{3}+\left(E_{11}[-1]+E_{22}[-1]+E_{33}[-1]\right) \tau^{2}+ \\
& +\left(E_{22}[-2]+2 E_{33}[-2]+E_{11}[-1] E_{22}[-1]+E_{11}[-1] E_{33}[-1]+E_{22}[-1] E_{33}[-1]-\right. \\
& \left.-E_{21}[-1] E_{12}[-1]-E_{31}[-1] E_{13}[-1]-E_{32}[-1] E_{23}[-1]\right) \tau+ \\
& +\left(2 E_{33}[-1]+E_{11}[-1] E_{33}[-2]+E_{22}[-2] E_{33}[-1]+E_{22}[-1] E_{33}[-2]-\right. \\
& -E_{31}[-1] E_{13}[-2]-E_{32}[-2] E_{23}[-1]-E_{32}[-1] E_{23}[-2]+ \\
& +E_{11}[-1] E_{22}[-1] E_{33}[-1]-E_{21}[-1] E_{12}[-1] E_{33}[-1]-E_{31}[-1] E_{22}[-1] E_{13}[-1]- \\
& \left.-E_{11}[-1] E_{32}[-1] E_{23}[-1]+E_{21}[-1] E_{32}[-1] E_{13}[-1]+E_{31}[-1] E_{12}[-1] E_{23}[-1]\right)
\end{aligned}
$$


Então:

$$
\begin{aligned}
\phi_{1} & =E_{11}[-1]+E_{22}[-1]+E_{33}[-1] \\
\phi_{2} & =E_{22}[-2]+2 E_{33}[-2]+E_{11}[-1] E_{22}[-1]+E_{11}[-1] E_{33}[-1]+E_{22}[-1] E_{33}[-1]- \\
& -E_{21}[-1] E_{12}[-1]-E_{31}[-1] E_{13}[-1]-E_{32}[-1] E_{23}[-1] \\
\phi_{3} & =2 E_{33}[-1]+E_{11}[-1] E_{33}[-2]+E_{22}[-2] E_{33}[-1]+E_{22}[-1] E_{33}[-2]- \\
& -E_{31}[-1] E_{13}[-2]-E_{32}[-2] E_{23}[-1]-E_{32}[-1] E_{23}[-2]+ \\
& +E_{11}[-1] E_{22}[-1] E_{33}[-1]-E_{21}[-1] E_{12}[-1] E_{33}[-1]-E_{31}[-1] E_{22}[-1] E_{13}[-1]- \\
& -E_{11}[-1] E_{32}[-1] E_{23}[-1]+E_{21}[-1] E_{32}[-1] E_{13}[-1]+E_{31}[-1] E_{12}[-1] E_{23}[-1]
\end{aligned}
$$

(ii) Vamos calcular $\theta_{11}, \theta_{22}$ e $\theta_{33}$.

Note que as contas para $\theta_{m a}$ não dependem de $n$. Então $\theta_{11}$ e $\theta_{22}$ são iguais aos calculados para $\mathfrak{g l}_{2}$.

$$
\begin{aligned}
& \theta_{11}=\operatorname{tr} E[-1] \\
& \theta_{22}=\operatorname{tr} E[-2]+\operatorname{tr} E[-1]^{2}
\end{aligned}
$$

Assim, só temos que calcular $\theta_{33}$. Para $m=3$ temos:

$$
\begin{aligned}
& \operatorname{tr}(\tau+E[-1])^{3}= \\
& =\operatorname{tr}\left(\tau^{3}+\tau E[-1] \tau+E[-1] \tau^{2}+E[-1]^{2} \tau+\tau^{2} E[-1]+\tau E[-1]^{2}+E[-1] \tau E[-1]+E[-1]^{3}\right)= \\
& =\operatorname{tr}\left(\tau^{3}+E[-1] \tau^{2}+E[-2] \tau+E[-1] \tau^{2}+E[-1]^{2} \tau+E[-1] \tau^{2}+2 E[-2] \tau+\right. \\
& \left.+2 E[-3]+E[-1]^{2} \tau+E[-1] E[-2]+E[-2] E[-1]+E[-1]^{2} \tau+E[-1] E[-2]+E[-1]^{3}\right)= \\
& =3 \tau^{3}+3 \operatorname{tr} E[-1] \tau^{2}+\left(3 \operatorname{tr} E[-2]+3 \operatorname{tr} E[-1]^{2}\right) \tau+ \\
& +\left(2 \operatorname{tr} E[-3]+2 \operatorname{tr} E[-1] E[-2]+\operatorname{tr} E[-2] E[-1]+\operatorname{tr} E[-1]^{3}\right)
\end{aligned}
$$

Então:

$$
\theta_{33}=2 \operatorname{tr} E[-3]+2 \operatorname{tr} E[-1] E[-2]+\operatorname{tr} E[-2] E[-1]+\operatorname{tr} E[-1]^{3}
$$

\subsubsection{Geradores de $A_{\mu}$}

Aplicando o homomorfismo $\varrho_{\mu, z}$ de (3.12) nos elementos de $\mathfrak{z}\left(\widehat{\mathfrak{g l}_{n}}\right)$ do teorema 3.4.1 e fazendo a expansão dos resultados como em (3.13), obtemos elementos de $A_{\mu} \subset \mathrm{U}\left(\mathfrak{g l}_{n}\right)$. Para dar formas explícitas para esses elementos, usaremos a álgebra tensorial $\left(\text { End } \mathbb{C}^{n}\right)^{\otimes m} \otimes \mathrm{U}$, onde U agora denotará a álgebra de operadores diferenciais cujos elementos são somas finitas com a seguinte forma:

$$
\sum_{k, l \geq 0} u_{k l} z^{-k} \partial_{z}^{l} u_{k l} \in \mathrm{U}\left(\mathfrak{g l}_{n}\right)
$$

$\operatorname{com} u_{k l} \in \mathrm{U}\left(\mathfrak{g l}_{n}\right)$.

Podemos estender o homomorfismo $\varrho_{\mu, z}$ de (3.12) para $\mathfrak{g}=\widehat{\mathfrak{g l}}_{n} \oplus \mathbb{C} \tau$, sendo $\partial_{z}$ a imagem do elemento $-\tau$.

Como na subseção anterior, temos:

$$
E=\sum_{i, j=1}^{n} e_{i j} \otimes E_{i j} \in \operatorname{End} \mathbb{C}^{n} \otimes \mathrm{U}\left(\mathfrak{g l}_{n}\right) .
$$

Defina os polinômios (dependentes de $\mu) \phi_{m a}(z), \psi_{m a}(z)$ e $\theta_{m a}(z)$ em $z^{-1}$ com coeficientes em 
$\mathrm{U}\left(\mathfrak{g l}_{n}\right)$ pelas expansões:

$$
\begin{aligned}
& \operatorname{tr}_{1, \ldots, m} A^{(m)} M_{1} \ldots M_{m}=\phi_{m 0}(z) \partial_{z}^{m}+\phi_{m 1}(z) \partial_{z}^{m-1}+\cdots+\phi_{m m}(z) \\
& \operatorname{tr}_{1, \ldots, m} H^{(m)} M_{1} \ldots M_{m}=\psi_{m 0}(z) \partial_{z}^{m}+\psi_{m 1}(z) \partial_{z}^{m-1}+\cdots+\psi_{m m}(z) \\
& \operatorname{tr} M^{m}=\theta_{m 0}(z) \partial_{z}^{m}+\theta_{m 1}(z) \partial_{z}^{m-1}+\cdots+\theta_{m m}(z)
\end{aligned}
$$

onde $M=-\partial_{z}+\mu+E z^{-1}=\left(-\delta_{i j} \partial_{z}+\mu\left(E_{i j}\right)+E_{i j} z^{-1}\right)$.

Além disso, analogamente ao que fizemos na seção anterior, definimos polinômios $\phi_{a}(z)$ pela expansão do determinante coluna

$$
\operatorname{cdet} M=\phi_{0}(z) \partial_{z}^{m}+\phi_{1}(z) \partial_{z}^{m-1}+\cdots+\phi_{n}(z) .
$$

Também temos:

$$
\phi_{m a}(z)=\left(\begin{array}{c}
n-a \\
m-a
\end{array}\right) \phi_{a}(z)
$$

para todo $a, m \in\{1, \ldots, n\}$ com $a \leq m$, e $\phi_{m m}=\phi_{m}(z)=\phi_{n m}$ para todo $m \in\{1, \ldots, n\}$.

Dando uma notação para os coeficientes dos polinômios $\phi_{m}(z), \psi_{m m}(z)$ e $\theta_{m m}(z)$ obtemos:

$$
\begin{aligned}
& \phi_{m}(z)=\phi_{m}^{(0)} z^{-m}+\cdots+\phi_{m}^{(m-1)} z^{-1}+\phi_{m}^{(m)}, \\
& \psi_{m}(z)=\psi_{m}^{(0)} z^{-m}+\cdots+\psi_{m}^{(m-1)} z^{-1}+\psi_{m}^{(m)}, \\
& \theta_{m}(z)=\theta_{m}^{(0)} z^{-m}+\cdots+\theta_{m}^{(m-1)} z^{-1}+\theta_{m}^{(m)} .
\end{aligned}
$$

Teorema 3.4.2. Dado $\mu \in \mathfrak{g l}_{n}$, todos os coeficientes dos polinômios $\phi_{m}(z), \psi_{m a}(z)$ e $\theta_{m a}(z)$ pertencem à subálgebra comutativa $A_{\mu}$ de $U\left(\mathfrak{g l}_{n}\right)$. Além disso, os conjuntos $\left\{\phi_{m}^{(k)} \mid 0 \leq k<m \leq n\right\}$, $\left\{\psi_{m m}^{(k)} \mid 0 \leq k<m \leq n\right\}$ e $\left\{\theta_{m m}^{(k)} \mid 0 \leq k<m \leq n\right\}$ são conjuntos geradores de $A_{\mu}$. Se $\mu$ é regular, esses conjuntos são formados por elementos algebricamente independentes.

Demonstração. É consequência do teorema 3.4.1 e de resultados de Feigin et al. [2010] e de Rybnikov [2006].

Observação 3.4.2. Como $-\partial_{z}$ é imagem de $\tau$ por $\varrho_{\mu, z}$, pela observação 3.4 .1 temos:

$$
-\partial_{z} X z^{r}=-X z^{r} \partial_{z}-r X z^{r-1}-r \delta_{r-1,-1} \mu(X)
$$

para todo $X \in \mathfrak{g l}_{n}$. Em particular:

$$
-\partial_{z} E_{i j} z^{-1}=-E_{i j} z^{-1} \partial_{z}+E_{i j} z^{-2}
$$

Além disso, $\mu$ comuta com $\partial_{z}$ e com $X z^{r}$, para todo $X \in \mathfrak{g l}_{n}$.

Exemplo 3.4.3. $E m \mathfrak{g l}_{2}$ :

(i) Vamos calcular $\phi_{1}^{(0)}, \phi_{2}^{(0)}$ e $\phi_{2}^{(1)}$.

$$
\begin{aligned}
& \operatorname{cdet}\left(-\partial_{z}+\mu+E z^{-1}\right)= \\
& =\left(-\partial_{z}+\mu+E z^{-1}\right)_{11}\left(-\partial_{z}+\mu+E z^{-1}\right)_{22}-\left(-\partial_{z}+\mu+E z^{-1}\right)_{21}\left(-\partial_{z}+\mu+E z^{-1}\right)_{12} \\
& =\left(-\partial_{z}+\mu\left(E_{11}\right)+E_{11} z^{-1}\right)\left(-\partial_{z}+\mu\left(E_{22}\right)+E_{22} z^{-1}\right)-\left(\mu\left(E_{21}\right)+E_{21} z^{-1}\right)\left(\mu\left(E_{12}\right)+E_{12} z^{-1}\right)= \\
& =\partial_{z}^{2}+\left(\mu\left(E_{11}\right)+E_{11} z^{-1}+\mu\left(E_{22}\right)+E_{22} z^{-1}\right) \partial_{z}+ \\
& +\left(E_{22} z^{-2}+\left(\mu\left(E_{11}\right)+E_{11} z^{-1}\right)\left(\mu\left(E_{22}\right)+E_{22} z^{-1}\right)-\left(\mu\left(E_{21}\right)+E_{21} z^{-1}\right)\left(\mu\left(E_{12}\right)+E_{12} z^{-1}\right)\right)
\end{aligned}
$$

Assim, temos:

$$
\begin{aligned}
& \phi_{1}=\mu\left(E_{11}\right)+E_{11} z^{-1}+\mu\left(E_{22}\right)+E_{22} z^{-1} \\
& \phi_{2}=E_{22} z^{-2}+\left(\mu\left(E_{11}\right)+E_{11} z^{-1}\right)\left(\mu\left(E_{22}\right)+E_{22} z^{-1}\right)-\left(\mu\left(E_{21}\right)+E_{21} z^{-1}\right)\left(\mu\left(E_{12}\right)+E_{12} z^{-1}\right)
\end{aligned}
$$


Então:

$$
\begin{aligned}
& \phi_{1}^{(0)}=E_{11}+E_{22} \\
& \phi_{2}^{(0)}=E_{22}+E_{11} E_{22}+E_{21} E_{12} \\
& \phi_{2}^{(0)}=E_{11} \mu\left(E_{22}\right)+\mu\left(E_{11}\right) E_{22}-E_{21} \mu\left(E_{12}\right)-\mu\left(E_{21}\right) E_{12} .
\end{aligned}
$$

Poderiamos ter também aplicado o homomorfismo $\varrho_{\mu, z}$ nos $\phi_{1}$ e $\phi_{2}$ obtidos no exemplo 3.4.1 e feito a expansão (3.13), assim teriamos:

$$
\begin{aligned}
\varrho_{\mu, z}\left(\phi_{1}\right) & =\varrho_{\mu, z}\left(E_{11}[-1]+E_{22}[-1]\right)=E_{11} z^{-1}+\mu\left(E_{11}\right)+E_{22} z^{-1}+\mu\left(E_{22}\right)= \\
& =\left(E_{11}+E_{22}\right) z^{-1}+\left(\mu\left(E_{11}\right)+\mu\left(E_{22}\right)\right)
\end{aligned}
$$

$e$

$$
\begin{aligned}
& \varrho_{\mu, z}\left(\phi_{2}\right)=\varrho_{\mu, z}\left(E_{22}[-2]+E_{11}[-1] E_{22}[-1]-E_{21}[-1] E_{12}[-1]\right)= \\
& =E_{22} z^{-2}+\left(E_{11} z^{-1}+\mu\left(E_{11}\right)+\left(E_{22} z^{-1}+\mu\left(E_{22}\right)\right)-\left(E_{21} z^{-1}+\mu\left(E_{21}\right)\right)\left(E_{12} z^{-1}+\mu\left(E_{12}\right)\right)=\right. \\
& =\left(E_{22}+E_{11} E_{22}-E_{21} E_{12}\right) z^{-2}+\left(E_{11} \mu\left(E_{22}\right)+\mu\left(E_{11}\right) E_{22}-E_{21} \mu\left(E_{12}\right)-\mu\left(E_{21}\right) E_{12}\right) z^{-1}+ \\
& +\left(\mu\left(E_{11}\right) \mu\left(E_{22}\right)-\mu\left(E_{21}\right) \mu\left(E_{12}\right)\right)
\end{aligned}
$$

obtendo o mesmo resultado anterior.

(ii) Vamos calcular $\theta_{1}^{(0)}, \theta_{2}^{(0)}$ e $\theta_{2}^{(1)}$.

Para $m=1$ temos:

$$
\operatorname{tr}\left(-\partial_{z}+\mu+E z^{-1}\right)=-\operatorname{tr}(I d) \partial_{z}+\operatorname{tr} \mu+\operatorname{tr} E z^{-1}
$$

Então:

$$
\theta_{11}(z)=\operatorname{tr} \mu+\operatorname{tr} E z^{-1}
$$

O que implica que:

$$
\theta_{11}^{(0)}=\operatorname{tr} E
$$

Para $m=2$ temos:

$$
\begin{aligned}
& \operatorname{tr}\left(-\partial_{z}+\mu+E z^{-1}\right)^{2}=\operatorname{tr}\left(\partial_{z}^{2}-2 \mu \partial_{z}-2 E z^{-1} \partial_{z}+\mu^{2}+2 \mu E z^{-1}+E^{2} z^{-2}+E z^{-2}\right)= \\
& \quad=\operatorname{tr}(I d) \partial_{z}^{2}+\operatorname{tr}\left(-2 \mu-2 E z^{-1}\right) \partial_{z}+\operatorname{tr}\left(\mu^{2}+2 \mu E z^{-1}+E^{2} z^{-2}+E z^{-2}\right)
\end{aligned}
$$

Então:

$$
\theta_{22}(z)=\left(\operatorname{tr} E^{2}+\operatorname{tr} E\right) z^{-2}+2 \operatorname{tr} \mu E z^{-1}+\operatorname{tr} \mu^{2}
$$

O que implica que:

$$
\begin{aligned}
& \theta_{22}(0)=\operatorname{tr} E^{2}+\operatorname{tr} E \\
& \theta_{22}(1)=2 \operatorname{tr} \mu E
\end{aligned}
$$

Exemplo 3.4.4. $E m \mathfrak{g l}_{3}$ :

Vamos calcular $\theta_{11}^{(0)}, \theta_{22}^{(0)}, \theta_{22}^{(1)}, \theta_{33}^{(0)}, \theta_{33}^{(1)}$ e $\theta_{33}^{(2)}$. Note que para calcular $\theta_{m m}^{(k)}$ não usamos $n$, então 
$\theta_{11}^{(0)}, \theta_{22}^{(0)}$ e $\theta_{22}^{(1)}$ para $\mathfrak{g l}_{3}$ são os mesmos de $\mathfrak{g l}_{2}$ :

$$
\begin{aligned}
& \theta_{11}^{(0)}=\operatorname{tr} E \\
& \theta_{22}(0)=\operatorname{tr} E^{2}+\operatorname{tr} E \\
& \theta_{22}(1)=2 \operatorname{tr} \mu E
\end{aligned}
$$

Falta calcular $\theta_{33}^{(0)}, \theta_{33}^{(1)}$ e $\theta_{33}^{(2)}$.

Aplicando $\varrho_{\mu, z}$ em $\theta_{33}$ do exemplo 3.4.2, temos:

$$
\begin{aligned}
& \varrho_{\mu, z}\left(\theta_{33}\right)=\varrho_{\mu, z}\left(2 \operatorname{tr} E[-3]+2 \operatorname{tr} E[-1] E[-2]+\operatorname{tr} E[-2] E[-1]+\operatorname{tr} E[-1]^{3}\right)= \\
& =2 \operatorname{tr} E z^{-3}+2 \operatorname{tr} E^{2} z^{-3}+2 \operatorname{tr} \mu E z^{-2}+\operatorname{tr} E^{2} z^{-3}+\operatorname{tr} \mu E z^{-2}+\operatorname{tr} E^{3} z^{-3}+ \\
& +3 \operatorname{tr} \mu E^{2} z^{-2}+3 \operatorname{tr} \mu^{2} E z^{-1}+\operatorname{tr} \mu^{3}
\end{aligned}
$$

pois, por exemplo, temos:

$$
\begin{aligned}
\operatorname{tr} E[-1] E[-2] & =\operatorname{tr}\left(\sum_{i, j=1}^{2} e_{i j} \otimes E_{i j}[-1]\right)\left(\sum_{k, l=1}^{2} e_{k l} \otimes E_{k l}[-2]\right)= \\
& =\operatorname{tr}\left(\sum_{i, j, k, l=1}^{2} e_{i j} e_{k l} \otimes E_{i j}[-1] E_{k l}[-2]\right)= \\
& =\operatorname{tr}\left(\sum_{i, j, l=1}^{2} e_{i l} \otimes E_{i j}[-1] E_{j l}[-2]\right)= \\
& =\left(\sum_{i, j=1}^{2} E_{i j}[-1] E_{j i}[-2]\right)
\end{aligned}
$$

$e$

$$
\varrho_{\mu, z}(\operatorname{tr} E[-1] E[-2])=\left(\sum_{i, j=1}^{2} E_{i j} E_{j i} z^{-3}\right)+\left(\sum_{i, j=1}^{2} \mu\left(E_{i j}\right) E_{j i} z^{-2}\right)=\operatorname{tr} E^{2} z^{-3}+\operatorname{tr} \mu E z^{-2}
$$

Assim concuimos que:

$$
\begin{aligned}
\theta_{33}^{(0)} & =2 \operatorname{tr} E+3 \operatorname{tr} E^{2}+\operatorname{tr} E^{3} \\
\theta_{33}^{(1)} & =3 \operatorname{tr} \mu E+3 \operatorname{tr} \mu E^{2} \\
\theta_{33}^{(2)} & =3 \operatorname{tr} \mu^{2} E
\end{aligned}
$$

\subsubsection{Geradores de $A_{\mu}$ algebricamente independentes}

Dada uma forma bilinear simétrica não degenerada $B$ de $\mathfrak{g l}_{n}$, temos que $\mathfrak{g l}_{n} \cong \mathfrak{g l}_{n}^{*}$ através do isomorfismo $f$ que leva $x \in \mathfrak{g l}_{n}$ em $f(x) \in \mathfrak{g l}_{n}^{*}$, onde $f(x)$ é o funcional linear que leva $y \in \mathfrak{g l}_{n} \mathrm{em}$ $B(x, y)$. Dessa forma, veremos todos $\mu \in \mathfrak{g l}_{n}^{*}$ como matrizes $n \times n$.

Seja $\mu \in \mathfrak{g l}_{n}$. Suponha que os diferentes autovalores de $\mu$ são $\lambda_{1}, \ldots, \lambda_{r}$ e que a forma de Jordan de $\mu$ é dada pelos blocos de Jordan $J_{\alpha_{j}^{(i)}}\left(\lambda_{i}\right)$ de tamanhos $\alpha_{1}^{(i)} \geq \alpha_{2}^{(i)} \geq \ldots \geq \alpha_{s_{i}}^{(i)} \geq 1$. Denote por $\alpha^{(i)}$ o diagrama de Young cuja $j$-ésima linha possui $\alpha_{j}^{(i)}$ caixas (quadrados) e denote por $\left|\alpha^{(i)}\right|$ o número total de caixas em $\alpha^{(i)}$. 
Defina agora outro diagrama de Young $\gamma$ tal que sua $l$-ésima linha tem $\gamma_{l}$ caixas, onde:

$$
\gamma_{l}=\sum_{i=1}^{r} \sum_{j \geq l+1} \alpha_{j}^{(i)}
$$

ou seja, $\gamma_{l}$ é a soma do número de caixas que estão abaixo da $l$-ésima linha de todos diagramas $\alpha^{(i)}$.

Seja $\Gamma$ o diagrama de Young cuja $k$-ésima linha tem $n-k+1$ caixas. Preencha $\Gamma$ com os geradores $\phi_{m}^{(k)}$ de $A_{\mu}$ de forma que a $j$-ésima caixa da $i$-ésima linha tenha o gerador $\phi_{n-j+1}^{(n-i-j+1)}$. Assim temos:

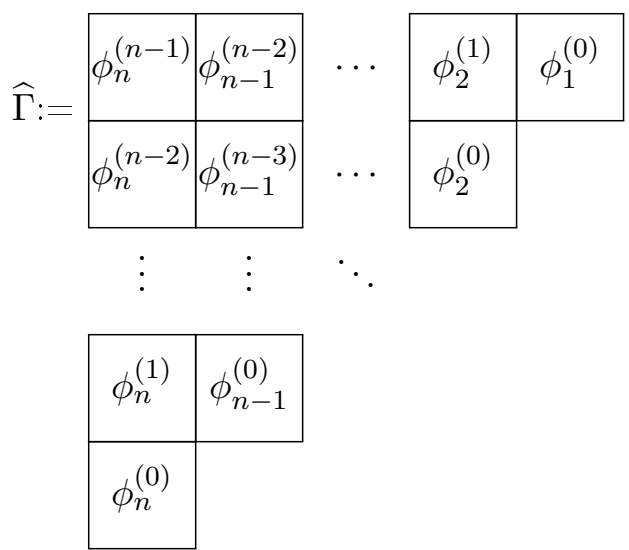

Note que o diagrama $\gamma$ está contido em $\Gamma$, ou seja, a quantidade de linhas de $\gamma$ é menor ou igual à quantidade de linhas de $\Gamma$ e a quantidade de caixas na $i$-ésima linha de $\gamma$ é menor ou igual à quantidade de caixas da $i$-ésima linha de $\Gamma$. Assim podemos definir o diagrama $\Gamma / \gamma$ que é $\Gamma$ sem as caixas que também estão presentes em $\gamma$. Da mesma forma que preenchemos $\Gamma$ podemos preencher $\Gamma / \gamma$, ou seja, na $j$-ésima caixa da $i$-ésima linha colocamos o gerador $\phi_{n-j+1}^{n-i-j+1}$. Denote esse diagrama preenchido por $\widehat{\Gamma} / \gamma$.

Teorema 3.4.3. Os elementos $\phi_{m}^{(k)}$ que aparecem em $\widehat{\Gamma} / \gamma$ são geradores algebricamente independentes da subálgebra $A_{\mu}$. Além disso, a subálgebra $A_{\mu}$ é a quantização de $\overline{A_{\mu}}$, ou seja, gr $A_{\mu}=\overline{A_{\mu}}$.

Demonstração. Esse é o teorema principal de Futorny e Molev [2015].

Exemplo 3.4.5. Seja $\mu$ regular. Então $\mu$ só possui um bloco de Jordan para cada autovalor, ou seja, os diagramas $\alpha^{i}$ só têm uma linha. Assim o diagrama $\gamma$ não possui nenhuma linha, é vázio. Portanto todos geradores $\phi_{m}^{(k)}$ que aparecem em $\widehat{\Gamma}$ são algebricamente independentes.

Exemplo 3.4.6. Seja $\mu$ uma matrix escalar, ou seja, $\mu$ tem $n$ blocos de Jordan de tamanho 1 para seu único autovalor. Assim, $\gamma$ tem $n-j$ caixas na sua $j$-ésima linha, $j \in\{1, \ldots, n-1\}$. Portanto:

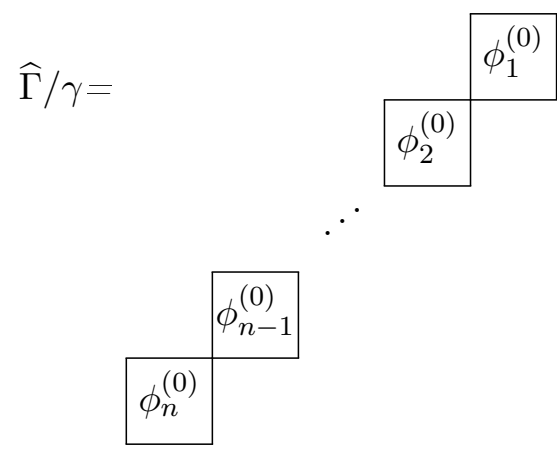

$e \phi_{1}^{(0)}, \ldots, \phi_{n}^{(0)}$ são geradores algebricamente independentes. 
Exemplo 3.4.7. Seja $\mu$ a matriz abaixo:

$$
\left(\begin{array}{ccccccc}
\lambda_{1} & 0 & 0 & 0 & 0 & 0 & 0 \\
0 & \lambda_{1} & 0 & 0 & 0 & 0 & 0 \\
0 & 0 & \lambda_{1} & 0 & 0 & 0 & \\
0 & 0 & 0 & \lambda_{2} & 1 & 0 & 0 \\
0 & 0 & 0 & 0 & \lambda_{2} & 0 & 0 \\
0 & 0 & 0 & 0 & 0 & \lambda_{3} & 0 \\
0 & 0 & 0 & 0 & 0 & 0 & \lambda_{3}
\end{array}\right)
$$

onde $\lambda_{1}, \lambda_{2}$ e $\lambda_{3}$ são distintos. Então temos para $\lambda_{1}$ três blocos de Jordan de tamanho 1, para $\lambda_{2}$ um bloco de Jordan de tamanho 2 e para para $\lambda_{3}$ dois blocos de Jordan de tamanho 1. Assim:

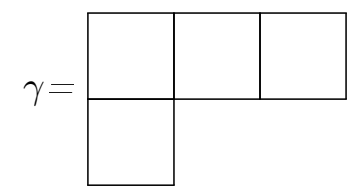

$e$

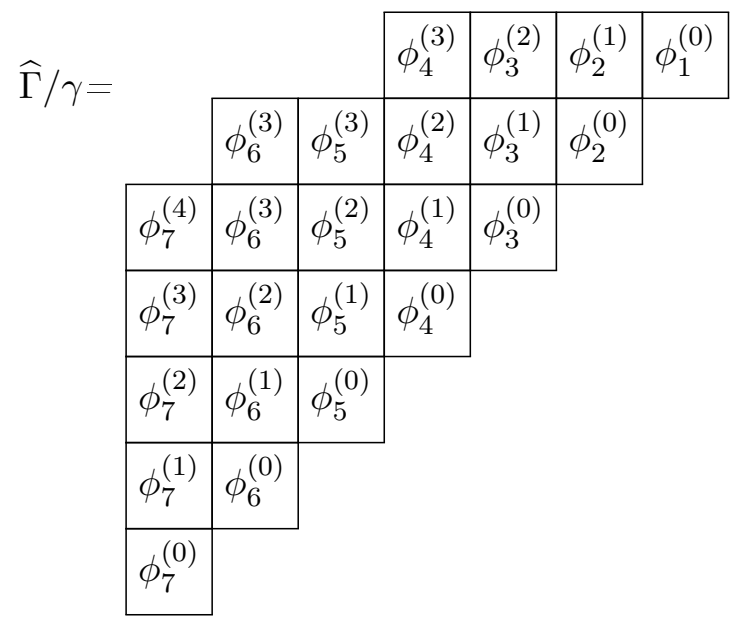

Portanto, os elementos da forma $\phi_{m}^{(k)}$ que aparecem no diagrama acima são geradores algebricamente independente de $A_{\mu}$.

\subsection{Tipo B e D}

Nessa seção vamos focar o estudo para as álgebras de Lie de tipo $B$ e $D$.

Seja $\mathfrak{g}=\mathfrak{o}_{N}, \operatorname{com} N=2 n+1$ (tipo $B, n \geq 1$ ) ou $N=2 n$ (tipo $D, n \geq 3$ ), a álgebra de Lie ortogonal. $\mathfrak{o}_{N}$ pode ser vista como uma subálgebra de $\mathfrak{g l}_{N}$, gerada pelos elementos $F_{i j}:=E_{i j}-E_{j^{\prime} i^{\prime}}$, onde $i^{\prime}=N-i+1$. Note que $F_{i j}=-F_{j^{\prime} i^{\prime}}$.

\subsubsection{Geradores do centro de Feigin-Frenkel}

O número de Coxeter dual para a álgebra de Lie ortogonal é $h^{\vee}=N-2$. Assim, a forma de Killing normalizada é dada por

$$
\langle X, Y\rangle=\frac{1}{2} \operatorname{tr} X Y
$$

para todos $X, Y \in \mathfrak{o}_{N}$. 
Considere a álgebra de Kac-Moody afim $\widehat{\mathfrak{o}_{N}}=\mathfrak{o}_{N}\left[t, t^{-1}\right] \oplus \mathbb{C} K$. Denote por $F_{i j}[r]$ os elementos $F_{i j} t^{r}$, para todo $r \in \mathbb{Z}$. O colchete de $\widehat{\mathfrak{o}_{N}}$ é definido como:

$$
\begin{aligned}
{\left[F_{i j}[r], F_{k l}[s]\right]=} & \delta_{k j} F_{i l}[r+s]-\delta_{i l} F_{k j}[r+s]-\delta_{k i^{\prime}} F_{j^{\prime} l}[r+s]+\delta_{j^{\prime} l} F_{k i^{\prime}}[r+s]+ \\
& +r \delta_{r,-s} K\left(\delta_{k j} \delta_{i l}-\delta_{k i^{\prime}} \delta_{j^{\prime} l}\right)
\end{aligned}
$$

onde $K$ é um elemento central.

Para todo $r \in \mathbb{Z}$ defina o seguinte elemento:

$$
F[r]=\sum_{i, j=1}^{N} e_{i j} \otimes F_{i j}[r]
$$

pertencente a End $\mathbb{C}^{n} \otimes \mathrm{U}\left(\widehat{\mathfrak{o}_{n}}\right)$.

Para $a, m \in\{1, \ldots, N\}$, com $a \leq m$, defina o elemento:

$$
F[r]_{a}=\sum_{i, j=1}^{N} 1^{\otimes(a-1)} \otimes e_{i j} \otimes 1^{\otimes(m-a)} \otimes F_{i j}[r]
$$

pertencente a $\left(\text { End } \mathbb{C}^{n}\right)^{\otimes m} \otimes \mathrm{U}\left(\widehat{\mathfrak{o}_{n}}\right)$.

Para $N=2 n$ par, também definiremos o elemento Pfaffian:

$$
\operatorname{Pf} F[-1]=\frac{1}{2^{n} n !} \sum_{\sigma \in S_{2 n}} \operatorname{sgn} \sigma \cdot \prod_{i=1}^{n} F_{\sigma(2 i-1) \sigma(2 i)^{\prime}}[-1]
$$

pertencente a álgebra envolvente universal $\mathrm{U}\left(t^{-1} \mathfrak{o}_{N}\left[t^{-1}\right]\right)$.

Observação 3.5.1. Em álgeba linear, dada uma matriz $A=\left(a_{i j}\right)$ antissimétrica (i.e. $A=-A^{t}$, onde $A^{t}$ é a transposta de A) com entradas em um corpo $k$ fechado de característica 0 , seu determinante pode ser escrito como o quadrado de um polinômio nas entradas das matrizes com coeficientes inteiros. Ou seja, existe $p \in k\left[a_{i j}: 1 \leq i, j \leq n\right]$ tal que detA $=p^{2}$. Esse polinômio p recebe o nome de Pfaffian de A. O Pfaffian de A é dado por:

$$
\operatorname{PfF}[-1]=\frac{1}{2^{n} n !} \sum_{\sigma \in S_{2 n}} \operatorname{sgn} \sigma \cdot \prod_{i=1}^{n} a_{\sigma(2 i-1) \sigma(2 i)}
$$

Assim, o elemento Pfaffian definido acima é uma definição análoga a usada em álgebra linear mas para as matrizes de End $\mathbb{C}^{n} \otimes U\left(\widehat{\mathfrak{o}_{n}}\right)$.

Exemplo 3.5.1. Vamos calcular PfF[-1] para $n=2$.

Temos:

$$
\begin{aligned}
S_{4}= & \{1,(12),(13),(14),(23),(24),(34),(13)(12),(14)(12),(12)(13),(14)(13),(12)(14),(13)(14), \\
& (24)(23),(23)(24),(12)(34),(13)(24),(14)(23),(14)(13)(12),(13)(14)(12),(14)(12)(13), \\
& (12)(14)(13),(13)(12)(14),(12)(13)(14)\}
\end{aligned}
$$




$$
\begin{aligned}
\operatorname{PfF}[-1] & =\frac{1}{2^{2} 2 !} \sum_{\sigma \in S_{4}} s g n \sigma \cdot F_{\sigma(1) \sigma(2)^{\prime}}[-1] F_{\sigma(3) \sigma(4)^{\prime}}[-1]= \\
& =\frac{1}{8}\left(F_{13}[-1] F_{31}[-1]-F_{24}[-1] F_{31}[-1]-F_{33}[-1] F_{11}[-1]-F_{43}[-1] F_{34}[-1]-\right. \\
& -F_{12}[-1] F_{21}[-1]-F_{11}[-1] F_{33}[-1]-F_{13}[-1] F_{42}[-1]+F_{22}[-1] F_{11}[-1]+ \\
& +F_{21}[-1] F_{34}[-1]+F_{34}[-1] F_{21}[-1]+F_{33}[-1] F_{44}[-1]+F_{44}[-1] F_{33}[-1]+ \\
& +F_{43}[-1] F_{12}[-1]+F_{12}[-1] F_{43}[-1]+F_{11}[-1] F_{22}[-1]+F_{24}[-1] F_{42}[-1]+ \\
& +F_{31}[-1] F_{13}[-1]+F_{42}[-1] F_{24}[-1]-F_{22}[-1] F_{44}[-1]-F_{21}[-1] F_{12}[-1]- \\
& \left.-F_{31}[-1] F_{24}[-1]-F_{34}[-1] F_{43}[-1]-F_{42}[-1] F_{13}[-1]-F_{44}[-1] F_{22}[-1]\right)= \\
& =\frac{1}{8}\left(F_{13}[-1] F_{31}[-1]-F_{24}[-1] F_{31}[-1]-F_{13}[-1] F_{42}[-1]+F_{24}[-1] F_{42}[-1]\right)+ \\
& +\frac{1}{8}\left(-F_{12}[-1] F_{21}[-1]+F_{34}[-1] F_{21}[-1]+F_{12}[-1] F_{43}[-1]-F_{34}[-1] F_{43}[-1]\right)+ \\
& +\frac{1}{8}\left(F_{11}[-1] F_{22}[-1]-F_{11}[-1] F_{33}[-1]-F_{44}[-1] F_{22}[-1]+F_{44}[-1] F_{33}[-1]\right)+ \\
& +\frac{1}{8}\left(F_{31}[-1] F_{13}[-1]-F_{31}[-1] F_{24}[-1]-F_{42}[-1] F_{13}[-1]+F_{42}[-1] F_{24}[-1]\right)+ \\
& +\frac{1}{8}\left(-F_{21}[-1] F_{12}[-1]+F_{21}[-1] F_{34}[-1]+F_{43}[-1] F_{12}[-1]-F_{43}[-1] F_{34}[-1]\right)+ \\
& +\frac{1}{8}\left(F_{22}[-1] F_{11}[-1]-F_{22}[-1] F_{44}[-1]-F_{33}[-1] F_{11}[-1]+F_{33}[-1] F_{44}[-1]\right)= \\
& =\frac{1}{2}\left(F_{13}[-1] F_{31}[-1]-F_{12}[-1] F_{21}[-1]+F_{11}[-1] F_{22}[-1]+F_{31}[-1] F_{13}[-1]-\right. \\
& \left.-F_{21}[-1] F_{12}[-1]+F_{22}[-1] F_{11}[-1]\right)
\end{aligned}
$$

Por (3.52), temos:

$$
\begin{aligned}
& F_{31}[-1] F_{13}[-1]=F_{13}[-1] F_{31}[-1]+F_{33}[-2]-F_{11}[-2] \\
& F_{21}[-1] F_{12}[-1]=F_{12}[-1] F_{21}[-1]+F_{22}[-2]-F_{11}[-2] \\
& F_{22}[-1] F_{11}[-1]=F_{11}[-1] F_{22}[-1]
\end{aligned}
$$

$e$

$$
F_{33}=-F_{3^{\prime} 3^{\prime}}=-F_{22} .
$$

Portanto:

$$
P f[-1]=F_{13}[-1] F_{31}[-1]-F_{12}[-1] F_{21}[-1]+F_{11}[-1] F_{22}[-1]-F_{22}[-2] .
$$

Proposição 3.5.1. O elemento PfF $[-1]$ pertence ao centro de Feigin-Frenkel $\mathfrak{z}\left(\widehat{o}_{2 n}\right)$.

Demonstração. Ver proposição 8.1.4. da página 122 de Molev [2018].

Iremos trabalhar com a álgebra de Lie estendida $\widehat{\mathfrak{a}_{N}} \oplus \mathbb{C} \tau$ onde o elemento $\tau$ satisfaz as seguintes relações:

$$
[\tau, X[r]]=-r X[r-1] \quad, \quad[\tau, K]=0 .
$$

Defina a seguinte constante

$$
\gamma_{m} N=\frac{N+m-2}{N+2 m-2}
$$

Considere o módulo vácuo no nível crítico $V_{-h^{\vee}}\left(\mathfrak{o}_{N}\right) \cong \mathrm{U}\left(t^{-1} \mathfrak{o}_{N}\left[t^{-1}\right]\right)$. Defina os elementos $\phi_{m a}$ 
de $V_{-h^{\vee}}\left(\mathfrak{o}_{N}\right)$ através da seguinte expansão:

$$
\gamma_{m} N \operatorname{tr}_{1, \ldots, m} H^{(m)}(\tau+F[-1])_{1} \cdots(\tau+F[-1])_{m}=\phi_{m 0} \tau^{m}+\phi_{m 1} \tau^{m-1}+\cdots+\phi_{m m}
$$

onde $\tau+F[-1]=\left(\delta_{i j} \tau+F_{i j}[-1]\right)$.

Proposição 3.5.2. $\tau+F[-1]$ é uma matriz de Manin.

Demonstração. Ver lema 8.1.5. da página 123 de Molev [2018].

Teorema 3.5.1. Todos elementos $\phi_{m a}$ pertencem ao centro de Feigin-Frenkel $\mathfrak{z}\left(\widehat{o}_{n}\right)$.

Demonstração. Ver Molev [2013].

Teorema 3.5.2. $\left\{\phi_{22}, \phi_{44}, \ldots, \phi_{2 n 2 n}\right\}$ é um conjunto completo de vetores de Segal-Sugawara para $\mathfrak{o}_{2 n+1}$.

$\left\{\phi_{22}, \phi_{44}, \ldots, \phi_{2 n-22 n-2}, \operatorname{PfF}[-1]\right\}$ é um conjunto completo de vetores de Segal-Sugawara para $\mathfrak{o}_{2 n}$.

Demonstração. Ver Molev [2013].

\subsection{Tipo C}

Nessa seção vamos focar o estudo para as álgebras de Lie de tipo $C$.

Seja $\mathfrak{g}=\mathfrak{s p}_{N}=\mathfrak{s p}_{2 n}$ a álgebra de Lie simplética (tipo $C$ ). $\mathfrak{s p}_{N}$ pode ser vista como uma subálgebra de $\mathfrak{g l}_{N}$, gerada pelos elementos $F_{i j}:=E_{i j}-\epsilon_{i} \epsilon_{j} E_{j^{\prime} i^{\prime}}$, onde $i^{\prime}=N-i+1$ e:

$$
\epsilon_{i}:=\left\{\begin{array}{l}
1, \text { se } i \in\{1, \ldots, n\} \\
-1, \text { se } i \in\{n+1, \ldots, N\}
\end{array}\right.
$$

para todo $i, j \in\{1, \ldots, N\}$.

\subsubsection{Geradores do centro de Feigin-Frenkel}

O número de Coxeter dual para a álgebra de Lie ortogonal é $h^{\vee}=n+1$. Assim, a forma de Killing normalizada é dada por

$$
\langle X, Y\rangle=\operatorname{tr} X Y
$$

para todos $X, Y \in \mathfrak{s p}_{2 n}$.

Considere a álgebra de Kac-Moody afim $\widehat{\mathfrak{s p}_{2 n}}=\mathfrak{s p}_{2 n}\left[t, t^{-1}\right] \oplus \mathbb{C} K$. Denote por $F_{i j}[r]$ os elementos $F_{i j} t^{r}$, para todo $r \in \mathbb{Z}$. O colchete de $\widehat{\mathfrak{s p}_{2 n}}$ é definido como:

$$
\begin{aligned}
{\left[F_{i j}[r], F_{k l}[s]\right]=} & \delta_{k j} F_{i l}[r+s]-\delta_{i l} F_{k j}[r+s]-\epsilon_{i} \epsilon_{j}\left(\delta_{k i^{\prime}} F_{j^{\prime} l}[r+s]+\delta_{j^{\prime} l} F_{k i^{\prime}}[r+s]\right)+ \\
& +2 r \delta_{r,-s} K\left(\delta_{k j} \delta_{i l}-\epsilon_{i} \epsilon_{j} \delta_{k i^{\prime}} \delta_{j^{\prime} l}\right)
\end{aligned}
$$

onde $K$ é um elemento central.

Para todo $r \in \mathbb{Z}$ defina o seguinte elemento:

$$
F[r]=\sum_{i, j=1}^{2 n} e_{i j} \otimes F_{i j}[r]
$$

pertencente a $\operatorname{End} \mathbb{C}^{2 n} \otimes \mathrm{U}\left(\widehat{\mathfrak{s p}_{2 n}}\right)$. 
Para $a, m \in\{1, \ldots, 2 n\}$, com $a \leq m$, defina o elemento:

$$
F[r]_{a}=\sum_{i, j=1}^{2 n} 1^{\otimes(a-1)} \otimes e_{i j} \otimes 1^{\otimes(m-a)} \otimes F_{i j}[r]
$$

pertencente a $\left(\operatorname{End} \mathbb{C}^{2 n}\right)^{\otimes m} \otimes \mathrm{U}\left(\widehat{\mathfrak{s p}_{2 n}}\right)$.

Iremos trabalhar com a álgebra de Lie estendida $\widehat{\mathfrak{s p}_{2 n}} \oplus \mathbb{C} \tau$ onde o elemento $\tau$ satisfaz as seguintes relações:

$$
[\tau, X[r]]=-r X[r-1] \quad, \quad[\tau, K]=0 .
$$

Defina a seguinte constante

$$
\gamma_{m}(-2 n)=\frac{2 n-m+2}{2(n-m+1)}
$$

Considere o módulo vácuo no nível crítico $V_{-h^{\vee}}\left(\mathfrak{s p}_{2 n}\right) \cong \mathrm{U}\left(t^{-1} \mathfrak{s p}_{2 n}\left[t^{-1}\right]\right)$. Defina os elementos $\phi_{m a}$ de $V_{-h^{\vee}}\left(\mathfrak{s p}_{2 n}\right)$ através da seguinte expansão:

$$
\gamma_{m}(-2 n) \operatorname{tr}_{1, \ldots, m} H^{(m)}(\tau+F[-1])_{1} \cdots(\tau+F[-1])_{m}=\phi_{m 0} \tau^{m}+\phi_{m 1} \tau^{m-1}+\cdots+\phi_{m m}
$$

onde $\tau+F[-1]=\left(\delta_{i j} \tau+F_{i j}[-1]\right)$.

Proposição 3.6.1. $\tau+F[-1]$ é uma matriz de Manin.

Demonstração. Ver lema 8.3.1. da página 135 de Molev [2018].

Teorema 3.6.1. Seja $n \geq m$. Então todos elementos $\phi_{m a}$ pertencem ao centro de Feigin-Frenkel $\mathfrak{z}\left(\widehat{s p}_{2 n}\right)$.

Demonstração. Ver Molev [2013].

Teorema 3.6.2. $\left\{\phi_{22}, \phi_{44}, \ldots, \phi_{2 n 2 n}\right\}$ é um conjunto completo de vetores de Segal-Sugawara para $\mathfrak{s p}_{2 n}$.

Demonstração. Ver Molev [2013].

\section{7 $\mathrm{U}(\mathfrak{g})$ é livre sobre $A_{\mu}$ ?}

Definição 3.7.1. Uma álgebra $U$ é chamada de álgebra $P B W$ se qualquer elemento de $U$ pode ser escrito de forma única como uma combinação linear de monômios ordenados em alguns geradores fixados de $U$.

Definição 3.7.2. Uma $P B W$ álgebra $U$ é chamada de filtrada especial se sua álgebra graduada $g r(U)$ é uma álgebra de polinômios.

Exemplo 3.7.1. Pelo teorema de Poincaré-Birkhoff-Witt temos que toda álgebra envolvente universal de uma álgebra de Lie de dimensão finita é uma álgebra filtrada especial.

Teorema 3.7.1. Sejam $U$ uma álgebra filtrada especial e $g_{1}, \ldots, g_{t} \in U$ elementos mutuamente comutativos. Denote por $\overline{g_{i}}$ a imagem de $g_{i}$ em $g r(U), i \in\{1, \ldots, t\}$. Se $\overline{g_{1}}, \ldots, \overline{g_{t}}$ é uma intersecção completa para gr $(U)$, então $U$ é livre como $k\left[g_{1}, \ldots, g_{t}\right]$-módulo à esquerda (ou à direita).

Demonstração. Ver Futorny e Ovsienko [2003].

Uma consequência desse teorema é que $\mathrm{U}(\mathfrak{g})$ é livre sobre seu centro.

Se os geradores $P_{i, \mu}^{(j)}$ (como em (3.5)) de $\overline{A_{\mu}}$ formarem uma sequência regular, pelo teorema acima e pela proposição 2.7.2 temos que, se existir $A_{\mu}$ tal que $\operatorname{gr}\left(A_{\mu}\right)=\overline{A_{\mu}}$, então $\mathrm{U}(\mathfrak{g})$ é um $A_{\mu}$-módulo livre. Para $\mathfrak{g}=\mathfrak{s l}_{n}$ e $\mu$ regular semissimples, isso é discutido na observação 3.4 de Panyushev e Yakimova [2008]. 
Teorema 3.7.2. Seja $\mathfrak{g}$ uma álgebra de Lie redutivel e $\mu$ regular, então os geradores de $\overline{A_{\mu}}$ formam uma sequência regular.

Demonstração. Ver teorema 1.2 de Moreau [2018]. 


\section{Capítulo 4}

\section{Subálgebras de Gelfand-Tsetlin}

\subsection{Definição}

Definição 4.1.1. Seja $\mathfrak{g l}_{n}$ a álgebra de Lie linear geral (tipo A), que consiste das matrizes $n \times n$, com base canônica $\left\{E_{i j} \mid 1 \leq i, j \leq n\right\}$. Para $1 \leq m \leq n$, considere $\mathfrak{g l}_{m}$ a álgebra de Lie gerada por $\left\{E_{i j} \mid 1 \leq i, j \leq m\right\}$. Assim temos:

$$
\mathfrak{g l}_{1} \subset \mathfrak{g l}_{2} \subset \ldots \subset \mathfrak{g l}_{n}
$$

$e$ :

$$
U\left(\mathfrak{g l}_{1}\right) \subset U\left(\mathfrak{g l}_{2}\right) \subset \ldots \subset U\left(\mathfrak{g l}_{n}\right) .
$$

Denote o centro de $U\left(\mathfrak{g l}_{m}\right)$ por $Z_{m}$ para todo $m \in\{1, \ldots, n\}$. A subálgebra de Gelfand-Tsetlin $\Gamma$ de $U\left(\mathfrak{g l}_{n}\right)$ é definida como a subálgebra gerada por $\left\{Z_{1}, \ldots, Z_{n}\right\}$.

Teorema 4.1.1. Para $m \in\{1, \ldots, n\}$, o centro $Z_{m}$ é uma álgebra polinomial em $m$ variáveis $\left\{\lambda_{m j} \mid j=1,2, \ldots, m\right\}$ com

$$
\lambda_{m j}=\sum_{t_{1}, \ldots, t_{m} \in 1, \ldots, i} E_{t_{1} t_{2}} E_{t_{2} t_{3}} \cdots E_{t_{j-1} t_{j}} E_{t_{j}, t_{1}}
$$

Além disso, a subálgebra $\Gamma$ é uma álgebra de polinômios nas $\frac{n(n+1)}{2}$ variáveis $\left\{\lambda_{i j} \mid 1 \leq j \leq i \leq n\right\}$. Demonstração. Ver página 169 de Zelobenko [1973].

Seja $\overline{\lambda_{i j}}$ a imagem de $\lambda_{i j} \operatorname{em~} \operatorname{gr}\left(\mathrm{U}\left(\mathfrak{g l}_{n}\right)\right)$.

Definição 4.1.2. A variedade de Gelfand-Tsetlin para $\mathfrak{g l}_{n}$ é a variedade algébrica:

$$
\mathfrak{G}_{\mathfrak{g l}_{n}}:=\mathcal{V}\left(\left\{\overline{\lambda_{i j}} \mid i=1, \ldots, n, j=1, \ldots, i\right\}\right) \subset k^{n^{2}}
$$

Teorema 4.1.2. A variedade de Gelfand-Tsetlin para $\mathfrak{g l}_{n}$ é equidimensional de dimensão $\frac{n(n-1)}{2}$. Demonstração. Ver demonstração em Ovsienko [2003].

\subsection{Relação com subálgebras de Mishchenko-Fomenko}

Sejam $\lambda, \mu \in \mathfrak{g}^{*}$, com $\mu$ regular e $\lambda$ não, e $u \in \mathbb{C}$. Em Vinberg [2014] e Molev e Yakimova [2017] são discutidos o limite de $\bar{A}_{\lambda+u \mu}$ para $u$ tendendo a 0 . Para $\mathfrak{g}$ do tipo $A$, o limite é a álgebra graduada da álgebra de Gelfand-Tsetlin. Para o tipo $C$, podemos definir uma álgebra cuja graduação é o limite de $\bar{A}_{\lambda+u \mu}$ para $u$ tendendo a 0 e a chamaremos de álgebra de Gelfand-Tsetlin (para o tipo $C$ ). Os geradores dessa álgebra podem ser encontrados em Molev e Yakimova [2017] e trabalharemos com eles na próxima seção. 


\subsection{Subálgebras de Gelfand-Tsetlin para o tipo $C$}

Seja $\mathfrak{g}=\mathfrak{s p}_{N}=\mathfrak{s p}_{2 n}$ a álgebra linear simplética. $\mathfrak{s p}_{n}$ pode ser vista como uma subálgebra de $\mathfrak{g l}_{N}$, gerada pelos elementos $F_{i j}:=E_{i j}-\epsilon_{i} \epsilon_{j} E_{j^{\prime} i^{\prime}}$, onde $i^{\prime}=N-i+1$ e:

$$
\epsilon_{i}:=\left\{\begin{array}{l}
1, \text { se } i \in\{1, \ldots, n\} \\
-1, \text { se } i \in\{n+1, \ldots, N\}
\end{array}\right.
$$

para todo $i, j \in\{1, \ldots, N\}$.

Proposição 4.3.1. Considere $F=\left(F_{i j}\right)$, mas, abusando da notação, aqui $F_{i j}$ representa a imagem de $F_{i j}$ em $S\left(\mathfrak{s p}_{2 \mathfrak{n}}\right)$. Considere também o polinômio característico:

$$
\operatorname{det}(u+F)=u^{2 n}+\phi_{2} u^{2 n-2}+\cdots+\phi_{2 n} .
$$

Os coeficientes $\phi_{2}, \ldots, \phi_{2 n}$ são geradores algebricamente independentes da álgebra de $\mathfrak{s p}_{2 n}$-invariantes $S\left(\mathfrak{s p}_{2 n}\right)^{\mathfrak{s p}_{2 n}}$.

Demonstração. Página 29 e 30 de Molev [2018].

Exemplo 4.3.1. Para $\mathfrak{s p}_{2}$ temos:

$$
\begin{gathered}
F_{11}=E_{11}-E_{22}, \quad F_{12}=2 E_{12}, \quad F_{21}=2 E_{21}, \quad F_{22}=E_{22}-E 11=-F_{11} \\
\operatorname{det}(u+F)=\operatorname{det}\left(u I d+\left(\begin{array}{cc}
F_{11} & F_{12} \\
F_{21} & -F_{11}
\end{array}\right)\right)=\left(u+F_{11}\right)\left(u-F_{11}\right)-F_{12} F_{21}=u^{2}+\left(-F_{11}^{2}-F_{12} F_{21}\right)
\end{gathered}
$$

Portanto, $S\left(\mathfrak{s p}_{2}\right)^{\mathfrak{s p}_{2}}$ é gerada por $\phi_{2}=-F_{11}^{2}-F_{12} F_{21}$.

Exemplo 4.3.2. Para $\mathfrak{s p}_{4}$ temos:

$$
\begin{array}{ll}
F_{11}=E_{11}-E_{44}=-F_{44}, & F_{12}=E_{12}-E_{34}=-F_{34}, \quad F_{13}=E_{13}+E_{24}=F_{24}, \\
F_{14}=E_{14}+E_{14}=2 E_{14}, & F_{21}=E_{21}-E 43=-F_{43}, \quad F_{22}=E_{22}-E_{33}=-F_{33}, \\
F_{23}=E_{23}+E_{23}=2 E_{23}, & F_{31}=E_{31}+E_{42}=F_{42}, \quad F_{32}=E_{32}+E_{32}=2 E_{32}, \\
& F_{41}=E_{41}+E_{41}=2 E_{41}
\end{array}
$$




$$
\begin{aligned}
& \operatorname{det}(u+F)=\operatorname{det}\left(\begin{array}{cccc}
u+F_{11} & F_{12} & F_{13} & F_{14} \\
F_{21} & u+F_{22} & F_{23} & F_{13} \\
F_{31} & F_{32} & u-F_{22} & -F_{12} \\
F_{41} & F_{31} & -F_{21} & u-F_{11}
\end{array}\right) \\
& =\left(u+F_{11}\right) \operatorname{det}\left(\begin{array}{ccc}
u+F_{22} & F_{23} & F_{13} \\
F_{32} & u-F_{22} & -F_{12} \\
F_{31} & -F_{21} & u-F_{11}
\end{array}\right)-F_{12} \operatorname{det}\left(\begin{array}{ccc}
F_{21} & F_{23} & F_{13} \\
F_{31} & u-F_{22} & -F_{12} \\
F_{41} & -F_{21} & u-F_{11}
\end{array}\right) \\
& +F_{13} \operatorname{det}\left(\begin{array}{ccc}
F_{21} & u+F_{22} & F_{13} \\
F_{31} & F_{32} & -F_{12} \\
F_{41} & F_{31} & u-F_{11}
\end{array}\right)-F_{14} \operatorname{det}\left(\begin{array}{ccc}
F_{21} & u+F_{22} & F_{23} \\
F_{31} & F_{32} & u-F_{22} \\
F_{41} & F_{31} & -F_{21}
\end{array}\right) \\
& =\left(u+F_{11}\right)\left(u^{3}-F_{11} u^{2}-F_{22}^{2} u-F_{13} F_{31} u-F_{23} F_{32} u-F_{12} F_{21} u+\right. \\
& \left.+F_{22}^{2} F_{11}-F_{23} F_{12} F_{31}-F_{13} F_{32} F_{21}+F_{13} F_{22} F_{31}+F_{23} F_{32} F_{11}-F_{22} F_{12} F_{21}\right) \\
& -F_{12}\left(F_{21} u^{2}-F_{21} F_{22} u-F_{21} F_{11} u-F_{13} F_{41} u-F_{23} F_{31} u+\right. \\
& \left.+F_{21} F_{22} F_{11}-F_{23} F_{12} F_{41}-F_{13} F_{31} F_{21}+F_{13} F_{22} F_{41}+F_{23} F_{31} F_{11}-F_{21} F_{12} F_{21}\right) \\
& +F_{13}\left(-F_{31} u^{2}+F_{21} F_{32} u-F_{12} F_{41} u+F_{31} F_{11} u-F_{22} F_{31} u-\right. \\
& \left.-F_{21} F_{32} F_{11}-F_{22} F_{12} F_{41}+F_{13} F_{31}^{2}-F_{13} F_{32} F_{41}+F_{22} F_{31} F_{11}+F_{21} F_{12} F_{31}\right) \\
& -F_{14}\left(F_{41} u^{2}+F_{31} F_{21} u-F_{21} F_{31} u-\right. \\
& \left.-F_{21} F_{32} F_{21}-F_{22}^{2} F_{41}+F_{23} F_{31}^{2}-F_{23} F_{32} F_{41}+F_{22} F_{31} F_{21}+F_{21} F_{22} F_{31}\right) \\
& \stackrel{*}{=} u^{4}+\left(-F_{11}^{2}-F_{22}^{2}-2 F_{12} F_{21}-2 F_{13} F_{31}-F_{23} F_{32}-F_{14} F_{41}\right) u^{2} \\
& +F_{11} F_{22}^{2} F_{11}-F_{11} F_{23} F_{12} F_{31}-F_{11} F_{13} F_{32} F_{21}+F_{11} F_{13} F_{22} F_{31}+F_{11} F_{23} F_{32} F_{11}- \\
& -F_{11} F_{22} F_{12} F_{21}-F_{12} F_{21} F_{22} F_{11}+F_{12} F_{23} F_{12} F_{41}+F_{12} F_{13} F_{31} F_{21}-F_{12} F_{13} F_{22} F_{41}- \\
& -F_{12} F_{23} F_{31} F_{11}+F_{12} F_{21} F_{12} F_{21}-F_{13} F_{21} F_{32} F_{11}-F_{13} F_{22} F_{12} F_{41}+F_{13}^{2} F_{31}^{2}- \\
& -F_{13}^{2} F_{32} F_{41}+F_{13} F_{22} F_{31} F_{11}+F_{13} F_{21} F_{12} F_{31}+F_{14} F_{21} F_{32} F_{21}+F_{14} F_{22}^{2} F_{41}- \\
& -F_{14} F_{23} F_{31}^{2}+F_{14} F_{23} F_{32} F_{41}-F_{14} F_{22} F_{31} F_{21}-F_{14} F_{21} F_{22} F_{31} \\
& \stackrel{*}{=} u^{4}+\left(-F_{11}^{2}-F_{22}^{2}-2 F_{12} F_{21}-2 F_{13} F_{31}-F_{23} F_{32}-F_{14} F_{41}\right) u^{2}+ \\
& +F_{11}^{2} F_{22}^{2}+F_{12}^{2} F_{21}^{2}+F_{13}^{2} F_{31}^{2}+F_{11}^{2} F_{23} F_{32}+F_{12}^{2} F_{23} F_{41}-F_{13}^{2} F_{32} F_{41}+F_{14} F_{21}^{2} F_{32}+ \\
& +F_{14} F_{22}^{2} F_{41}-F_{14} F_{23} F_{31}^{2}-2 F_{11} F_{12} F_{21} F_{22}-2 F_{11} F_{12} F_{23} F_{31}-2 F_{11} F_{13} F_{21} F_{32}+ \\
& +2 F_{11} F_{13} F_{22} F_{31}+2 F_{12} F_{13} F_{21} F_{31}-2 F_{12} F_{13} F_{22} F_{41}-2 F_{14} F_{21} F_{22} F_{31}+F_{14} F_{23} F_{32} F_{41}
\end{aligned}
$$

(* Lembre-se que estamos fazendo essas contas em $S\left(\mathfrak{s p}_{4}\right)$, então os $F_{i j}$ 's comutam entre si.)

Portanto, $S\left(\mathfrak{s p}_{4}\right)^{\mathfrak{s p}_{4}}$ é gerada por:

$$
\begin{aligned}
\phi_{2} & =-F_{11}^{2}-F_{22}^{2}-2 F_{12} F_{21}-2 F_{13} F_{31}-F_{23} F_{32}-F_{14} F_{41} \\
\phi_{4} & =F_{11}^{2} F_{22}^{2}+F_{12}^{2} F_{21}^{2}+F_{13}^{2} F_{31}^{2}+F_{11}^{2} F_{23} F_{32}+F_{12}^{2} F_{23} F_{41}-F_{13}^{2} F_{32} F_{41}+F_{14} F_{21}^{2} F_{32}+ \\
& +F_{14} F_{22}^{2} F_{41}-F_{14} F_{23} F_{31}^{2}-2 F_{11} F_{12} F_{21} F_{22}-2 F_{11} F_{12} F_{23} F_{31}-2 F_{11} F_{13} F_{21} F_{32}+ \\
& +2 F_{11} F_{13} F_{22} F_{31}+2 F_{12} F_{13} F_{21} F_{31}-2 F_{12} F_{13} F_{22} F_{41}-2 F_{14} F_{21} F_{22} F_{31}+F_{14} F_{23} F_{32} F_{41}
\end{aligned}
$$


Exemplo 4.3.3. Para $\mathfrak{s p}_{6}$ temos:

$$
\begin{aligned}
& F_{11}=E_{11}-E_{66}=-F_{66}, \quad F_{12}=E_{12}-E_{56}=-F_{56}, \quad F_{13}=E_{13}-E_{46}=-F_{46}, \\
& F_{14}=E_{14}+E_{36}=F_{36}, \quad F_{15}=E_{15}+E_{26}=F_{26}, \quad F_{16}=2 E_{16}, \\
& F_{21}=E_{21}-E_{65}=-F_{65}, \quad F_{22}=E_{22}-E_{55}=-F_{55}, \quad F_{23}=E_{23}-E_{45}=-F_{45}, \\
& F_{24}=E_{24}+E_{35}=F_{35}, \quad F_{25}=2 E_{25}, \quad F_{31}=E_{31}-E_{64}=-F_{64}, \\
& F_{32}=E_{32}-E_{54}=-F_{54}, \quad F_{33}=E_{33}-E_{44}=-F_{44}, \quad F_{34}=2 E_{34}, \\
& F_{41}=E_{41}+E_{63}=F_{63}, F_{42}=E_{42}+E_{53}=F_{53}, \quad F_{43}=2 E_{43}, \\
& F_{51}=E_{51}+E_{62}=F_{62}, \quad F_{52}=2 E_{52}, \quad F_{61}=2 E_{61} \text {. } \\
& \operatorname{det}(u+F)=\operatorname{det}\left(\begin{array}{cccccc}
u+F_{11} & F_{12} & F_{13} & F_{14} & F_{15} & F_{16} \\
F_{21} & u+F_{22} & F_{23} & F_{24} & F_{25} & F_{15} \\
F_{31} & F_{32} & u+F_{33} & F_{34} & F_{24} & F_{14} \\
F_{41} & F_{42} & F_{43} & u-F_{33} & -F_{23} & -F_{13} \\
F_{51} & F_{52} & F_{42} & -F_{32} & u-F_{22} & -F_{12} \\
F_{61} & F_{51} & F_{41} & -F_{31} & -F_{21} & u-F_{11}
\end{array}\right)
\end{aligned}
$$

Foi usado um programa para calcular o determinante dessa matriz que gerou um documento de texto de quatro páginas (veja mais detalhes no apêndice 1), então o resultado não será mostrado aqui.

Definição 4.3.1. Considere a função $\varpi: S(\mathfrak{g}) \rightarrow U(\mathfrak{g})$, tal que

$$
x_{1} \cdots x_{k} \mapsto \frac{1}{k !} \sum_{\sigma \in S_{k}} \widehat{x_{\sigma(1)}} \cdots \widehat{x_{\sigma(k)}}
$$

onde $x_{1} \cdots x_{k}$ é o produto das imagens de $x_{1}, \ldots, x_{n} \in \mathfrak{g}$ em $S(\mathfrak{g})$ e $\widehat{x_{i}}$ é a imagem de $x_{i}$ em $U(\mathfrak{g})$. Chamamos $\varpi$ de função simetrizante.

Proposição 4.3.2. $\varpi$ é um isomorfismo de espaços vetoriais filtrados.

Demonstração. Ver teorema 5.4 da página 114 de Meinrenken [2013].

Definição 4.3.2. Seja $H \in S(\mathfrak{g})=\mathbb{C}\left[\mathfrak{g}^{*}\right]$ e $\lambda \in \mathfrak{g}^{*}$, a derivada direcional de $H$ com respeito a $\lambda$, $\partial_{\lambda} H$, é definida da seguinte maneira:

$$
\partial_{\lambda} H(x)=\left.\frac{d}{d t} H(x+t \lambda)\right|_{t=0} .
$$

Proposição 4.3.3. O centro de $U\left(\mathfrak{s p}_{2 n}\right)$, denotado por $Z\left(U\left(\mathfrak{s p}_{2 n}\right)\right)$, é gerado por $\left\{\varpi\left(\phi_{2}\right), \ldots, \varpi\left(\phi_{2 n}\right)\right\}$.

Demonstração. Vide página 26 de Molev e Yakimova [2017].

Para $\mathfrak{s p}_{2 n}$ :

(i) Para $m \in\{0, \ldots, n-1\}$, podemos ver $\mathfrak{s p}_{2 n-2 m}$ como o subálgebra de $\mathfrak{s p}_{2 n}$ gerado pelos elementos $F_{i j}$ para todos $i, j \in\left\{m+1, \ldots,(m+1)^{\prime}\right\}$ (lembrando que $\left.(m+1)^{\prime}=n-m\right)$.

(ii) Considere $h(i-1)=F_{i i}$, para todo $i \in\{1, \ldots, n\}$, e denote por $\phi_{2 i}^{(m)} \in S\left(\mathfrak{s p}_{2 n-2 m}\right)$ o $2 i$-ésimo coeficiente do polinômio característico associado a $\mathfrak{s p}_{2 n-2 m}$, para todos $m \in\{0, \ldots, n-1\}$ e $i \in\{1, \ldots, n-m\}$. 
Definição 4.3.3. A subálgebra de Gelfand-Tsetlin para $\mathfrak{s p}_{2 n}$ é definida como a subálgebra de $U\left(\mathfrak{s p}_{2 n}\right)$ livremente gerada pelos centros de $U\left(\mathfrak{s p}_{2 k}\right)$, para $1 \leq k \leq n$, e pelos elementos $\varpi\left(\partial_{h(m)} \phi_{2 i}^{(m)}\right)$, para $m \in\{0, \ldots, n-1\}$ e $i \in\{1, \ldots, n-m\}$. Ou seja, é a subálgebra gerada por:

$$
\left\{\varpi\left(\phi_{2 i}^{(m)}\right) \mid 0 \leq m \leq n-1,1 \leq i \leq n-m\right\} \cup\left\{\varpi\left(\partial_{h(m)} \phi_{2 i}^{(m)}\right) \mid 0 \leq m \leq n-1,1 \leq i \leq n-m\right\} .
$$

Denote por $\bar{x}$ a imagem de $x \in U\left(\mathfrak{s p}_{2 n}\right)$ em $\operatorname{gr}\left(U\left(\mathfrak{s p}_{2 n}\right)\right)=S\left(\mathfrak{s p}_{2 n}\right)$. Note que, pela definição da função simetrizante, temos $\overline{\varpi(y)}=y$ para todo $y \in S\left(\mathfrak{s p}_{2 n}\right)$. Dessa forma, temos a seguinte definição:

Definição 4.3.4. A variedade de Gelfand-Tsetlin para $\mathfrak{s p}_{2 n}$, denotada por $\mathfrak{G}_{\mathfrak{s p}_{2 n}}$, é a variedade algébrica dada por:

$\mathcal{V}\left(\left\{\phi_{2 i}^{(m)} \mid 0 \leq m \leq n-1,1 \leq i \leq n-m\right\} \cup\left\{\partial_{h(m)} \phi_{2 i}^{(m)} \mid 0 \leq m \leq n-1,1 \leq i \leq n-m\right\}\right) \subset k^{n(2 n+1)}$

sendo $n(2 n+1)$ a dimensão de $\mathfrak{s p}_{2 n}$ como espaço vetorial.

Exemplo 4.3.4. Vamos calcular $\mathfrak{G}_{\mathfrak{s p}_{4}}$.

- $m=1$ (coeficientes em $\mathfrak{s p}_{2}$ ):

No exemplo 4.0.1 já calculamos que:

$$
\phi_{2}^{(1)}=-F_{11}^{2}-F_{12} F_{21} .
$$

Mas, como $m=1$ e $\mathfrak{s p}_{2}$ é vista como a subálgebra de $\mathfrak{s p}_{4}$ gerada por $F_{i, j}$ com $i, j \in\{2,3\}$, devemos trocar 1 por 2 e 2 por 3. Assim temos:

$$
\begin{aligned}
& \phi_{2}^{(1)}=-F_{22}^{2}-F_{23} F_{32} \\
& \partial_{h(1)} \phi_{2}^{(1)}=\partial_{F_{22}}\left(-F_{22}^{2}-F_{23} F_{32}\right)=-2 F_{22}
\end{aligned}
$$

- $m=0$ (coeficientes em $\mathfrak{s p}_{4}$ ):

No exemplo 4.0.2 já calculamos que:

$$
\begin{aligned}
\phi_{2}^{(0)} & =-F_{11}^{2}-F_{22}^{2}-2 F_{12} F_{21}-2 F_{13} F_{31}-F_{23} F_{32}-F_{14} F_{41} \\
\phi_{4}^{(0)} & =F_{11}^{2} F_{22}^{2}+F_{12}^{2} F_{21}^{2}+F_{13}^{2} F_{31}^{2}+F_{11}^{2} F_{23} F_{32}+F_{12}^{2} F_{23} F_{41}-F_{13}^{2} F_{32} F_{41}+F_{14} F_{21}^{2} F_{32}+ \\
& +F_{14} F_{22}^{2} F_{41}-F_{14} F_{23} F_{31}^{2}-2 F_{11} F_{12} F_{21} F_{22}-2 F_{11} F_{12} F_{23} F_{31}-2 F_{11} F_{13} F_{21} F_{32}+ \\
& +2 F_{11} F_{13} F_{22} F_{31}+2 F_{12} F_{13} F_{21} F_{31}-2 F_{12} F_{13} F_{22} F_{41}-2 F_{14} F_{21} F_{22} F_{31}+F_{14} F_{23} F_{32} F_{41}
\end{aligned}
$$

Temos:

$$
\partial_{h(0)} \phi_{2}^{(0)}=\partial_{F_{11}}\left(-F_{11}^{2}-F_{22}^{2}-2 F_{12} F_{21}-2 F_{13} F_{31}-F_{23} F_{32}-F_{14} F_{41}\right)=-2 F_{11}
$$

$e$ :

$$
\begin{aligned}
\partial_{h(0)} \phi_{4}^{(0)} & =\partial_{F_{11}}\left(F_{11}^{2} F_{22}^{2}+F_{12}^{2} F_{21}^{2}+F_{13}^{2} F_{31}^{2}+F_{11}^{2} F_{23} F_{32}+F_{12}^{2} F_{23} F_{41}-F_{13}^{2} F_{32} F_{41}+F_{14} F_{21}^{2} F_{32}+\right. \\
& +F_{14} F_{22}^{2} F_{41}-F_{14} F_{23} F_{31}^{2}-2 F_{11} F_{12} F_{21} F_{22}-2 F_{11} F_{12} F_{23} F_{31}-2 F_{11} F_{13} F_{21} F_{32}+ \\
& \left.+2 F_{11} F_{13} F_{22} F_{31}+2 F_{12} F_{13} F_{21} F_{31}-2 F_{12} F_{13} F_{22} F_{41}-2 F_{14} F_{21} F_{22} F_{31}+F_{14} F_{23} F_{32} F_{41}\right) \\
& =2 F_{11} F_{22}^{2}+2 F_{11} F_{23} F_{32}-2 F_{12} F_{21} F_{22}-2 F_{12} F_{23} F_{31}-2 F_{13} F_{21} F_{32}+2 F_{13} F_{22} F_{31}
\end{aligned}
$$

Outro jeito de calcular $\partial_{h(0)} \phi_{4}^{(0)}$ é da seguinte forma:

Seja $M=\left(m_{i j}\right)$ uma matriz 4 por 4. Sabemos que $\operatorname{det} M=\sum_{\sigma \in S_{4}} \operatorname{sgn}(\sigma) m_{1 \sigma(1)} m_{2 \sigma(2)} m_{3 \sigma(3)} m_{4 \sigma(4)}$.

Como $\phi_{4}^{(0)}$ é o coeficiente do determinante que não acompanha $u$, temos $\phi_{4}^{(0)}=\operatorname{det}(F)=$ 
$\sum_{\sigma \in S_{4}} \operatorname{sgn}(\sigma) F_{1 \sigma(1)} F_{2 \sigma(2)} F_{3 \sigma(3)} F_{4 \sigma_{4}}$. Para calcular $\partial_{h(0)} \phi_{4}^{(0)}$ não importa os monômios de $\phi_{4}^{(0)}$ que não têm $F_{11}$, pois esses serão zerados no cáldulo da derivada direcional. Além disso, em $\mathfrak{s p}_{4}$ temos $F_{11}=-F_{44}$. Então podemos nos concentrar nos monômios formados por

$\sum_{\substack{\sigma \in S_{4} \\ \sigma(1)=1}} \operatorname{sgn}(\sigma) F_{1 \sigma(1)} F_{2 \sigma(2)} F_{3 \sigma(3)} F_{4 \sigma_{4}} e \sum_{\substack{\sigma \in S_{4} \\ \sigma(4)=4}} \operatorname{sgn}(\sigma) F_{1 \sigma(1)} F_{2 \sigma(2)} F_{3 \sigma(3)} F_{4 \sigma_{4}}$, ou seja:

$$
F_{11} \operatorname{det}\left(\begin{array}{ccc}
F_{22} & F_{23} & F_{13} \\
F_{32} & -F_{22} & -F_{12} \\
F_{31} & -F_{21} & -F_{11}
\end{array}\right) e-F_{11} \operatorname{det}\left(\begin{array}{ccc}
F_{11} & F_{12} & F_{13} \\
F_{21} & F_{22} & F_{23} \\
F_{31} & F_{32} & -F_{22}
\end{array}\right) \text {. }
$$

Os monômios formados dessa forma terão ou $F_{11}$ ou $F_{11}^{2}$. Se tiver $F_{11}$, ele irá aparecer em somente uma das fórmulas acima e sua derivada direcional em $F_{11}$ é igual dividí-lo por $F_{11}$. Se tiver $F_{1}^{2}$, irá aparecer uma vez em cada uma das fórmulas acima e sua derivada direcional em $F_{11}$ é igual a dividí-lo por $F_{11}$ e multiplicar o resultado por 2. Assim concluimos que:

$$
\begin{aligned}
& \partial_{h(0)} \phi_{4}^{(0)}=\operatorname{det}\left(\begin{array}{ccc}
F_{22} & F_{23} & F_{13} \\
F_{32} & -F_{22} & -F_{12} \\
F_{31} & -F_{21} & -F_{11}
\end{array}\right)-\operatorname{det}\left(\begin{array}{ccc}
F_{11} & F_{12} & F_{13} \\
F_{21} & F_{22} & F_{23} \\
F_{31} & F_{32} & -F_{22}
\end{array}\right)= \\
& =2 F_{11} F_{22}^{2}+2 F_{11} F_{23} F_{32}-2 F_{12} F_{21} F_{22}-2 F_{12} F_{23} F_{31}-2 F_{13} F_{21} F_{32}+2 F_{13} F_{22} F_{31}
\end{aligned}
$$

Portanto, são geradores de $\mathfrak{G}_{\mathfrak{s p}_{4}}$ :

$$
\begin{aligned}
& F_{11} \\
& F_{22} \\
& F_{23} F_{32} \\
& 2 F_{12} F_{21}+2 F_{13} F_{31}+F_{14} F_{41} \\
& F_{12} F_{23} F_{31}+F_{13} F_{21} F_{32} \\
& F_{12}^{2} F_{21}^{2}+F_{13}^{2} F_{31}^{2}+F_{12}^{2} F_{23} F_{41}-F_{13}^{2} F_{32} F_{41}+F_{14} F_{21}^{2} F_{32}-F_{14} F_{23} F_{31}^{2}+2 F_{12} F_{13} F_{21} F_{31}
\end{aligned}
$$

pela definição de variedade e pelas seguintes igualdades:

$$
\begin{aligned}
& F_{11}=-\frac{1}{2} \partial_{h(0)} \phi_{2}^{(0)} \\
& F_{22}=-\frac{1}{2} \partial_{h(1)} \phi_{2}^{(1)} \\
& F_{23} F_{32}=-\phi_{2}^{(1)}-F_{22}^{2} \\
& 2 F_{12} F_{21}+2 F_{13} F_{31}+F_{14} F_{41}=-\phi_{2}^{(0)}-F_{11}^{2}-F_{22}^{2}-F_{23} F_{32} \\
& F_{12} F_{23} F_{31}+F_{13} F_{21} F_{32}=-\frac{1}{2} \partial_{h(0)} \phi_{4}^{(0)}+F_{11} F_{22}^{2}+F_{11}\left(F_{23} F_{32}\right)-\left(F_{12} F_{21}\right) F_{22}+\left(F_{13}\right) F_{22}\left(F_{31}\right) \\
& F_{13}^{2} F_{31}^{2}+F_{12}^{2} F_{23} F_{41}-F_{13}^{2} F_{32} F_{41}+F_{14} F_{21}^{2} F_{32}-F_{14} F_{23} F_{31}^{2}+F_{14} F_{23} F_{32} F_{41}= \\
& =\phi_{4}^{(0)}-F_{11}^{2} F_{22}^{2}-F_{11}^{2}\left(F_{23} F_{32}\right)-\left(F_{14}\right) F_{22}^{2}\left(F_{41}\right)+2 F_{11}\left(F_{12} F_{21}\right) F_{22}+2 F_{11} F_{12}\left(F_{23} F_{31}\right)+ \\
& +2 F_{11}\left(F_{13} F_{21} F_{32}\right)-2 F_{11}\left(F_{13}\right) F_{22}\left(F_{31}\right)+2\left(F_{12} F_{13}\right) F_{22}\left(F_{41}\right)+2\left(F_{14} F_{21}\right) F_{22}\left(F_{31}\right)- \\
& -\left(F_{14}\right) F_{23} F_{32}\left(F_{41}\right)
\end{aligned}
$$

Exemplo 4.3.5. Vamos calcular $\mathfrak{G}_{\mathfrak{s p}_{6}}$.

- $m=2$ (coeficientes em $\mathfrak{s p}_{2}$ ):

Pelo exemplo 4.0.1:

$$
\phi_{2}^{(2)}=-F_{11}^{2}-F_{12} F_{21}
$$


Mas, como $m=2$ e $\mathfrak{s p}_{2}$ é vista como a subálgebra de $\mathfrak{s p}_{6}$ gerada por $F_{i, j}$ com $i, j \in\{3,4\}$, devemos trocar 1 por 3 e 2 por 4. Assim temos:

$$
\begin{aligned}
& \phi_{2}^{(2)}=-F_{33}^{2}-F_{34} F_{43} \\
& \partial_{h(2)} \phi_{2}^{(2)}=\partial_{h(33)}\left(-F_{33}^{2}-F_{34} F_{43}\right)=-2 F_{33}
\end{aligned}
$$

- $m=1$ (coeficientes em $\mathfrak{s p}_{4}$ ):

Pelo exemplo 4.0.2:

$$
\begin{aligned}
\phi_{2}^{(1)} & =-F_{11}^{2}-F_{22}^{2}-2 F_{12} F_{21}-2 F_{13} F_{31}-F_{23} F_{32}-F_{14} F_{41} \\
\phi_{4}^{(1)} & =F_{11}^{2} F_{22}^{2}+F_{12}^{2} F_{21}^{2}+F_{13}^{2} F_{31}^{2}+F_{11}^{2} F_{23} F_{32}+F_{12}^{2} F_{23} F_{41}-F_{13}^{2} F_{32} F_{41}+F_{14} F_{21}^{2} F_{32}+ \\
& +F_{14} F_{22}^{2} F_{41}-F_{14} F_{23} F_{31}^{2}-2 F_{11} F_{12} F_{21} F_{22}-2 F_{11} F_{12} F_{23} F_{31}-2 F_{11} F_{13} F_{21} F_{32}+ \\
& +2 F_{11} F_{13} F_{22} F_{31}+2 F_{12} F_{13} F_{21} F_{31}-2 F_{12} F_{13} F_{22} F_{41}-2 F_{14} F_{21} F_{22} F_{31}+F_{14} F_{23} F_{32} F_{41}
\end{aligned}
$$

Mas, como $m=1$ e $\mathfrak{s p}_{4}$ é vista como a subálgebra de $\mathfrak{s p}_{6}$ gerada por $F_{i, j}$ com $i, j \in\{2,5\}$, devemos trocar 1 por 2, 2 por 3, 3 por 4 e 4 por 5. Assim temos:

$$
\begin{aligned}
\phi_{2}^{(1)} & =-F_{22}^{2}-F_{33}^{2}-2 F_{23} F_{32}-2 F_{24} F_{42}-F_{34} F_{43}-F_{25} F_{52} \\
\phi_{4}^{(1)} & =F_{22}^{2} F_{33}^{2}+F_{23}^{2} F_{32}^{2}+F_{24}^{2} F_{42}^{2}+F_{22}^{2} F_{34} F_{43}+F_{23}^{2} F_{34} F_{52}-F_{24}^{2} F_{43} F_{52}+F_{25} F_{32}^{2} F_{43}+ \\
& +F_{25} F_{33}^{2} F_{52}-F_{25} F_{34} F_{42}^{2}-2 F_{22} F_{23} F_{32} F_{33}-2 F_{22} F_{23} F_{34} F_{42}-2 F_{22} F_{24} F_{32} F_{43}+ \\
& +2 F_{22} F_{24} F_{33} F_{42}+2 F_{23} F_{24} F_{32} F_{42}-2 F_{23} F_{24} F_{33} F_{52}-2 F_{25} F_{32} F_{33} F_{42}+F_{25} F_{34} F_{43} F_{52}
\end{aligned}
$$

E:

$$
\begin{aligned}
& \partial_{h(1)} \phi_{2}^{(1)}=\partial_{F_{22}}\left(-F_{22}^{2}-F_{33}^{2}-2 F_{23} F_{32}-2 F_{24} F_{42}-F_{34} F_{43}-F_{25} F_{52}\right)=-2 F_{22} \\
& \partial_{h(1)} \phi_{4}^{(1)}=\partial_{F_{22}}\left(F_{22}^{2} F_{33}^{2}+F_{23}^{2} F_{32}^{2}+F_{24}^{2} F_{42}^{2}+F_{22}^{2} F_{34} F_{43}+F_{23}^{2} F_{34} F_{52}-F_{24}^{2} F_{43} F_{52}+F_{25} F_{32}^{2} F_{43}+\right. \\
& +F_{25} F_{33}^{2} F_{52}-F_{25} F_{34} F_{42}^{2}-2 F_{22} F_{23} F_{32} F_{33}-2 F_{22} F_{23} F_{34} F_{42}-2 F_{22} F_{24} F_{32} F_{43}+ \\
& \left.+2 F_{22} F_{24} F_{33} F_{42}+2 F_{23} F_{24} F_{32} F_{42}-2 F_{23} F_{24} F_{33} F_{52}-2 F_{25} F_{32} F_{33} F_{42}+F_{25} F_{34} F_{43} F_{52}\right) \\
& =2 F_{22} F_{33}^{2}+2 F_{22} F_{34} F_{43}-2 F_{23} F_{32} F_{33}-2 F_{23} F_{34} F_{42}-2 F_{24} F_{32} F_{43}+2 F_{24} F_{33} F_{42}
\end{aligned}
$$

- $m=0$ (coeficientes em $\mathfrak{s p}_{6}$ ):

Vamos primeiro calcular $\phi_{2}^{(0)}$. Esse é o coeficiente que acompanha $u^{4}$ no polinômio característico. Considere F a matriz do exemplo 4.0.3. Usando um programa para calcular determinante, temos:

$$
\begin{aligned}
\phi_{2}^{(0)} & =-F_{11}^{2}-2 F_{12} F_{21}-F_{22}^{2}-2 F_{13} F_{31}-2 F_{23} F_{32}-F_{33}^{2}-2 F_{14} F_{41}-2 F_{24} F_{42}-F_{34} F_{43}- \\
& -2 F_{15} F_{51}-F_{25} F_{52}-F_{16} F_{61}
\end{aligned}
$$

$E$ :

$$
\begin{aligned}
\partial_{h(0)} \phi_{2}^{(0)} & =\partial_{F_{11}}\left(-F_{11}^{2}-2 F_{12} F_{21}-F_{22}^{2}-2 F_{13} F_{31}-2 F_{23} F_{32}-F_{33}^{2}-\right. \\
& \left.-2 F_{14} F_{41}-2 F_{24} F_{42}-F_{34} F_{43}-2 F_{15} F_{51}-F_{25} F_{52}-F_{16} F_{61}\right) \\
& =-2 F_{11}
\end{aligned}
$$


Pelo que já fizemos, temos os seguintes geradores:

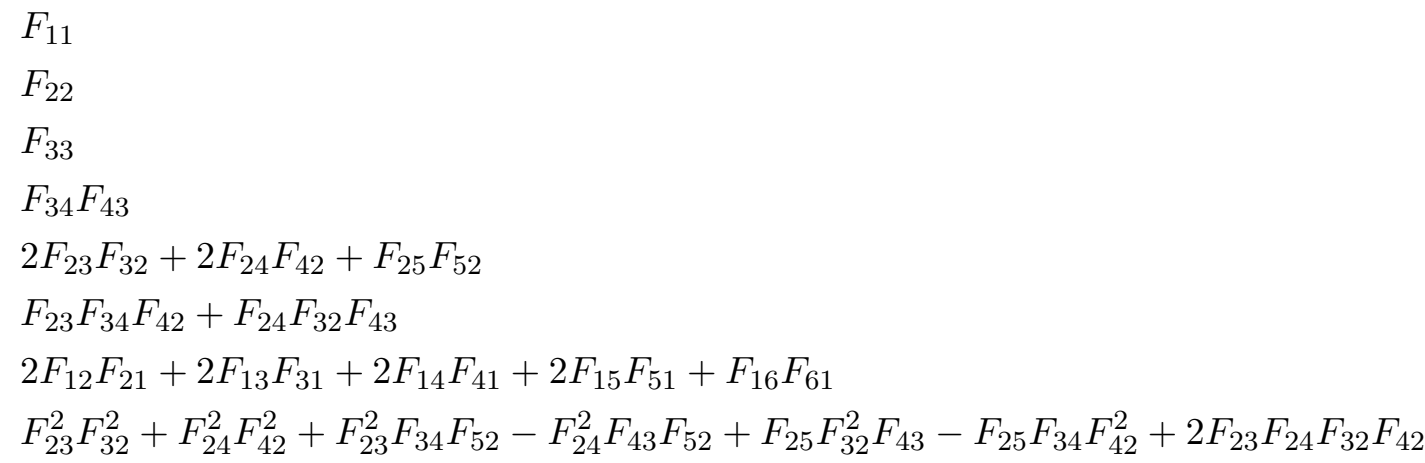

pela definição de variedade e pelas seguintes igualdades:

$$
\begin{aligned}
& F_{11}=-\frac{1}{2} \partial_{h(0)} \phi_{2}^{(0)} \\
& F_{22}=-\frac{1}{2} \partial_{h(1)} \phi_{2}^{(1)} \\
& F_{33}=-\frac{1}{2} \partial_{h(2)} \phi_{2}^{(2)} \\
& \left.F_{34} F_{43}=-\phi_{2}^{(} 2\right)-F_{33}^{2} \\
& 2 F_{23} F_{32}+2 F_{24} F_{42}+F_{25} F_{52}=-\phi_{2}^{(1)}-F_{22}^{2}-F_{33}^{2}-F_{34} F_{43} \\
& F_{23} F_{34} F_{42}+F_{24} F_{32} F_{43}=-\frac{1}{2} \partial_{h(1)} \phi_{4}^{(1)}+F_{22}^{2} F_{33}+F_{22}\left(F_{34} F_{43}\right)-\left(F_{23} F_{32}\right) F_{33}+F_{24}\left(F_{33}\right) F_{42} \\
& 2 F_{12} F_{21}+2 F_{13} F_{31}+2 F_{14} F_{41}+2 F_{15} F_{51}+F_{16} F_{61}= \\
& =-\phi_{2}^{(0)}-F_{11}^{2}-F_{22}^{2}-\left(2 F_{23} F_{32}+2 F_{24} F_{42}+F_{25} F_{52}\right)-F_{33}^{2}-F_{34} F_{43} \\
& F_{23}^{2} F_{32}^{2}+F_{24}^{2} F_{42}^{2}+F_{23}^{2} F_{34} F_{52}-F_{24}^{2} F_{43} F_{52}+F_{25} F_{32}^{2} F_{43}-F_{25} F_{34} F_{42}^{2}+2 F_{23} F_{24} F_{32} F_{42}= \\
& =\phi_{4}^{(1)}-F_{22}^{2} F_{33}^{2}-F_{22}^{2} F_{34} F_{43}-F_{25} F_{33}^{2} F_{52}+2 F_{22} F_{23} F_{32} F_{33}+2 F_{22} F_{23} F_{34} F_{42}+ \\
& +2 F_{22} F_{24} F_{32} F_{43}-2 F_{22} F_{24} F_{33} F_{42}+2 F_{23} F_{24} F_{33} F_{52}+2 F_{25} F_{32} F_{33} F_{42}-\left(F_{25}\right) F_{34} F_{43}\left(F_{52}\right)
\end{aligned}
$$

Pela fórmula do determinante e por $\phi_{6}^{(0)}$ ser o coeficiente independente (que não acompanha a variável u), temos:

$$
\left.\begin{array}{rl}
\phi_{6}^{(0)}=\operatorname{det}\left(\begin{array}{cccccc}
F_{11} & F_{12} & F_{13} & F_{14} & F_{15} & F_{16} \\
F_{21} & F_{22} & F_{23} & F_{24} & F_{25} & F_{15} \\
F_{31} & F_{32} & F_{33} & F_{34} & F_{24} & F_{14} \\
F_{41} & F_{42} & F_{43} & -F_{33} & -F_{23} & -F_{13} \\
F_{51} & F_{52} & F_{42} & -F_{32} & -F_{22} & -F_{12} \\
F_{61} & F_{51} & F_{41} & -F_{31} & -F_{21} & -F_{11}
\end{array}\right) \\
\partial_{F_{11}} \phi_{6}^{(0)}=\operatorname{det}\left(\begin{array}{cccccc}
F_{22} & F_{23} & F_{24} & F_{25} & F_{15} \\
F_{32} & F_{33} & F_{34} & F_{24} & F_{14} \\
F_{42} & F_{43} & -F_{33} & -F_{23} & -F_{13} \\
F_{52} & F_{42} & -F_{32} & -F_{22} & -F_{12} \\
F_{51} & F_{41} & -F_{31} & -F_{21} & -F_{11}
\end{array}\right)
\end{array}\right) \quad\left(\begin{array}{ccccccc}
F_{11} & F_{12} & F_{13} & F_{14} & F_{15} \\
F_{21} & F_{22} & F_{23} & F_{24} & F_{25} \\
F_{31} & F_{32} & F_{33} & F_{34} & F_{24} \\
F_{41} & F_{42} & F_{43} & -F_{33} & -F_{23} \\
F_{51} & F_{52} & F_{42} & -F_{32} & -F_{22}
\end{array}\right)
$$

Os outros dois geradores são $\psi_{4}^{(0)}$ e $\partial_{F_{11}} \psi_{4}^{(0)}$.

Conjectura 4.3.1. A variedade de Gelfand-Tsetlin $\mathfrak{G}_{\mathfrak{s p}_{2 n}}$ é equidimensional com dimensão:

$$
\operatorname{dim}_{\mathfrak{s p}_{2 n}}=n(2 n+1)-2 \frac{n(n+1)}{2}=n^{2} .
$$


Para $\mathfrak{G}_{\mathfrak{s p}_{4}}$, provamos que a conjectura acima é verdadeira:

Teorema 4.3.1. A variedade de Gelfand-Tsetlin $\mathfrak{G}_{\mathfrak{s p}_{4}}$ é equidimensional com dimensão 4.

Demonstração. No exemplo 4.0.4. calculamos os geradores de $\mathfrak{G}_{\mathfrak{s p}_{4}} \subset k^{10}$. Usando uma notação mais tradicional, temos que os geradores são:

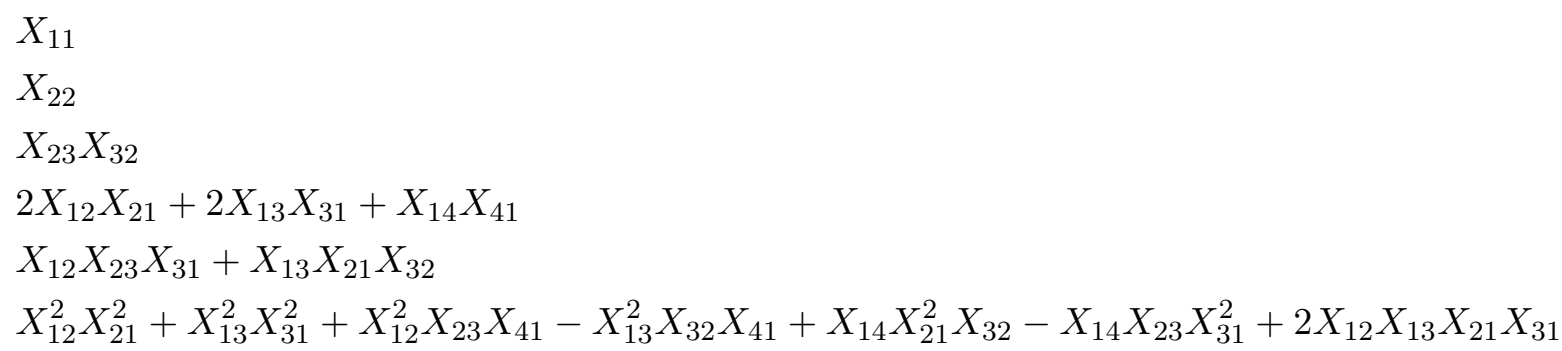

Sejam

$$
V=\mathcal{V}\left(X_{11}, X_{22}, X_{23}, 2 X_{12} X_{21}+2 X_{13} X_{31}+X_{14} X_{41}, X_{13} X_{21} X_{32}, A\right)
$$

onde $A=X_{12}^{2} X_{21}^{2}+X_{13}^{2} X_{31}^{2}-X_{13}^{2} X_{32} X_{41}+X_{14} X_{21}^{2} X_{32}+2 X_{12} X_{13} X_{21} X_{31}$, e

$$
U=\mathcal{V}\left(X_{11}, X_{22}, X_{32}, 2 X_{12} X_{21}+2 X_{13} X_{31}+X_{14} X_{41}, X_{12} X_{23} X_{31}, B\right)
$$

onde $B=X_{12}^{2} X_{21}^{2}+X_{13}^{2} X_{31}^{2}+X_{12}^{2} X_{23} X_{41}-X_{14} X_{23} X_{31}^{2}+2 X_{12} X_{13} X_{21} X_{31}$. Observe que $\mathfrak{G}_{\mathfrak{s p}_{4}}=V \cup U$ e que $V \cong U$, pois os geradores de $U$ são a imagem dos geradores de $V$ pela função que leva $X_{i j}$ em $X_{j i}$. Assim, basta provar que $V$ é equidimensional de dimensão 4 .

Agora considere

$$
\begin{aligned}
& V_{1}=\mathcal{V}\left(X_{11}, X_{22}, X_{23}, X_{13}, 2 X_{12} X_{21}+X_{14} X_{41}, X_{12}^{2} X_{21}^{2}+X_{14} X_{21}^{2} X_{32}\right) \\
& V_{2}=\mathcal{V}\left(X_{11}, X_{22}, X_{23}, X_{21}, 2 X_{13} X_{31}+X_{14} X_{41}, X_{13}^{2} X_{31}^{2}-X_{13}^{2} X_{32} X_{41}\right) \\
& V_{3}=\mathcal{V}\left(X_{11}, X_{22}, X_{23}, X_{32}, 2 X_{12} X_{21}+2 X_{13} X_{31}+X_{14} X_{41}, X_{12}^{2} X_{21}^{2}+X_{13}^{2} X_{31}^{2}+2 X_{12} X_{13} X_{21} X_{31}\right)
\end{aligned}
$$

Primeiro provaremos que $V_{1}$ é equidimensional de dimensão 4 . Temos:

$$
\begin{aligned}
& V_{1}=\mathcal{V}\left(X_{11}, X_{22}, X_{23}, X_{13}, 2 X_{12} X_{21}+X_{14} X_{41}, X_{12}^{2} X_{21}^{2}+X_{14} X_{21}^{2} X_{32}\right)= \\
& =\mathcal{V}\left(X_{11}, X_{22}, X_{23}, X_{13}, 2 X_{12} X_{21}+X_{14} X_{41}, X_{21}^{2}\left(X_{12}^{2}+X_{14} X_{32}\right)\right)= \\
& =\mathcal{V}\left(X_{11}, X_{22}, X_{23}, X_{13}, X_{21}, X_{14} X_{41}\right) \cup \mathcal{V}\left(X_{11}, X_{22}, X_{23}, X_{13}, 2 X_{12} X_{21}+X_{14} X_{41}, X_{12}^{2}+X_{14} X_{32}\right)= \\
& =\mathcal{V}\left(X_{11}, X_{22}, X_{23}, X_{13}, X_{21}, X_{14}\right) \cup \mathcal{V}\left(X_{11}, X_{22}, X_{23}, X_{13}, X_{21}, X_{41}\right) \cup \\
& \cup \mathcal{V}\left(X_{11}, X_{22}, X_{23}, X_{13}, 2 X_{12} X_{21}+X_{14} X_{41}, X_{12}^{2}+X_{14} X_{32}\right)
\end{aligned}
$$

Como $\mathcal{V}\left(X_{11}, X_{22}, X_{23}, X_{13}, X_{21}, X_{14}\right)$ e $\mathcal{V}\left(X_{11}, X_{22}, X_{23}, X_{13}, X_{21}, X_{41}\right)$ são equidimensionais de dimensão $10-6=4$, basta provarmos que $\mathcal{V}\left(X_{11}, X_{22}, X_{23}, X_{13}, 2 X_{12} X_{21}+X_{14} X_{41}, X_{12}^{2}+X_{14} X_{32}\right)$ é equidimensional de dimensão 4 . Note que:

$$
\begin{aligned}
& \mathcal{V}\left(X_{11}, X_{22}, X_{23}, X_{13}, 2 X_{12} X_{21}+X_{14} X_{41}, X_{12}^{2}+X_{14} X_{32}, X_{32}\right)= \\
& =\mathcal{V}\left(X_{11}, X_{22}, X_{23}, X_{13}, X_{32}, 2 X_{12} X_{21}+X_{14} X_{41}, X_{12}^{2}\right)= \\
& =\mathcal{V}\left(X_{11}, X_{22}, X_{23}, X_{13}, X_{32}, X_{12}, X_{14} X_{41}\right)= \\
& =\mathcal{V}\left(X_{11}, X_{22}, X_{23}, X_{13}, X_{32}, X_{12}, X_{14}\right) \cup \mathcal{V}\left(X_{11}, X_{22}, X_{23}, X_{13}, X_{32}, X_{12}, X_{41}\right)
\end{aligned}
$$

é equidimensional de dimensão 10-7=3. Então $\mathcal{V}\left(X_{11}, X_{22}, X_{23}, X_{13}, 2 X_{12} X_{21}+X_{14} X_{41}, X_{12}^{2}+\right.$ $\left.X_{14} X_{32}\right)$ é equidimensional de dimensão 4 . 
Vamos agora provar que $V_{2}$ é equidimensional de dimensão 4. Temos:

$$
\begin{aligned}
& V_{2}=\mathcal{V}\left(X_{11}, X_{22}, X_{23}, X_{21}, 2 X_{13} X_{31}+X_{14} X_{41}, X_{13}^{2} X_{31}^{2}-X_{13}^{2} X_{32} X_{41}\right) \\
& =\mathcal{V}\left(X_{11}, X_{22}, X_{23}, X_{21}, 2 X_{13} X_{31}+X_{14} X_{41}, X_{13}^{2}\left(X_{31}^{2}-X_{32} X_{41}\right)\right)= \\
& =\mathcal{V}\left(X_{11}, X_{22}, X_{23}, X_{21} X_{13}, X_{14}\right) \cup \mathcal{V}\left(X_{11}, X_{22}, X_{23}, X_{21} X_{13}, X_{41}\right) \cup \\
& \cup \mathcal{V}\left(X_{11}, X_{22}, X_{23}, X_{21}, 2 X_{13} X_{31}+X_{14} X_{41}, X_{31}^{2}-X_{32} X_{41}\right)
\end{aligned}
$$

Então, basta provar que $\mathcal{V}\left(X_{11}, X_{22}, X_{23}, X_{21}, 2 X_{13} X_{31}+X_{14} X_{41}, X_{31}^{2}-X_{32} X_{41}\right)$ é equidimensal de dimensão 4. Mas

$$
\begin{aligned}
& \mathcal{V}\left(X_{11}, X_{22}, X_{23}, X_{21}, 2 X_{13} X_{31}+X_{14} X_{41}, X_{31}^{2}-X_{32} X_{41}, X_{32}\right)= \\
& =\mathcal{V}\left(X_{11}, X_{22}, X_{23}, X_{21}, X_{32}, 2 X_{13} X_{31}+X_{14} X_{41}, X_{31}^{2}\right)= \\
& =\mathcal{V}\left(X_{11}, X_{22}, X_{23}, X_{21}, X_{32}, X_{31}, X_{14} X_{41}\right)= \\
& =\mathcal{V}\left(X_{11}, X_{22}, X_{23}, X_{21}, X_{32}, X_{31}, X_{14}\right) \cup \mathcal{V}\left(X_{11}, X_{22}, X_{23}, X_{21}, X_{32}, X_{31}, X_{41}\right)
\end{aligned}
$$

é equidimensional de dimensão $10-7=3$. Portanto, $V_{2}$ também é equidimensional.

Por último, vamos analisar $V_{3}$. Temos que:

$\mathcal{V}\left(X_{11}, X_{22}, X_{23}, X_{32}, 2 X_{12} X_{21}+2 X_{13} X_{31}+X_{14} X_{41}, X_{12}^{2} X_{21}^{2}+X_{13}^{2} X_{31}^{2}+2 X_{12} X_{13} X_{21} X_{31}, X_{12}\right)=$ $=\mathcal{V}\left(X_{11}, X_{22}, X_{23}, X_{32}, X_{12}, 2 X_{13} X_{31}+X_{14} X_{41}, X_{13}^{2} X_{31}^{2}\right)=$

$=\mathcal{V}\left(X_{11}, X_{22}, X_{23}, X_{32}, X_{12}, X_{14} X_{41}, X_{13} X_{31}\right)=$

$=\mathcal{V}\left(X_{11}, X_{22}, X_{23}, X_{32}, X_{12}, X_{14}, X_{13} X_{31}\right) \cup \mathcal{V}\left(X_{11}, X_{22}, X_{23}, X_{32}, X_{12} X_{41}, X_{13} X_{31}\right)=$

$=\mathcal{V}\left(X_{11}, X_{22}, X_{23}, X_{12}, X_{32}, X_{14}, X_{13}\right) \cup \mathcal{V}\left(X_{11}, X_{22}, X_{23}, X_{32}, X_{12}, X_{14}, X_{31}\right) \cup$

$\cup \mathcal{V}\left(X_{11}, X_{22}, X_{23}, X_{12}, X_{32}, X_{41}, X_{13}\right) \cup \mathcal{V}\left(X_{11}, X_{22}, X_{23}, X_{32}, X_{12}, X_{41}, X_{31}\right)$

é equidimensional de dimensão $10-7=3$. Então $V_{3}$ é equidimensional de dimensão 4 .

Portanto $\mathfrak{G}_{\mathfrak{s p}_{4}}$ é equidimensional de dimensão 4 .

Para $\mathfrak{G}_{\mathfrak{s p}_{6}}$, descubrimos e demonstramos o seguinte resultado:

Teorema 4.3.2. A variedade de Gelfand-Tsetlin $\mathfrak{G}_{\mathfrak{s p}_{6}}$ é equidimensional de dimenão 9 se e somente se a variedade

$$
\mathcal{V}\left(X_{11}, X_{22}, X_{33}, X_{34}, X_{24}, 2 X_{23} X_{32}+X_{25} X_{52}, A, X_{23}^{2}+X_{25} X_{43}, \phi_{6}^{(0)}, \partial_{X_{11}} \phi_{6}^{(0)}, \phi_{4}^{(0)}, \partial_{X_{11}} \phi_{4}^{(0)}\right)
$$

onde $A=2 X_{12} X_{21}+2 X_{13} X_{31}+2 X_{14} X_{41}+2 X_{15} X_{51}+X_{16} X_{61}$, é equidimensional de dimensão 9.

Demonstração. No exemplo 4.0.5. vimos que os geradores de $\mathfrak{G}_{\mathfrak{s p}_{6}}$, usando uma notação mais tra- 
dicional, são:

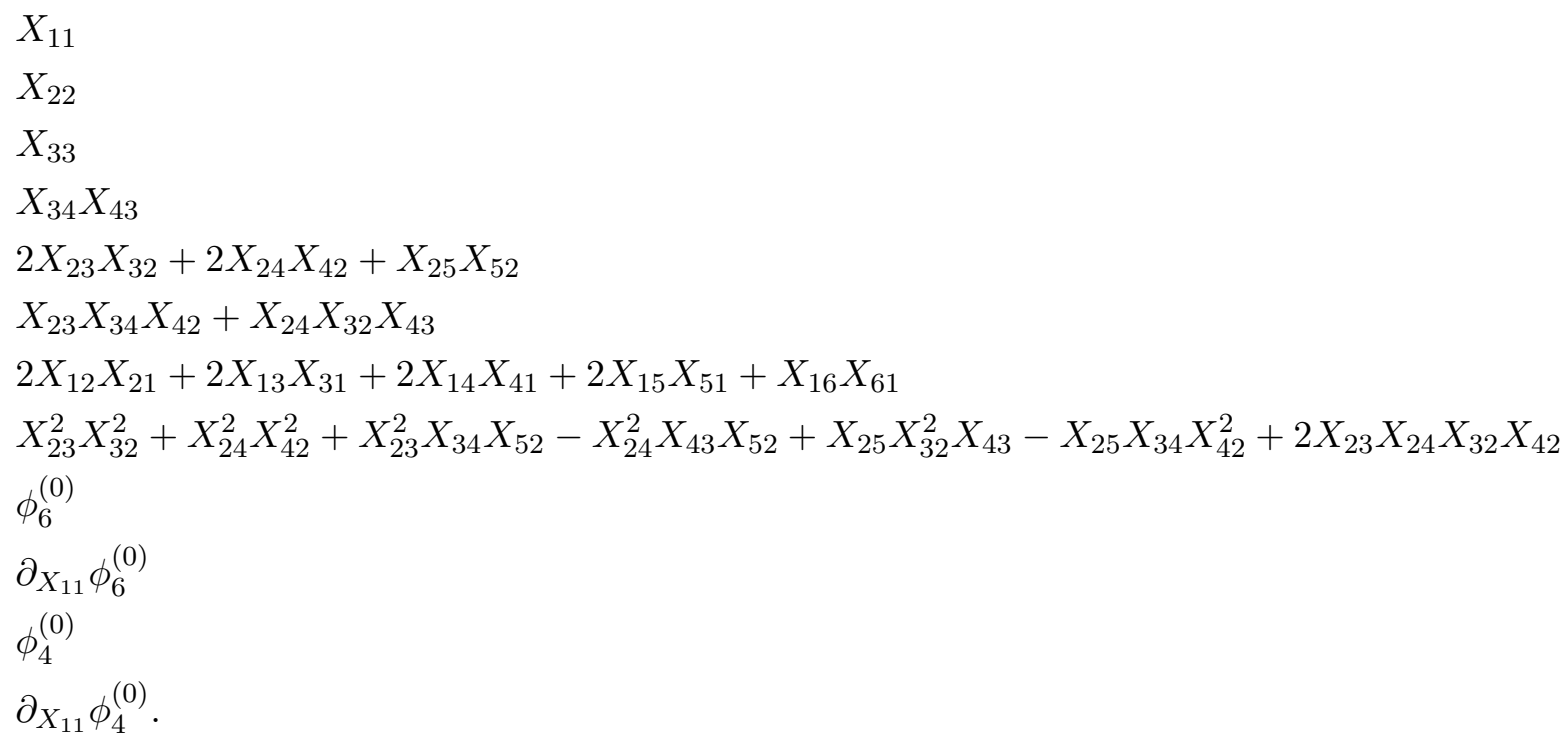

Sejam

$$
\begin{aligned}
V= & \mathcal{V}\left(X_{11}, X_{22}, X_{33}, X_{34}, 2 X_{23} X_{32}+2 X_{24} X_{42}+X_{25} X_{52}, X_{24} X_{32} X_{43}, A, B, \phi_{6}^{(0)}, \partial_{X_{11}} \phi_{6}^{(0)}, \phi_{4}^{(0)},\right. \\
& \left.\partial_{X_{11}} \phi_{4}^{(0)}\right)
\end{aligned}
$$

onde $A=2 X_{12} X_{21}+2 X_{13} X_{31}+2 X_{14} X_{41}+2 X_{15} X_{51}+X_{16} X_{61} \mathrm{e}$ $B=X_{23}^{2} X_{32}^{2}+X_{24}^{2} X_{42}^{2}-X_{24}^{2} X_{43} X_{52}+X_{25} X_{32}^{2} X_{43}+2 X_{23} X_{24} X_{32} X_{42}$, e:

$$
\begin{aligned}
U= & \mathcal{V}\left(X_{11}, X_{22}, X_{33}, X_{43}, 2 X_{23} X_{32}+2 X_{24} X_{42}+X_{25} X_{52}, X_{23} X_{34} X_{42}, A, C, \phi_{6}^{(0)}, \partial_{X_{11}} \phi_{6}^{(0)}, \phi_{4}^{(0)},\right. \\
& \left.\partial_{X_{11}} \phi_{4}^{(0)}\right)
\end{aligned}
$$

onde $C=X_{23}^{2} X_{32}^{2}+X_{24}^{2} X_{42}^{2}+X_{23}^{2} X_{34} X_{52}-X_{25} X_{34} X_{42}^{2}+2 X_{23} X_{24} X_{32} X_{42}$

Como o determinante de uma matriz é igual ao determinante da sua transposta, temos que o automorfismo $f$ do anel de polinômios nas 21 variáveis $X_{i j}$, que leva $X_{i j}$ em $X_{j i}$, leva $\phi_{4}^{(0)}, \phi_{6}^{(0)}$ e suas diferenciais neles mesmos. Com isso concluimos que $V \cong U$. Assim, só precisamos provar que $V$ é equidimensional de dimensão 9 .

Defina as seguintes variedades

$V_{1}=$

$\mathcal{V}\left(X_{11}, X_{22}, X_{33}, X_{34}, X_{24}, 2 X_{23} X_{32}+X_{25} X_{52}, A, X_{23}^{2} X_{32}^{2}+X_{25} X_{32}^{2} X_{43}, \phi_{6}^{(0)}, \partial_{X_{11}} \phi_{6}^{(0)}, \phi_{4}^{(0)}, \partial_{X_{11}} \phi_{4}^{(0)}\right)$ $V_{2}=$

$\mathcal{V}\left(X_{11}, X_{22}, X_{33}, X_{34}, X_{32}, 2 X_{24} X_{42}+X_{25} X_{52}, A, X_{24}^{2} X_{42}^{2}-X_{24}^{2} X_{43} X_{52}, \phi_{6}^{(0)}, \partial_{X_{11}} \phi_{6}^{(0)}, \phi_{4}^{(0)}, \partial_{X_{11}} \phi_{4}^{(0)}\right)$ $V_{3}=$

$\mathcal{V}\left(X_{11}, X_{22}, X_{33}, X_{34}, X_{43}, 2 X_{23} X_{32}+2 X_{24} X_{42}+X_{25} X_{52}, A, X_{23}^{2} X_{32}^{2}+X_{24}^{2} X_{42}^{2}+2 X_{23} X_{24} X_{32} X_{42}\right.$, $\left.\phi_{6}^{(0)}, \partial_{X_{11}} \phi_{6}^{(0)}, \phi_{4}^{(0)}, \partial_{X_{11}} \phi_{4}^{(0)}\right)$

Temos: $V=V_{1} \cup V_{2} \cup V_{3}$. 
Para $V_{1}$ temos:

$$
\begin{aligned}
& V_{1}=\mathcal{V}\left(X_{11}, X_{22}, X_{33}, X_{34}, X_{24}, 2 X_{23} X_{32}+X_{25} X_{52}, A, X_{32}^{2}, \phi_{6}^{(0)}, \partial_{X_{11}} \phi_{6}^{(0)}, \phi_{4}^{(0)}, \partial_{X_{11}} \phi_{4}^{(0)}\right) \cup \\
& \cup \mathcal{V}\left(X_{11}, X_{22}, X_{33}, X_{34}, X_{24}, 2 X_{23} X_{32}+X_{25} X_{52}, A, X_{23}^{2}+X_{25} X_{43}, \phi_{6}^{(0)}, \partial_{X_{11}} \phi_{6}^{(0)}, \phi_{4}^{(0)}, \partial_{X_{11}} \phi_{4}^{(0)}\right)= \\
& =\mathcal{V}\left(X_{11}, X_{22}, X_{33}, X_{34}, X_{24}, X_{32}, X_{25} X_{52}, A, \phi_{6}^{(0)}, \partial_{X_{11}} \phi_{6}^{(0)}, \phi_{4}^{(0)}, \partial_{X_{11}} \phi_{4}^{(0)}\right) \cup \\
& \cup \mathcal{V}\left(X_{11}, X_{22}, X_{33}, X_{34}, X_{24}, 2 X_{23} X_{32}+X_{25} X_{52}, A, X_{23}^{2}+X_{25} X_{43}, \phi_{6}^{(0)}, \partial_{X_{11}} \phi_{6}^{(0)}, \phi_{4}^{(0)}, \partial_{X_{11}} \phi_{4}^{(0)}\right)
\end{aligned}
$$

\section{Defina:}

$W_{1}=\mathcal{V}\left(X_{11}, X_{22}, X_{33}, X_{34}, X_{24}, X_{32}, X_{25}, A, \phi_{6}^{(0)}, \partial_{X_{11}} \phi_{6}^{(0)}, \phi_{4}^{(0)}, \partial_{X_{11}} \phi_{4}^{(0)}\right)$

$W_{2}=\mathcal{V}\left(X_{11}, X_{22}, X_{33}, X_{34}, X_{24}, X_{32}, X_{52}, A, \phi_{6}^{(0)}, \partial_{X_{11}} \phi_{6}^{(0)}, \phi_{4}^{(0)}, \partial_{X_{11}} \phi_{4}^{(0)}\right)$

$W_{3}=\mathcal{V}\left(X_{11}, X_{22}, X_{33}, X_{34}, X_{24}, 2 X_{23} X_{32}+X_{25} X_{52}, A, X_{23}^{2}+X_{25} X_{43}, \phi_{6}^{(0)}, \partial_{X_{11}} \phi_{6}^{(0)}, \phi_{4}^{(0)}, \partial_{X_{11}} \phi_{4}^{(0)}\right)$.

Dessa forma, $V_{1}=W_{1} \cup W_{2} \cup W_{3}$.

Agora para $V_{2}$ temos:

$$
\begin{aligned}
& V_{2}=\mathcal{V}\left(X_{11}, X_{22}, X_{33}, X_{34}, X_{32}, 2 X_{24} X_{42}+X_{25} X_{52}, A, X_{24}^{2}, \phi_{6}^{(0)}, \partial_{X_{11}} \phi_{6}^{(0)}, \phi_{4}^{(0)}, \partial_{X_{11}} \phi_{4}^{(0)}\right) \cup \\
& \cup \mathcal{V}\left(X_{11}, X_{22}, X_{33}, X_{34}, X_{32}, 2 X_{24} X_{42}+X_{25} X_{52}, A, X_{42}^{2}-X_{43} X_{52}, \phi_{6}^{(0)}, \partial_{X_{11}} \phi_{6}^{(0)}, \phi_{4}^{(0)}, \partial_{X_{11}} \phi_{4}^{(0)}\right)= \\
& =\mathcal{V}\left(X_{11}, X_{22}, X_{33}, X_{34}, X_{32}, X_{24}, X_{25} X_{52}, A, \phi_{6}^{(0)}, \partial_{X_{11}} \phi_{6}^{(0)}, \phi_{4}^{(0)}, \partial_{X_{11}} \phi_{4}^{(0)}\right) \cup \\
& \cup \mathcal{V}\left(X_{11}, X_{22}, X_{33}, X_{34}, X_{32}, 2 X_{24} X_{42}+X_{25} X_{52}, A, X_{42}^{2}-X_{43} X_{52}, \phi_{6}^{(0)}, \partial_{X_{11}} \phi_{6}^{(0)}, \phi_{4}^{(0)}, \partial_{X_{11}} \phi_{4}^{(0)}\right)
\end{aligned}
$$

Defina:

$W_{4}=\mathcal{V}\left(X_{11}, X_{22}, X_{33}, X_{34}, X_{32}, 2 X_{24} X_{42}+X_{25} X_{52}, A, X_{42}^{2}-X_{43} X_{52}, \phi_{6}^{(0)}, \partial_{X_{11}} \phi_{6}^{(0)}, \phi_{4}^{(0)}, \partial_{X_{11}} \phi_{4}^{(0)}\right)$

Dessa forma, $V_{2}=W_{1} \cup W_{2} \cup W_{4}$.

Para $V_{3}$ temos:

$$
\begin{aligned}
& V_{3}=\mathcal{V}\left(X_{11}, X_{22}, X_{33}, X_{34}, X_{43}, 2 X_{23} X_{32}+2 X_{24} X_{42}+X_{25} X_{52}, A,\left(X_{23} X_{32}+X_{24} X_{42}\right)^{2},\right. \\
& \left.\phi_{6}^{(0)}, \partial_{X_{11}} \phi_{6}^{(0)}, \phi_{4}^{(0)}, \partial_{X_{11}} \phi_{4}^{(0)}\right)= \\
& =\mathcal{V}\left(X_{11}, X_{22}, X_{33}, X_{34}, X_{43}, X_{23} X_{32}+X_{24} X_{42}, X_{25} X_{52}, A, \phi_{6}^{(0)}, \partial_{X_{11}} \phi_{6}^{(0)}, \phi_{4}^{(0)}, \partial_{X_{11}} \phi_{4}^{(0)}\right)
\end{aligned}
$$

Defina:

$$
\begin{aligned}
& W_{5}=\mathcal{V}\left(X_{11}, X_{22}, X_{33}, X_{34}, X_{43}, X_{25}, X_{23} X_{32}+X_{24} X_{42}, A, \phi_{6}^{(0)}, \partial_{X_{11}} \phi_{6}^{(0)}, \phi_{4}^{(0)}, \partial_{X_{11}} \phi_{4}^{(0)}\right) \\
& W_{6}=\mathcal{V}\left(X_{11}, X_{22}, X_{33}, X_{34}, X_{43}, X_{52}, X_{23} X_{32}+X_{24} X_{42}, A, \phi_{6}^{(0)}, \partial_{X_{11}} \phi_{6}^{(0)}, \phi_{4}^{(0)}, \partial_{X_{11}} \phi_{4}^{(0)}\right)
\end{aligned}
$$

Assim, $V_{3}=W_{5} \cup W_{6}$.

Portanto, para provar que $\mathfrak{G}_{\mathfrak{s p}_{6}}$ é equidimensional de dimensão 9 , basta provar que $W_{1}, W_{2}$, $W_{3}, W_{4}, W_{5}$ e $W_{6}$ são equidimensionais de dimensão 9.

(i) $W_{1}$

$$
W_{1}=\mathcal{V}\left(X_{11}, X_{22}, X_{33}, X_{34}, X_{24}, X_{32}, X_{25}, A, \phi_{6}^{(0)}, \partial_{X_{11}} \phi_{6}^{(0)}, \phi_{4}^{(0)}, \partial_{X_{11}} \phi_{4}^{(0)}\right)
$$

Calculando o determinante da matriz $F$ do exemplo 4.3.3 para

$X_{22}=X_{33}=X_{34}=X_{24}=X_{32}=X_{25}=0$, temos (considerando $X_{11}=0$ ):

$$
\partial_{X_{11}} \phi_{6}^{(0)}=2 X_{14} X_{23}^{2} X_{31} X_{52}
$$


Assim, defina:

$$
\begin{aligned}
& Z_{1}^{(1)}=\mathcal{V}\left(X_{11}, X_{22}, X_{33}, X_{34}, X_{24}, X_{32}, X_{25}, X_{14}, A, \phi_{6}^{(0)}, \phi_{4}^{(0)}, \partial_{X_{11}} \phi_{4}^{(0)}\right) \\
& Z_{2}^{(1)}=\mathcal{V}\left(X_{11}, X_{22}, X_{33}, X_{34}, X_{24}, X_{32}, X_{25}, X_{23}, A, \phi_{6}^{(0)}, \phi_{4}^{(0)}, \partial_{X_{11}} \phi_{4}^{(0)}\right) \\
& Z_{3}^{(1)}=\mathcal{V}\left(X_{11}, X_{22}, X_{33}, X_{34}, X_{24}, X_{32}, X_{25}, X_{31}, A, \phi_{6}^{(0)}, \phi_{4}^{(0)}, \partial_{X_{11}} \phi_{4}^{(0)}\right) \\
& Z_{4}^{(1)}=\mathcal{V}\left(X_{11}, X_{22}, X_{33}, X_{34}, X_{24}, X_{32}, X_{25}, X_{52}, A, \phi_{6}^{(0)}, \phi_{4}^{(0)}, \partial_{X_{11}} \phi_{4}^{(0)}\right)
\end{aligned}
$$

obtendo $W_{1}=Z_{1}^{(1)} \cup Z_{2}^{(1)} \cup Z_{3}^{(1)} \cup Z_{4}^{(1)}$. Então para provar que $W_{1}$ é equidimensional de dimensão 9 basta provar que $Z_{1}^{(1)}, Z_{2}^{(1)}, Z_{3}^{(1)}$ e $Z_{4}^{(1)}$ são equidimensionais de dimensão 9.

(a) $Z_{1}^{(1)}$

Calculando o determinante da matriz $F$ do exemplo 4.3.3 para

$X_{22}=X_{33}=X_{34}=X_{24}=X_{32}=X_{25}=X_{14}=0$, temos:

$$
\begin{aligned}
\phi_{6}^{(0)} & =-X_{12}^{2} X_{23}^{2} X_{31}^{2}-2 X_{12} X_{15} X_{23} X_{31}^{2} X_{42}-X_{15}^{2} X_{31}^{2} X_{42}^{2}+ \\
& +2 X_{13} X_{15} X_{23} X_{31}^{2} X_{52}-X_{16} X_{23}^{2} X_{31}^{2} X_{52}+X_{15}^{2} X_{31}^{2} X_{43} X_{52}= \\
& =X_{31}^{2}\left[-\left(X_{12} X_{23}+X_{15} X_{42}\right)^{2}+X_{52}\left(2 X_{13} X_{15} X_{23}-X_{16} X_{23}^{2}+X_{15}^{2} X_{43}\right)\right] \\
\phi_{4}^{(0)} & =X_{12}^{2} X_{21}^{2}+2 X_{12} X_{13} X_{21} X_{31}+X_{13}^{2} X_{31}^{2}+2 X_{16} X_{21} X_{31} X_{42}+ \\
& +X_{16} X_{31}^{2} X_{43}+2 X_{12} X_{15} X_{21} X_{51}+2 X_{13} X_{15} X_{31} X_{51}-2 X_{16} X_{23} X_{31} X_{51}+ \\
& +X_{15}^{2} X_{51}^{2}+X_{16} X_{21}^{2} X_{52}-2 X_{15} X_{23} X_{31} X_{52}-X_{15}^{2} X_{52} X_{61}= \\
& =\left(X_{12} X_{21}+X_{13} X_{31}+X_{15} X_{51}\right)^{2}+2 X_{16} X_{21} X_{31} X_{42}+X_{16} X_{31}^{2} X_{43}- \\
& -2 X_{16} X_{23} X_{31} X_{51}+X_{16} X_{21}^{2} X_{52}-2 X_{15} X_{23} X_{31} X_{52}-X_{15}^{2} X_{52} X_{61} \\
\partial_{X_{11}} \phi_{4}^{(0)} & =-2 X_{12} X_{23} X_{31}-2 X_{15} X_{31} X_{42}-2 X_{15} X_{21} X_{52}
\end{aligned}
$$

Além disso, $A=2 X_{12} X_{21}+2 X_{13} X_{31}+2 X_{15} X_{51}+X_{16} X_{61}$.

Por $\phi_{6}^{(0)}$ temos que $Z_{1}^{(1)}$ é a união das duas variedades abaixo:

$$
\mathcal{V}\left(X_{11}, X_{22}, X_{33}, X_{34}, X_{24}, X_{32}, X_{25}, X_{14}, X_{31}, A, \phi_{4}^{(0)}, \partial_{X_{11}} \phi_{4}^{(0)}\right)
$$

e

$$
\begin{aligned}
& \mathcal{V}\left(X_{11}, X_{22}, X_{33}, X_{34}, X_{24}, X_{32}, X_{25}, X_{14}\right. \\
& \left.-\left(X_{12} X_{23}+X_{15} X_{42}\right)^{2}+X_{52}\left(2 X_{13} X_{15} X_{23}-X_{16} X_{23}^{2}+X_{15}^{2} X_{43}\right), A, \phi_{4}^{(0)}, \partial_{X_{11}} \phi_{4}^{(0)}\right)
\end{aligned}
$$


A variedade (4.7) é equidimensional de dimensão 9 pois é igual a:

$$
\begin{aligned}
& \mathcal{V}\left(X_{11}, X_{22}, X_{33}, X_{34}, X_{24}, X_{32}, X_{25}, X_{14}, X_{31}, A, \phi_{4}^{(0)}, \partial_{X_{11}} \phi_{4}^{(0)}\right)= \\
& =\mathcal{V}\left(X_{11}, X_{22}, X_{33}, X_{34}, X_{24}, X_{32}, X_{25}, X_{14}, X_{31}, 2 X_{12} X_{21}+2 X_{15} X_{51}+X_{16} X_{61},\right. \\
& \left.\left(X_{12} X_{21}+X_{15} X_{51}\right)^{2}+X_{16} X_{21}^{2} X_{52}-X_{15}^{2} X_{52} X_{61},-2 X_{15} X_{21} X_{52}\right)= \\
& =\mathcal{V}\left(X_{11}, X_{22}, X_{33}, X_{34}, X_{24}, X_{32}, X_{25}, X_{14}, X_{31}, X_{15}, 2 X_{12} X_{21}+X_{16} X_{61},\right. \\
& \left.\left(X_{12} X_{21}\right)^{2}+X_{16} X_{21}^{2} X_{52}\right) \cup \\
& \cup \mathcal{V}\left(X_{11}, X_{22}, X_{33}, X_{34}, X_{24}, X_{32}, X_{25}, X_{14}, X_{31}, X_{21}, 2 X_{15} X_{51}+X_{16} X_{61},\right. \\
& \left.\left(X_{15} X_{51}\right)^{2}-X_{15}^{2} X_{52} X_{61}\right) \cup \\
& \cup \mathcal{V}\left(X_{11}, X_{22}, X_{33}, X_{34}, X_{24}, X_{32}, X_{25}, X_{14}, X_{31}, X_{52}, 2 X_{12} X_{21}+2 X_{15} X_{51}+X_{16} X_{61},\right. \\
& \left.\left(X_{12} X_{21}+X_{15} X_{51}\right)^{2}\right)= \\
& =\mathcal{V}\left(X_{11}, X_{22}, X_{33}, X_{34}, X_{24}, X_{32}, X_{25}, X_{14}, X_{31}, X_{15}, X_{21}, X_{16} X_{61}\right) \cup \\
& \cup \mathcal{V}\left(X_{11}, X_{22}, X_{33}, X_{34}, X_{24}, X_{32}, X_{25}, X_{14}, X_{15}, X_{31}, 2 X_{12} X_{21}+X_{16} X_{61}, X_{12}^{2}+X_{16} X_{52}\right) \cup \\
& \cup \mathcal{V}\left(X_{11}, X_{22}, X_{33}, X_{34}, X_{24}, X_{32}, X_{25}, X_{14}, X_{31}, X_{21}, X_{15}, X_{16} X_{61}\right) \cup \\
& \cup \mathcal{V}\left(X_{11}, X_{22}, X_{33}, X_{34}, X_{24}, X_{32}, X_{25}, X_{14}, X_{31}, X_{21}, 2 X_{15} X_{51}+X_{16} X_{61}, X_{51}^{2}-X_{52} X_{61}\right) \cup \\
& \cup \mathcal{V}\left(X_{11}, X_{22}, X_{33}, X_{34}, X_{24}, X_{32}, X_{25}, X_{14}, X_{31}, X_{52}, X_{12} X_{21}+X_{15} X_{51}, X_{16} X_{61}\right)
\end{aligned}
$$

e as variedades abaixo são equidimensionais de dimensão $21-13=8$ :

$$
\begin{aligned}
& \mathcal{V}\left(X_{11}, X_{22}, X_{33}, X_{34}, X_{24}, X_{32}, X_{25}, X_{14}, X_{15}, X_{31}, 2 X_{12} X_{21}+X_{16} X_{61}, X_{12}^{2}+X_{16} X_{52}, X_{52}\right)= \\
& =\mathcal{V}\left(X_{11}, X_{22}, X_{33}, X_{34}, X_{24}, X_{32}, X_{25}, X_{14}, X_{15}, X_{31}, X_{52}, X_{12}, X_{16} X_{61}\right) \\
& \mathcal{V}\left(X_{11}, X_{22}, X_{33}, X_{34}, X_{24}, X_{32}, X_{25}, X_{14}, X_{31}, X_{21}, 2 X_{15} X_{51}+X_{16} X_{61}, X_{51}^{2}-X_{52} X_{61}, X_{52}\right)= \\
& =\mathcal{V}\left(X_{11}, X_{22}, X_{33}, X_{34}, X_{24}, X_{32}, X_{25}, X_{14}, X_{31}, X_{21}, X_{52}, X_{51}, X_{16} X_{61}\right) \\
& \mathcal{V}\left(X_{11}, X_{22}, X_{33}, X_{34}, X_{24}, X_{32}, X_{25}, X_{14}, X_{31}, X_{52}, X_{12} X_{21}+X_{15} X_{51}, X_{16} X_{61}, X_{15}\right)= \\
& =\mathcal{V}\left(X_{11}, X_{22}, X_{33}, X_{34}, X_{24}, X_{32}, X_{25}, X_{14}, X_{31}, X_{52}, X_{15}, X_{12} X_{21}, X_{16} X_{61}\right)
\end{aligned}
$$


Considere agora a variedade (4.8) acrescida de $X_{21}$. Ela é igual a:

$$
\begin{aligned}
& \mathcal{V}\left(X_{11}, X_{22}, X_{33}, X_{34}, X_{24}, X_{32}, X_{25}, X_{14}\right. \text {, } \\
& \left.-\left(X_{12} X_{23}+X_{15} X_{42}\right)^{2}+X_{52}\left(2 X_{13} X_{15} X_{23}-X_{16} X_{23}^{2}+X_{15}^{2} X_{43}\right), A, \phi_{4}^{(0)}, \partial_{X_{11}} \phi_{4}^{(0)}, X_{21}\right)= \\
& =\mathcal{V}\left(X_{11}, X_{22}, X_{33}, X_{34}, X_{24}, X_{32}, X_{25}, X_{14}, X_{21}\right. \text {, } \\
& -\left(X_{12} X_{23}+X_{15} X_{42}\right)^{2}+X_{52}\left(2 X_{13} X_{15} X_{23}-X_{16} X_{23}^{2}+X_{15}^{2} X_{43}\right) \text {, } \\
& 2 X_{13} X_{31}+2 X_{15} X_{51}+X_{16} X_{61} \text {, } \\
& \left(X_{13} X_{31}+X_{15} X_{51}\right)^{2}+X_{16} X_{31}^{2} X_{43}-2 X_{16} X_{23} X_{31} X_{51}-2 X_{15} X_{23} X_{31} X_{52}-X_{15}^{2} X_{52} X_{61} \text {, } \\
& \left.-2 X_{12} X_{23} X_{31}-2 X_{15} X_{31} X_{42}\right)= \\
& =\mathcal{V}\left(X_{11}, X_{22}, X_{33}, X_{34}, X_{24}, X_{32}, X_{25}, X_{14}, X_{21}, X_{31}\right. \text {, } \\
& -\left(X_{12} X_{23}+X_{15} X_{42}\right)^{2}+X_{52}\left(2 X_{13} X_{15} X_{23}-X_{16} X_{23}^{2}+X_{15}^{2} X_{43}\right), 2 X_{15} X_{51}+X_{16} X_{61} \text {, } \\
& \left.\left(X_{15} X_{51}\right)^{2}-X_{15}^{2} X_{52} X_{61}\right) \cup \\
& \cup \mathcal{V}\left(X_{11}, X_{22}, X_{33}, X_{34}, X_{24}, X_{32}, X_{25}, X_{14}, X_{21}, X_{12} X_{23}+X_{15} X_{42}\right. \text {, } \\
& X_{52}\left(2 X_{13} X_{15} X_{23}-X_{16} X_{23}^{2}+X_{15}^{2} X_{43}\right), 2 X_{13} X_{31}+2 X_{15} X_{51}+X_{16} X_{61} \text {, } \\
& \left.\left(X_{13} X_{31}+X_{15} X_{51}\right)^{2}+X_{16} X_{31}^{2} X_{43}-2 X_{16} X_{23} X_{31} X_{51}-2 X_{15} X_{23} X_{31} X_{52}-X_{15}^{2} X_{52} X_{61}\right)= \\
& =\mathcal{V}\left(X_{11}, X_{22}, X_{33}, X_{34}, X_{24}, X_{32}, X_{25}, X_{14}, X_{21}, X_{31}, X_{15}\right. \text {, } \\
& \left.-\left(X_{12} X_{23}\right)^{2}-X_{52} X_{16} X_{23}^{2}, X_{16} X_{61}\right) \cup \\
& \cup \mathcal{V}\left(X_{11}, X_{22}, X_{33}, X_{34}, X_{24}, X_{32}, X_{25}, X_{14}, X_{21}, X_{31}, X_{51}^{2}-X_{52} X_{61}\right. \text {, } \\
& -\left(X_{12} X_{23}+X_{15} X_{42}\right)^{2}+X_{52}\left(2 X_{13} X_{15} X_{23}-X_{16} X_{23}^{2}+X_{15}^{2} X_{43}\right) \text {, } \\
& \left.2 X_{15} X_{51}+X_{16} X_{61}\right) \cup \\
& \cup \mathcal{V}\left(X_{11}, X_{22}, X_{33}, X_{34}, X_{24}, X_{32}, X_{25}, X_{14}, X_{21}, X_{52}, X_{12} X_{23}+X_{15} X_{42}\right. \text {, } \\
& 2 X_{13} X_{31}+2 X_{15} X_{51}+X_{16} X_{61} \text {, } \\
& \left.\left(X_{13} X_{31}+X_{15} X_{51}\right)^{2}+X_{16} X_{31}^{2} X_{43}-2 X_{16} X_{23} X_{31} X_{51}\right) \cup \\
& \cup \mathcal{V}\left(X_{11}, X_{22}, X_{33}, X_{34}, X_{24}, X_{32}, X_{25}, X_{14}, X_{21}, X_{12} X_{23}+X_{15} X_{42}\right. \text {, } \\
& 2 X_{13} X_{15} X_{23}-X_{16} X_{23}^{2}+X_{15}^{2} X_{43}, 2 X_{13} X_{31}+2 X_{15} X_{51}+X_{16} X_{61} \text {, } \\
& \left.\left(X_{13} X_{31}+X_{15} X_{51}\right)^{2}+X_{16} X_{31}^{2} X_{43}-2 X_{16} X_{23} X_{31} X_{51}-2 X_{15} X_{23} X_{31} X_{52}-X_{15}^{2} X_{52} X_{61}\right)
\end{aligned}
$$

A variedade (4.9) é equidimensional de dimensão 8 pois a variedade

$$
\begin{aligned}
& \mathcal{V}\left(X_{11}, X_{22}, X_{33}, X_{34}, X_{24}, X_{32}, X_{25}, X_{14}, X_{21}, X_{31}, X_{15},-\left(X_{12} X_{23}\right)^{2}-X_{52} X_{16} X_{23}^{2}, X_{16} X_{61},\right. \\
& \left.X_{52}\right)=\mathcal{V}\left(X_{11}, X_{22}, X_{33}, X_{34}, X_{24}, X_{32}, X_{25}, X_{14}, X_{21}, X_{31}, X_{15}, X_{52}, X_{16} X_{61}, X_{12} X_{23}\right)
\end{aligned}
$$

é equidimensional de dimensão 21-14 $=7$.

A variedade (4.10) é equidimensional de dimensão 8 pois a variedade

$$
\begin{aligned}
& \mathcal{V}\left(X_{11}, X_{22}, X_{33}, X_{34}, X_{24}, X_{32}, X_{25}, X_{14}, X_{21}, X_{31}, X_{51}^{2}-X_{52} X_{61},\right. \\
& -\left(X_{12} X_{23}+X_{15} X_{42}\right)^{2}+X_{52}\left(2 X_{13} X_{15} X_{23}-X_{16} X_{23}^{2}+X_{15}^{2} X_{43}\right), 2 X_{15} X_{51}+X_{16} X_{61}, \\
& \left.X_{52}, X_{42}\right)= \\
& =\mathcal{V}\left(X_{11}, X_{22}, X_{33}, X_{34}, X_{24}, X_{32}, X_{25}, X_{14}, X_{21}, X_{31}, X_{52}, X_{42}, X_{51},-\left(X_{12} X_{23}\right)^{2},\right. \\
& \left.2 X_{15} X_{51}+X_{16} X_{61}\right)= \\
& =\mathcal{V}\left(X_{11}, X_{22}, X_{33}, X_{34}, X_{24}, X_{32}, X_{25}, X_{14}, X_{21}, X_{31}, X_{52}, X_{42}, X_{51}, X_{12} X_{23}, X_{16} X_{61}\right)
\end{aligned}
$$

é equidimensional de dimensão $21-15=6$. 
A variedade (4.11) é equidimensional de dimensão 8 pois a variedade

$$
\begin{aligned}
& \mathcal{V}\left(X_{11}, X_{22}, X_{33}, X_{34}, X_{24}, X_{32}, X_{25}, X_{14}, X_{21}, X_{52}, X_{12} X_{23}+X_{15} X_{42},\right. \\
& 2 X_{13} X_{31}+2 X_{15} X_{51}+X_{16} X_{61},\left(X_{13} X_{31}+X_{15} X_{51}\right)^{2}+X_{16} X_{31}^{2} X_{43}-2 X_{16} X_{23} X_{31} X_{51}, \\
& \left.X_{42}, X_{31}\right)=\mathcal{V}\left(X_{11}, X_{22}, X_{33}, X_{34}, X_{24}, X_{32}, X_{25}, X_{14}, X_{21}, X_{52}, X_{42}, X_{31}, X_{12} X_{23},\right. \\
& \left.2 X_{15} X_{51}+X_{16} X_{61}, X_{15} X_{51}\right)= \\
& =\mathcal{V}\left(X_{11}, X_{22}, X_{33}, X_{34}, X_{24}, X_{32}, X_{25}, X_{14}, X_{21}, X_{52}, X_{42}, X_{31}, X_{12} X_{23}, X_{15} X_{51}, X_{16} X_{61}\right)
\end{aligned}
$$

é equidimensional de dimensão $21-15=6$.

A variedade (4.12) acrescida de $X_{43}$ é igual a

$$
\begin{aligned}
& \mathcal{V}\left(X_{11}, X_{22}, X_{33}, X_{34}, X_{24}, X_{32}, X_{25}, X_{14}, X_{21}, X_{12} X_{23}+X_{15} X_{42}\right. \text {, } \\
& 2 X_{13} X_{15} X_{23}-X_{16} X_{23}^{2}+X_{15}^{2} X_{43}, 2 X_{13} X_{31}+2 X_{15} X_{51}+X_{16} X_{61} \text {, } \\
& \left(X_{13} X_{31}+X_{15} X_{51}\right)^{2}+X_{16} X_{31}^{2} X_{43}-2 X_{16} X_{23} X_{31} X_{51}-2 X_{15} X_{23} X_{31} X_{52}-X_{15}^{2} X_{52} X_{61} \text {, } \\
& \left.X_{43}\right)=\mathcal{V}\left(X_{11}, X_{22}, X_{33}, X_{34}, X_{24}, X_{32}, X_{25}, X_{14}, X_{21}, X_{43}, X_{12} X_{23}+X_{15} X_{42}\right. \text {, } \\
& 2 X_{13} X_{15} X_{23}-X_{16} X_{23}^{2}, 2 X_{13} X_{31}+2 X_{15} X_{51}+X_{16} X_{61} \text {, } \\
& \left.\left(X_{13} X_{31}+X_{15} X_{51}\right)^{2}-2 X_{16} X_{23} X_{31} X_{51}-2 X_{15} X_{23} X_{31} X_{52}-X_{15}^{2} X_{52} X_{61}\right)= \\
& =\mathcal{V}\left(X_{11}, X_{22}, X_{33}, X_{34}, X_{24}, X_{32}, X_{25}, X_{14}, X_{21}, X_{43}, X_{23}, X_{15} X_{42}\right. \text {, } \\
& \left.2 X_{13} X_{31}+2 X_{15} X_{51}+X_{16} X_{61},\left(X_{13} X_{31}+X_{15} X_{51}\right)^{2}-X_{15}^{2} X_{52} X_{61}\right) \cup \\
& \cup \mathcal{V}\left(X_{11}, X_{22}, X_{33}, X_{34}, X_{24}, X_{32}, X_{25}, X_{14}, X_{21}, X_{43}, X_{12} X_{23}+X_{15} X_{42}\right. \text {, } \\
& 2 X_{13} X_{15}-X_{16} X_{23}, 2 X_{13} X_{31}+2 X_{15} X_{51}+X_{16} X_{61} \text {, } \\
& \left.\left(X_{13} X_{31}+X_{15} X_{51}\right)^{2}-2 X_{16} X_{23} X_{31} X_{51}-2 X_{15} X_{23} X_{31} X_{52}-X_{15}^{2} X_{52} X_{61}\right)= \\
& =\mathcal{V}\left(X_{11}, X_{22}, X_{33}, X_{34}, X_{24}, X_{32}, X_{25}, X_{14}, X_{21}, X_{43}, X_{23}, X_{15}, X_{13} X_{31}, X_{16} X_{61}\right) \cup \\
& \cup \mathcal{V}\left(X_{11}, X_{22}, X_{33}, X_{34}, X_{24}, X_{32}, X_{25}, X_{14}, X_{21}, X_{43}, X_{23}, X_{42}\right. \text {, } \\
& \left.2 X_{13} X_{31}+2 X_{15} X_{51}+X_{16} X_{61},\left(X_{13} X_{31}+X_{15} X_{51}\right)^{2}-X_{15}^{2} X_{52} X_{61}\right) \cup \\
& \cup \mathcal{V}\left(X_{11}, X_{22}, X_{33}, X_{34}, X_{24}, X_{32}, X_{25}, X_{14}, X_{21}, X_{43}, X_{12} X_{23}+X_{15} X_{42}\right. \text {, } \\
& 2 X_{13} X_{15}-X_{16} X_{23}, 2 X_{13} X_{31}+2 X_{15} X_{51}+X_{16} X_{61} \text {, } \\
& \left.\left(X_{13} X_{31}+X_{15} X_{51}\right)^{2}-2 X_{16} X_{23} X_{31} X_{51}-2 X_{15} X_{23} X_{31} X_{52}-X_{15}^{2} X_{52} X_{61}\right)
\end{aligned}
$$

A variedade

$$
\begin{aligned}
& \mathcal{V}\left(X_{11}, X_{22}, X_{33}, X_{34}, X_{24}, X_{32}, X_{25}, X_{14}, X_{21}, X_{43}, X_{23}, X_{42},\right. \\
& \left.2 X_{13} X_{31}+2 X_{15} X_{51}+X_{16} X_{61},\left(X_{13} X_{31}+X_{15} X_{51}\right)^{2}-X_{15}^{2} X_{52} X_{61}, X_{15}\right)= \\
& =\mathcal{V}\left(X_{11}, X_{22}, X_{33}, X_{34}, X_{24}, X_{32}, X_{25}, X_{14}, X_{21}, X_{43}, X_{23}, X_{42}, X_{15}, X_{13} X_{31}, X_{16} X_{61}\right)
\end{aligned}
$$

é equidimensional de dimensão $21-15=6$. 
A variedade

$$
\begin{aligned}
& \mathcal{V}\left(X_{11}, X_{22}, X_{33}, X_{34}, X_{24}, X_{32}, X_{25}, X_{14}, X_{21}, X_{43}, X_{12} X_{23}+X_{15} X_{42}\right. \text {, } \\
& 2 X_{13} X_{15}-X_{16} X_{23}, 2 X_{13} X_{31}+2 X_{15} X_{51}+X_{16} X_{61} \text {, } \\
& \left.\left(X_{13} X_{31}+X_{15} X_{51}\right)^{2}-2 X_{16} X_{23} X_{31} X_{51}-2 X_{15} X_{23} X_{31} X_{52}-X_{15}^{2} X_{52} X_{61}, X_{42}\right)= \\
& =\mathcal{V}\left(X_{11}, X_{22}, X_{33}, X_{34}, X_{24}, X_{32}, X_{25}, X_{14}, X_{21}, X_{43}, X_{42}, X_{12} X_{23}\right. \text {, } \\
& 2 X_{13} X_{15}-X_{16} X_{23}, 2 X_{13} X_{31}+2 X_{15} X_{51}+X_{16} X_{61} \text {, } \\
& \left.\left(X_{13} X_{31}+X_{15} X_{51}\right)^{2}-2 X_{16} X_{23} X_{31} X_{51}-2 X_{15} X_{23} X_{31} X_{52}-X_{15}^{2} X_{52} X_{61}\right) \\
& =\mathcal{V}\left(X_{11}, X_{22}, X_{33}, X_{34}, X_{24}, X_{32}, X_{25}, X_{14}, X_{21}, X_{43}, X_{42}, X_{12}\right. \text {, } \\
& 2 X_{13} X_{15}-X_{16} X_{23}, 2 X_{13} X_{31}+2 X_{15} X_{51}+X_{16} X_{61} \text {, } \\
& \left.\left(X_{13} X_{31}+X_{15} X_{51}\right)^{2}-2 X_{16} X_{23} X_{31} X_{51}-2 X_{15} X_{23} X_{31} X_{52}-X_{15}^{2} X_{52} X_{61}\right) \cup \\
& \cup \mathcal{V}\left(X_{11}, X_{22}, X_{33}, X_{34}, X_{24}, X_{32}, X_{25}, X_{14}, X_{21}, X_{43}, X_{42}, X_{23}\right. \text {, } \\
& \left.2 X_{13} X_{15}, 2 X_{13} X_{31}+2 X_{15} X_{51}+X_{16} X_{61},\left(X_{13} X_{31}+X_{15} X_{51}\right)^{2}-X_{15}^{2} X_{52} X_{61}\right)= \\
& =\mathcal{V}\left(X_{11}, X_{22}, X_{33}, X_{34}, X_{24}, X_{32}, X_{25}, X_{14}, X_{21}, X_{43}, X_{42}, X_{12}\right. \text {, } \\
& 2 X_{13} X_{15}-X_{16} X_{23}, 2 X_{13} X_{31}+2 X_{15} X_{51}+X_{16} X_{61} \text {, } \\
& \left.\left(X_{13} X_{31}+X_{15} X_{51}\right)^{2}-2 X_{16} X_{23} X_{31} X_{51}-2 X_{15} X_{23} X_{31} X_{52}-X_{15}^{2} X_{52} X_{61}\right) \cup \\
& \cup \mathcal{V}\left(X_{11}, X_{22}, X_{33}, X_{34}, X_{24}, X_{32}, X_{25}, X_{14}, X_{21}, X_{43}, X_{42}, X_{23}, X_{13}, 2 X_{15} X_{51}+X_{16} X_{61}\right. \text {, } \\
& \left.\left(X_{15} X_{51}\right)^{2}-X_{15}^{2} X_{52} X_{61}\right) \cup \\
& \cup \mathcal{V}\left(X_{11}, X_{22}, X_{33}, X_{34}, X_{24}, X_{32}, X_{25}, X_{14}, X_{21}, X_{43}, X_{42}, X_{23}, X_{15}, X_{13} X_{31}, X_{16} X_{61}\right)
\end{aligned}
$$

é equidimensional de dimensão 6 pois a variedade

$\mathcal{V}\left(X_{11}, X_{22}, X_{33}, X_{34}, X_{24}, X_{32}, X_{25}, X_{14}, X_{21}, X_{43}, X_{42}, X_{12}\right.$,

$2 X_{13} X_{15}-X_{16} X_{23}, 2 X_{13} X_{31}+2 X_{15} X_{51}+X_{16} X_{61}$,

$\left.\left(X_{13} X_{31}+X_{15} X_{51}\right)^{2}-2 X_{16} X_{23} X_{31} X_{51}-2 X_{15} X_{23} X_{31} X_{52}-X_{15}^{2} X_{52} X_{61}, X_{23}, X_{52}\right)=$

$=\mathcal{V}\left(X_{11}, X_{22}, X_{33}, X_{34}, X_{24}, X_{32}, X_{25}, X_{14}, X_{21}, X_{43}, X_{42}, X_{12}, X_{23}, X_{52}\right.$,

$\left.2 X_{13} X_{15}, 2 X_{13} X_{31}+2 X_{15} X_{51}+X_{16} X_{61}, X_{13} X_{31}+X_{15} X_{51}\right)=$

$=\mathcal{V}\left(X_{11}, X_{22}, X_{33}, X_{34}, X_{24}, X_{32}, X_{25}, X_{14}, X_{21}, X_{43}, X_{42}, X_{12}, X_{23}, X_{52}, X_{13}, X_{15} X_{51}, X_{16} X_{61}\right) \cup$

$\cup \mathcal{V}\left(X_{11}, X_{22}, X_{33}, X_{34}, X_{24}, X_{32}, X_{25}, X_{14}, X_{21}, X_{43}, X_{42}, X_{12}, X_{23}, X_{52}, X_{15}, X_{13} X_{31}, X_{16} X_{61}\right)$

é equidimensional de dimensão $21-17=4$ e a variedade

$$
\begin{aligned}
& \mathcal{V}\left(X_{11}, X_{22}, X_{33}, X_{34}, X_{24}, X_{32}, X_{25}, X_{14}, X_{21}, X_{43}, X_{42}, X_{23}, X_{13}, 2 X_{15} X_{51}+X_{16} X_{61},\right. \\
& \left.\left(X_{15} X_{51}\right)^{2}-X_{15}^{2} X_{52} X_{61}, X_{52}\right)= \\
& =\mathcal{V}\left(X_{11}, X_{22}, X_{33}, X_{34}, X_{24}, X_{32}, X_{25}, X_{14}, X_{21}, X_{43}, X_{42}, X_{23}, X_{13}, X_{52}, X_{15} X_{51}, X_{16} X_{61}\right)
\end{aligned}
$$

é equidimensional de dimensão $21-16=5$.

Portanto, a variedade (4.12) acrescida de $X_{43}$ é equidimensional de dimensão 7 e, consequentemente, a variedade (4.12) é equidimensional de dimensão 8.

Assim concluimos que a variedade (4.8) acrescida de $X_{21}$ é equidimensional de dimensão 8 .

Logo, (4.8) é equidimensional de dimensão 9.

Por fim, $Z_{1}^{(1)}$ é equidimensional de dimensão 9.

(b) $Z_{2}^{(1)}$ e $Z_{4}^{(1)}$

$$
Z_{2}^{(1)}=\mathcal{V}\left(X_{11}, X_{22}, X_{33}, X_{34}, X_{24}, X_{32}, X_{25}, X_{23}, A, \phi_{6}^{(0)}, \phi_{4}^{(0)}, \partial_{F_{11}} \phi_{4}^{(0)}\right)
$$




$$
Z_{4}^{(1)}=\mathcal{V}\left(X_{11}, X_{22}, X_{33}, X_{34}, X_{24}, X_{32}, X_{25}, X_{52}, A, \phi_{6}^{(0)}, \phi_{4}^{(0)}, \partial_{F_{11}} \phi_{4}^{(0)}\right)
$$

Considere

$$
\begin{aligned}
Z_{2}^{(1)} \cap Z_{4}^{(1)} & =\mathcal{V}\left(X_{11}, X_{22}, X_{33}, X_{34}, X_{24}, X_{32}, X_{25}, X_{23}, X_{52}, A, \phi_{6}^{(0)}, \phi_{4}^{(0)}, \partial_{F_{11}} \phi_{4}^{(0)}\right)= \\
& =Z_{2}^{(1)}\left(X_{52}\right)=Z_{4}^{(1)}\left(X_{23}\right)
\end{aligned}
$$

Calculando o determinante da matriz $F$ do exemplo 4.3.3 para $X_{22}=X_{33}=X_{34}=X_{24}=X_{32}=X_{25}=X_{23}=X_{52}=0$, temos: (considerando $X_{11}=0$ )

$$
\begin{aligned}
\phi_{6}^{(0)} & =-X_{14}^{2} X_{21}^{2} X_{42}^{2}+2 X_{14} X_{15} X_{21} X_{31} X_{42}^{2}-X_{15}^{2} X_{31}^{2} X_{42}^{2}= \\
& =-\left(X_{42}^{2}\right)\left(X_{14} X_{21}-X_{15} X_{31}\right)^{2} \\
\phi_{4}^{(0)} & =X_{12}^{2} X_{21}^{2}+2 X_{12} X_{13} X_{21} X_{31}+X_{13}^{2} X_{31}^{2}+2 X_{12} X_{14} X_{21} X_{41}+ \\
& +2 X_{13} X_{14} X_{31} X_{41}+X_{14}^{2} X_{41}^{2}+2 X_{16} X_{21} X_{31} X_{42}+X_{16} X_{31}^{2} X_{43}+ \\
& +2 X_{12} X_{15} X_{21} X_{51}+2 X_{13} X_{15} X_{31} X_{51}+2 X_{14} X_{15} X_{41} X_{51}+X_{15}^{2} X_{51}^{2}- \\
& -2 X_{14} X_{15} X_{42} X_{61}-X_{14}^{2} X_{43} X_{61}= \\
& =\left(X_{12} X_{21}+X_{13} X_{31}+X_{14} X_{41}+X_{15} X_{51}\right)^{2}+2 X_{16} X_{21} X_{31} X_{42}+X_{16} X_{31}^{2} X_{43}- \\
& -2 X_{14} X_{15} X_{42} X_{61}-X_{14}^{2} X_{43} X_{61} \\
\partial_{X_{11}} \phi_{4}^{(0)} & =-2 X_{14} X_{21} X_{42}-2 X_{15} X_{31} X_{42}-2 X_{14} X_{31} X_{43}
\end{aligned}
$$

Além disso, $A=2 X_{12} X_{21}+2 X_{13} X_{31}+2 X_{14} X_{41}+2 X_{15} X_{51}+X_{16} X_{61}$.

Por $\phi_{6}^{(0)}$ concluimos que $Z_{2}^{(1)} \cap Z_{4}^{(1)}$ é a união das duas variedades abaixo.

$$
\mathcal{V}\left(X_{11}, X_{22}, X_{33}, X_{34}, X_{24}, X_{32}, X_{25}, X_{23}, X_{52}, X_{42}, A, \phi_{4}^{(0)}, \partial_{F_{11}} \phi_{4}^{(0)}\right)
$$

e

$$
\mathcal{V}\left(X_{11}, X_{22}, X_{33}, X_{34}, X_{24}, X_{32}, X_{25}, X_{23}, X_{52}, X_{14} X_{21}-X_{15} X_{31}, A, \phi_{4}^{(0)}, \partial_{F_{11}} \phi_{4}^{(0)}\right)
$$

A variedade 4.13 é equidimensional de dimensão 8 pois é igual a

$$
\begin{aligned}
& \mathcal{V}\left(X_{11}, X_{22}, X_{33}, X_{34}, X_{24}, X_{32}, X_{25}, X_{23}, X_{52}, X_{42}, A, \phi_{4}^{(0)}, \partial_{F_{11}} \phi_{4}^{(0)}\right)= \\
& =\mathcal{V}\left(X_{11}, X_{22}, X_{33}, X_{34}, X_{24}, X_{32}, X_{25}, X_{23}, X_{52}, X_{42}, A, \phi_{4}^{(0)},-2 X_{14} X_{31} X_{43}\right)= \\
& =\mathcal{V}\left(X_{11}, X_{22}, X_{33}, X_{34}, X_{24}, X_{32}, X_{25}, X_{23}, X_{52}, X_{42}, X_{14}, A,\right. \\
& \left.\left(X_{12} X_{21}+X_{13} X_{31}+X_{15} X_{51}\right)^{2}+X_{16} X_{31}^{2} X_{43}\right) \cup \\
& \cup \mathcal{V}\left(X_{11}, X_{22}, X_{33}, X_{34}, X_{24}, X_{32}, X_{25}, X_{23}, X_{52}, X_{42}, X_{31}, A,\right. \\
& \left.\left(X_{12} X_{21}+X_{14} X_{41}+X_{15} X_{51}\right)^{2}-X_{14}^{2} X_{43} X_{61}\right) \cup \\
& \cup \mathcal{V}\left(X_{11}, X_{22}, X_{33}, X_{34}, X_{24}, X_{32}, X_{25}, X_{23}, X_{52}, X_{42}, X_{43}, A,\right. \\
& \left.\left(X_{12} X_{21}+X_{13} X_{31}+X_{14} X_{41}+X_{15} X_{51}\right)^{2}\right)
\end{aligned}
$$


e as variedades

$$
\begin{aligned}
& \mathcal{V}\left(X_{11}, X_{22}, X_{33}, X_{34}, X_{24}, X_{32}, X_{25}, X_{23}, X_{52}, X_{42}, X_{14}, A,\right. \\
& \left.\left(X_{12} X_{21}+X_{13} X_{31}+X_{15} X_{51}\right)^{2}+X_{16} X_{31}^{2} X_{43}, X_{51}, X_{31}\right)= \\
& =\mathcal{V}\left(X_{11}, X_{22}, X_{33}, X_{34}, X_{24}, X_{32}, X_{25}, X_{23}, X_{52}, X_{42}, X_{14}, X_{51}, X_{31}, 2 X_{12} X_{21}+X_{16} X_{61},\right. \\
& \left.\left(X_{12} X_{21}\right)^{2}\right)= \\
& =\mathcal{V}\left(X_{11}, X_{22}, X_{33}, X_{34}, X_{24}, X_{32}, X_{25}, X_{23}, X_{52}, X_{42}, X_{14}, X_{51}, X_{31}, X_{12} X_{21}, X_{16} X_{61},\right)
\end{aligned}
$$

e

$$
\begin{aligned}
& \mathcal{V}\left(X_{11}, X_{22}, X_{33}, X_{34}, X_{24}, X_{32}, X_{25}, X_{23}, X_{52}, X_{42}, X_{31}, A,\right. \\
& \left.\left(X_{12} X_{21}+X_{14} X_{41}+X_{15} X_{51}\right)^{2}-X_{14}^{2} X_{43} X_{61}, X_{14}, X_{15}\right)= \\
& =\mathcal{V}\left(X_{11}, X_{22}, X_{33}, X_{34}, X_{24}, X_{32}, X_{25}, X_{23}, X_{52}, X_{42}, X_{31}, X_{14}, X_{15}, 2 X_{12} X_{21}+X_{16} X_{61},\right. \\
& \left.\left(X_{12} X_{21}\right)^{2}\right)= \\
& =\mathcal{V}\left(X_{11}, X_{22}, X_{33}, X_{34}, X_{24}, X_{32}, X_{25}, X_{23}, X_{52}, X_{42}, X_{31}, X_{14}, X_{15}, X_{12} X_{21}, X_{16} X_{61}\right)
\end{aligned}
$$

são equidimensionais de dimensão $21-15=6$ e a variedade

$$
\begin{aligned}
& \mathcal{V}\left(X_{11}, X_{22}, X_{33}, X_{34}, X_{24}, X_{32}, X_{25}, X_{23}, X_{52}, X_{42}, X_{43}, A,\right. \\
& \left.\left(X_{12} X_{21}+X_{13} X_{31}+X_{14} X_{41}+X_{15} X_{51}\right)^{2}, X_{31}, X_{41}, X_{51}\right)= \\
& =\mathcal{V}\left(X_{11}, X_{22}, X_{33}, X_{34}, X_{24}, X_{32}, X_{25}, X_{23}, X_{52}, X_{42}, X_{43}, X_{31}, X_{41}, X_{51},\right. \\
& \left.2 X_{12} X_{21}+X_{16} X_{61},\left(X_{12} X_{21}\right)^{2}\right)= \\
& =\mathcal{V}\left(X_{11}, X_{22}, X_{33}, X_{34}, X_{24}, X_{32}, X_{25}, X_{23}, X_{52}, X_{42}, X_{43}, X_{31}, X_{41}, X_{51}, X_{12} X_{21}, X_{16} X_{61}\right)
\end{aligned}
$$

é equidimensional de dimensão $21-16=5$. 
Considere agora a variedade (4.14) acrescida de $X_{42}$ :

$$
\begin{aligned}
& \mathcal{V}\left(X_{11}, X_{22}, X_{33}, X_{34}, X_{24}, X_{32}, X_{25}, X_{23}, X_{52}, X_{14} X_{21}-X_{15} X_{31}, A, \phi_{4}^{(0)}, \partial_{F_{11}} \phi_{4}^{(0)}, X_{42}\right)= \\
& =\mathcal{V}\left(X_{11}, X_{22}, X_{33}, X_{34}, X_{24}, X_{32}, X_{25}, X_{23}, X_{52}, X_{42}, X_{14} X_{21}-X_{15} X_{31}, A, \phi_{4}^{(0)}\right. \text {, } \\
& \left.-2 X_{14} X_{31} X_{43}\right)= \\
& =\mathcal{V}\left(X_{11}, X_{22}, X_{33}, X_{34}, X_{24}, X_{32}, X_{25}, X_{23}, X_{52}, X_{42}, X_{14}, X_{15} X_{31}\right. \text {, } \\
& 2 X_{12} X_{21}+2 X_{13} X_{31}+2 X_{15} X_{51}+X_{16} X_{61} \text {, } \\
& \left.\left(X_{12} X_{21}+X_{13} X_{31}+X_{15} X_{51}\right)^{2}+X_{16} X_{31}^{2} X_{43}\right) \cup \\
& \cup \mathcal{V}\left(X_{11}, X_{22}, X_{33}, X_{34}, X_{24}, X_{32}, X_{25}, X_{23}, X_{52}, X_{42}, X_{31}, X_{14} X_{21}\right. \text {, } \\
& 2 X_{12} X_{21}+2 X_{14} X_{41}+2 X_{15} X_{51}+X_{16} X_{61} \text {, } \\
& \left.\left(X_{12} X_{21}+X_{14} X_{41}+X_{15} X_{51}\right)^{2}-X_{14}^{2} X_{43} X_{61}\right) \cup \\
& \cup \mathcal{V}\left(X_{11}, X_{22}, X_{33}, X_{34}, X_{24}, X_{32}, X_{25}, X_{23}, X_{52}, X_{42}, X_{43}, X_{14} X_{21}-X_{15} X_{31}\right. \text {, } \\
& 2 X_{12} X_{21}+2 X_{13} X_{31}+2 X_{14} X_{41}+2 X_{15} X_{51}+X_{16} X_{61} \text {, } \\
& \left.X_{12} X_{21}+X_{13} X_{31}+X_{14} X_{41}+X_{15} X_{51}\right)= \\
& =\mathcal{V}\left(X_{11}, X_{22}, X_{33}, X_{34}, X_{24}, X_{32}, X_{25}, X_{23}, X_{52}, X_{42}, X_{14}, X_{15}\right. \text {, } \\
& \left.2 X_{12} X_{21}+2 X_{13} X_{31}+X_{16} X_{61},\left(X_{12} X_{21}+X_{13} X_{31}\right)^{2}+X_{16} X_{31}^{2} X_{43}\right) \cup \\
& \cup \mathcal{V}\left(X_{11}, X_{22}, X_{33}, X_{34}, X_{24}, X_{32}, X_{25}, X_{23}, X_{52}, X_{42}, X_{14}, X_{31}\right. \text {, } \\
& \left.2 X_{12} X_{21}+2 X_{15} X_{51}+X_{16} X_{61}, X_{12} X_{21}+X_{15} X_{51}\right) \cup \\
& \cup \mathcal{V}\left(X_{11}, X_{22}, X_{33}, X_{34}, X_{24}, X_{32}, X_{25}, X_{23}, X_{52}, X_{42}, X_{31}, X_{14}\right. \text {, } \\
& \left.2 X_{12} X_{21}+2 X_{15} X_{51}+X_{16} X_{61}, X_{12} X_{21}+X_{15} X_{51}\right) \cup \\
& \cup \mathcal{V}\left(X_{11}, X_{22}, X_{33}, X_{34}, X_{24}, X_{32}, X_{25}, X_{23}, X_{52}, X_{42}, X_{31}, X_{21}\right. \text {, } \\
& \left.2 X_{14} X_{41}+2 X_{15} X_{51}+X_{16} X_{61},\left(X_{14} X_{41}+X_{15} X_{51}\right)^{2}-X_{14}^{2} X_{43} X_{61}\right) \cup \\
& \cup \mathcal{V}\left(X_{11}, X_{22}, X_{33}, X_{34}, X_{24}, X_{32}, X_{25}, X_{23}, X_{52}, X_{42}, X_{43}, X_{14} X_{21}-X_{15} X_{31}\right. \text {, } \\
& \left.X_{12} X_{21}+X_{13} X_{31}+X_{14} X_{41}+X_{15} X_{51}, X_{16} X_{61}\right)
\end{aligned}
$$

A variedade (4.15) é equidimensional de dimensão 7 pois a variedade

$$
\begin{aligned}
& \mathcal{V}\left(X_{11}, X_{22}, X_{33}, X_{34}, X_{24}, X_{32}, X_{25}, X_{23}, X_{52}, X_{42}, X_{14}, X_{15},\right. \\
& \left.2 X_{12} X_{21}+2 X_{13} X_{31}+X_{16} X_{61},\left(X_{12} X_{21}+X_{13} X_{31}\right)^{2}+X_{16} X_{31}^{2} X_{43}, X_{31}\right)= \\
& =\mathcal{V}\left(X_{11}, X_{22}, X_{33}, X_{34}, X_{24}, X_{32}, X_{25}, X_{23}, X_{52}, X_{42}, X_{14}, X_{15}, X_{31}, X_{12} X_{21}, X_{16} X_{61}\right)
\end{aligned}
$$

é equidimensional de dimensão $21-15=6$.

As variedades (4.16) e (4.17) são iguais e são equidimensionais de dimensão 7 pois a variedade

$$
\begin{aligned}
& \mathcal{V}\left(X_{11}, X_{22}, X_{33}, X_{34}, X_{24}, X_{32}, X_{25}, X_{23}, X_{52}, X_{42}, X_{31}, X_{14}\right. \\
& \left.2 X_{12} X_{21}+2 X_{15} X_{51}+X_{16} X_{61}, X_{12} X_{21}+X_{15} X_{51}, X_{15}\right)= \\
& =\mathcal{V}\left(X_{11}, X_{22}, X_{33}, X_{34}, X_{24}, X_{32}, X_{25}, X_{23}, X_{52}, X_{42}, X_{31}, X_{14}, X_{15}, X_{12} X_{21}, X_{16} X_{61}\right)
\end{aligned}
$$

é equidimensional de dimensão $21-15=6$.

A variedade (4.18) é equidimensional de dimensão 7 pois a variedade

$$
\begin{aligned}
& \mathcal{V}\left(X_{11}, X_{22}, X_{33}, X_{34}, X_{24}, X_{32}, X_{25}, X_{23}, X_{52}, X_{42}, X_{31}, X_{21}\right. \\
& \left.2 X_{14} X_{41}+2 X_{15} X_{51}+X_{16} X_{61},\left(X_{14} X_{41}+X_{15} X_{51}\right)^{2}-X_{14}^{2} X_{43} X_{61}, X_{14}\right)= \\
& =\mathcal{V}\left(X_{11}, X_{22}, X_{33}, X_{34}, X_{24}, X_{32}, X_{25}, X_{23}, X_{52}, X_{42}, X_{31}, X_{21}, X_{14}, X_{15} X_{51}, X_{16} X_{61}\right)
\end{aligned}
$$

é equidimensional de dimensão $21-15=6$. 
A variedade (4.19) é equidimensional de dimensão 7 pois a variedade

$$
\begin{aligned}
& \mathcal{V}\left(X_{11}, X_{22}, X_{33}, X_{34}, X_{24}, X_{32}, X_{25}, X_{23}, X_{52}, X_{42}, X_{43}, X_{14} X_{21}-X_{15} X_{31},\right. \\
& \left.X_{12} X_{21}+X_{13} X_{31}+X_{14} X_{41}+X_{15} X_{51}, X_{16} X_{61}, X_{12}, X_{41}, X_{31}\right)= \\
& =\mathcal{V}\left(X_{11}, X_{22}, X_{33}, X_{34}, X_{24}, X_{32}, X_{25}, X_{23}, X_{52}, X_{42}, X_{43}, X_{12}, X_{41}, X_{31}, X_{14} X_{21}, X_{15} X_{51},\right. \\
& \left.X_{16} X_{61}\right)
\end{aligned}
$$

é equidimensional de dimensão $21-17=4$.

Portanto, a variedade (4.14) acrescida de $X_{42}$ é equidimensional de dimensão 7 e, consequentemente, a variedade (4.14) é equidimensional de dimensão 8.

Assim concluimos que $Z_{2}^{(1)} \cup Z_{4}^{(1)}$ é equidimensional de dimensão 8. Logo, $Z_{2}^{(1)}$ e $Z_{4}^{(1)}$ são equidimensionais de dimensão 9 .

(c) $Z_{3}^{(1)}$

$$
Z_{3}^{(1)}=\mathcal{V}\left(X_{11}, X_{22}, X_{33}, X_{34}, X_{24}, X_{32}, X_{25}, X_{31}, A, \phi_{6}^{(0)}, \phi_{4}^{(0)}, \partial_{X_{11}} \phi_{4}^{(0)}\right)
$$

Calculando o determinante da matriz $F$ do exemplo 4.3.3 para

$X_{22}=X_{33}=X_{34}=X_{24}=X_{32}=X_{25}=X_{31}=0$, temos:

(considerando $X_{11}=0$ )

$$
\begin{aligned}
\phi_{6}^{(0)} & =-X_{14}^{2} X_{21}^{2} X_{42}^{2}+2 X_{14}^{2} X_{21} X_{23} X_{42} X_{51}-X_{14}^{2} X_{23}^{2} X_{51}^{2}- \\
& -2 X_{14}^{2} X_{21} X_{23} X_{41} X_{52}+X_{14}^{2} X_{21}^{2} X_{43} X_{52}+X_{14}^{2} X_{23}^{2} X_{52} X_{61}= \\
& =X_{14}^{2}\left[-\left(X_{21} X_{42}-X_{23} X_{51}\right)^{2}+X_{52}\left(-2 X_{21} X_{23} X_{41}+X_{21}^{2} X_{43}+X_{23}^{2} X_{61}\right)\right] \\
\phi_{4}^{(0)} & =X_{12}^{2} X_{21}^{2}+2 X_{12} X_{14} X_{21} X_{41}+X_{14}^{2} X_{41}^{2}+2 X_{12} X_{15} X_{21} X_{51}+ \\
& +2 X_{14} X_{15} X_{41} X_{51}+X_{15}^{2} X_{51}^{2}+X_{16} X_{21}^{2} X_{52}+2 X_{14} X_{21} X_{23} X_{52}- \\
& -2 X_{12} X_{14} X_{23} X_{61}-2 X_{14} X_{15} X_{42} X_{61}-X_{14}^{2} X_{43} X_{61}-X_{15}^{2} X_{52} X_{61}= \\
& =\left(X_{12} X_{21}+X_{14} X_{41}+X_{15} X_{51}\right)^{2}+X_{16} X_{21}^{2} X_{52}+2 X_{14} X_{21} X_{23} X_{52}- \\
& -2 X_{12} X_{14} X_{23} X_{61}-2 X_{14} X_{15} X_{42} X_{61}-X_{14}^{2} X_{43} X_{61}-X_{15}^{2} X_{52} X_{61} \\
\partial_{X_{11}} \phi_{4}^{(0)} & =-2 X_{14} X_{21} X_{42}+2 X_{14} X_{23} X_{51}-2 X_{15} X_{21} X_{52}
\end{aligned}
$$

E $A=2 X_{12} X_{21}+2 X_{14} X_{41}+2 X_{15} X_{51}+X_{16} X_{61}$.

Seja $f$ o automorfismo do anel de polinômios nas 21 variáveis $X_{i j}$ definido da seguinte forma:

$$
\begin{aligned}
& f\left(X_{11}\right)=X_{11} \quad, \quad f\left(X_{12}\right)=X_{51} \quad, \quad f\left(X_{13}\right)=X_{41} \quad, \quad f\left(X_{14}\right)=-X_{31} \\
& f\left(X_{15}\right)=X_{21} \quad, \quad f\left(X_{16}\right)=-X_{61} \quad, \quad f\left(X_{21}\right)=X_{15} \quad, \quad f\left(X_{22}\right)=X_{22} \\
& f\left(X_{23}\right)=X_{23} \quad, \quad f\left(X_{24}\right)=-X_{24} \quad, \quad f\left(X_{25}\right)=X_{25} \quad, \quad f\left(X_{31}\right)=X_{14} \\
& f\left(X_{32}\right)=X_{32} \quad, \quad f\left(X_{33}\right)=X_{33} \quad, \quad f\left(X_{34}\right)=X_{34} \quad, \quad f\left(X_{41}\right)=-X_{13} \\
& f\left(X_{42}\right)=-X_{42} \quad, \quad f\left(X_{43}\right)=X_{43} \quad, \quad f\left(X_{51}\right)=X_{12} \quad, \quad f\left(X_{52}\right)=X_{52} \\
& f\left(X_{61}\right)=-X_{16}
\end{aligned}
$$

Assim, temos:

$$
\begin{aligned}
f\left(\phi_{6}^{(0)}\right) & =f\left(X_{14}^{2}\left[-\left(X_{21} X_{42}-X_{23} X_{51}\right)^{2}+X_{52}\left(-2 X_{21} X_{23} X_{41}+X_{21}^{2} X_{43}+X_{23}^{2} X_{61}\right)\right]\right)= \\
& =X_{31}^{2}\left[-\left(-X_{15} X_{42}-X_{23} X_{12}\right)^{2}+X_{52}\left(2 X_{15} X_{23} X_{13}+X_{15}^{2} X_{43}-X_{23}^{2} X_{16}\right)\right]
\end{aligned}
$$




$$
\begin{gathered}
f\left(\phi_{4}^{(0)}\right)=f\left(\left(X_{51} X_{15}+X_{31} X_{13}+X_{21} X_{12}\right)^{2}-X_{61} X_{15}^{2} X_{52}-2 X_{31} X_{15} X_{23} X_{52}-\right. \\
\left.-2 X_{51} X_{31} X_{23} X_{16}+2 X_{31} X_{21} X_{42} X_{16}+X_{31}^{2} X_{43} X_{16}+X_{21}^{2} X_{52} X_{16}\right) \\
f\left(\partial_{X_{11}}\right)=f\left(-2 X_{14} X_{21} X_{42}+2 X_{14} X_{23} X_{51}-2 X_{15} X_{21} X_{52}\right)= \\
=-2 X_{31} X_{15} X_{42}-2 X_{31} X_{23} X_{12}-2 X_{21} X_{15} X_{52} \\
f(A)=f\left(2 X_{12} X_{21}+2 X_{14} X_{41}+2 X_{15} X_{51}+X_{16} X_{61}\right)= \\
=2 X_{51} X_{15}+2 X_{31} X_{13}+2 X_{21} X_{12}+X_{61} X_{16}
\end{gathered}
$$

Dessa forma, temos:

$$
\begin{aligned}
f\left(Z_{3}^{(1)}\right):= & \mathcal{V}\left(f\left(X_{11}\right), f\left(X_{22}\right), f\left(X_{33}\right), f\left(X_{34}\right), f\left(X_{24}\right), f\left(X_{32}\right), f\left(X_{25}\right), f\left(X_{31}\right), f(A), f\left(\phi_{6}^{(0)}\right),\right. \\
& \left.f\left(\phi_{4}^{(0)}\right), f\left(\partial_{X_{11}} \phi_{4}^{(0)}\right)\right)=Z_{1}^{(1)}
\end{aligned}
$$

Portanto $Z_{3}^{(1)}$ e $Z_{1}^{(1)}$ são variedades isomórficas. Como $Z_{1}^{(1)}$ é equidimensional de dimensão 9 concluimos que $Z_{3}^{(1)}$ também é equidimensional de dimensão 9. Por fim, $W_{1}$ é equidimensional de dimensão 9 .

(ii) $W_{2}$

$$
\begin{aligned}
& W_{1}=\mathcal{V}\left(X_{11}, X_{22}, X_{33}, X_{34}, X_{24}, X_{32}, X_{25}, A, \phi_{6}^{(0)}, \partial_{X_{11}} \phi_{6}^{(0)}, \phi_{4}^{(0)}, \partial_{X_{11}} \phi_{4}^{(0)}\right) \\
& W_{2}=\mathcal{V}\left(X_{11}, X_{22}, X_{33}, X_{34}, X_{24}, X_{32}, X_{52}, A, \phi_{6}^{(0)}, \partial_{X_{11}} \phi_{6}^{(0)}, \phi_{4}^{(0)}, \partial_{X_{11}} \phi_{4}^{(0)}\right)
\end{aligned}
$$

Lembre-se que $\phi_{6}^{(0)}$ é o coeficiente independente e $\phi_{4}^{(0)}$ é o coeficiente de $u^{2}$ no polinômio obtido do determinante abaixo:

$$
\operatorname{det}\left(\begin{array}{cccccc}
u+X_{11} & X_{12} & X_{13} & X_{14} & X_{15} & X_{16} \\
X_{21} & u+X_{22} & X_{23} & X_{24} & X_{25} & X_{15} \\
X_{31} & X_{32} & u+X_{33} & X_{34} & X_{24} & X_{14} \\
X_{41} & X_{42} & X_{43} & u-X_{33} & -X_{23} & -X_{13} \\
X_{51} & X_{52} & X_{42} & -X_{32} & u-X_{22} & -X_{12} \\
X_{61} & X_{51} & X_{41} & -X_{31} & -X_{21} & u-X_{11}
\end{array}\right)
$$

Denote por $W_{1}\left(\phi_{6}^{(0)}\right)$ o elemento $\phi_{6}^{(0)}$ calculado considerando

$X_{22}=X_{33}=X_{34}=X_{24}=X_{32}=X_{25}$. De maneira análoga, defina $W_{1}(A), W_{1}\left(\partial_{X_{11}} \phi_{6}^{(0)}\right)$, $W_{1}\left(\phi_{4}^{(0)}\right)$ e $W_{1}\left(\partial_{X_{11}} \phi_{4}^{(0)}\right)$. Para obter esses elementos, devemos calcular o determinante abaixo:

$$
\operatorname{det}\left(\begin{array}{cccccc}
u+X_{11} & X_{12} & X_{13} & X_{14} & X_{15} & X_{16} \\
X_{21} & u & X_{23} & 0 & 0 & X_{15} \\
X_{31} & 0 & u & 0 & 0 & X_{14} \\
X_{41} & X_{42} & X_{43} & u & -X_{23} & -X_{13} \\
X_{51} & X_{52} & X_{42} & 0 & u & -X_{12} \\
X_{61} & X_{51} & X_{41} & -X_{31} & -X_{21} & u-X_{11}
\end{array}\right)
$$

Denote por $W_{2}\left(\phi_{6}^{(0)}\right)$ o elemento $\phi_{6}^{(0)}$ calculado considerando $X_{22}=X_{33}=X_{34}=X_{24}=X_{32}=X_{52}$. De maneira análoga, defina $W_{2}(A), W_{2}\left(\partial_{X_{11}} \phi_{6}^{(0)}\right)$, 
$W_{2}\left(\phi_{4}^{(0)}\right)$ e $W_{2}\left(\partial_{X_{11}} \phi_{4}^{(0)}\right)$. Para obter esses elementos, devemos calcular o determinante abaixo:

$$
\operatorname{det}\left(\begin{array}{cccccc}
u+X_{11} & X_{12} & X_{13} & X_{14} & X_{15} & X_{16} \\
X_{21} & u & X_{23} & 0 & X_{25} & X_{15} \\
X_{31} & 0 & u & 0 & 0 & X_{14} \\
X_{41} & X_{42} & X_{43} & u & -X_{23} & -X_{13} \\
X_{51} & 0 & X_{42} & 0 & u & -X_{12} \\
X_{61} & X_{51} & X_{41} & -X_{31} & -X_{21} & u-X_{11}
\end{array}\right)
$$

Nos dois casos não consideramos $X_{11}=0$ porque precisamos calcular as diferenciais em $X_{11}$. Considere agora o automorfismo $f$ do anel de polinômios nas 21 variáveis $X_{i j}$ definido da seguinte forma:

$$
\begin{aligned}
& f\left(X_{11}\right)=X_{11} \quad, \quad f\left(X_{12}\right)=X_{15} \quad, \quad f\left(X_{13}\right)=X_{13} \quad, \quad f\left(X_{16}\right)=-X_{16}, \quad f\left(X_{14}\right)=-X_{14} \\
& f\left(X_{15}\right)=X_{12} \quad, \quad f\left(X_{16}\right)=-X_{16} \quad, \quad f\left(X_{21}\right)=X_{51} \quad, \quad f\left(X_{22}\right)=X_{22} \\
& \left.f\left(X_{23}\right)=X_{42} \quad, \quad f\left(X_{24}\right)=X_{32} \quad, \quad f\left(X_{25}\right)=X_{52} \quad, \quad f\left(X_{33}\right)=X_{33}, \quad f\left(X_{34}\right)=-X_{34}\right)=X_{31} \\
& f\left(X_{32}\right)=X_{24} \quad, \quad f\left(X_{33}\right)=X_{33} \quad, \quad f\left(X_{34}\right)=-X_{34} \quad, \quad f\left(X_{41}\right)=-X_{41} \\
& f\left(X_{42}\right)=X_{23} \quad, \quad f\left(X_{43}\right)=-X_{43} \quad, \quad f\left(X_{51}\right)=X_{21} \quad, \quad f\left(X_{52}\right)=X_{25} \\
& f\left(X_{61}\right)=-X_{61}
\end{aligned}
$$

Para obter $f\left(W_{2}\left(\phi_{6}^{(0)}\right)\right), f\left(W_{2}\left(\partial_{X_{11}} \phi_{6}^{(0)}\right)\right), f\left(W_{2}\left(\phi_{4}^{(0)}\right)\right)$ e $f\left(W_{2}\left(\partial_{X_{11}} \phi_{4}^{(0)}\right)\right)$, basta calcular o determinante abaixo:

$$
\operatorname{det}\left(\begin{array}{cccccc}
u+X_{11} & X_{15} & X_{13} & -X_{14} & X_{12} & -X_{16} \\
X_{51} & u & X_{42} & 0 & X_{32} & X_{52} \\
X_{31} & 0 & u & 0 & 0 & -X_{14} \\
-X_{42} & X_{23} & -X_{43} & u & -X_{42} & -X_{13} \\
X_{21} & 0 & X_{23} & 0 & u & -X_{15} \\
-X_{61} & X_{51} & -X_{41} & -X_{31} & -X_{51} & u-X_{11}
\end{array}\right)
$$

que é o determinante (4.22) substituindo $X_{i j}$ por $f\left(X_{i j}\right)$.

Usando um programa, obtemos que (4.23) é igual a (4.62).

Assim podemos concluir que:

$$
\begin{array}{lll}
f\left(W_{2}\left(\phi_{6}^{(0)}\right)\right)=W_{1}\left(\phi_{6}^{(0)}\right), & & f\left(W_{2}\left(\partial_{X_{11}} \phi_{6}^{(0)}\right)\right)=W_{1}\left(\partial_{X_{11}} \phi_{6}^{(0)}\right) \\
f\left(W_{2}\left(\phi_{4}^{(0)}\right)\right)=W_{1}\left(\phi_{4}^{(0)}\right), & & f\left(W_{2}\left(\partial_{X_{11}} \phi_{4}^{(0)}\right)\right)=W_{1}\left(\partial_{X_{11}} \phi_{4}^{(0)}\right)
\end{array}
$$

e

$$
\begin{aligned}
& f\left(W_{2}\right):=\mathcal{V}\left(f\left(X_{11}\right), f\left(X_{22}\right), f\left(X_{33}\right), f\left(X_{34}\right), f\left(X_{24}\right), f\left(X_{32}\right), f\left(X_{52}\right), f(A), f\left(\phi_{6}^{(0)}\right),\right. \\
& \left.f\left(\partial_{X_{11}} \phi_{6}^{(0)}\right), f\left(\phi_{4}^{(0)}\right), f\left(\partial_{X_{11}} \phi_{4}^{(0)}\right)\right)= \\
& =\mathcal{V}\left(X_{11}, X_{22}, X_{33}, X_{34}, X_{24}, X_{32}, X_{25}, A, \phi_{6}^{(0)}, \partial_{X_{11}} \phi_{6}^{(0)}, \phi_{4}^{(0)}, \partial_{X_{11}} \phi_{4}^{(0)}\right)=W_{1}
\end{aligned}
$$

Assim concluimos que $W_{2}$ e $W_{1}$ são variedades isomórficas. Como $W_{1}$ é equidimensional de dimensão $9, W_{2}$ também é equidimensional de dimensão 9 .

(iii) $W_{4}$ 


$$
\begin{aligned}
& W_{3}=\mathcal{V}\left(X_{11}, X_{22}, X_{33}, X_{34}, X_{24}, 2 X_{23} X_{32}+X_{25} X_{52}, A, X_{23}^{2}+X_{25} X_{43}, \phi_{6}^{(0)}, \partial_{X_{11}} \phi_{6}^{(0)}, \phi_{4}^{(0)},\right. \\
& \left.\partial_{X_{11}} \phi_{4}^{(0)}\right) \\
& W_{4}=\mathcal{V}\left(X_{11}, X_{22}, X_{33}, X_{34}, X_{32}, 2 X_{24} X_{42}+X_{25} X_{52}, A, X_{42}^{2}-X_{43} X_{52}, \phi_{6}^{(0)}, \partial_{X_{11}} \phi_{6}^{(0)}, \phi_{4}^{(0)},\right. \\
& \left.\partial_{X_{11}} \phi_{4}^{(0)}\right)
\end{aligned}
$$

Denote por $W_{3}\left(\phi_{6}^{(0)}\right)$ o elemento $\phi_{6}^{(0)}$ calculado considerando $X_{22}=X_{33}=X_{34}=X_{24}=2 X_{23} X_{32}+X_{25} X_{52}=X_{23}^{2}+X_{25} X_{43}=0$. De maneira análoga, defina $W_{3}(A), W_{3}\left(\partial_{X_{11}} \phi_{6}^{(0)}\right), W_{3}\left(\phi_{4}^{(0)}\right)$ e $W_{3}\left(\partial_{X_{11}} \phi_{4}^{(0)}\right)$. Para obter esses elementos, devemos calcular o determinante abaixo:

$$
\operatorname{det}\left(\begin{array}{cccccc}
u+X_{11} & X_{12} & X_{13} & X_{14} & X_{15} & X_{16} \\
X_{21} & u & X_{23} & 0 & X_{25} & X_{15} \\
X_{31} & X_{32} & u & 0 & 0 & X_{14} \\
X_{41} & X_{42} & X_{43} & u & -X_{23} & -X_{13} \\
X_{51} & X_{52} & X_{42} & -X_{32} & u & -X_{12} \\
X_{61} & X_{51} & X_{41} & -X_{31} & -X_{21} & u-X_{11}
\end{array}\right)
$$

e considerar no resultado final $2 X_{23} X_{32}+X_{25} X_{52}=X_{23}^{2}+X_{25} X_{43}=0$.

Denote por $W_{4}\left(\phi_{6}^{(0)}\right)$ o elemento $\phi_{6}^{(0)}$ calculado considerando $X_{22}=X_{33}=X_{34}=X_{32}=2 X_{24} X_{42}+X_{25} X_{52}=X_{42}^{2}-X_{43} X_{52}=0$. De maneira análoga, defina $W_{4}(A), W_{4}\left(\partial_{X_{11}} \phi_{6}^{(0)}\right), W_{4}\left(\phi_{4}^{(0)}\right)$ e $W_{4}\left(\partial_{X_{11}} \phi_{4}^{(0)}\right)$. Para obter esses elementos, devemos calcular o determinante abaixo:

$$
\operatorname{det}\left(\begin{array}{cccccc}
u+X_{11} & X_{12} & X_{13} & X_{14} & X_{15} & X_{16} \\
X_{21} & u & X_{23} & X_{24} & X_{25} & X_{15} \\
X_{31} & 0 & u & 0 & X_{24} & X_{14} \\
X_{41} & X_{42} & X_{43} & u & -X_{23} & -X_{13} \\
X_{51} & X_{52} & X_{42} & 0 & u & -X_{12} \\
X_{61} & X_{51} & X_{41} & -X_{31} & -X_{21} & u-X_{11}
\end{array}\right)
$$

e considerar no resultado final $2 X_{24} X_{42}+X_{25} X_{52}=X_{42}^{2}-X_{43} X_{52}=0$.

Nos dois casos não consideramos $X_{11}=0$ porque precisamos calcular as diferenciais em $X_{11}$. Considere agora o automorfismo $f$ do anel de polinômios nas 21 variáveis $X_{i j}$ definido da seguinte forma:

$$
\begin{aligned}
& f\left(X_{11}\right)=X_{11} \quad, \quad f\left(X_{12}\right)=X_{21} \quad, \quad f\left(X_{13}\right)=X_{41} \quad, \quad f\left(X_{14}\right)=-X_{31} \\
& \left.f\left(X_{15}\right)=X_{51} \quad, \quad f\left(X_{16}\right)=X_{61} \quad, \quad f\left(X_{21}\right)=X_{12} \quad, \quad f\left(X_{24}\right)=-X_{32}\right)=X_{22} \\
& f\left(X_{23}\right)=X_{42} \quad, \quad f\left(X_{24}\right)=-X_{32} \quad, \quad f\left(X_{25}\right)=X_{52} \quad, \quad f\left(X_{33}\right)=X_{33}, f\left(X_{31}\right)=X_{14} \\
& f\left(X_{32}\right)=X_{24} \quad, \quad f\left(X_{33}\right)=X_{33} \quad, \quad f\left(X_{34}\right)=-X_{34} \quad, \quad f\left(X_{41}\right)=-X_{13} \\
& f\left(X_{42}\right)=-X_{23} \quad, \quad f\left(X_{43}\right)=-X_{43} \quad, \quad f\left(X_{51}\right)=X_{15} \quad, \quad f\left(X_{52}\right)=X_{25} \\
& f\left(X_{61}\right)=X_{16}
\end{aligned}
$$

Para obter $f\left(W_{4}\left(\phi_{6}^{(0)}\right)\right), f\left(W_{4}\left(\partial_{X_{11}} \phi_{6}^{(0)}\right)\right), f\left(W_{4}\left(\phi_{4}^{(0)}\right)\right)$ e $f\left(W_{4}\left(\partial_{X_{11}} \phi_{4}^{(0)}\right)\right)$, basta calcular o 
determinante abaixo:

$$
\operatorname{det}\left(\begin{array}{cccccc}
u+X_{11} & X_{21} & X_{41} & -X_{31} & X_{51} & X_{61} \\
X_{12} & u & X_{42} & -X_{32} & X_{52} & X_{51} \\
X_{14} & 0 & u & 0 & -X_{32} & -X_{31} \\
-X_{13} & -X_{23} & -X_{43} & u & -X_{42} & -X_{41} \\
X_{15} & X_{25} & -X_{23} & 0 & u & -X_{21} \\
X_{16} & X_{15} & -X_{13} & -X_{14} & -X_{12} & u-X_{11}
\end{array}\right)
$$

que é o determinante (4.25) substituindo $X_{i j}$ por $f\left(X_{i j}\right)$.

Usando um programa, obtemos que (4.26) é igual a (4.24).

Assim podemos concluir que:

$$
\begin{array}{lll}
f\left(W_{4}\left(\phi_{6}^{(0)}\right)\right)=W_{3}\left(\phi_{6}^{(0)}\right), & & f\left(W_{4}\left(\partial_{X_{11}} \phi_{6}^{(0)}\right)\right)=W_{3}\left(\partial_{X_{11}} \phi_{6}^{(0)}\right) \\
f\left(W_{4}\left(\phi_{4}^{(0)}\right)\right)=W_{3}\left(\phi_{4}^{(0)}\right), & & f\left(W_{4}\left(\partial_{X_{11}} \phi_{4}^{(0)}\right)\right)=W_{3}\left(\partial_{X_{11}} \phi_{4}^{(0)}\right)
\end{array}
$$

e

$$
\begin{aligned}
& f\left(W_{4}\right):=\mathcal{V}\left(f\left(X_{11}\right), f\left(X_{22}\right), f\left(X_{33}\right), f\left(X_{34}\right), f\left(X_{24}\right), f\left(X_{32}\right), f\left(X_{52}\right), f(A), f\left(\phi_{6}^{(0)}\right),\right. \\
& \left.f\left(\partial_{X_{11}} \phi_{6}^{(0)}\right), f\left(\phi_{4}^{(0)}\right), f\left(\partial_{X_{11}} \phi_{4}^{(0)}\right)\right)= \\
& =\mathcal{V}\left(X_{11}, X_{22}, X_{33}, X_{34}, X_{24}, X_{32}, X_{25}, A, \phi_{6}^{(0)}, \partial_{X_{11}} \phi_{6}^{(0)}, \phi_{4}^{(0)}, \partial_{X_{11}} \phi_{4}^{(0)}\right)=W_{3}
\end{aligned}
$$

Assim concluimos que $W_{4}$ e $W_{3}$ são variedades isomórficas. Então $W_{4}$ é equidimensional de dimensão 9 se e somente se $W_{3}$ é equidimensional de dimensão 9 .

(iv) $W_{5}$

$$
W_{5}=\mathcal{V}\left(X_{11}, X_{22}, X_{33}, X_{34}, X_{43}, X_{25}, X_{23} X_{32}+X_{24} X_{42}, A, \phi_{6}^{(0)}, \partial_{X_{11}} \phi_{6}^{(0)}, \phi_{4}^{(0)}, \partial_{X_{11}} \phi_{4}^{(0)}\right)
$$

Calculando o determinante da matriz $F$ do exemplo 4.3.3 para

$X_{22}=X_{33}=X_{34}=X_{43}=X_{25}=X_{23} X_{32}+X_{24} X_{42}=0$, temos (considerando $\left.X_{11}=0\right)$ :

$$
\begin{aligned}
\partial_{X_{11}} \phi_{6}^{(0)}= & 2 X_{14} X_{23}^{2} X_{31} X_{52}-2 X_{13} X_{23} X_{24} X_{31} X_{52}+2 X_{14} X_{23} X_{24} X_{41} X_{52}-2 X_{13} X_{24}^{2} X_{41} X_{52}= \\
& =2 X_{52}\left(X_{23} X_{31}+X_{24} X_{41}\right)\left(X_{14} X_{23}-X_{13} X_{24}\right)
\end{aligned}
$$

Assim, defina:

$$
\begin{aligned}
& Z_{1}^{(5)}=\mathcal{V}\left(X_{11}, X_{22}, X_{33}, X_{34}, X_{43}, X_{25}, X_{52}, X_{23} X_{32}+X_{24} X_{42}, A, \phi_{6}^{(0)}, \phi_{4}^{(0)}, \partial_{X_{11}} \phi_{4}^{(0)}\right) \\
& Z_{2}^{(5)}=\mathcal{V}\left(X_{11}, X_{22}, X_{33}, X_{34}, X_{43}, X_{25}, X_{23} X_{32}+X_{24} X_{42}, X_{23} X_{31}+X_{24} X_{41}, A, \phi_{6}^{(0)}, \phi_{4}^{(0)}, \partial_{X_{11}} \phi_{4}^{(0)}\right) \\
& Z_{3}^{(5)}=\mathcal{V}\left(X_{11}, X_{22}, X_{33}, X_{34}, X_{43}, X_{25}, X_{23} X_{32}+X_{24} X_{42}, X_{14} X_{23}-X_{13} X_{24}, A, \phi_{6}^{(0)}, \phi_{4}^{(0)}, \partial_{X_{11}} \phi_{4}^{(0)}\right)
\end{aligned}
$$

Temos $W_{5}=Z_{1}^{(5)} \cup Z_{2}^{(5)} \cup Z_{3}^{(5)}$.

(a) $Z_{1}^{(5)}$

Considere

$$
Z_{1}^{(5)}\left(X_{32}\right)=\mathcal{V}\left(X_{11}, X_{22}, X_{33}, X_{34}, X_{43}, X_{25}, X_{52}, X_{32}, X_{24} X_{42}, A, \phi_{6}^{(0)}, \phi_{4}^{(0)}, \partial_{X_{11}} \phi_{4}^{(0)}\right)
$$


Assim, $Z_{1}^{(5)}\left(X_{32}\right)$ é a união das duas variedades abaixo

$$
\mathcal{V}\left(X_{11}, X_{22}, X_{33}, X_{34}, X_{43}, X_{25}, X_{52}, X_{32}, X_{24}, A, \phi_{6}^{(0)}, \phi_{4}^{(0)}, \partial_{X_{11}} \phi_{4}^{(0)}\right)
$$

e

$$
\mathcal{V}\left(X_{11}, X_{22}, X_{33}, X_{34}, X_{43}, X_{25}, X_{52}, X_{32}, X_{42}, A, \phi_{6}^{(0)}, \phi_{4}^{(0)}, \partial_{X_{11}} \phi_{4}^{(0)}\right)
$$

Vamos provar que (4.27) é equidimensional de dimensão 8.

Calculando o determinante da matriz $F$ do exemplo 4.3.3 para

$X_{22}=X_{33}=X_{34}=X_{43}=X_{25}=X_{52}=X_{32}=X_{24}=0$, temos (considerando $\left.X_{11}=0\right)$ :

$$
\begin{aligned}
& \phi_{6}^{(0)}=-X_{12}^{2} X_{23}^{2} X_{31}^{2}+2 X_{12} X_{14} X_{21} X_{23} X_{31} X_{42}-2 X_{12} X_{15} X_{23} X_{31}^{2} X_{42}-X_{14}^{2} X_{21}^{2} X_{42}^{2}+ \\
&+2 X_{14} X_{15} X_{21} X_{31} X_{42}^{2}-X_{15}^{2} X_{31}^{2} X_{42}^{2}-2 X_{12} X_{14} X_{23}^{2} X_{31} X_{51}+2 X_{14}^{2} X_{21} X_{23} X_{42} X_{51}- \\
&-2 X_{14} X_{15} X_{23} X_{31} X_{42} X_{51}-X_{14}^{2} X_{23}^{2} X_{51}^{2}= \\
&=-\left(X_{12} X_{23} X_{31}-X_{14} X_{21} X_{42}+X_{15} X_{31} X_{42}+X_{14} X_{23} X_{51}\right)^{2} \\
& \phi_{4}^{(0)}= X_{12}^{2} X_{21}^{2}+2 X_{12} X_{13} X_{21} X_{31}+X_{13}^{2} X_{31}^{2}+2 X_{12} X_{14} X_{21} X_{41}+ \\
&+2 X_{13} X_{14} X_{31} X_{41}+X_{14}^{2} X_{41}^{2}+2 X_{16} X_{21} X_{31} X_{42}+2 X_{12} X_{15} X_{21} X_{51}+ \\
&+2 X_{13} X_{15} X_{31} X_{51}-2 X_{16} X_{23} X_{31} X_{51}+2 X_{14} X_{15} X_{41} X_{51}+X_{15}^{2} X_{51}^{2}- \\
&-2 X_{12} X_{14} X_{23} X_{61}-2 X_{14} X_{15} X_{42} X_{61}= \\
&=\left(X_{12} X_{21}+X_{13} X_{31}+X_{14} X_{41}+X_{15} X_{51}\right)^{2}+2 X_{16} X_{21} X_{31} X_{42}-2 X_{16} X_{23} X_{31} X_{51}- \\
&-2 X_{12} X_{14} X_{23} X_{61}-2 X_{14} X_{15} X_{42} X_{61} \\
& \partial_{X_{11}} \phi_{4}^{(0)}=-2 X_{12} X_{23} X_{31}-2 X_{14} X_{21} X_{42}-2 X_{15} X_{31} X_{42}+2 X_{14} X_{23} X_{51} \\
& \text { E } A=2 X_{12} X_{21}+2 X_{13} X_{31}+2 X_{14} X_{41}+2 X_{15} X_{51}+X_{16} X_{61} . \\
& \text { Por } \phi_{6}^{0} \text { e } \partial_{X_{11}} \phi_{4}^{(0)} \operatorname{concluimos} \text { que, para }(4.27), \text { temos } \\
& \quad X_{12} X_{23} X_{31}-X_{14} X_{21} X_{42}+X_{15} X_{31} X_{42}+X_{14} X_{23} X_{51}=0 \\
& \quad X_{12} X_{23} X_{31}+X_{14} X_{21} X_{42}+X_{15} X_{31} X_{42}-X_{14} X_{23} X_{51}=0
\end{aligned}
$$

e isso ocorre se e somente se:

$$
\begin{aligned}
& X_{14} X_{21} X_{42}-X_{14} X_{23} X_{51}=0 \\
& X_{12} X_{23} X_{31}+X_{15} X_{31} X_{42}=0
\end{aligned}
$$


Assim, (4.27) é igual a:

$$
\begin{aligned}
& \mathcal{V}\left(X_{11}, X_{22}, X_{33}, X_{34}, X_{43}, X_{25}, X_{32}, X_{24}, X_{52},\right. \\
& X_{14} X_{21} X_{42}-X_{14} X_{23} X_{51}, X_{12} X_{23} X_{31}+X_{15} X_{31} X_{42} \text {, } \\
& 2 X_{12} X_{21}+2 X_{13} X_{31}+2 X_{14} X_{41}+2 X_{15} X_{51}+X_{16} X_{61} \text {, } \\
& \left(X_{12} X_{21}+X_{13} X_{31}+X_{14} X_{41}+X_{15} X_{51}\right)^{2}+2 X_{16} X_{21} X_{31} X_{42}-2 X_{16} X_{23} X_{31} X_{51}- \\
& \left.-2 X_{12} X_{14} X_{23} X_{61}-2 X_{14} X_{15} X_{42} X_{61}\right)= \\
& =\mathcal{V}\left(X_{11}, X_{22}, X_{33}, X_{34}, X_{43}, X_{25}, X_{32}, X_{24}, X_{52}, X_{14}\right. \text {, } \\
& X_{12} X_{23} X_{31}+X_{15} X_{31} X_{42}, 2 X_{12} X_{21}+2 X_{13} X_{31}+2 X_{15} X_{51}+X_{16} X_{61} \text {, } \\
& \left.\left(X_{12} X_{21}+X_{13} X_{31}+X_{15} X_{51}\right)^{2}+2 X_{16} X_{21} X_{31} X_{42}-2 X_{16} X_{23} X_{31} X_{51}\right) \cup \\
& \cup \mathcal{V}\left(X_{11}, X_{22}, X_{33}, X_{34}, X_{43}, X_{25}, X_{32}, X_{24}, X_{52}, X_{21} X_{42}-X_{23} X_{51}\right. \text {, } \\
& X_{12} X_{23} X_{31}+X_{15} X_{31} X_{42}, 2 X_{12} X_{21}+2 X_{13} X_{31}+2 X_{14} X_{41}+2 X_{15} X_{51}+X_{16} X_{61} \text {, } \\
& \left(X_{12} X_{21}+X_{13} X_{31}+X_{14} X_{41}+X_{15} X_{51}\right)^{2}+2 X_{16} X_{21} X_{31} X_{42}-2 X_{16} X_{23} X_{31} X_{51}- \\
& \left.-2 X_{12} X_{14} X_{23} X_{61}-2 X_{14} X_{15} X_{42} X_{61}\right)= \\
& =\mathcal{V}\left(X_{11}, X_{22}, X_{33}, X_{34}, X_{43}, X_{25}, X_{32}, X_{24}, X_{52}, X_{14}, X_{31}\right. \text {, } \\
& \left.2 X_{12} X_{21}+2 X_{15} X_{51}+X_{16} X_{61}, X_{12} X_{21}+X_{15} X_{51}\right) \cup \\
& \cup \mathcal{V}\left(X_{11}, X_{22}, X_{33}, X_{34}, X_{43}, X_{25}, X_{32}, X_{24}, X_{52}, X_{14}, X_{12} X_{23}+X_{15} X_{42}\right. \text {, } \\
& 2 X_{12} X_{21}+2 X_{13} X_{31}+2 X_{15} X_{51}+X_{16} X_{61} \text {, } \\
& \left.\left(X_{12} X_{21}+X_{13} X_{31}+X_{15} X_{51}\right)^{2}+2 X_{16} X_{21} X_{31} X_{42}-2 X_{16} X_{23} X_{31} X_{51}\right) \cup \\
& \cup \mathcal{V}\left(X_{11}, X_{22}, X_{33}, X_{34}, X_{43}, X_{25}, X_{32}, X_{24}, X_{52}, X_{31}, X_{21} X_{42}-X_{23} X_{51}\right. \text {, } \\
& 2 X_{12} X_{21}+2 X_{14} X_{41}+2 X_{15} X_{51}+X_{16} X_{61} \text {, } \\
& \left.\left(X_{12} X_{21}+X_{14} X_{41}+X_{15} X_{51}\right)^{2}-2 X_{12} X_{14} X_{23} X_{61}-2 X_{14} X_{15} X_{42} X_{61}\right) \cup \\
& \cup \mathcal{V}\left(X_{11}, X_{22}, X_{33}, X_{34}, X_{43}, X_{25}, X_{32}, X_{24}, X_{52}, X_{21} X_{42}-X_{23} X_{51}, X_{12} X_{23}+X_{15} X_{42}\right. \text {, } \\
& 2 X_{12} X_{21}+2 X_{13} X_{31}+2 X_{14} X_{41}+2 X_{15} X_{51}+X_{16} X_{61} \text {, } \\
& \left(X_{12} X_{21}+X_{13} X_{31}+X_{14} X_{41}+X_{15} X_{51}\right)^{2}+2 X_{16} X_{21} X_{31} X_{42}-2 X_{16} X_{23} X_{31} X_{51}- \\
& \left.-2 X_{12} X_{14} X_{23} X_{61}-2 X_{14} X_{15} X_{42} X_{61}\right)
\end{aligned}
$$

A variedade (4.29) é equidimensional de dimensão 8 pois a variedade

$$
\begin{aligned}
& \mathcal{V}\left(X_{11}, X_{22}, X_{33}, X_{34}, X_{43}, X_{25}, X_{52}, X_{32}, X_{24}, X_{14}, X_{31},\right. \\
& \left.2 X_{12} X_{21}+2 X_{15} X_{51}+X_{16} X_{61}, X_{12} X_{21}+X_{15} X_{51}, X_{15}\right)= \\
& =\mathcal{V}\left(X_{11}, X_{22}, X_{33}, X_{34}, X_{43}, X_{25}, X_{52}, X_{32}, X_{24}, X_{14}, X_{31}, X_{15}, X_{12} X_{21}, X_{16} X_{61}\right)
\end{aligned}
$$

é equidimensional de dimensão $21-14=7$.

A variedade (4.30) é equidimensional de dimensão 8 pois a variedade

$$
\begin{aligned}
& \mathcal{V}\left(X_{11}, X_{22}, X_{33}, X_{34}, X_{43}, X_{25}, X_{52}, X_{32}, X_{24}, X_{14}, X_{12} X_{23}+X_{15} X_{42},\right. \\
& 2 X_{12} X_{21}+2 X_{13} X_{31}+2 X_{15} X_{51}+X_{16} X_{61}, \\
& \left.\left(X_{12} X_{21}+X_{13} X_{31}+X_{15} X_{51}\right)^{2}+2 X_{16} X_{21} X_{31} X_{42}-2 X_{16} X_{23} X_{31} X_{51}, X_{31}, X_{42}, X_{21}\right)= \\
& =\mathcal{V}\left(X_{11}, X_{22}, X_{33}, X_{34}, X_{43}, X_{25}, X_{52}, X_{32}, X_{24}, X_{14}, X_{31}, X_{42}, X_{21}, X_{12} X_{23}, X_{15} X_{51},\right. \\
& \left.X_{16} X_{61}\right)
\end{aligned}
$$

é equidimensional de dimensão $21-16=5$. 
A variedade (4.31) é equidimensional de dimensão 8 pois a variedade

$$
\begin{aligned}
& \mathcal{V}\left(X_{11}, X_{22}, X_{33}, X_{34}, X_{43}, X_{25}, X_{52}, X_{32}, X_{24}, X_{31}, X_{21} X_{42}-X_{23} X_{51},\right. \\
& 2 X_{12} X_{21}+2 X_{14} X_{41}+2 X_{15} X_{51}+X_{16} X_{61}, \\
& \left.\left(X_{12} X_{21}+X_{14} X_{41}+X_{15} X_{51}\right)^{2}-2 X_{12} X_{14} X_{23} X_{61}-2 X_{14} X_{15} X_{42} X_{61}, X_{14}, X_{23}, X_{12}\right)= \\
& =\mathcal{V}\left(X_{11}, X_{22}, X_{33}, X_{34}, X_{43}, X_{25}, X_{52}, X_{32}, X_{24}, X_{31}, X_{14}, X_{23}, X_{12}, X_{21} X_{42}, X_{15} X_{51},\right. \\
& \left.X_{16} X_{61}\right)
\end{aligned}
$$

é equidimensional de dimensão $21-16=5$.

A variedade (4.32) é equidimensional de dimensão 8 pois a variedade

$$
\begin{aligned}
& \mathcal{V}\left(X_{11}, X_{22}, X_{33}, X_{34}, X_{43}, X_{25}, X_{52}, X_{32}, X_{24}, X_{21} X_{42}-X_{23} X_{51}, X_{12} X_{23}+X_{15} X_{42},\right. \\
& 2 X_{12} X_{21}+2 X_{13} X_{31}+2 X_{14} X_{41}+2 X_{15} X_{51}+X_{16} X_{61}, \\
& \left(X_{12} X_{21}+X_{13} X_{31}+X_{14} X_{41}+X_{15} X_{51}\right)^{2}+2 X_{16} X_{21} X_{31} X_{42}-2 X_{16} X_{23} X_{31} X_{51}- \\
& \left.-2 X_{12} X_{14} X_{23} X_{61}-2 X_{14} X_{15} X_{42} X_{61}, X_{12}, X_{31}\right)= \\
& =\mathcal{V}\left(X_{11}, X_{22}, X_{33}, X_{34}, X_{43}, X_{25}, X_{52}, X_{32}, X_{24}, X_{12}, X_{31}, X_{21} X_{42}-X_{23} X_{51}, X_{15} X_{42},\right. \\
& \left.2 X_{14} X_{41}+2 X_{15} X_{51}+X_{16} X_{61},\left(X_{14} X_{41}+X_{15} X_{51}\right)^{2}-2 X_{14} X_{15} X_{42} X_{61}\right)= \\
& =\mathcal{V}\left(X_{11}, X_{22}, X_{33}, X_{34}, X_{43}, X_{25}, X_{52}, X_{32}, X_{24}, X_{12}, X_{31}, X_{15}, X_{21} X_{42}-X_{23} X_{51},\right. \\
& \left.X_{14} X_{41}, X_{16} X_{61}\right) \cup \\
& \cup \mathcal{V}\left(X_{11}, X_{22}, X_{33}, X_{34}, X_{43}, X_{25}, X_{52}, X_{32}, X_{24}, X_{12}, X_{31}, X_{42}, X_{23} X_{51}, X_{14} X_{41}+X_{15} X_{51},\right. \\
& \left.X_{16} X_{61}\right)= \\
& =\mathcal{V}\left(X_{11}, X_{22}, X_{33}, X_{34}, X_{43}, X_{25}, X_{52}, X_{32}, X_{24}, X_{12}, X_{31}, X_{15}, X_{21} X_{42}-X_{23} X_{51}, X_{14} X_{41},\right. \\
& \left.X_{16} X_{61}\right) \cup \\
& \cup \mathcal{V}\left(X_{11}, X_{22}, X_{33}, X_{34}, X_{43}, X_{25}, X_{52}, X_{32}, X_{24}, X_{12}, X_{31}, X_{42}, X_{23}, X_{14} X_{41}+X_{15} X_{51},\right. \\
& \left.X_{16} X_{61}\right) \cup \\
& \cup \mathcal{V}\left(X_{11}, X_{22}, X_{33}, X_{34}, X_{43}, X_{25}, X_{52}, X_{32}, X_{24}, X_{12}, X_{31}, X_{42}, X_{51}, X_{14} X_{41}, X_{16} X_{61}\right)
\end{aligned}
$$

é equidimensional de dimensão 6 já que as variedades abaixo são equidimensionais de dimensão $21-16=5$ :

$$
\begin{aligned}
& \mathcal{V}\left(X_{11}, X_{22}, X_{33}, X_{34}, X_{43}, X_{25}, X_{52}, X_{32}, X_{24}, X_{12}, X_{31}, X_{15}, X_{21} X_{42}-X_{23} X_{51}, X_{14} X_{41},\right. \\
& \left.X_{16} X_{61}, X_{23}\right)= \\
& =\mathcal{V}\left(X_{11}, X_{22}, X_{33}, X_{34}, X_{43}, X_{25}, X_{52}, X_{32}, X_{24}, X_{12}, X_{31}, X_{15}, X_{23}, X_{21} X_{42}, X_{14} X_{41}, X_{16} X_{61}\right)
\end{aligned}
$$

e

$\mathcal{V}\left(X_{11}, X_{22}, X_{33}, X_{34}, X_{43}, X_{25}, X_{52}, X_{32}, X_{24}, X_{12}, X_{31}, X_{42}, X_{23}, X_{14} X_{41}+X_{15} X_{51}\right.$, $\left.X_{16} X_{61}, X_{14}\right)=$

$=\mathcal{V}\left(X_{11}, X_{22}, X_{33}, X_{34}, X_{43}, X_{25}, X_{52}, X_{32}, X_{24}, X_{12}, X_{31}, X_{42}, X_{23}, X_{14}, X_{15} X_{51}, X_{16} X_{61}\right)$

Portanto, a variedade (4.27) é equidimensional de dimensão 8.

Agora vamos provar para (4.28).

Calculando o determinante da matriz $F$ do exemplo 4.3 .3 para 
$X_{22}=X_{33}=X_{34}=X_{43}=X_{25}=X_{52}=X_{32}=X_{42}=0$, temos (considerando $\left.X_{11}=0\right)$ :

$$
\begin{aligned}
\phi_{6}^{(0)}= & -X_{12}^{2} X_{23}^{2} X_{31}^{2}-2 X_{12}^{2} X_{23} X_{24} X_{31} X_{41}-X_{12}^{2} X_{24}^{2} X_{41}^{2}-2 X_{12} X_{14} X_{23}^{2} X_{31} X_{51}+ \\
& +2 X_{12} X_{13} X_{23} X_{24} X_{31} X_{51}-2 X_{12} X_{14} X_{23} X_{24} X_{41} X_{51}+2 X_{12} X_{13} X_{24}^{2} X_{41} X_{51}- \\
& -X_{14}^{2} X_{23}^{2} X_{51}^{2}+2 X_{13} X_{14} X_{23} X_{24} X_{51}^{2}-X_{13}^{2} X_{24}^{2} X_{51}^{2}= \\
& =-\left(X_{12} X_{23} X_{31}+X_{12} X_{24} X_{41}+X_{14} X_{23} X_{51}-X_{13} X_{24} X_{51}\right)^{2} \\
\phi_{4}^{(0)}= & X_{12}^{2} X_{21}^{2}+2 X_{12} X_{13} X_{21} X_{31}+X_{13}^{2} X_{31}^{2}+2 X_{12} X_{14} X_{21} X_{41}+ \\
& +2 X_{13} X_{14} X_{31} X_{41}+X_{14}^{2} X_{41}^{2}+2 X_{12} X_{15} X_{21} X_{51}+ \\
& +2 X_{13} X_{15} X_{31} X_{51}-2 X_{16} X_{23} X_{31} X_{51}+2 X_{14} X_{15} X_{41} X_{51}- \\
& -2 X_{16} X_{24} X_{41} X_{51}+X_{15}^{2} X_{51}^{2}-2 X_{12} X_{14} X_{23} X_{61}+2 X_{12} X_{13} X_{24} X_{61}= \\
& =\left(X_{12} X_{21}+X_{13} X_{31}+X_{14} X_{41}+X_{15} X_{51}\right)^{2}-2 X_{16} X_{23} X_{31} X_{51}- \\
& -2 X_{16} X_{24} X_{41} X_{51}-2 X_{12} X_{14} X_{23} X_{61}+2 X_{12} X_{13} X_{24} X_{61} \\
\partial_{X_{11}} \phi_{4}^{(0)}= & -2 X_{12} X_{23} X_{31}-2 X_{12} X_{24} X_{41}+2 X_{14} X_{23} X_{51}-2 X_{13} X_{24} X_{51}
\end{aligned}
$$

E $A=2 X_{12} X_{21}+2 X_{13} X_{31}+2 X_{14} X_{41}+2 X_{15} X_{51}+X_{16} X_{61}$.

Seja $f$ o automorfismo do anel de polinômios nas 21 variáveis $X_{i j}$ definido da seguinte forma:

$$
\begin{aligned}
& f\left(X_{11}\right)=X_{11} \quad, \quad f\left(X_{12}\right)=X_{14} \quad, \quad f\left(X_{13}\right)=X_{15} \quad, \quad f\left(X_{14}\right)=X_{12} \\
& f\left(X_{15}\right)=X_{13} \quad, \quad f\left(X_{16}\right)=-X_{16} \quad, \quad f\left(X_{21}\right)=X_{41} \quad, \quad f\left(X_{22}\right)=X_{22} \\
& f\left(X_{23}\right)=-X_{23} \quad, \quad f\left(X_{24}\right)=X_{42} \quad, \quad f\left(X_{25}\right)=X_{25} \quad, \quad f\left(X_{31}\right)=X_{51} \\
& f\left(X_{32}\right)=-X_{32} \quad, \quad f\left(X_{33}\right)=X_{33} \quad, \quad f\left(X_{34}\right)=X_{34} \quad, \quad f\left(X_{41}\right)=X_{21} \\
& f\left(X_{42}\right)=X_{24} \quad, \quad f\left(X_{43}\right)=X_{43} \quad, \quad f\left(X_{51}\right)=X_{31} \quad, \quad f\left(X_{52}\right)=X_{52} \\
& f\left(X_{61}\right)=-X_{61}
\end{aligned}
$$

Assim, temos:

$$
\begin{aligned}
f\left(\phi_{6}^{(0)}\right) & =f\left(-\left(X_{12} X_{23} X_{31}+X_{12} X_{24} X_{41}+X_{14} X_{23} X_{51}-X_{13} X_{24} X_{51}\right)^{2}\right)= \\
& =-\left(-X_{14} X_{23} X_{51}+X_{14} X_{42} X_{21}-X_{12} X_{23} X_{31}-X_{15} X_{42} X_{31}\right)^{2} \\
f\left(\phi_{4}^{(0)}\right)= & f\left(\left(X_{12} X_{21}+X_{13} X_{31}+X_{14} X_{41}+X_{15} X_{51}\right)^{2}-2 X_{16} X_{23} X_{31} X_{51}-\right. \\
& \left.-2 X_{16} X_{24} X_{41} X_{51}-2 X_{12} X_{14} X_{23} X_{61}+2 X_{12} X_{13} X_{24} X_{61}\right)= \\
= & \left(X_{14} X_{41}+X_{15} X_{51}+X_{12} X_{21}+X_{13} X_{31}\right)^{2}-2 X_{16} X_{23} X_{51} X_{31}+ \\
& +2 X_{16} X_{42} X_{21} X_{31}-2 X_{14} X_{12} X_{23} X_{61}-2 X_{14} X_{15} X_{42} X_{61} \\
f\left(\partial_{X_{11}} \phi_{4}^{(0)}\right) & =f\left(-2 X_{12} X_{23} X_{31}-2 X_{12} X_{24} X_{41}+2 X_{14} X_{23} X_{51}-2 X_{13} X_{24} X_{51}\right)= \\
& =2 X_{14} X_{23} X_{51}-2 X_{14} X_{42} X_{21}-2 X_{12} X_{23} X_{31}-2 X_{15} X_{42} X_{31} \\
f(A) & =f\left(2 X_{12} X_{21}+2 X_{13} X_{31}+2 X_{14} X_{41}+2 X_{15} X_{51}+X_{16} X_{61}\right)= \\
& =2 X_{14} X_{41}+2 X_{15} X_{51}+2 X_{12} X_{21}+2 X_{13} X_{31}+X_{16} X_{61}
\end{aligned}
$$

Dessa forma, temos:

$\mathcal{V}\left(f\left(X_{11}\right), f\left(X_{22}\right), f\left(X_{33}\right), f\left(X_{34}\right), f\left(X_{43}\right), f\left(X_{25}\right), f\left(X_{32}\right), f\left(X_{24}\right), f\left(X_{31}\right), f(A), f\left(\phi_{6}^{(0)}\right)\right.$, $\left.f\left(\phi_{4}^{(0)}\right), f\left(\partial_{X_{11}} \phi_{4}^{(0)}\right)\right)$ 
que é igual a variedade (4.27).

Como (4.27) é equidimensional de dimensão 8 concluimos que (4.28) também é equidimensional de dimensão 8.

Portanto $Z_{1}^{(5)}\left(X_{32}\right)$ é equidimensional de dimensão 8 e $Z_{1}^{(5)}$ é equidimensional de dimensão 9. (b) $Z_{2}^{(5)}$

$$
\begin{aligned}
Z_{2}^{(5)}= & \mathcal{V}\left(X_{11}, X_{22}, X_{33}, X_{34}, X_{43}, X_{25}, X_{23} X_{32}+X_{24} X_{42}, X_{23} X_{31}+X_{24} X_{41}, A, \phi_{6}^{(0)}, \phi_{4}^{(0)},\right. \\
& \left.\partial_{X_{11}} \phi_{4}^{(0)}\right)
\end{aligned}
$$

Considere a variedade:

$$
\begin{aligned}
Z_{2}^{(5)}\left(X_{42}\right)= & \mathcal{V}\left(X_{11}, X_{22}, X_{33}, X_{34}, X_{43}, X_{25}, X_{23} X_{32}+X_{24} X_{42}, X_{23} X_{31}+X_{24} X_{41}, A, \phi_{6}^{(0)}, \phi_{4}^{(0)},\right. \\
& \left.\partial_{X_{11}} \phi_{4}^{(0)}, X_{42}\right)
\end{aligned}
$$

e defina as seguintes variedades:

$$
\begin{aligned}
& H_{1}=\mathcal{V}\left(X_{11}, X_{22}, X_{33}, X_{34}, X_{43}, X_{25}, X_{42}, X_{23}, X_{24}, A, \phi_{6}^{(0)}, \phi_{4}^{(0)}, \partial_{X_{11}} \phi_{4}^{(0)}\right) \\
& H_{2}=\mathcal{V}\left(X_{11}, X_{22}, X_{33}, X_{34}, X_{43}, X_{25}, X_{42}, X_{23}, X_{41}, A, \phi_{6}^{(0)}, \phi_{4}^{(0)}, \partial_{X_{11}} \phi_{4}^{(0)}\right) \\
& H_{3}=\mathcal{V}\left(X_{11}, X_{22}, X_{33}, X_{34}, X_{43}, X_{25}, X_{42}, X_{32}, X_{23} X_{31}+X_{24} X_{41}, A, \phi_{6}^{(0)}, \phi_{4}^{(0)}, \partial_{X_{11}} \phi_{4}^{(0)}, X_{42}\right)
\end{aligned}
$$

Então temos $Z_{2}^{(5)}=H_{1} \cup H_{2} \cup H_{3}$. Assim, para provarmos que $Z_{2}^{(5)}$ é equidimensional de dimensão 9 basta provar que $H_{1}, H_{2}$ e $H_{3}$ são equidimensionais de dimensão 8 .

- $H_{1}$

Calculando o determinante da matriz $F$ do exemplo 4.3.3 para

$X_{22}=X_{33}=X_{34}=X_{43}=X_{25}=X_{42}=X_{23}=X_{24}=0$, temos (considerando $\left.X_{11}=0\right)$ :

$$
\begin{aligned}
& \phi_{6}^{(0)}=-X_{13}^{2} X_{21}^{2} X_{32}^{2}-2 X_{13} X_{15} X_{21} X_{32}^{2} X_{41}-X_{15}^{2} X_{32}^{2} X_{41}^{2}= \\
&=-X_{32}^{2}\left(X_{13} X_{21}+X_{15} X_{41}\right)^{2} \\
& \phi_{4}^{(0)}= X_{12}^{2} X_{21}^{2}+2 X_{12} X_{13} X_{21} X_{31}+X_{13}^{2} X_{31}^{2}+2 X_{12} X_{14} X_{21} X_{41}+ \\
&+2 X_{13} X_{14} X_{31} X_{41}-2 X_{16} X_{21} X_{32} X_{41}+X_{14}^{2} X_{41}^{2}+2 X_{12} X_{15} X_{21} X_{51}+ \\
&+2 X_{13} X_{15} X_{31} X_{51}+2 X_{14} X_{15} X_{41} X_{51}+X_{15}^{2} X_{51}^{2}+X_{16} X_{21}^{2} X_{52}- \\
&-2 X_{13} X_{15} X_{32} X_{61}-X_{15}^{2} X_{52} X_{61}= \\
&=\left(X_{12} X_{21}+X_{13} X_{31}+X_{14} X_{41}+X_{15} X_{51}\right)^{2}-2 X_{16} X_{21} X_{32} X_{41}+X_{16} X_{21}^{2} X_{52}- \\
&-2 X_{13} X_{15} X_{32} X_{61}-X_{15}^{2} X_{52} X_{61} \\
& \partial_{X_{11}} \phi_{4}^{(0)}=-2 X_{13} X_{21} X_{32}+2 X_{15} X_{32} X_{41}-2 X_{15} X_{21} X_{52} \\
& \text { E } A=2 X_{12} X_{21}+2 X_{13} X_{31}+2 X_{14} X_{41}+2 X_{15} X_{51}+X_{16} X_{61} . \\
& \text { Por } \phi_{6}^{(0)} \text { temos que } H_{1} \text { é a união das duas variedades abaixo: } \\
& \mathcal{V}\left(X_{11}, X_{22}, X_{33}, X_{34}, X_{43}, X_{25}, X_{42}, X_{23}, X_{24}, X_{32}, A, \phi_{4}^{(0)}, \partial_{X_{11}} \phi_{4}^{(0)}\right) \\
& \text { e } \quad \\
& \mathcal{V}\left(X_{11}, X_{22}, X_{33}, X_{34}, X_{43}, X_{25}, X_{42}, X_{23}, X_{24}, X_{13} X_{21}+X_{15} X_{41}, A, \phi_{4}^{(0)}, \partial_{X_{11}} \phi_{4}^{(0)}\right)
\end{aligned}
$$


A variedade (4.33) é igual a:

$$
\begin{aligned}
& \mathcal{V}\left(X_{11}, X_{22}, X_{33}, X_{34}, X_{43}, X_{25}, X_{42}, X_{23}, X_{24}, X_{32}, A, \phi_{4}^{(0)}, \partial_{X_{11}} \phi_{4}^{(0)}\right)= \\
& =\mathcal{V}\left(X_{11}, X_{22}, X_{33}, X_{34}, X_{43}, X_{25}, X_{42}, X_{23}, X_{24}, X_{32},\right. \\
& 2 X_{12} X_{21}+2 X_{13} X_{31}+2 X_{14} X_{41}+2 X_{15} X_{51}+X_{16} X_{61}, \\
& \left(X_{12} X_{21}+X_{13} X_{31}+X_{14} X_{41}+X_{15} X_{51}\right)^{2}+X_{16} X_{21}^{2} X_{52}-X_{15}^{2} X_{52} X_{61}, \\
& \left.-2 X_{15} X_{21} X_{52}\right)= \\
& =\mathcal{V}\left(X_{11}, X_{22}, X_{33}, X_{34}, X_{43}, X_{25}, X_{42}, X_{23}, X_{24}, X_{32}, X_{15},\right. \\
& \left.2 X_{12} X_{21}+2 X_{13} X_{31}+2 X_{14} X_{41}+X_{16} X_{61},\left(X_{12} X_{21}+X_{13} X_{31}+X_{14} X_{41}\right)^{2}+X_{16} X_{21}^{2} X_{52}\right) \cup \\
& \cup \mathcal{V}\left(X_{11}, X_{22}, X_{33}, X_{34}, X_{43}, X_{25}, X_{42}, X_{23}, X_{24}, X_{32}, X_{21},\right. \\
& \left.2 X_{13} X_{31}+2 X_{14} X_{41}+2 X_{15} X_{51}+X_{16} X_{61},\left(X_{13} X_{31}+X_{14} X_{41}+X_{15} X_{51}\right)^{2}-X_{15}^{2} X_{52} X_{61}\right) \cup \\
& \cup \mathcal{V}\left(X_{11}, X_{22}, X_{33}, X_{34}, X_{43}, X_{25}, X_{42}, X_{23}, X_{24}, X_{32}, X_{52},\right. \\
& \left.X_{12} X_{21}+X_{13} X_{31}+X_{14} X_{41}+X_{15} X_{51}, X_{16} X_{61}\right)
\end{aligned}
$$

As variedades

$$
\begin{aligned}
& \mathcal{V}\left(X_{11}, X_{22}, X_{33}, X_{34}, X_{43}, X_{25}, X_{42}, X_{23}, X_{24}, X_{32}, X_{15},\right. \\
& 2 X_{12} X_{21}+2 X_{13} X_{31}+2 X_{14} X_{41}+X_{16} X_{61},\left(X_{12} X_{21}+X_{13} X_{31}+X_{14} X_{41}\right)^{2}+X_{16} X_{21}^{2} X_{52}, \\
& \left.X_{21}, X_{31}\right)= \\
& =\mathcal{V}\left(X_{11}, X_{22}, X_{33}, X_{34}, X_{43}, X_{25}, X_{42}, X_{23}, X_{24}, X_{32}, X_{15}, X_{21}, X_{31}, X_{14} X_{41}, X_{16} X_{61}\right) \\
& \mathcal{V}\left(X_{11}, X_{22}, X_{33}, X_{34}, X_{43}, X_{25}, X_{42}, X_{23}, X_{24}, X_{32}, X_{21},\right. \\
& 2 X_{13} X_{31}+2 X_{14} X_{41}+2 X_{15} X_{51}+X_{16} X_{61},\left(X_{13} X_{31}+X_{14} X_{41}+X_{15} X_{51}\right)^{2}-X_{15}^{2} X_{52} X_{61}, \\
& \left.X_{15}, X_{13}\right)= \\
& =\mathcal{V}\left(X_{11}, X_{22}, X_{33}, X_{34}, X_{43}, X_{25}, X_{42}, X_{23}, X_{24}, X_{32}, X_{21}, X_{15}, X_{13}, X_{14} X_{41}, X_{16} X_{61}\right)
\end{aligned}
$$

são equidimensionais de dimensão $21-15=6$ e a variedade

$$
\begin{aligned}
& \mathcal{V}\left(X_{11}, X_{22}, X_{33}, X_{34}, X_{43}, X_{25}, X_{42}, X_{23}, X_{24}, X_{32}, X_{52},\right. \\
& \left.X_{12} X_{21}+X_{13} X_{31}+X_{14} X_{41}+X_{15} X_{51}, X_{16} X_{61}, X_{13}, X_{14}, X_{15}\right)= \\
& =\mathcal{V}\left(X_{11}, X_{22}, X_{33}, X_{34}, X_{43}, X_{25}, X_{42}, X_{23}, X_{24}, X_{32}, X_{52}, X_{13}, X_{14}, X_{15}, X_{12} X_{21}, X_{16} X_{61}\right)
\end{aligned}
$$

é equidimensional de dimensão $21-16=5$.

Então a variedade (4.33) é equidimensional de dimensão 8 . 
Considera a variedade (4.34) acrescida de $X_{52}$ :

$$
\begin{aligned}
& \mathcal{V}\left(X_{11}, X_{22}, X_{33}, X_{34}, X_{43}, X_{25}, X_{42}, X_{23}, X_{24}, X_{13} X_{21}+X_{15} X_{41}, A, \phi_{6}^{(0)}, \phi_{4}^{(0)}, \partial_{X_{11}} \phi_{4}^{(0)}, X_{52}\right)= \\
& =\mathcal{V}\left(X_{11}, X_{22}, X_{33}, X_{34}, X_{43}, X_{25}, X_{42}, X_{23}, X_{24}, X_{52}, X_{13} X_{21}+X_{15} X_{41}\right. \text {, } \\
& 2 X_{12} X_{21}+2 X_{13} X_{31}+2 X_{14} X_{41}+2 X_{15} X_{51}+X_{16} X_{61} \text {, } \\
& \left(X_{12} X_{21}+X_{13} X_{31}+X_{14} X_{41}+X_{15} X_{51}\right)^{2}-2 X_{16} X_{21} X_{32} X_{41}-2 X_{13} X_{15} X_{32} X_{61} \text {, } \\
& \left.-2 X_{13} X_{21} X_{32}+2 X_{15} X_{32} X_{41}\right)= \\
& =\mathcal{V}\left(X_{11}, X_{22}, X_{33}, X_{34}, X_{43}, X_{25}, X_{42}, X_{23}, X_{24}, X_{52}, X_{32}, X_{13} X_{21}+X_{15} X_{41}\right. \text {, } \\
& \left.X_{12} X_{21}+X_{13} X_{31}+X_{14} X_{41}+X_{15} X_{51}, X_{16} X_{61}\right) \cup \\
& \cup \mathcal{V}\left(X_{11}, X_{22}, X_{33}, X_{34}, X_{43}, X_{25}, X_{42}, X_{23}, X_{24}, X_{52}, X_{13} X_{21}+X_{15} X_{41},-X_{13} X_{21}+X_{15} X_{41}\right. \text {, } \\
& 2 X_{12} X_{21}+2 X_{13} X_{31}+2 X_{14} X_{41}+2 X_{15} X_{51}+X_{16} X_{61} \text {, } \\
& \left.\left(X_{12} X_{21}+X_{13} X_{31}+X_{14} X_{41}+X_{15} X_{51}\right)^{2}-2 X_{16} X_{21} X_{32} X_{41}-2 X_{13} X_{15} X_{32} X_{61}\right)= \\
& =\mathcal{V}\left(X_{11}, X_{22}, X_{33}, X_{34}, X_{43}, X_{25}, X_{42}, X_{23}, X_{24}, X_{52}, X_{32}, X_{13} X_{21}+X_{15} X_{41}\right. \text {, } \\
& \left.X_{12} X_{21}+X_{13} X_{31}+X_{14} X_{41}+X_{15} X_{51}, X_{16} X_{61}\right) \cup \\
& \cup \mathcal{V}\left(X_{11}, X_{22}, X_{33}, X_{34}, X_{43}, X_{25}, X_{42}, X_{23}, X_{24}, X_{52}, X_{13} X_{21}, X_{15} X_{41}\right. \text {, } \\
& 2 X_{12} X_{21}+2 X_{13} X_{31}+2 X_{14} X_{41}+2 X_{15} X_{51}+X_{16} X_{61} \text {, } \\
& \left.\left(X_{12} X_{21}+X_{13} X_{31}+X_{14} X_{41}+X_{15} X_{51}\right)^{2}-2 X_{16} X_{21} X_{32} X_{41}-2 X_{13} X_{15} X_{32} X_{61}\right)= \\
& =\mathcal{V}\left(X_{11}, X_{22}, X_{33}, X_{34}, X_{43}, X_{25}, X_{42}, X_{23}, X_{24}, X_{52}, X_{32}, X_{13} X_{21}+X_{15} X_{41}\right. \text {, } \\
& \left.X_{12} X_{21}+X_{13} X_{31}+X_{14} X_{41}+X_{15} X_{51}, X_{16} X_{61}\right) \cup \\
& \cup \mathcal{V}\left(X_{11}, X_{22}, X_{33}, X_{34}, X_{43}, X_{25}, X_{42}, X_{23}, X_{24}, X_{52}, X_{13}, X_{15}\right. \text {, } \\
& \left.2 X_{12} X_{21}+2 X_{14} X_{41}+X_{16} X_{61},\left(X_{12} X_{21}+X_{14} X_{41}\right)^{2}-2 X_{16} X_{21} X_{32} X_{41}\right) \cup \\
& \cup \mathcal{V}\left(X_{11}, X_{22}, X_{33}, X_{34}, X_{43}, X_{25}, X_{42}, X_{23}, X_{24}, X_{52}, X_{13}, X_{41}\right. \text {, } \\
& \left.X_{12} X_{21}+X_{15} X_{51}, X_{16} X_{61}\right) \cup \\
& \cup \mathcal{V}\left(X_{11}, X_{22}, X_{33}, X_{34}, X_{43}, X_{25}, X_{42}, X_{23}, X_{24}, X_{52}, X_{21}, X_{15}\right. \text {, } \\
& \left.X_{13} X_{31}+X_{14} X_{41}, X_{16} X_{61}\right) \cup \\
& \cup \mathcal{V}\left(X_{11}, X_{22}, X_{33}, X_{34}, X_{43}, X_{25}, X_{42}, X_{23}, X_{24}, X_{52}, X_{21}, X_{41}\right. \text {, } \\
& \left.\left.2 X_{13} X_{31}+2 X_{15} X_{51}+X_{16} X_{61},\left(X_{13} X_{31}+X_{15} X_{51}\right)^{2}-2 X_{13} X_{15} X_{32} X_{61}\right)\right)
\end{aligned}
$$

A variedade

$$
\begin{aligned}
& \mathcal{V}\left(X_{11}, X_{22}, X_{33}, X_{34}, X_{43}, X_{25}, X_{42}, X_{23}, X_{24}, X_{52}, X_{32}, X_{13} X_{21}+X_{15} X_{41},\right. \\
& \left.X_{12} X_{21}+X_{13} X_{31}+X_{14} X_{41}+X_{15} X_{51}, X_{16} X_{61}, X_{41}, X_{51}\right)= \\
& =\mathcal{V}\left(X_{11}, X_{22}, X_{33}, X_{34}, X_{43}, X_{25}, X_{42}, X_{23}, X_{24}, X_{52}, X_{32}, X_{41}, X_{51}, X_{13} X_{21},\right. \\
& \left.X_{12} X_{21}+X_{13} X_{31}, X_{16} X_{61}\right)= \\
& =\mathcal{V}\left(X_{11}, X_{22}, X_{33}, X_{34}, X_{43}, X_{25}, X_{42}, X_{23}, X_{24}, X_{52}, X_{32}, X_{41}, X_{51}, X_{13},\right. \\
& \left.X_{12} X_{21}, X_{16} X_{61}\right) \cup \\
& \cup \mathcal{V}\left(X_{11}, X_{22}, X_{33}, X_{34}, X_{43}, X_{25}, X_{42}, X_{23}, X_{24}, X_{52}, X_{32}, X_{41}, X_{51}, X_{21},\right. \\
& \left.X_{13} X_{31}, X_{16} X_{61}\right)
\end{aligned}
$$

é equidimensional de dimensão $21-16=5$ e as variedades abaixo são equidimensionais de dimensão $21-15=6$ :

$$
\begin{aligned}
& \mathcal{V}\left(X_{11}, X_{22}, X_{33}, X_{34}, X_{43}, X_{25}, X_{42}, X_{23}, X_{24}, X_{52}, X_{13}, X_{15}\right. \\
& \left.2 X_{12} X_{21}+2 X_{14} X_{41}+X_{16} X_{61},\left(X_{12} X_{21}+X_{14} X_{41}\right)^{2}-2 X_{16} X_{21} X_{32} X_{41}, X_{21}\right)= \\
& =\mathcal{V}\left(X_{11}, X_{22}, X_{33}, X_{34}, X_{43}, X_{25}, X_{42}, X_{23}, X_{24}, X_{52}, X_{13}, X_{15}, X_{21}, X_{14} X_{41}, X_{16} X_{61}\right)
\end{aligned}
$$




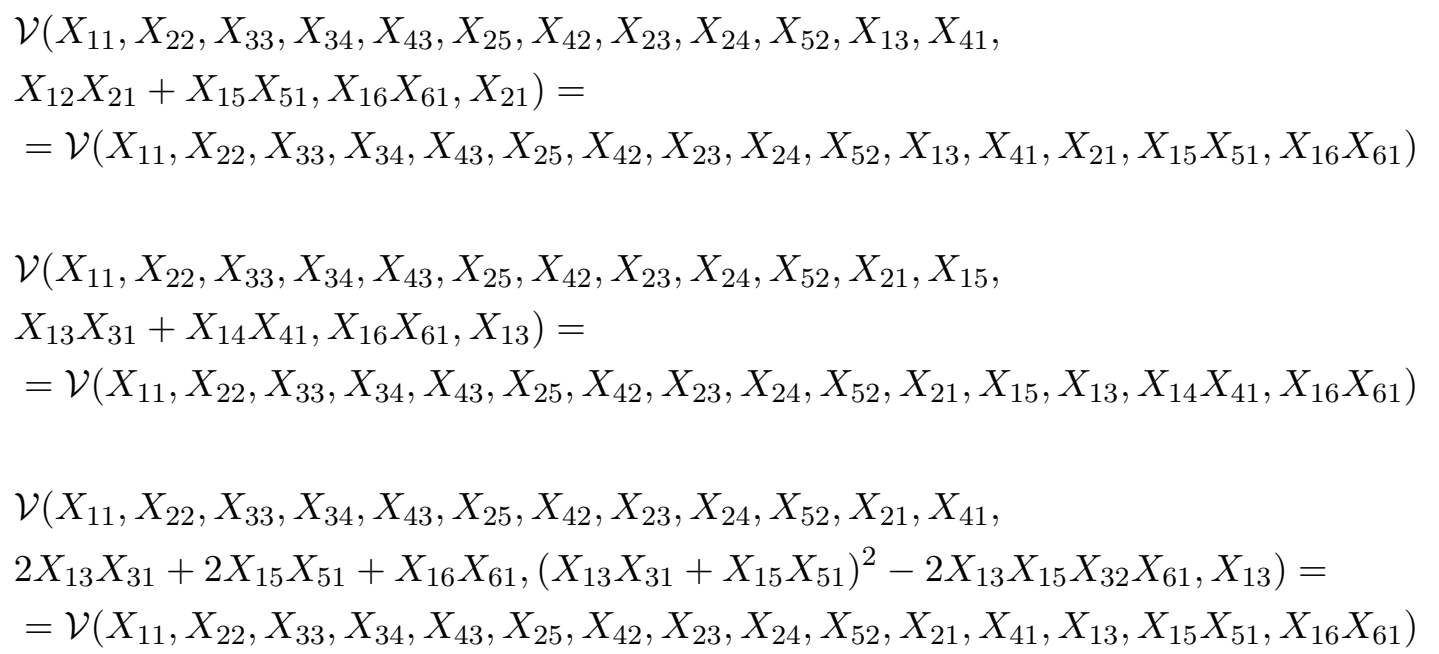

Então a variedade (4.34) acrescida de $X_{52}$ é equidimensional de dimensão 7 e a variedade (4.34) é equidimensional de dimensão 8.

Portanto a variedade $H_{1}$ é equidimensional de dimensão 8.

- $\mathrm{H}_{2}$

$$
H_{2}=\mathcal{V}\left(X_{11}, X_{22}, X_{33}, X_{34}, X_{43}, X_{25}, X_{42}, X_{23}, X_{41}, A, \phi_{6}^{(0)}, \phi_{4}^{(0)}, \partial_{X_{11}} \phi_{4}^{(0)}\right)
$$

Calculando o determinante da matriz $F$ do exemplo 4.3.3 para $X_{22}=X_{33}=X_{34}=X_{43}=X_{25}=X_{42}=X_{23}=X_{41}=0$, temos (considerando $X_{11}=0$ ):

$$
\begin{aligned}
\phi_{6}^{(0)}= & -X_{13}^{2} X_{21}^{2} X_{32}^{2}-2 X_{13}^{2} X_{21} X_{24} X_{32} X_{51}-X_{13}^{2} X_{24}^{2} X_{51}^{2}+2 X_{13}^{2} X_{21} X_{24} X_{31} X_{52}+ \\
& +X_{13}^{2} X_{24}^{2} X_{52} X_{61}=X_{13}^{2}\left[-\left(X_{21} X_{32}+X_{24} X_{51}\right)^{2}+X_{24} X_{52}\left(2 X_{21} X_{31}+X_{24} X_{61}\right)\right] \\
\phi_{4}^{(0)}= & X_{12}^{2} X_{21}^{2}+2 X_{12} X_{13} X_{21} X_{31}+X_{13}^{2} X_{31}^{2}+2 X_{12} X_{15} X_{21} X_{51}+ \\
& +2 X_{13} X_{15} X_{31} X_{51}+X_{15}^{2} X_{51}^{2}+X_{16} X_{21}^{2} X_{52}-2 X_{13} X_{21} X_{24} X_{52}+ \\
& +2 X_{12} X_{13} X_{24} X_{61}-2 X_{13} X_{15} X_{32} X_{61}-X_{15}^{2} X_{52} X_{61}= \\
= & \left(X_{12} X_{21}+X_{13} X_{31}+X_{15} X_{51}\right)^{2}+X_{16} X_{21}^{2} X_{52}-2 X_{13} X_{21} X_{24} X_{52}+ \\
& +2 X_{12} X_{13} X_{24} X_{61}-2 X_{13} X_{15} X_{32} X_{61}-X_{15}^{2} X_{52} X_{61} \\
\partial_{X_{11}} \phi_{4}^{(0)}= & -2 X_{13} X_{21} X_{32}-2 X_{13} X_{24} X_{51}-2 X_{15} X_{21} X_{52}
\end{aligned}
$$

E $A=2 X_{12} X_{21}+2 X_{13} X_{31}+2 X_{15} X_{51}+X_{16} X_{61}$.

Por $\phi_{6}^{(0)}$ temos que $H_{2}$ é a união das duas variedades abaixo:

$$
\mathcal{V}\left(X_{11}, X_{22}, X_{33}, X_{34}, X_{43}, X_{25}, X_{42}, X_{23}, X_{24}, X_{13}, A, \phi_{4}^{(0)}, \partial_{X_{11}} \phi_{4}^{(0)}\right)
$$

e

$$
\begin{aligned}
& \mathcal{V}\left(X_{11}, X_{22}, X_{33}, X_{34}, X_{43}, X_{25}, X_{42}, X_{23}, X_{24},\right. \\
& \left.-\left(X_{21} X_{32}+X_{24} X_{51}\right)^{2}+X_{24} X_{52}\left(2 X_{21} X_{31}+X_{24} X_{61}\right), A, \phi_{4}^{(0)}, \partial_{X_{11}} \phi_{4}^{(0)}\right)
\end{aligned}
$$


A variedade (4.35) é igual a:

$$
\begin{aligned}
& \mathcal{V}\left(X_{11}, X_{22}, X_{33}, X_{34}, X_{43}, X_{25}, X_{42}, X_{23}, X_{24}, X_{13}, A, \phi_{4}^{(0)}, \partial_{X_{11}} \phi_{4}^{(0)}\right)= \\
& =\mathcal{V}\left(X_{11}, X_{22}, X_{33}, X_{34}, X_{43}, X_{25}, X_{42}, X_{23}, X_{24}, X_{13}, 2 X_{12} X_{21}+2 X_{15} X_{51}+X_{16} X_{61},\right. \\
& \left.\left(X_{12} X_{21}+X_{15} X_{51}\right)^{2}+X_{16} X_{21}^{2} X_{52}-X_{15}^{2} X_{52} X_{61},-2 X_{15} X_{21} X_{52}\right)= \\
& =\mathcal{V}\left(X_{11}, X_{22}, X_{33}, X_{34}, X_{43}, X_{25}, X_{42}, X_{23}, X_{24}, X_{13}, X_{15}, 2 X_{12} X_{21}+X_{16} X_{61},\right. \\
& \left.\left(X_{12} X_{21}\right)^{2}+X_{16} X_{21}^{2} X_{52}\right) \cup \\
& \cup \mathcal{V}\left(X_{11}, X_{22}, X_{33}, X_{34}, X_{43}, X_{25}, X_{42}, X_{23}, X_{24}, X_{13}, X_{21}, 2 X_{15} X_{51}+X_{16} X_{61},\right. \\
& \left.\left(X_{15} X_{51}\right)^{2}-X_{15}^{2} X_{52} X_{61}\right) \cup \\
& \cup \mathcal{V}\left(X_{11}, X_{22}, X_{33}, X_{34}, X_{43}, X_{25}, X_{42}, X_{23}, X_{24}, X_{13}, X_{52}, X_{12} X_{21}+X_{15} X_{51}, X_{16} X_{61}\right)
\end{aligned}
$$

As variedades abaixo são equidimensionais de dimensão 21-14=7:

$$
\begin{aligned}
& \mathcal{V}\left(X_{11}, X_{22}, X_{33}, X_{34}, X_{43}, X_{25}, X_{42}, X_{23}, X_{24}, X_{13}, X_{15}, 2 X_{12} X_{21}+X_{16} X_{61},\right. \\
& \left.\left(X_{12} X_{21}\right)^{2}+X_{16} X_{21}^{2} X_{52}, X_{52}\right)= \\
& =\mathcal{V}\left(X_{11}, X_{22}, X_{33}, X_{34}, X_{43}, X_{25}, X_{42}, X_{23}, X_{24}, X_{13}, X_{15}, X_{52}, X_{12} X_{21}, X_{16} X_{61}\right) \\
& \mathcal{V}\left(X_{11}, X_{22}, X_{33}, X_{34}, X_{43}, X_{25}, X_{42}, X_{23}, X_{24}, X_{13}, X_{21}, 2 X_{15} X_{51}+X_{16} X_{61},\right. \\
& \left.\left(X_{15} X_{51}\right)^{2}-X_{15}^{2} X_{52} X_{61}, X_{52}\right)= \\
& =\mathcal{V}\left(X_{11}, X_{22}, X_{33}, X_{34}, X_{43}, X_{25}, X_{42}, X_{23}, X_{24}, X_{13}, X_{21}, X_{52}, X_{15} X_{51}, X_{16} X_{61}\right)
\end{aligned}
$$

$\mathcal{V}\left(X_{11}, X_{22}, X_{33}, X_{34}, X_{43}, X_{25}, X_{42}, X_{23}, X_{24}, X_{13}, X_{52}, X_{12} X_{21}+X_{15} X_{51}, X_{16} X_{61}, X_{15}\right)=$ $=\mathcal{V}\left(X_{11}, X_{22}, X_{33}, X_{34}, X_{43}, X_{25}, X_{42}, X_{23}, X_{24}, X_{13}, X_{52}, X_{15}, X_{12} X_{21}, X_{16} X_{61}\right)$

Então a variedade (4.35) é equidimensional de dimensão 8. 
Considere agora a variedade (4.36) acrescida de $X_{15}$ :

$$
\begin{aligned}
& \mathcal{V}\left(X_{11}, X_{22}, X_{33}, X_{34}, X_{43}, X_{25}, X_{42}, X_{23}, X_{41}\right. \text {, } \\
& \left.-\left(X_{21} X_{32}+X_{24} X_{51}\right)^{2}+X_{24} X_{52}\left(2 X_{21} X_{31}+X_{24} X_{61}\right), A, \phi_{4}^{(0)}, \partial_{X_{11}} \phi_{4}^{(0)}, X_{15}\right)= \\
& =\mathcal{V}\left(X_{11}, X_{22}, X_{33}, X_{34}, X_{43}, X_{25}, X_{42}, X_{23}, X_{41}, X_{15}\right. \text {, } \\
& -\left(X_{21} X_{32}+X_{24} X_{51}\right)^{2}+X_{24} X_{52}\left(2 X_{21} X_{31}+X_{24} X_{61}\right), 2 X_{12} X_{21}+2 X_{13} X_{31}+X_{16} X_{61} \text {, } \\
& \left(X_{12} X_{21}+X_{13} X_{31}\right)^{2}+X_{16} X_{21}^{2} X_{52}-2 X_{13} X_{21} X_{24} X_{52}+2 X_{12} X_{13} X_{24} X_{61} \text {, } \\
& \left.-2 X_{13} X_{21} X_{32}-2 X_{13} X_{24} X_{51}\right)= \\
& =\mathcal{V}\left(X_{11}, X_{22}, X_{33}, X_{34}, X_{43}, X_{25}, X_{42}, X_{23}, X_{41}, X_{15}, X_{13}\right. \text {, } \\
& -\left(X_{21} X_{32}+X_{24} X_{51}\right)^{2}+X_{24} X_{52}\left(2 X_{21} X_{31}+X_{24} X_{61}\right), 2 X_{12} X_{21}+X_{16} X_{61} \text {, } \\
& \left.\left(X_{12} X_{21}\right)^{2}+X_{16} X_{21}^{2} X_{52}\right) \cup \\
& \cup \mathcal{V}\left(X_{11}, X_{22}, X_{33}, X_{34}, X_{43}, X_{25}, X_{42}, X_{23}, X_{41}, X_{15}, X_{21} X_{32}+X_{24} X_{51}\right. \text {, } \\
& X_{24} X_{52}\left(2 X_{21} X_{31}+X_{24} X_{61}\right), 2 X_{12} X_{21}+2 X_{13} X_{31}+X_{16} X_{61} \text {, } \\
& \left.\left(X_{12} X_{21}+X_{13} X_{31}\right)^{2}+X_{16} X_{21}^{2} X_{52}-2 X_{13} X_{21} X_{24} X_{52}+2 X_{12} X_{13} X_{24} X_{61}\right)= \\
& =\mathcal{V}\left(X_{11}, X_{22}, X_{33}, X_{34}, X_{43}, X_{25}, X_{42}, X_{23}, X_{41}, X_{15}, X_{13}\right. \text {, } \\
& -\left(X_{21} X_{32}+X_{24} X_{51}\right)^{2}+X_{24} X_{52}\left(2 X_{21} X_{31}+X_{24} X_{61}\right), 2 X_{12} X_{21}+X_{16} X_{61} \text {, } \\
& \left.\left(X_{12} X_{21}\right)^{2}+X_{16} X_{21}^{2} X_{52}\right) \cup \\
& \cup \mathcal{V}\left(X_{11}, X_{22}, X_{33}, X_{34}, X_{43}, X_{25}, X_{42}, X_{23}, X_{41}, X_{15}, X_{24}, X_{21}, X_{13} X_{31}, X_{16} X_{61}\right) \cup \\
& \cup \mathcal{V}\left(X_{11}, X_{22}, X_{33}, X_{34}, X_{43}, X_{25}, X_{42}, X_{23}, X_{41}, X_{15}, X_{24}, X_{32}\right. \text {, } \\
& \left.2 X_{12} X_{21}+2 X_{13} X_{31}+X_{16} X_{61},\left(X_{12} X_{21}+X_{13} X_{31}\right)^{2}+X_{16} X_{21}^{2} X_{52}\right) \cup \\
& \cup \mathcal{V}\left(X_{11}, X_{22}, X_{33}, X_{34}, X_{43}, X_{25}, X_{42}, X_{23}, X_{41}, X_{15}, X_{52}, X_{21} X_{32}+X_{24} X_{51}\right. \text {, } \\
& \left.2 X_{12} X_{21}+2 X_{13} X_{31}+X_{16} X_{61},\left(X_{12} X_{21}+X_{13} X_{31}\right)^{2}+2 X_{12} X_{13} X_{24} X_{61}\right) \cup \\
& \cup \mathcal{V}\left(X_{11}, X_{22}, X_{33}, X_{34}, X_{43}, X_{25}, X_{42}, X_{23}, X_{41}, X_{15}, 2 X_{21} X_{31}+X_{24} X_{61}\right. \text {, } \\
& X_{21} X_{32}+X_{24} X_{51}, 2 X_{12} X_{21}+2 X_{13} X_{31}+X_{16} X_{61} \text {, } \\
& \left.\left(X_{12} X_{21}+X_{13} X_{31}\right)^{2}+X_{16} X_{21}^{2} X_{52}-2 X_{13} X_{21} X_{24} X_{52}+2 X_{12} X_{13} X_{24} X_{61}\right)
\end{aligned}
$$

As variedades (4.37) e (4.40) são equidimensionais de dimensão 7 pois as

$$
\begin{aligned}
& \mathcal{V}\left(X_{11}, X_{22}, X_{33}, X_{34}, X_{43}, X_{25}, X_{42}, X_{23}, X_{41}, X_{15}, X_{13},\right. \\
& -\left(X_{21} X_{32}+X_{24} X_{51}\right)^{2}+X_{24} X_{52}\left(2 X_{21} X_{31}+X_{24} X_{61}\right), 2 X_{12} X_{21}+X_{16} X_{61}, \\
& \left.\left(X_{12} X_{21}\right)^{2}+X_{16} X_{21}^{2} X_{52}, X_{52}, X_{32}\right)= \\
& =\mathcal{V}\left(X_{11}, X_{22}, X_{33}, X_{34}, X_{43}, X_{25}, X_{42}, X_{23}, X_{41}, X_{15}, X_{13}, X_{52}, X_{32}, X_{24} X_{51}, X_{12} X_{21},\right. \\
& \left.X_{16} X_{61}\right)
\end{aligned}
$$

e

$\mathcal{V}\left(X_{11}, X_{22}, X_{33}, X_{34}, X_{43}, X_{25}, X_{42}, X_{23}, X_{41}, X_{15}, X_{52}, X_{21} X_{32}+X_{24} X_{51}\right.$,

$\left.2 X_{12} X_{21}+2 X_{13} X_{31}+X_{16} X_{61},\left(X_{12} X_{21}+X_{13} X_{31}\right)^{2}+2 X_{12} X_{13} X_{24} X_{61}, X_{32}, X_{12}\right)=$ $=\mathcal{V}\left(X_{11}, X_{22}, X_{33}, X_{34}, X_{43}, X_{25}, X_{42}, X_{23}, X_{41}, X_{15}, X_{52}, X_{32}, X_{12}, X_{24} X_{51}, X_{13} X_{31}\right.$, $\left.X_{16} X_{61}\right)$

são equidimensionais de dimensão $21-16=5$. 
A variedade (4.39) é equidimensional de dimensão 7 pois a variedade

$$
\begin{aligned}
& \mathcal{V}\left(X_{11}, X_{22}, X_{33}, X_{34}, X_{43}, X_{25}, X_{42}, X_{23}, X_{41}, X_{15}, X_{24}, X_{32},\right. \\
& \left.2 X_{12} X_{21}+2 X_{13} X_{31}+X_{16} X_{61},\left(X_{12} X_{21}+X_{13} X_{31}\right)^{2}+X_{16} X_{21}^{2} X_{52}, X_{21}\right)= \\
& =\mathcal{V}\left(X_{11}, X_{22}, X_{33}, X_{34}, X_{43}, X_{25}, X_{42}, X_{23}, X_{41}, X_{15}, X_{24}, X_{32}, X_{21}, X_{13} X_{31}, X_{16} X_{61}\right)
\end{aligned}
$$

é equidimensional de dimensão $21-15=6$.

A variedade (4.41) é equidimensional de dimensão 7 pois a variedade:

$\mathcal{V}\left(X_{11}, X_{22}, X_{33}, X_{34}, X_{43}, X_{25}, X_{42}, X_{23}, X_{41}, X_{15}, 2 X_{21} X_{31}+X_{24} X_{61}\right.$,

$X_{21} X_{32}+X_{24} X_{51}, 2 X_{12} X_{21}+2 X_{13} X_{31}+X_{16} X_{61}$,

$\left.\left(X_{12} X_{21}+X_{13} X_{31}\right)^{2}+X_{16} X_{21}^{2} X_{52}-2 X_{13} X_{21} X_{24} X_{52}+2 X_{12} X_{13} X_{24} X_{61}, X_{32}, X_{52}\right)=$

$=\mathcal{V}\left(X_{11}, X_{22}, X_{33}, X_{34}, X_{43}, X_{25}, X_{42}, X_{23}, X_{41}, X_{15}, X_{32}, X_{52}, X_{24}, X_{21}, X_{13} X_{31}, X_{16} X_{61}\right) \cup$

$\cup \mathcal{V}\left(X_{11}, X_{22}, X_{33}, X_{34}, X_{43}, X_{25}, X_{42}, X_{23}, X_{41}, X_{15}, X_{32}, X_{52}, X_{24}, X_{31}, X_{12} X_{21}, X_{16} X_{61}\right) \cup$

$\cup \mathcal{V}\left(X_{11}, X_{22}, X_{33}, X_{34}, X_{43}, X_{25}, X_{42}, X_{23}, X_{41}, X_{15}, X_{32}, X_{52}, X_{51}, 2 X_{21} X_{31}+X_{24} X_{61}\right.$,

$\left.2 X_{12} X_{21}+2 X_{13} X_{31}+X_{16} X_{61},\left(X_{12} X_{21}+X_{13} X_{31}\right)^{2}+2 X_{12} X_{13} X_{24} X_{61}\right)$

é equidimensional de dimensão 5 já que a variedade abaixo é equidimensional de dimensão $21-17=4$ :

$$
\begin{aligned}
& \mathcal{V}\left(X_{11}, X_{22}, X_{33}, X_{34}, X_{43}, X_{25}, X_{42}, X_{23}, X_{41}, X_{15}, X_{32}, X_{52}, X_{51}, 2 X_{21} X_{31}+X_{24} X_{61},\right. \\
& \left.X_{24} X_{51}, 2 X_{12} X_{21}+2 X_{13} X_{31}+X_{16} X_{61},\left(X_{12} X_{21}+X_{13} X_{31}\right)^{2}+2 X_{12} X_{13} X_{24} X_{61}, X_{24}\right)= \\
& =\mathcal{V}\left(X_{11}, X_{22}, X_{33}, X_{34}, X_{43}, X_{25}, X_{42}, X_{23}, X_{41}, X_{15}, X_{32}, X_{52}, X_{51}, X_{24}, X_{21}, X_{13} X_{31},\right. \\
& \left.X_{16} X_{61}\right) \cup \\
& \cup \mathcal{V}\left(X_{11}, X_{22}, X_{33}, X_{34}, X_{43}, X_{25}, X_{42}, X_{23}, X_{41}, X_{15}, X_{32}, X_{52}, X_{51}, X_{24}, X_{31}, X_{12} X_{21},\right. \\
& \left.X_{16} X_{61}\right)
\end{aligned}
$$

Então a variedade (4.36) acrescida de $X_{15}$ é equidimensional de dimensão 7 e a variedade (4.36) é equidimensional de dimensão 8.

Portanto, a variedade $\mathrm{H}_{2}$ é equidimensional de dimensão 8.

- $\mathrm{H}_{3}$

$$
H_{3}=\mathcal{V}\left(X_{11}, X_{22}, X_{33}, X_{34}, X_{43}, X_{25}, X_{42}, X_{32}, X_{23} X_{31}+X_{24} X_{41}, A, \phi_{6}^{(0)}, \phi_{4}^{(0)}, \partial_{X_{11}} \phi_{4}^{(0)}\right)
$$

Calculando o determinante da matriz $F$ do exemplo 4.3.3 para

$X_{22}=X_{33}=X_{34}=X_{43}=X_{25}=X_{42}=X_{32}=X_{23} X_{31}+X_{24} X_{41}=0$, temos (considerando $\left.X_{11}=0\right)$ : 


$$
\begin{aligned}
\phi_{6}^{(0)}= & -X_{14}^{2} X_{23}^{2} X_{51}^{2}+2 X_{13} X_{14} X_{23} X_{24} X_{51}^{2}-X_{13}^{2} X_{24}^{2} X_{51}^{2}+2 X_{13}^{2} X_{21} X_{24} X_{31} X_{52}- \\
& -2 X_{14}^{2} X_{21} X_{23} X_{41} X_{52}-2 X_{13} X_{14} X_{21} X_{23} X_{31} X_{52}+2 X_{13} X_{14} X_{21} X_{24} X_{41} X_{52}+ \\
& +X_{14}^{2} X_{23}^{2} X_{52} X_{61}-2 X_{13} X_{14} X_{23} X_{24} X_{52} X_{61}+X_{13}^{2} X_{24}^{2} X_{52} X_{61}= \\
& =\left(X_{14} X_{23}-X_{13} X_{24}\right)\left[-X_{51}^{2}\left(X_{14} X_{23}-X_{13} X_{24}\right)+\right. \\
& \left.+X_{52}\left(-2 X_{13} X_{21} X_{31}-2 X_{14} X_{21} X_{41}+X_{14} X_{23} X_{61}-X_{13} X_{24} X_{61}\right)\right] \\
\phi_{4}^{(0)}= & X_{12}^{2} X_{21}^{2}+2 X_{12} X_{13} X_{21} X_{31}+X_{13}^{2} X_{31}^{2}+2 X_{12} X_{14} X_{21} X_{41}+ \\
& +2 X_{13} X_{14} X_{31} X_{41}+X_{14}^{2} X_{41}^{2}+2 X_{12} X_{15} X_{21} X_{51}+2 X_{13} X_{15} X_{31} X_{51}+ \\
& +2 X_{14} X_{15} X_{41} X_{51}+X_{15}^{2} X_{51}^{2}+X_{16} X_{21}^{2} X_{52}+2 X_{14} X_{21} X_{23} X_{52}- \\
& -2 X_{13} X_{21} X_{24} X_{52}-2 X_{12} X_{14} X_{23} X_{61}+2 X_{12} X_{13} X_{24} X_{61}-X_{15}^{2} X_{52} X_{61}= \\
& =\left(X_{12} X_{21}+X_{13} X_{31}+X_{14} X_{41}+X_{15} X_{51}\right)^{2}+X_{16} X_{21}^{2} X_{52}+2 X_{14} X_{21} X_{23} X_{52}- \\
& -2 X_{13} X_{21} X_{24} X_{52}-2 X_{12} X_{14} X_{23} X_{61}+2 X_{12} X_{13} X_{24} X_{61}-X_{15}^{2} X_{52} X_{61}
\end{aligned}
$$

$\partial_{X_{11}} \phi_{4}^{(0)}=+2 X_{14} X_{23} X_{51}-2 X_{13} X_{24} X_{51}-2 X_{15} X_{21} X_{52}$

E $A=2 X_{12} X_{21}+2 X_{13} X_{31}+2 X_{14} X_{41}+2 X_{15} X_{51}+X_{16} X_{61}$.

Por $\phi_{6}^{(0)}$ temos que $H_{3}$ é a união das duas variedades abaixo:

$$
\begin{aligned}
& \mathcal{V}\left(X_{11}, X_{22}, X_{33}, X_{34}, X_{43}, X_{25}, X_{42}, X_{32}, X_{23} X_{31}+X_{24} X_{41}, X_{14} X_{23}-X_{13} X_{24}, A, \phi_{4}^{(0)},\right. \\
& \left.\partial_{X_{11}} \phi_{4}^{(0)}\right)
\end{aligned}
$$

e

$$
\begin{aligned}
& \mathcal{V}\left(X_{11}, X_{22}, X_{33}, X_{34}, X_{43}, X_{25}, X_{42}, X_{32}, X_{23} X_{31}+X_{24} X_{41},\right. \\
& -X_{51}^{2}\left(X_{14} X_{23}-X_{13} X_{24}\right)+X_{52}\left(-2 X_{13} X_{21} X_{31}-2 X_{14} X_{21} X_{41}+X_{14} X_{23} X_{61}-X_{13} X_{24} X_{61}\right) \\
& \left.A, \phi_{4}^{(0)}, \partial_{X_{11}} \phi_{4}^{(0)}\right)
\end{aligned}
$$


A variedade (4.42) acrescida de $X_{13}$ é igual a:

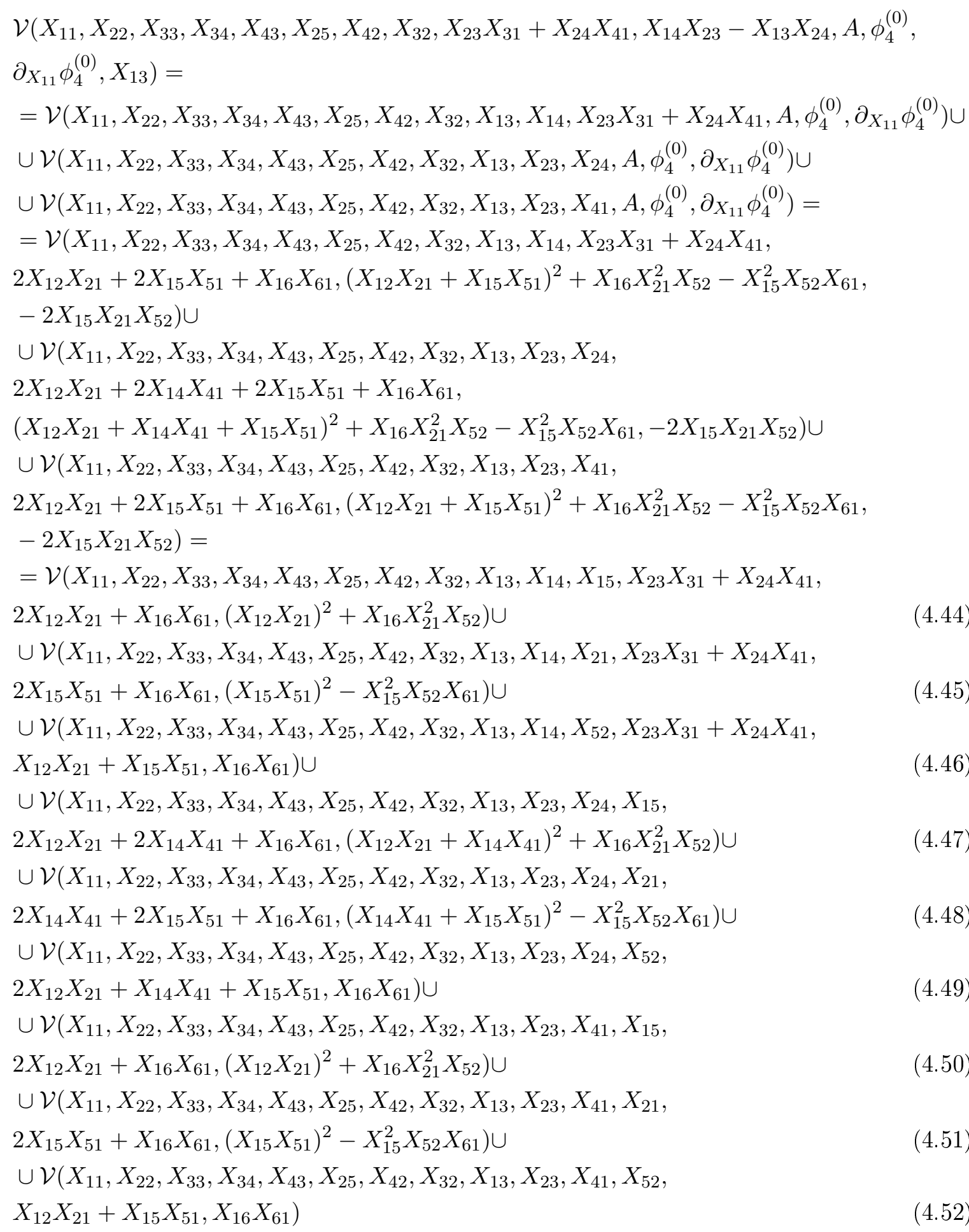

A variedade (4.44) é equidimensional de dimensão 7 pois a variedade

$$
\begin{aligned}
& \mathcal{V}\left(X_{11}, X_{22}, X_{33}, X_{34}, X_{43}, X_{25}, X_{42}, X_{32}, X_{13}, X_{14}, X_{15}, X_{23} X_{31}+X_{24} X_{41},\right. \\
& \left.2 X_{12} X_{21}+X_{16} X_{61},\left(X_{12} X_{21}\right)^{2}+X_{16} X_{21}^{2} X_{52}, X_{52}, X_{24}\right)= \\
& =\mathcal{V}\left(X_{11}, X_{22}, X_{33}, X_{34}, X_{43}, X_{25}, X_{42}, X_{32}, X_{13}, X_{14}, X_{15}, X_{52}, X_{24}, X_{23} X_{31},\right. \\
& \left.X_{12} X_{21}, X_{16} X_{61}\right)
\end{aligned}
$$

é equidimensional de dimensão $21-16=5$. 
A variedade (4.45) é equidimensional de dimensão 7 pois a variedade

$$
\begin{aligned}
& \mathcal{V}\left(X_{11}, X_{22}, X_{33}, X_{34}, X_{43}, X_{25}, X_{42}, X_{32}, X_{13}, X_{14}, X_{21}, X_{23} X_{31}+X_{24} X_{41},\right. \\
& \left.2 X_{15} X_{51}+X_{16} X_{61},\left(X_{15} X_{51}\right)^{2}-X_{15}^{2} X_{52} X_{61}, X_{52}, X_{24}\right)= \\
& =\mathcal{V}\left(X_{11}, X_{22}, X_{33}, X_{34}, X_{43}, X_{25}, X_{42}, X_{32}, X_{13}, X_{14}, X_{21}, X_{52}, X_{24}, X_{23} X_{31},\right. \\
& \left.X_{15} X_{51}, X_{16} X_{61}\right)
\end{aligned}
$$

é equidimensional de dimensão $21-16=5$.

A variedade (4.46) é equidimensional de dimensão 7 pois a variedade

$$
\begin{aligned}
& \mathcal{V}\left(X_{11}, X_{22}, X_{33}, X_{34}, X_{43}, X_{25}, X_{42}, X_{32}, X_{13}, X_{14}, X_{52}, X_{23} X_{31}+X_{24} X_{41},\right. \\
& \left.X_{12} X_{21}+X_{15} X_{51}, X_{16} X_{61}, X_{12}, X_{24}\right)= \\
& =\mathcal{V}\left(X_{11}, X_{22}, X_{33}, X_{34}, X_{43}, X_{25}, X_{42}, X_{32}, X_{13}, X_{14}, X_{52}, X_{12}, X_{24}, X_{23} X_{31},\right. \\
& \left.X_{15} X_{51}, X_{16} X_{61}\right)
\end{aligned}
$$

é equidimensional de dimensão $21-16=5$.

A variedade (4.47) é equidimensional de dimensão 7 pois a variedade

$$
\begin{aligned}
& \mathcal{V}\left(X_{11}, X_{22}, X_{33}, X_{34}, X_{43}, X_{25}, X_{42}, X_{32}, X_{13}, X_{23}, X_{24}, X_{15},\right. \\
& \left.2 X_{12} X_{21}+2 X_{14} X_{41}+X_{16} X_{61},\left(X_{12} X_{21}+X_{14} X_{41}\right)^{2}+X_{16} X_{21}^{2} X_{52}, X_{21}\right)= \\
& =\mathcal{V}\left(X_{11}, X_{22}, X_{33}, X_{34}, X_{43}, X_{25}, X_{42}, X_{32}, X_{13}, X_{23}, X_{24}, X_{15}, X_{21}, X_{14} X_{41}, X_{16} X_{61}\right)
\end{aligned}
$$

é equidimensional de dimensão $21-15=6$.

A variedade (4.48) é equidimensional de dimensão 7 pois a variedade

$$
\begin{aligned}
& \mathcal{V}\left(X_{11}, X_{22}, X_{33}, X_{34}, X_{43}, X_{25}, X_{42}, X_{32}, X_{13}, X_{23}, X_{24}, X_{21},\right. \\
& \left.2 X_{14} X_{41}+2 X_{15} X_{51}+X_{16} X_{61},\left(X_{14} X_{41}+X_{15} X_{51}\right)^{2}-X_{15}^{2} X_{52} X_{61}, X_{15}\right)= \\
& =\mathcal{V}\left(X_{11}, X_{22}, X_{33}, X_{34}, X_{43}, X_{25}, X_{42}, X_{32}, X_{13}, X_{23}, X_{24}, X_{21}, X_{15}, X_{14} X_{41}, X_{16} X_{61}\right)
\end{aligned}
$$

é equidimensional de dimensão $21-15=6$.

A variedade (4.49) é equidimensional de dimensão 7 pois a variedade

$$
\begin{aligned}
& \mathcal{V}\left(X_{11}, X_{22}, X_{33}, X_{34}, X_{43}, X_{25}, X_{42}, X_{32}, X_{13}, X_{23}, X_{24}, X_{52},\right. \\
& \left.2 X_{12} X_{21}+X_{14} X_{41}+X_{15} X_{51}, X_{16} X_{61}, X_{12}, X_{14}\right)= \\
& =\mathcal{V}\left(X_{11}, X_{22}, X_{33}, X_{34}, X_{43}, X_{25}, X_{42}, X_{32}, X_{13}, X_{23}, X_{24}, X_{52}, X_{12}, X_{14}, X_{15} X_{51}, X_{16} X_{61}\right)
\end{aligned}
$$

é equidimensional de dimensão $21-16=5$.

A variedade (4.50) é equidimensional de dimensão 7 pois a variedade

$$
\begin{aligned}
& \mathcal{V}\left(X_{11}, X_{22}, X_{33}, X_{34}, X_{43}, X_{25}, X_{42}, X_{32}, X_{13}, X_{23}, X_{41}, X_{15},\right. \\
& \left.2 X_{12} X_{21}+X_{16} X_{61},\left(X_{12} X_{21}\right)^{2}+X_{16} X_{21}^{2} X_{52}, X_{52}\right)= \\
& =\mathcal{V}\left(X_{11}, X_{22}, X_{33}, X_{34}, X_{43}, X_{25}, X_{42}, X_{32}, X_{13}, X_{23}, X_{41}, X_{15}, X_{52}, X_{12} X_{21}, X_{16} X_{61}\right)
\end{aligned}
$$

é equidimensional de dimensão $21-15=6$.

A variedade (4.51) é equidimensional de dimensão 7 pois a variedade

$$
\begin{aligned}
& \mathcal{V}\left(X_{11}, X_{22}, X_{33}, X_{34}, X_{43}, X_{25}, X_{42}, X_{32}, X_{13}, X_{23}, X_{41}, X_{21},\right. \\
& \left.2 X_{15} X_{51}+X_{16} X_{61},\left(X_{15} X_{51}\right)^{2}-X_{15}^{2} X_{52} X_{61}, X_{52}\right)= \\
& =\mathcal{V}\left(X_{11}, X_{22}, X_{33}, X_{34}, X_{43}, X_{25}, X_{42}, X_{32}, X_{13}, X_{23}, X_{41}, X_{21}, X_{52}, X_{15} X_{51}, X_{16} X_{61}\right)
\end{aligned}
$$

é equidimensional de dimensão $21-15=6$. 
A variedade (4.52) é equidimensional de dimensão 7 pois a variedade

$$
\begin{aligned}
& \mathcal{V}\left(X_{11}, X_{22}, X_{33}, X_{34}, X_{43}, X_{25}, X_{42}, X_{32}, X_{13}, X_{23}, X_{41}, X_{52},\right. \\
& \left.X_{12} X_{21}+X_{15} X_{51}, X_{16} X_{61}, X_{12}\right)= \\
& =\mathcal{V}\left(X_{11}, X_{22}, X_{33}, X_{34}, X_{43}, X_{25}, X_{42}, X_{32}, X_{13}, X_{23}, X_{41}, X_{52}, X_{12}, X_{15} X_{51}, X_{16} X_{61}\right)
\end{aligned}
$$

é equidimensional de dimensão $21-15=6$.

Então a variedade (4.42) acrescida de $X_{13}$ é equidimensional de dimensão 7 e a variedade (4.42) é equidimensional de dimensão 8. 
Considere agora a variedade (4.43):

$\mathcal{V}\left(X_{11}, X_{22}, X_{33}, X_{34}, X_{43}, X_{25}, X_{42}, X_{32}, X_{23} X_{31}+X_{24} X_{41}\right.$,

$-X_{51}^{2}\left(X_{14} X_{23}-X_{13} X_{24}\right)+X_{52}\left(-2 X_{13} X_{21} X_{31}-2 X_{14} X_{21} X_{41}+X_{14} X_{23} X_{61}-X_{13} X_{24} X_{61}\right)$, $\left.A, \phi_{6}^{(0)}, \phi_{4}^{(0)}, \partial_{X_{11}} \phi_{4}^{(0)}\right)=$

$=\mathcal{V}\left(X_{11}, X_{22}, X_{33}, X_{34}, X_{43}, X_{25}, X_{42}, X_{32}, X_{23} X_{31}+X_{24} X_{41}\right.$,

$-X_{51}^{2}\left(X_{14} X_{23}-X_{13} X_{24}\right)+X_{52}\left(-2 X_{13} X_{21} X_{31}-2 X_{14} X_{21} X_{41}+X_{14} X_{23} X_{61}-X_{13} X_{24} X_{61}\right)$,

$2 X_{12} X_{21}+2 X_{13} X_{31}+2 X_{14} X_{41}+2 X_{15} X_{51}+X_{16} X_{61}$,

$\left(X_{12} X_{21}+X_{13} X_{31}+X_{14} X_{41}+X_{15} X_{51}\right)^{2}+X_{16} X_{21}^{2} X_{52}+2 X_{14} X_{21} X_{23} X_{52}-$

$-2 X_{13} X_{21} X_{24} X_{52}-2 X_{12} X_{14} X_{23} X_{61}+2 X_{12} X_{13} X_{24} X_{61}-X_{15}^{2} X_{52} X_{61}$,

$\left.2 X_{14} X_{23} X_{51}-2 X_{13} X_{24} X_{51}-2 X_{15} X_{21} X_{52}\right)=$

$=\mathcal{V}\left(X_{11}, X_{22}, X_{33}, X_{34}, X_{43}, X_{25}, X_{42}, X_{32}, X_{23} X_{31}+X_{24} X_{41}\right.$,

$-X_{51} X_{15} X_{21} X_{52}+X_{52}\left(-2 X_{13} X_{21} X_{31}-2 X_{14} X_{21} X_{41}+X_{14} X_{23} X_{61}-X_{13} X_{24} X_{61}\right)$,

$2 X_{12} X_{21}+2 X_{13} X_{31}+2 X_{14} X_{41}+2 X_{15} X_{51}+X_{16} X_{61}$,

$\left(X_{12} X_{21}+X_{13} X_{31}+X_{14} X_{41}+X_{15} X_{51}\right)^{2}+X_{16} X_{21}^{2} X_{52}+2 X_{14} X_{21} X_{23} X_{52}-$

$-2 X_{13} X_{21} X_{24} X_{52}-2 X_{12} X_{14} X_{23} X_{61}+2 X_{12} X_{13} X_{24} X_{61}-X_{15}^{2} X_{52} X_{61}$,

$\left.2 X_{14} X_{23} X_{51}-2 X_{13} X_{24} X_{51}-2 X_{15} X_{21} X_{52}\right)=$

$=\mathcal{V}\left(X_{11}, X_{22}, X_{33}, X_{34}, X_{43}, X_{25}, X_{42}, X_{32}, X_{52}, X_{23} X_{31}+X_{24} X_{41}\right.$,

$2 X_{12} X_{21}+2 X_{13} X_{31}+2 X_{14} X_{41}+2 X_{15} X_{51}+X_{16} X_{61}$,

$\left(X_{12} X_{21}+X_{13} X_{31}+X_{14} X_{41}+X_{15} X_{51}\right)^{2}-2 X_{12} X_{14} X_{23} X_{61}+2 X_{12} X_{13} X_{24} X_{61}$,

$\left.2 X_{14} X_{23} X_{51}-2 X_{13} X_{24} X_{51}\right) \cup$

$\cup \mathcal{V}\left(X_{11}, X_{22}, X_{33}, X_{34}, X_{43}, X_{25}, X_{42}, X_{32}, X_{23} X_{31}+X_{24} X_{41}\right.$,

$-X_{51} X_{15} X_{21}+-2 X_{13} X_{21} X_{31}-2 X_{14} X_{21} X_{41}+X_{14} X_{23} X_{61}-X_{13} X_{24} X_{61}$,

$2 X_{12} X_{21}+2 X_{13} X_{31}+2 X_{14} X_{41}+2 X_{15} X_{51}+X_{16} X_{61}$,

$\left(X_{12} X_{21}+X_{13} X_{31}+X_{14} X_{41}+X_{15} X_{51}\right)^{2}+X_{16} X_{21}^{2} X_{52}+2 X_{14} X_{21} X_{23} X_{52}-$

$-2 X_{13} X_{21} X_{24} X_{52}-2 X_{12} X_{14} X_{23} X_{61}+2 X_{12} X_{13} X_{24} X_{61}-X_{15}^{2} X_{52} X_{61}$,

$\left.2 X_{14} X_{23} X_{51}-2 X_{13} X_{24} X_{51}-2 X_{15} X_{21} X_{52}\right)=$

$=\mathcal{V}\left(X_{11}, X_{22}, X_{33}, X_{34}, X_{43}, X_{25}, X_{42}, X_{32}, X_{52}, X_{51}, X_{23} X_{31}+X_{24} X_{41}\right.$,

$2 X_{12} X_{21}+2 X_{13} X_{31}+2 X_{14} X_{41}+X_{16} X_{61}$,

$\left.\left(X_{12} X_{21}+X_{13} X_{31}+X_{14} X_{41}\right)^{2}-2 X_{12} X_{14} X_{23} X_{61}+2 X_{12} X_{13} X_{24} X_{61}\right) \cup$

$\cup \mathcal{V}\left(X_{11}, X_{22}, X_{33}, X_{34}, X_{43}, X_{25}, X_{42}, X_{32}, X_{52}, X_{14} X_{23}-X_{13} X_{24}, X_{23} X_{31}+X_{24} X_{41}\right.$,

$\left.X_{12} X_{21}+X_{13} X_{31}+X_{14} X_{41}+X_{15} X_{51}, X_{16} X_{61}\right) \cup$

$\cup \mathcal{V}\left(X_{11}, X_{22}, X_{33}, X_{34}, X_{43}, X_{25}, X_{42}, X_{32}, X_{23} X_{31}+X_{24} X_{41}\right.$,

$-X_{51} X_{15} X_{21}+-2 X_{13} X_{21} X_{31}-2 X_{14} X_{21} X_{41}+X_{14} X_{23} X_{61}-X_{13} X_{24} X_{61}$,

$2 X_{12} X_{21}+2 X_{13} X_{31}+2 X_{14} X_{41}+2 X_{15} X_{51}+X_{16} X_{61}$,

$\left(X_{12} X_{21}+X_{13} X_{31}+X_{14} X_{41}+X_{15} X_{51}\right)^{2}+X_{16} X_{21}^{2} X_{52}+2 X_{14} X_{21} X_{23} X_{52}-$

$-2 X_{13} X_{21} X_{24} X_{52}-2 X_{12} X_{14} X_{23} X_{61}+2 X_{12} X_{13} X_{24} X_{61}-X_{15}^{2} X_{52} X_{61}$,

$\left.2 X_{14} X_{23} X_{51}-2 X_{13} X_{24} X_{51}-2 X_{15} X_{21} X_{52}\right)$ 
A variedade (4.53) acrescida de $X_{24}$ é igual a

$$
\begin{aligned}
& \mathcal{V}\left(X_{11}, X_{22}, X_{33}, X_{34}, X_{43}, X_{25}, X_{42}, X_{32}, X_{52}, X_{51}, X_{23} X_{31}+X_{24} X_{41},\right. \\
& 2 X_{12} X_{21}+2 X_{13} X_{31}+2 X_{14} X_{41}+X_{16} X_{61}, \\
& \left.\left(X_{12} X_{21}+X_{13} X_{31}+X_{14} X_{41}\right)^{2}-2 X_{12} X_{14} X_{23} X_{61}+2 X_{12} X_{13} X_{24} X_{61}, X_{24}\right)= \\
& =\mathcal{V}\left(X_{11}, X_{22}, X_{33}, X_{34}, X_{43}, X_{25}, X_{42}, X_{32}, X_{52}, X_{51}, X_{24}, X_{23} X_{31},\right. \\
& \left.2 X_{12} X_{21}+2 X_{13} X_{31}+2 X_{14} X_{41}+X_{16} X_{61},\left(X_{12} X_{21}+X_{13} X_{31}+X_{14} X_{41}\right)^{2}-2 X_{12} X_{14} X_{23} X_{61}\right)= \\
& =\mathcal{V}\left(X_{11}, X_{22}, X_{33}, X_{34}, X_{43}, X_{25}, X_{42}, X_{32}, X_{52}, X_{51}, X_{24}, X_{23},\right. \\
& \left.X_{12} X_{21}+X_{13} X_{31}+X_{14} X_{41}, X_{16} X_{61}\right) \cup \\
& \cup \mathcal{V}\left(X_{11}, X_{22}, X_{33}, X_{34}, X_{43}, X_{25}, X_{42}, X_{32}, X_{52}, X_{51}, X_{24}, X_{31},\right. \\
& \left.2 X_{12} X_{21}+2 X_{14} X_{41}+X_{16} X_{61},\left(X_{12} X_{21}+X_{14} X_{41}\right)^{2}-2 X_{12} X_{14} X_{23} X_{61}\right)
\end{aligned}
$$

A variedade

$$
\begin{aligned}
& \mathcal{V}\left(X_{11}, X_{22}, X_{33}, X_{34}, X_{43}, X_{25}, X_{42}, X_{32}, X_{52}, X_{51}, X_{24}, X_{23},\right. \\
& \left.X_{12} X_{21}+X_{13} X_{31}+X_{14} X_{41}, X_{16} X_{61}, X_{41}, X_{31}\right)= \\
& =\mathcal{V}\left(X_{11}, X_{22}, X_{33}, X_{34}, X_{43}, X_{25}, X_{42}, X_{32}, X_{52}, X_{51}, X_{24}, X_{23}, X_{41}, X_{31},\right. \\
& \left.X_{12} X_{21}, X_{16} X_{61}\right)
\end{aligned}
$$

é equidimensional de dimensão $21-16=5$ e a variedade

$$
\begin{aligned}
& \mathcal{V}\left(X_{11}, X_{22}, X_{33}, X_{34}, X_{43}, X_{25}, X_{42}, X_{32}, X_{52}, X_{51}, X_{24}, X_{31},\right. \\
& \left.2 X_{12} X_{21}+2 X_{14} X_{41}+X_{16} X_{61},\left(X_{12} X_{21}+X_{14} X_{41}\right)^{2}-2 X_{12} X_{14} X_{23} X_{61}, X_{14}\right)= \\
& =\mathcal{V}\left(X_{11}, X_{22}, X_{33}, X_{34}, X_{43}, X_{25}, X_{42}, X_{32}, X_{52}, X_{51}, X_{24}, X_{31}, X_{14},\right. \\
& \left.X_{12} X_{21}, X_{16} X_{61}\right)
\end{aligned}
$$

é equidimensional de dimensão $21-15=6$. Então a variedade (4.53) acrescida de $X_{24}$ é equidimensional de dimensão 7 e a variedade (4.53) é equidimensional de dimensão 8.

A variedade (4.54) acrescida de $X_{41}$ é igual a:

$$
\begin{aligned}
& \mathcal{V}\left(X_{11}, X_{22}, X_{33}, X_{34}, X_{43}, X_{25}, X_{42}, X_{32}, X_{52}, X_{14} X_{23}-X_{13} X_{24}, X_{23} X_{31}+X_{24} X_{41},\right. \\
& \left.X_{12} X_{21}+X_{13} X_{31}+X_{14} X_{41}+X_{15} X_{51}, X_{16} X_{61}, X_{41}\right)= \\
& =\mathcal{V}\left(X_{11}, X_{22}, X_{33}, X_{34}, X_{43}, X_{25}, X_{42}, X_{32}, X_{52}, X_{41}, X_{14} X_{23}-X_{13} X_{24}, X_{23} X_{31},\right. \\
& \left.X_{12} X_{21}+X_{13} X_{31}+X_{15} X_{51}, X_{16} X_{61}\right)= \\
& =\mathcal{V}\left(X_{11}, X_{22}, X_{33}, X_{34}, X_{43}, X_{25}, X_{42}, X_{32}, X_{52}, X_{41}, X_{23}, X_{13} X_{24},\right. \\
& \left.X_{12} X_{21}+X_{13} X_{31}+X_{15} X_{51}, X_{16} X_{61}\right) \cup \\
& \cup \mathcal{V}\left(X_{11}, X_{22}, X_{33}, X_{34}, X_{43}, X_{25}, X_{42}, X_{32}, X_{52}, X_{41}, X_{31}, X_{14} X_{23}-X_{13} X_{24},\right. \\
& \left.X_{12} X_{21}+X_{15} X_{51}, X_{16} X_{61}\right)= \\
& =\mathcal{V}\left(X_{11}, X_{22}, X_{33}, X_{34}, X_{43}, X_{25}, X_{42}, X_{32}, X_{52}, X_{41}, X_{23}, X_{13},\right. \\
& \left.X_{12} X_{21}+X_{15} X_{51}, X_{16} X_{61}\right) \cup \\
& \cup \mathcal{V}\left(X_{11}, X_{22}, X_{33}, X_{34}, X_{43}, X_{25}, X_{42}, X_{32}, X_{52}, X_{41}, X_{23}, X_{24},\right. \\
& \left.X_{12} X_{21}+X_{13} X_{31}+X_{15} X_{51}, X_{16} X_{61}\right) \cup \\
& \cup \mathcal{V}\left(X_{11}, X_{22}, X_{33}, X_{34}, X_{43}, X_{25}, X_{42}, X_{32}, X_{52}, X_{41}, X_{31}, X_{14} X_{23}-X_{13} X_{24},\right. \\
& \left.X_{12} X_{21}+X_{15} X_{51}, X_{16} X_{61}\right)
\end{aligned}
$$


A variedade

$$
\begin{aligned}
& \mathcal{V}\left(X_{11}, X_{22}, X_{33}, X_{34}, X_{43}, X_{25}, X_{42}, X_{32}, X_{52}, X_{41}, X_{23}, X_{13}\right. \\
& \left.X_{12} X_{21}+X_{15} X_{51}, X_{16} X_{61}, X_{12}\right)= \\
& =\mathcal{V}\left(X_{11}, X_{22}, X_{33}, X_{34}, X_{43}, X_{25}, X_{42}, X_{32}, X_{52}, X_{41}, X_{23}, X_{13}, X_{12}, X_{15} X_{51}, X_{16} X_{61}\right)
\end{aligned}
$$

é equidimensional de dimensão $21-15=6$ e as variedades abaixo são equidimensionais de dimensão $21-16=5$ :

$$
\begin{aligned}
& \quad \mathcal{V}\left(X_{11}, X_{22}, X_{33}, X_{34}, X_{43}, X_{25}, X_{42}, X_{32}, X_{52}, X_{41}, X_{23}, X_{24},\right. \\
& \left.\quad X_{12} X_{21}+X_{13} X_{31}+X_{15} X_{51}, X_{16} X_{61}, X_{13}, X_{12}\right)= \\
& \quad=\mathcal{V}\left(X_{11}, X_{22}, X_{33}, X_{34}, X_{43}, X_{25}, X_{42}, X_{32}, X_{52}, X_{41}, X_{23}, X_{24}, X_{13}, X_{12},\right. \\
& \left.\quad X_{15} X_{51}, X_{16} X_{61}\right) \\
& \mathcal{V}\left(X_{11}, X_{22}, X_{33}, X_{34}, X_{43}, X_{25}, X_{42}, X_{32}, X_{52}, X_{41}, X_{31}, X_{14} X_{23}-X_{13} X_{24},\right. \\
& \left.X_{12} X_{21}+X_{15} X_{51}, X_{16} X_{61}, X_{13}, X_{12}\right)= \\
& =\mathcal{V}\left(X_{11}, X_{22}, X_{33}, X_{34}, X_{43}, X_{25}, X_{42}, X_{32}, X_{52}, X_{41}, X_{31}, X_{13}, X_{12}, X_{14} X_{23},\right. \\
& \left.X_{15} X_{51}, X_{16} X_{61}\right)
\end{aligned}
$$

então a variedade (4.54) acrescida de $X_{41}$ é equidimensional de dimensão 7 e (4.54) é equidimensional de dimensão 8. 
A variedade (4.55) acrescida de $X_{52}$ é igual a:

$$
\begin{aligned}
& \mathcal{V}\left(X_{11}, X_{22}, X_{33}, X_{34}, X_{43}, X_{25}, X_{42}, X_{32}, X_{23} X_{31}+X_{24} X_{41}\right. \text {, } \\
& 2 X_{12} X_{21}+2 X_{13} X_{31}+2 X_{14} X_{41}+2 X_{15} X_{51}+X_{16} X_{61} \\
& -X_{15} X_{21} X_{51}-2 X_{13} X_{21} X_{31}-2 X_{14} X_{21} X_{41}+X_{14} X_{23} X_{61}-X_{13} X_{24} X_{61} \text {, } \\
& \left(X_{12} X_{21}+X_{13} X_{31}+X_{14} X_{41}+X_{15} X_{51}\right)^{2}+X_{16} X_{21}^{2} X_{52}+2 X_{14} X_{21} X_{23} X_{52}- \\
& -2 X_{13} X_{21} X_{24} X_{52}-2 X_{12} X_{14} X_{23} X_{61}+2 X_{12} X_{13} X_{24} X_{61}-X_{15}^{2} X_{52} X_{61} \text {, } \\
& \left.2 X_{14} X_{23} X_{51}-2 X_{13} X_{24} X_{51}-2 X_{15} X_{21} X_{52}, X_{52}\right)= \\
& =\mathcal{V}\left(X_{11}, X_{22}, X_{33}, X_{34}, X_{43}, X_{25}, X_{42}, X_{32}, X_{52}, X_{23} X_{31}+X_{24} X_{41}\right. \text {, } \\
& 2 X_{12} X_{21}+2 X_{13} X_{31}+2 X_{14} X_{41}+2 X_{15} X_{51}+X_{16} X_{61} \\
& -X_{15} X_{21} X_{51}-2 X_{13} X_{21} X_{31}-2 X_{14} X_{21} X_{41}+X_{14} X_{23} X_{61}-X_{13} X_{24} X_{61} \text {, } \\
& \left(X_{12} X_{21}+X_{13} X_{31}+X_{14} X_{41}+X_{15} X_{51}\right)^{2}-2 X_{12} X_{14} X_{23} X_{61}+2 X_{12} X_{13} X_{24} X_{61} \text {, } \\
& \left.2 X_{14} X_{23} X_{51}-2 X_{13} X_{24} X_{51}\right)= \\
& =\mathcal{V}\left(X_{11}, X_{22}, X_{33}, X_{34}, X_{43}, X_{25}, X_{42}, X_{32}, X_{52}, X_{51}, X_{23} X_{31}+X_{24} X_{41}\right. \text {, } \\
& 2 X_{12} X_{21}+2 X_{13} X_{31}+2 X_{14} X_{41}+X_{16} X_{61} \text {, } \\
& -2 X_{13} X_{21} X_{31}-2 X_{14} X_{21} X_{41}+X_{14} X_{23} X_{61}-X_{13} X_{24} X_{61} \text {, } \\
& \left.\left(X_{12} X_{21}+X_{13} X_{31}+X_{14} X_{41}\right)^{2}-2 X_{12} X_{14} X_{23} X_{61}+2 X_{12} X_{13} X_{24} X_{61}\right) \cup \\
& \cup \mathcal{V}\left(X_{11}, X_{22}, X_{33}, X_{34}, X_{43}, X_{25}, X_{42}, X_{32}, X_{52}, X_{23} X_{31}+X_{24} X_{41}, X_{14} X_{23}-X_{13} X_{24}\right. \\
& \left.X_{12} X_{21}+X_{13} X_{31}+X_{14} X_{41}+X_{15} X_{51}, X_{16} X_{61},-X_{15} X_{21} X_{51}-2 X_{13} X_{21} X_{31}-2 X_{14} X_{21} X_{41}\right)= \\
& =\mathcal{V}\left(X_{11}, X_{22}, X_{33}, X_{34}, X_{43}, X_{25}, X_{42}, X_{32}, X_{52}, X_{51}, X_{23} X_{31}+X_{24} X_{41}\right. \text {, } \\
& 2 X_{12} X_{21}+2 X_{13} X_{31}+2 X_{14} X_{41}+X_{16} X_{61} \text {, } \\
& -2 X_{13} X_{21} X_{31}-2 X_{14} X_{21} X_{41}+X_{14} X_{23} X_{61}-X_{13} X_{24} X_{61} \text {, } \\
& \left.\left(X_{12} X_{21}+X_{13} X_{31}+X_{14} X_{41}\right)^{2}-2 X_{12} X_{14} X_{23} X_{61}+2 X_{12} X_{13} X_{24} X_{61}\right) \cup \\
& \cup \mathcal{V}\left(X_{11}, X_{22}, X_{33}, X_{34}, X_{43}, X_{25}, X_{42}, X_{32}, X_{52}, X_{21}, X_{23} X_{31}+X_{24} X_{41}, X_{14} X_{23}-X_{13} X_{24}\right. \\
& \left.X_{13} X_{31}+X_{14} X_{41}+X_{15} X_{51}, X_{16} X_{61}\right) \cup \\
& \cup \mathcal{V}\left(X_{11}, X_{22}, X_{33}, X_{34}, X_{43}, X_{25}, X_{42}, X_{32}, X_{52}, X_{23} X_{31}+X_{24} X_{41}, X_{14} X_{23}-X_{13} X_{24}\right. \\
& \left.X_{12} X_{21}+X_{13} X_{31}+X_{14} X_{41}+X_{15} X_{51}, X_{16} X_{61},-X_{15} X_{51}-2 X_{13} X_{31}-2 X_{14} X_{41}\right)
\end{aligned}
$$


A variedade (4.56) acrescida de $X_{23}$ é igual a:

$$
\begin{aligned}
& \mathcal{V}\left(X_{11}, X_{22}, X_{33}, X_{34}, X_{43}, X_{25}, X_{42}, X_{32}, X_{52}, X_{51}, X_{23} X_{31}+X_{24} X_{41}\right. \text {, } \\
& 2 X_{12} X_{21}+2 X_{13} X_{31}+2 X_{14} X_{41}+X_{16} X_{61} \text {, } \\
& -2 X_{13} X_{21} X_{31}-2 X_{14} X_{21} X_{41}+X_{14} X_{23} X_{61}-X_{13} X_{24} X_{61} \text {, } \\
& \left.\left(X_{12} X_{21}+X_{13} X_{31}+X_{14} X_{41}\right)^{2}-2 X_{12} X_{14} X_{23} X_{61}+2 X_{12} X_{13} X_{24} X_{61}, X_{23}\right)= \\
& =\mathcal{V}\left(X_{11}, X_{22}, X_{33}, X_{34}, X_{43}, X_{25}, X_{42}, X_{32}, X_{52}, X_{51}, X_{23}, X_{24} X_{41}\right. \text {, } \\
& 2 X_{12} X_{21}+2 X_{13} X_{31}+2 X_{14} X_{41}+X_{16} X_{61},-2 X_{13} X_{21} X_{31}-2 X_{14} X_{21} X_{41}-X_{13} X_{24} X_{61} \text {, } \\
& \left.\left(X_{12} X_{21}+X_{13} X_{31}+X_{14} X_{41}\right)^{2}+2 X_{12} X_{13} X_{24} X_{61}\right)= \\
& =\mathcal{V}\left(X_{11}, X_{22}, X_{33}, X_{34}, X_{43}, X_{25}, X_{42}, X_{32}, X_{52}, X_{51}, X_{23}, X_{24}\right. \text {, } \\
& \left.X_{12} X_{21}+X_{13} X_{31}+X_{14} X_{41}, X_{16} X_{61},-2 X_{13} X_{21} X_{31}-2 X_{14} X_{21} X_{41}\right) \cup \\
& \cup \mathcal{V}\left(X_{11}, X_{22}, X_{33}, X_{34}, X_{43}, X_{25}, X_{42}, X_{32}, X_{52}, X_{51}, X_{23}, X_{41}\right. \text {, } \\
& 2 X_{12} X_{21}+2 X_{13} X_{31}+X_{16} X_{61},-2 X_{13} X_{21} X_{31}-X_{13} X_{24} X_{61} \text {, } \\
& \left.\left(X_{12} X_{21}+X_{13} X_{31}\right)^{2}+2 X_{12} X_{13} X_{24} X_{61}\right)= \\
& =\mathcal{V}\left(X_{11}, X_{22}, X_{33}, X_{34}, X_{43}, X_{25}, X_{42}, X_{32}, X_{52}, X_{51}, X_{23}, X_{24}, X_{21}\right. \text {, } \\
& \left.X_{13} X_{31}+X_{14} X_{41}, X_{16} X_{61}\right) \cup \\
& \cup \mathcal{V}\left(X_{11}, X_{22}, X_{33}, X_{34}, X_{43}, X_{25}, X_{42}, X_{32}, X_{52}, X_{51}, X_{23}, X_{24}\right. \text {, } \\
& \left.X_{12} X_{21}, X_{13} X_{31}+X_{14} X_{41}, X_{16} X_{61}\right) \cup \\
& \cup \mathcal{V}\left(X_{11}, X_{22}, X_{33}, X_{34}, X_{43}, X_{25}, X_{42}, X_{32}, X_{52}, X_{51}, X_{23}, X_{41}, X_{13}\right. \text {, } \\
& \left.X_{12} X_{21}, X_{16} X_{61}\right) \cup \\
& \cup \mathcal{V}\left(X_{11}, X_{22}, X_{33}, X_{34}, X_{43}, X_{25}, X_{42}, X_{32}, X_{52}, X_{51}, X_{23}, X_{41}, 2 X_{21} X_{31}+X_{24} X_{61}\right. \text {, } \\
& \left.2 X_{12} X_{21}+2 X_{13} X_{31}+X_{16} X_{61},\left(X_{12} X_{21}+X_{13} X_{31}\right)^{2}+2 X_{12} X_{13} X_{24} X_{61}\right)
\end{aligned}
$$

As variedades abaixo são equidimensionais de dimensão $21-16=5$ :

$$
\begin{aligned}
& \mathcal{V}\left(X_{11}, X_{22}, X_{33}, X_{34}, X_{43}, X_{25}, X_{42}, X_{32}, X_{52}, X_{51}, X_{23}, X_{24}, X_{21}\right. \\
& \left.X_{13} X_{31}+X_{14} X_{41}, X_{16} X_{61}, X_{13}\right)= \\
& =\mathcal{V}\left(X_{11}, X_{22}, X_{33}, X_{34}, X_{43}, X_{25}, X_{42}, X_{32}, X_{52}, X_{51}, X_{23}, X_{24}, X_{21}, X_{13}\right. \\
& \left.X_{14} X_{41}, X_{16} X_{61}\right)
\end{aligned}
$$

$$
\begin{aligned}
& \mathcal{V}\left(X_{11}, X_{22}, X_{33}, X_{34}, X_{43}, X_{25}, X_{42}, X_{32}, X_{52}, X_{51}, X_{23}, X_{24},\right. \\
& \left.X_{12} X_{21}, X_{13} X_{31}+X_{14} X_{41}, X_{16} X_{61}, X_{13}\right)= \\
& =\mathcal{V}\left(X_{11}, X_{22}, X_{33}, X_{34}, X_{43}, X_{25}, X_{42}, X_{32}, X_{52}, X_{51}, X_{23}, X_{24}, X_{13},\right. \\
& X_{12} X_{21}, X_{14} X_{41}, X_{16} X_{61}
\end{aligned}
$$

$$
\begin{aligned}
& \mathcal{V}\left(X_{11}, X_{22}, X_{33}, X_{34}, X_{43}, X_{25}, X_{42}, X_{32}, X_{52}, X_{51}, X_{23}, X_{41}, 2 X_{21} X_{31}+X_{24} X_{61},\right. \\
& \left.2 X_{12} X_{21}+2 X_{13} X_{31}+X_{16} X_{61},\left(X_{12} X_{21}+X_{13} X_{31}\right)^{2}+2 X_{12} X_{13} X_{24} X_{61}, X_{24}\right)= \\
& =\mathcal{V}\left(X_{11}, X_{22}, X_{33}, X_{34}, X_{43}, X_{25}, X_{42}, X_{32}, X_{52}, X_{51}, X_{23}, X_{41}, X_{24}, X_{21} X_{31},\right. \\
& \left.X_{12} X_{21}+X_{13} X_{31}, X_{16} X_{61}\right)= \\
& =\mathcal{V}\left(X_{11}, X_{22}, X_{33}, X_{34}, X_{43}, X_{25}, X_{42}, X_{32}, X_{52}, X_{51}, X_{23}, X_{41}, X_{24}, X_{21},\right. \\
& \left.X_{13} X_{31}, X_{16} X_{61}\right) \cup \\
& \cup \mathcal{V}\left(X_{11}, X_{22}, X_{33}, X_{34}, X_{43}, X_{25}, X_{42}, X_{32}, X_{52}, X_{51}, X_{23}, X_{41}, X_{24}, X_{31},\right. \\
& \left.X_{12} X_{21}, X_{16} X_{61}\right)
\end{aligned}
$$


então a variedade (4.56) acrescida de $X_{23}$ é equidimensional de dimensão 6 e a variedade (4.56) é equidimensional de dimensão 7.

A variedade (4.57) acrescida de $X_{13}$ é igual a:

$$
\begin{aligned}
& \mathcal{V}\left(X_{11}, X_{22}, X_{33}, X_{34}, X_{43}, X_{25}, X_{42}, X_{32}, X_{52}, X_{21}, X_{23} X_{31}+X_{24} X_{41}, X_{14} X_{23}-X_{13} X_{24}\right. \\
& \left.X_{13} X_{31}+X_{14} X_{41}+X_{15} X_{51}, X_{16} X_{61}, X_{13}\right)= \\
& =\mathcal{V}\left(X_{11}, X_{22}, X_{33}, X_{34}, X_{43}, X_{25}, X_{42}, X_{32}, X_{52}, X_{21}, X_{13}, X_{23} X_{31}+X_{24} X_{41}, X_{14} X_{23},\right. \\
& \left.X_{14} X_{41}+X_{15} X_{51}, X_{16} X_{61}\right)= \\
& =\mathcal{V}\left(X_{11}, X_{22}, X_{33}, X_{34}, X_{43}, X_{25}, X_{42}, X_{32}, X_{52}, X_{21}, X_{13}, X_{14}, X_{23} X_{31}+X_{24} X_{41},\right. \\
& \left.X_{15} X_{51}, X_{16} X_{61}\right) \cup \\
& \cup \mathcal{V}\left(X_{11}, X_{22}, X_{33}, X_{34}, X_{43}, X_{25}, X_{42}, X_{32}, X_{52}, X_{21}, X_{13}, X_{23}, X_{24} X_{41},\right. \\
& \left.X_{14} X_{41}+X_{15} X_{51}, X_{16} X_{61}\right)= \\
& =\mathcal{V}\left(X_{11}, X_{22}, X_{33}, X_{34}, X_{43}, X_{25}, X_{42}, X_{32}, X_{52}, X_{21}, X_{13}, X_{14}, X_{23} X_{31}+X_{24} X_{41},\right. \\
& \left.X_{15} X_{51}, X_{16} X_{61}\right) \cup \\
& \cup \mathcal{V}\left(X_{11}, X_{22}, X_{33}, X_{34}, X_{43}, X_{25}, X_{42}, X_{32}, X_{52}, X_{21}, X_{13}, X_{23}, X_{24},\right. \\
& \left.X_{14} X_{41}+X_{15} X_{51}, X_{16} X_{61}\right) \cup \\
& \cup \mathcal{V}\left(X_{11}, X_{22}, X_{33}, X_{34}, X_{43}, X_{25}, X_{42}, X_{32}, X_{52}, X_{21}, X_{13}, X_{23}, X_{41},\right. \\
& \left.X_{15} X_{51}, X_{16} X_{61}\right)
\end{aligned}
$$

As variedades abaixo são equidimensionais de dimensão $21-16=5$ :

$$
\begin{aligned}
& \mathcal{V}\left(X_{11}, X_{22}, X_{33}, X_{34}, X_{43}, X_{25}, X_{42}, X_{32}, X_{52}, X_{21}, X_{13}, X_{14}, X_{23} X_{31}+X_{24} X_{41},\right. \\
& \left.X_{15} X_{51}, X_{16} X_{61}, X_{24}\right)= \\
& =\mathcal{V}\left(X_{11}, X_{22}, X_{33}, X_{34}, X_{43}, X_{25}, X_{42}, X_{32}, X_{52}, X_{21}, X_{13}, X_{14}, X_{24}, X_{23} X_{31},\right. \\
& \left.X_{15} X_{51}, X_{16} X_{61}\right) \\
& \quad \\
& \quad \mathcal{V}\left(X_{11}, X_{22}, X_{33}, X_{34}, X_{43}, X_{25}, X_{42}, X_{32}, X_{52}, X_{21}, X_{13}, X_{23}, X_{24},\right. \\
& \left.\quad X_{14} X_{41}+X_{15} X_{51}, X_{16} X_{61}, X_{14}\right)= \\
& \quad=\mathcal{V}\left(X_{11}, X_{22}, X_{33}, X_{34}, X_{43}, X_{25}, X_{42}, X_{32}, X_{52}, X_{21}, X_{13}, X_{23}, X_{24}, X_{14},\right. \\
& \left.\quad X_{15} X_{51}, X_{16} X_{61}\right)
\end{aligned}
$$

então a variedade (4.57) acrescida de $X_{13}$ é equidimensional de dimensão 6 e a variedade (4.57) é equidiemnsional de dimensão 7. 
A variedade (4.58) acrescida de $X_{13}$ é igual a:

$$
\begin{aligned}
& \mathcal{V}\left(X_{11}, X_{22}, X_{33}, X_{34}, X_{43}, X_{25}, X_{42}, X_{32}, X_{52}, X_{23} X_{31}+X_{24} X_{41}, X_{14} X_{23}-X_{13} X_{24}\right. \\
& \left.X_{12} X_{21}+X_{13} X_{31}+X_{14} X_{41}+X_{15} X_{51}, X_{16} X_{61},-X_{15} X_{51}-2 X_{13} X_{31}-2 X_{14} X_{41}, X_{13}\right)= \\
& =\mathcal{V}\left(X_{11}, X_{22}, X_{33}, X_{34}, X_{43}, X_{25}, X_{42}, X_{32}, X_{52}, X_{13}, X_{23} X_{31}+X_{24} X_{41}, X_{14} X_{23},\right. \\
& \left.X_{12} X_{21}+X_{14} X_{41}+X_{15} X_{51}, X_{16} X_{61},-X_{15} X_{51}-2 X_{14} X_{41}\right)= \\
& =\mathcal{V}\left(X_{11}, X_{22}, X_{33}, X_{34}, X_{43}, X_{25}, X_{42}, X_{32}, X_{52}, X_{13}, X_{14}, X_{23} X_{31}+X_{24} X_{41},\right. \\
& \left.X_{12} X_{21}, X_{15} X_{51}, X_{16} X_{61}\right) \cup \\
& \cup \mathcal{V}\left(X_{11}, X_{22}, X_{33}, X_{34}, X_{43}, X_{25}, X_{42}, X_{32}, X_{52}, X_{13}, X_{23}, X_{24} X_{41},\right. \\
& \left.X_{12} X_{21}+X_{14} X_{41}+X_{15} X_{51}, X_{16} X_{61},-X_{15} X_{51}-2 X_{14} X_{41}\right)= \\
& =\mathcal{V}\left(X_{11}, X_{22}, X_{33}, X_{34}, X_{43}, X_{25}, X_{42}, X_{32}, X_{52}, X_{13}, X_{14}, X_{23} X_{31}+X_{24} X_{41},\right. \\
& \left.X_{12} X_{21}, X_{15} X_{51}, X_{16} X_{61}\right) \cup \\
& \cup \mathcal{V}\left(X_{11}, X_{22}, X_{33}, X_{34}, X_{43}, X_{25}, X_{42}, X_{32}, X_{52}, X_{13}, X_{23}, X_{24},\right. \\
& \left.X_{12} X_{21}+X_{14} X_{41}+X_{15} X_{51}, X_{16} X_{61},-X_{15} X_{51}-2 X_{14} X_{41}\right) \cup \\
& \cup \mathcal{V}\left(X_{11}, X_{22}, X_{33}, X_{34}, X_{43}, X_{25}, X_{42}, X_{32}, X_{52}, X_{13}, X_{23}, X_{41},\right. \\
& \left.X_{12} X_{21}, X_{15} X_{51}, X_{16} X_{61}\right)
\end{aligned}
$$

As variedades abaixo são equidimensionais de dimensão $21-16=5$

$$
\begin{aligned}
& \mathcal{V}\left(X_{11}, X_{22}, X_{33}, X_{34}, X_{43}, X_{25}, X_{42}, X_{32}, X_{52}, X_{13}, X_{14}, X_{23} X_{31}+X_{24} X_{41},\right. \\
& \left.X_{12} X_{21}, X_{15} X_{51}, X_{16} X_{61}, X_{24}\right)= \\
& =\mathcal{V}\left(X_{11}, X_{22}, X_{33}, X_{34}, X_{43}, X_{25}, X_{42}, X_{32}, X_{52}, X_{13}, X_{14}, X_{24}, X_{23} X_{31},\right. \\
& X_{12} X_{21}, X_{15} X_{51}, X_{16} X_{61} \\
& \quad \mathcal{V}\left(X_{11}, X_{22}, X_{33}, X_{34}, X_{43}, X_{25}, X_{42}, X_{32}, X_{52}, X_{13}, X_{23}, X_{24},\right. \\
& \left.\quad X_{12} X_{21}+X_{14} X_{41}+X_{15} X_{51}, X_{16} X_{61},-X_{15} X_{51}-2 X_{14} X_{41}, X_{41}\right)= \\
& \quad=\mathcal{V}\left(X_{11}, X_{22}, X_{33}, X_{34}, X_{43}, X_{25}, X_{42}, X_{32}, X_{52}, X_{13}, X_{23}, X_{24}, X_{41},\right. \\
& \left.\quad X_{12} X_{21}, X_{15} X_{51}, X_{16} X_{61}\right)
\end{aligned}
$$

então a variedade (4.58) acrescida de $X_{13}$ é equidimensional de dimensão 6 e a variedade (4.58) é equidimensional de dimensão 7.

Portanto a variedade (4.55) acrescida de $X_{52}$ é equidimensional de dimensão 7 e a variedade (4.55) é equidimensional de dimensão 8.

Assim concluimos que a variedade (4.43) é equidimensional de dimenao 8.

E a variedade $H_{3}$ é equidimensional de dimensão 8 .

Por fim, concluimos que $Z_{2}^{(5)}$ acrescida de $X_{42}$ é equidimensional de dimensão 8 e $Z_{2}^{(5)}$ é equidimensional de dimensão 9 .

(c) $Z_{3}^{(5)}$

$$
\begin{aligned}
& Z_{2}^{(5)}=\mathcal{V}\left(X_{11}, X_{22}, X_{33}, X_{34}, X_{43}, X_{25}, X_{23} X_{32}+X_{24} X_{42}, X_{23} X_{31}+X_{24} X_{41}, A, \phi_{6}^{(0)}, \phi_{4}^{(0)}, \partial_{X_{11}} \phi_{4}^{(0)}\right) \\
& Z_{3}^{(5)}=\mathcal{V}\left(X_{11}, X_{22}, X_{33}, X_{34}, X_{43}, X_{25}, X_{23} X_{32}+X_{24} X_{42}, X_{14} X_{23}-X_{13} X_{24}, A, \phi_{6}^{(0)}, \phi_{4}^{(0)}, \partial_{X_{11}} \phi_{4}^{(0)}\right)
\end{aligned}
$$

Denote por $Z_{2}^{(5)}\left(\phi_{6}^{(0)}\right)$ o elemento $\phi_{6}^{(0)}$ calculado considerando $X_{22}=X_{33}=X_{34}=X_{24}=2 X_{23} X_{32}+X_{25} X_{52}=X_{23}^{2}+X_{25} X_{43}=0$. De maneira análoga, defina $\left.Z_{3}^{(5)}(A), Z_{3}^{(5)}\left(\partial_{X_{11}} \phi_{6}^{(0)}\right), Z_{2}^{(5)} \phi_{4}^{(0)}\right)$ e $Z_{2}^{(5)}\left(\partial_{X_{11}} \phi_{4}^{(0)}\right)$. Para obter esses elementos, devemos 
calcular o determinante abaixo:

$$
\operatorname{det}\left(\begin{array}{cccccc}
u+X_{11} & X_{12} & X_{13} & X_{14} & X_{15} & X_{16} \\
X_{21} & u & X_{23} & 0 & X_{25} & X_{15} \\
X_{31} & X_{32} & u & 0 & 0 & X_{14} \\
X_{41} & X_{42} & X_{43} & u & -X_{23} & -X_{13} \\
X_{51} & X_{52} & X_{42} & -X_{32} & u & -X_{12} \\
X_{61} & X_{51} & X_{41} & -X_{31} & -X_{21} & u-X_{11}
\end{array}\right)
$$

e considerar no resultado final $2 X_{23} X_{32}+X_{25} X_{52}=X_{23}^{2}+X_{25} X_{43}=0$.

Denote por $Z_{3}^{(5)}\left(\phi_{6}^{(0)}\right)$ o elemento $\phi_{6}^{(0)}$ calculado considerando $X_{22}=X_{33}=X_{34}=X_{32}=2 X_{24} X_{42}+X_{25} X_{52}=X_{42}^{2}-X_{43} X_{52}=0$. De maneira análoga, defina $Z_{3}^{(5)}(A), Z_{3}^{(5)}\left(\partial_{X_{11}} \phi_{6}^{(0)}\right), Z_{3}^{(5)}\left(\phi_{4}^{(0)}\right)$ e $Z_{3}^{(5)}\left(\partial_{X_{11}} \phi_{4}^{(0)}\right)$. Para obter esses elementos, devemos calcular o determinante abaixo:

$$
\operatorname{det}\left(\begin{array}{cccccc}
u+X_{11} & X_{12} & X_{13} & X_{14} & X_{15} & X_{16} \\
X_{21} & u & X_{23} & X_{24} & X_{25} & X_{15} \\
X_{31} & 0 & u & 0 & X_{24} & X_{14} \\
X_{41} & X_{42} & X_{43} & u & -X_{23} & -X_{13} \\
X_{51} & X_{52} & X_{42} & 0 & u & -X_{12} \\
X_{61} & X_{51} & X_{41} & -X_{31} & -X_{21} & u-X_{11}
\end{array}\right)
$$

e considerar no resultado final $2 X_{24} X_{42}+X_{25} X_{52}=X_{42}^{2}-X_{43} X_{52}=0$.

Nos dois casos não consideramos $X_{11}=0$ porque precisamos calcular as diferenciais em $X_{11}$. Considere agora o automorfismo $f$ do anel de polinômios nas 21 variáveis $X_{i j}$ definido da seguinte forma:

$$
\begin{aligned}
& f\left(X_{11}\right)=X_{11} \quad, \quad f\left(X_{12}\right)=X_{51} \quad, \quad f\left(X_{13}\right)=X_{41} \quad, \quad f\left(X_{16}\right)=f\left(X_{14}\right)=X_{31} \\
& f\left(X_{15}\right)=X_{21} \quad, \quad f\left(X_{16}\right)=-X_{61} \quad, \quad f\left(X_{21}\right)=X_{15} \quad, \quad f\left(X_{22}\right)=X_{22} \\
& f\left(X_{23}\right)=-X_{23} \quad, \quad f\left(X_{24}\right)=X_{24} \quad, \quad f\left(X_{25}\right)=X_{25} \quad, \quad f\left(X_{33}\right)=X_{33}, f\left(X_{31}\right)=X_{14} \\
& f\left(X_{32}\right)=-X_{32} \quad, \quad f\left(X_{33}\right)=X_{33} \quad, \quad f\left(X_{34}\right)=X_{34} \quad, \quad f\left(X_{41}\right)=X_{13} \\
& f\left(X_{42}\right)=X_{42} \quad, \quad f\left(X_{43}\right)=X_{43} \quad, \quad f\left(X_{51}\right)=X_{12} \quad, \quad f\left(X_{52}\right)=X_{52} \\
& f\left(X_{61}\right)=-X_{16}
\end{aligned}
$$

Para obter $f\left(Z_{3}^{(5)}\left(\phi_{6}^{(0)}\right)\right), f\left(Z_{3}^{(5)}\left(\partial_{X_{11}} \phi_{6}^{(0)}\right)\right), f\left(Z_{3}^{(5)}\left(\phi_{4}^{(0)}\right)\right)$ e $f\left(Z_{3}^{(5)}\left(\partial_{X_{11}} \phi_{4}^{(0)}\right)\right)$, basta calcular o determinante abaixo:

$$
\operatorname{det}\left(\begin{array}{cccccc}
u+X_{11} & X_{51} & X_{41} & X_{31} & X_{21} & -X_{61} \\
X_{15} & u & -X_{23} & X_{24} & X_{25} & X_{21} \\
X_{14} & 0 & u & 0 & X_{24} & X_{31} \\
X_{13} & X_{42} & X_{43} & u & X_{23} & -X_{41} \\
X_{12} & X_{52} & X_{42} & 0 & u & -X_{51} \\
-X_{16} & X_{12} & X_{13} & -X_{14} & -X_{15} & u-X_{11}
\end{array}\right)
$$

que é o determinante (4.60) substituindo $X_{i j}$ por $f\left(X_{i j}\right)$.

Usando um programa, obtemos que (4.61) é igual a (4.59).

Assim podemos concluir que:

$$
\begin{array}{lll}
f\left(Z_{3}^{(5)}\left(\phi_{6}^{(0)}\right)\right)=Z_{2}^{(5)}\left(\phi_{6}^{(0)}\right), & & f\left(Z_{3}^{(5)}\left(\partial_{X_{11}} \phi_{6}^{(0)}\right)\right)=Z_{2}^{(5)}\left(\partial_{X_{11}} \phi_{6}^{(0)}\right) \\
f\left(Z_{3}^{(5)}\left(\phi_{4}^{(0)}\right)\right)=Z_{2}^{(5)}\left(\phi_{4}^{(0)}\right), & & f\left(Z_{3}^{(5)}\left(\partial_{X_{11}} \phi_{4}^{(0)}\right)\right)=Z_{2}^{(5)}\left(\partial_{X_{11}} \phi_{4}^{(0)}\right)
\end{array}
$$


e

$$
\begin{aligned}
& f\left(Z_{3}^{(5)}\right):=\mathcal{V}\left(f\left(X_{11}\right), f\left(X_{22}\right), f\left(X_{33}\right), f\left(X_{34}\right), f\left(X_{24}\right), f\left(X_{32}\right), f\left(X_{52}\right), f(A), f\left(\phi_{6}^{(0)}\right),\right. \\
& \left.f\left(\partial_{X_{11}} \phi_{6}^{(0)}\right), f\left(\phi_{4}^{(0)}\right), f\left(\partial_{X_{11}} \phi_{4}^{(0)}\right)\right)= \\
& =\mathcal{V}\left(X_{11}, X_{22}, X_{33}, X_{34}, X_{24}, X_{32}, X_{25}, A, \phi_{6}^{(0)}, \partial_{X_{11}} \phi_{6}^{(0)}, \phi_{4}^{(0)}, \partial_{X_{11}} \phi_{4}^{(0)}\right)=Z_{2}^{(5)}
\end{aligned}
$$

Assim concluimos que $Z_{3}^{(5)}$ e $Z_{2}^{(5)}$ são variedades isomórficas. Como $Z_{2}^{(5)}$ é equidimensional de dimensão $9 Z_{3}^{(5)}$ também é equidimensional de dimensão 9.

(v) $W_{6}$

$$
\begin{aligned}
& W_{5}=\mathcal{V}\left(X_{11}, X_{22}, X_{33}, X_{34}, X_{43}, X_{25}, X_{23} X_{32}+X_{24} X_{42}, A, \phi_{6}^{(0)}, \partial_{X_{11}} \phi_{6}^{(0)}, \phi_{4}^{(0)}, \partial_{X_{11}} \phi_{4}^{(0)}\right) \\
& W_{6}=\mathcal{V}\left(X_{11}, X_{22}, X_{33}, X_{34}, X_{43}, X_{52}, X_{23} X_{32}+X_{24} X_{42}, A, \phi_{6}^{(0)}, \partial_{X_{11}} \phi_{6}^{(0)}, \phi_{4}^{(0)}, \partial_{X_{11}} \phi_{4}^{(0)}\right)
\end{aligned}
$$

Pelo automorfismo que leva $X_{i j}$ em $X_{j i}$ concluimos que $W_{6}$ é isomórfico a $W_{5}$. Portanto, $W_{6}$ é equidimensional de dimensão 9.

Portanto, segue o teorema.

\section{Observação 4.3.1.}

$$
\begin{aligned}
W_{3}= & \mathcal{V}\left(X_{11}, X_{22}, X_{33}, X_{34}, X_{24}, 2 X_{23} X_{32}+X_{25} X_{52}, A, X_{23}^{2}+X_{25} X_{43}, \phi_{6}^{(0)}, \partial_{X_{11}} \phi_{6}^{(0)},\right. \\
& \left.\phi_{4}^{(0)}, \partial_{X_{11}} \phi_{4}^{(0)}\right)
\end{aligned}
$$

Calculando o determinante da matriz $F$ do exemplo 4.3 .3 para $X_{22}=X_{33}=X_{34}=X_{24}=2 X_{23} X_{32}+X_{25} X_{52}=X_{23}^{2}+X_{25} X_{43}=0$, temos (considerando $X_{11}=0$ ):

$$
\begin{aligned}
\partial_{X_{11}} \phi_{6}^{(0)}= & 2 X_{13} X_{25} X_{32}^{2} X_{41}-2 X_{13} X_{25} X_{31} X_{32} X_{42}+2 X_{14} X_{25} X_{32} X_{41} X_{42}- \\
& -2 X_{14} X_{25} X_{31} X_{42}^{2}+2 X_{15} X_{21} X_{32}^{2} X_{43}+2 X_{13} X_{21} X_{23} X_{32}^{2}- \\
& -2 X_{15} X_{23} X_{32}^{2} X_{41}+2 X_{14} X_{21} X_{23} X_{32} X_{42}+2 X_{15} X_{23} X_{31} X_{32} X_{42}= \\
& =2 X_{13} X_{25} X_{32}^{2} X_{41}-2 X_{13} X_{25} X_{31} X_{32} X_{42}+2 X_{14} X_{25} X_{32} X_{41} X_{42}- \\
& -2 X_{14} X_{25} X_{31} X_{42}^{2}+2 X_{15} X_{21} X_{32}^{2} X_{43}-X_{13} X_{21} X_{25} X_{32} X_{52}+ \\
& +X_{15} X_{25} X_{32} X_{41} X_{52}-X_{14} X_{21} X_{25} X_{42} X_{52}-X_{15} X_{25} X_{31} X_{42} X_{52}
\end{aligned}
$$

$\partial_{X_{11}} \phi_{6}^{(0)}$ não é fatorável em $W_{3}$, diferente do que acontece em $W_{1}$ e $W_{5}$. Assim, já partimos para o método de acrescentar variável. Apesar de várias tentativas, não foi possível provar que $W_{3}$ é equidimensional de dimensão 9.

Assim, partimos para outra abordagem. Note que:

$$
\begin{aligned}
\partial_{X_{11}} \phi_{6}^{(0)}= & X_{25}\left(X_{32} X_{41}-X_{31} X_{42}-\frac{1}{2} X_{21} X_{52}\right)\left(2 X_{13} X_{32}\right)+ \\
& +X_{25}\left(X_{32} X_{41}-X_{31} X_{42}-\frac{1}{2} X_{21} X_{52}\right)\left(2 X_{14} X_{42}\right)+ \\
& +X_{25}\left(X_{32} X_{41}-2_{31} X_{42}\right)\left(X_{15} X_{52}\right)+2 X_{15} X_{21} X_{32}^{2} X_{43}
\end{aligned}
$$

Então o monômio $+2 X_{15} X_{21} X_{32}^{2} X_{43}$ é o "problema". Um jeito de contornar esse problema é descrito 
a seguir. Considere a variedade:

$$
\begin{aligned}
& Z=\mathcal{V}\left(X_{11}, X_{22}, X_{33}, X_{34}, X_{24}, 2 X_{23} X_{32}+X_{25} X_{52}, A, X_{23}^{2}+X_{25} X_{43}, \phi_{6}^{(0)}, X_{25} \partial_{X_{11}} \phi_{6}^{(0)},\right. \\
& \left.\quad \phi_{4}^{(0)}, \partial_{X_{11}} \phi_{4}^{(0)}\right)= \\
& \quad=\mathcal{V}\left(X_{11}, X_{22}, X_{33}, X_{34}, X_{24}, X_{25}, X_{23}, A, \phi_{6}^{(0)}, \phi_{4}^{(0)}, \partial_{X_{11}} \phi_{4}^{(0)}\right) \cup W_{3}
\end{aligned}
$$

Temos:

$$
\begin{aligned}
X_{25} \partial_{X_{11}} \phi_{6}^{(0)} & =2 X_{13} X_{25}^{2} X_{32}^{2} X_{41}-2 X_{13} X_{25}^{2} X_{31} X_{32} X_{42}+2 X_{14} X_{25}^{2} X_{32} X_{41} X_{42}- \\
& -2 X_{14} X_{25}^{2} X_{31} X_{42}^{2}+2 X_{15} X_{21} X_{25} X_{32}^{2} X_{43}+2 X_{13} X_{21} X_{23} X_{25} X_{32}^{2}- \\
& -2 X_{15} X_{23} X_{25} X_{32}^{2} X_{41}+2 X_{14} X_{21} X_{23} X_{25} X_{32} X_{42}+2 X_{15} X_{23} X_{25} X_{31} X_{32} X_{42}= \\
& =2 X_{13} X_{25}^{2} X_{32}^{2} X_{41}-2 X_{13} X_{25}^{2} X_{31} X_{32} X_{42}+2 X_{14} X_{25}^{2} X_{32} X_{41} X_{42}- \\
& -2 X_{14} X_{25}^{2} X_{31} X_{42}^{2}-2 X_{15} X_{21} X_{23}^{2} X_{32}^{2}-X_{13} X_{21} X_{25}^{2} X_{32} X_{52}+ \\
& +X_{15} X_{25}^{2} X_{32} X_{41} X_{52}-X_{14} X_{21} X_{25}^{2} X_{42} X_{52}-X_{15} X_{25}^{2} X_{31} X_{42} X_{52}= \\
& =2 X_{13} X_{25}^{2} X_{32}^{2} X_{41}-2 X_{13} X_{25}^{2} X_{31} X_{32} X_{42}+2 X_{14} X_{25}^{2} X_{32} X_{41} X_{42}- \\
& -2 X_{14} X_{25}^{2} X_{31} X_{42}^{2}-\frac{1}{2} X_{15} X_{21} X_{25}^{2} X_{52}^{2}-X_{13} X_{21} X_{25}^{2} X_{32} X_{52}+ \\
& +X_{15} X_{25}^{2} X_{32} X_{41} X_{52}-X_{14} X_{21} X_{25}^{2} X_{42} X_{52}-X_{15} X_{25}^{2} X_{31} X_{42} X_{52}= \\
& =X_{25}^{2}\left(X_{32} X_{41}-X_{31} X_{42}-\frac{1}{2} X_{21} X_{52}\right)\left(2 X_{13} X_{32}+2 X_{14} X_{42}+X_{15} X_{52}\right)
\end{aligned}
$$

Então:

$$
\begin{aligned}
Z & =\mathcal{V}\left(X_{11}, X_{22}, X_{33}, X_{34}, X_{24}, X_{25}, X_{23}, A, \phi_{6}^{(0)}, \phi_{4}^{(0)}, \partial_{X_{11}} \phi_{4}^{(0)}\right) \cup \\
& \cup \mathcal{V}\left(X_{11}, X_{22}, X_{33}, X_{34}, X_{24}, 2 X_{23} X_{32}+X_{25} X_{52}, A, X_{23}^{2}+X_{25} X_{43}, \phi_{6}^{(0)}\right. \\
& \left.\left(X_{32} X_{41}-X_{31} X_{42}-\frac{1}{2} X_{21} X_{52}\right)\left(2 X_{13} X_{32}+2 X_{14} X_{42}+X_{15} X_{52}\right), \phi_{4}^{(0)}, \partial_{X_{11}} \phi_{4}^{(0)}\right)
\end{aligned}
$$

Defina:

$$
\begin{aligned}
Z_{1}^{(3)} & =\mathcal{V}\left(X_{11}, X_{22}, X_{33}, X_{34}, X_{24}, X_{25}, X_{23}, A, \phi_{6}^{(0)}, \phi_{4}^{(0)}, \partial_{X_{11}} \phi_{4}^{(0)}\right) \cap W_{3}= \\
& =\mathcal{V}\left(X_{11}, X_{22}, X_{33}, X_{34}, X_{24}, X_{25}, X_{23}, A, \phi_{6}^{(0)}, \partial_{X_{11}} \phi_{6}^{(0)}, \phi_{4}^{(0)}, \partial_{X_{11}} \phi_{4}^{(0)}\right) \\
Z_{2}^{(3)} & =\mathcal{V}\left(X_{11}, X_{22}, X_{33}, X_{34}, X_{24}, 2 X_{23} X_{32}+X_{25} X_{52}, A, X_{23}^{2}+X_{25} X_{43}, \phi_{6}^{(0)},\right. \\
& \left.X_{32} X_{41}-X_{31} X_{42}-\frac{1}{2} X_{21} X_{52}, \phi_{4}^{(0)}, \partial_{X_{11}} \phi_{4}^{(0)}\right) \cap W_{3}= \\
& =\mathcal{V}\left(X_{11}, X_{22}, X_{33}, X_{34}, X_{24}, 2 X_{23} X_{32}+X_{25} X_{52}, A, X_{23}^{2}+X_{25} X_{43}, \phi_{6}^{(0)},\right. \\
& \left.\partial_{X_{11}} \phi_{6}^{(0)}, X_{32} X_{41}-X_{31} X_{42}-\frac{1}{2} X_{21} X_{52}, \phi_{4}^{(0)}, \partial_{X_{11}} \phi_{4}^{(0)}\right) \\
Z_{3}^{(3)} & =\mathcal{V}\left(X_{11}, X_{22}, X_{33}, X_{34}, X_{24}, 2 X_{23} X_{32}+X_{25} X_{52}, A, X_{23}^{2}+X_{25} X_{43}, \phi_{6}^{(0)},\right. \\
& \left.2 X_{13} X_{32}+2 X_{14} X_{42}+X_{15} X_{52}, \phi_{4}^{(0)}, \partial_{X_{11}} \phi_{4}^{(0)}\right) \cap W_{3}= \\
& =\mathcal{V}\left(X_{11}, X_{22}, X_{33}, X_{34}, X_{24}, 2 X_{23} X_{32}+X_{25} X_{52}, A, X_{23}^{2}+X_{25} X_{43}, \phi_{6}^{(0)},\right. \\
& \left.\partial_{X_{11}} \phi_{6}^{(0)}, 2 X_{13} X_{32}+2 X_{14} X_{42}+X_{15} X_{52}, \phi_{4}^{(0)}, \partial_{X_{11}} \phi_{4}^{(0)}\right)
\end{aligned}
$$

Assim $W_{3}=Z_{1}^{(3)} \cup Z_{2}^{(3)} \cup Z_{3}^{(3)}$. Dessa forma, $W_{3}$ é equidimensional de dimensão 9 se e somente se $Z_{1}^{(3)}, Z_{2}^{(3)}$ e $Z_{3}^{(3)}$ são equidimensionais de dimensão 9.

A variedade $Z_{1}^{(3)}$ é isomórfica a $W_{1}$ : 


$$
W_{1}=\mathcal{V}\left(X_{11}, X_{22}, X_{33}, X_{34}, X_{24}, X_{32}, X_{25}, A, \phi_{6}^{(0)}, \partial_{X_{11}} \phi_{6}^{(0)}, \phi_{4}^{(0)}, \partial_{X_{11}} \phi_{4}^{(0)}\right)
$$

Denote por $W_{1}\left(\phi_{6}^{(0)}\right)$ o elemento $\phi_{6}^{(0)}$ calculado considerando $X_{22}=X_{33}=X_{34}=X_{24}=X_{32}=X_{25}$. De maneira análoga, defina $W_{1}(A), W_{1}\left(\partial_{X_{11}} \phi_{6}^{(0)}\right), W_{1}\left(\phi_{4}^{(0)}\right)$ e $W_{1}\left(\partial_{X_{11}} \phi_{4}^{(0)}\right)$. Para obter esses elementos, devemos calcular o determinante abaixo:

$$
\operatorname{det}\left(\begin{array}{cccccc}
u+X_{11} & X_{12} & X_{13} & X_{14} & X_{15} & X_{16} \\
X_{21} & u & X_{23} & 0 & 0 & X_{15} \\
X_{31} & 0 & u & 0 & 0 & X_{14} \\
X_{41} & X_{42} & X_{43} & u & -X_{23} & -X_{13} \\
X_{51} & X_{52} & X_{42} & 0 & u & -X_{12} \\
X_{61} & X_{51} & X_{41} & -X_{31} & -X_{21} & u-X_{11}
\end{array}\right)
$$

Denote por $Z_{1}^{(3)}\left(\phi_{6}^{(0)}\right)$ o elemento $\phi_{6}^{(0)}$ calculado considerando $X_{22}=X_{33}=X_{34}=X_{24}=X_{25}=X_{23}$. De maneira análoga, defina $Z_{1}^{(3)}(A), Z_{1}^{(3)}\left(\partial_{X_{11}} \phi_{6}^{(0)}\right)$, $Z_{1}^{(3)}\left(\phi_{4}^{(0)}\right)$ e $Z_{1}^{(3)}\left(\partial_{X_{11}} \phi_{4}^{(0)}\right)$. Para obter esses elementos, devemos calcular o determinante abaixo:

$$
\operatorname{det}\left(\begin{array}{cccccc}
u+X_{11} & X_{12} & X_{13} & X_{14} & X_{15} & X_{16} \\
X_{21} & u & 0 & 0 & 0 & X_{15} \\
X_{31} & X_{32} & u & 0 & 0 & X_{14} \\
X_{41} & X_{42} & X_{43} & u & 0 & -X_{13} \\
X_{51} & X_{52} & X_{42} & -X_{32} & u & -X_{12} \\
X_{61} & X_{51} & X_{41} & -X_{31} & -X_{21} & u-X_{11}
\end{array}\right)
$$

Nos dois casos não consideramos $X_{11}=0$ porque precisamos calcular as diferenciais em $X_{11}$.

Considere agora o automorfismo $f$ do anel de polinômios nas 21 variáveis $X_{i j}$ definido da seguinte forma:

$$
\begin{aligned}
& \left.f\left(X_{11}\right)=X_{11} \quad, \quad f\left(X_{12}\right)=X_{13} \quad, \quad f\left(X_{13}\right)=X_{12} \quad, \quad f\left(X_{16}\right)=X_{16}, \quad f\left(X_{21}\right)=X_{31}\right)=X_{15} \\
& f\left(X_{15}\right)=X_{14} \quad, \quad f\left(X_{16}\right)=X_{16} \quad, \quad f\left(X_{21}\right)=X_{31} \quad, \quad f\left(X_{22}\right)=X_{22} \\
& f\left(X_{23}\right)=X_{32} \quad, \quad f\left(X_{24}\right)=X_{24} \quad, \quad f\left(X_{25}\right)=X_{34} \quad, \quad f\left(X_{33}\right)=X_{33}, \quad f\left(X_{31}\right)=X_{21} \\
& f\left(X_{32}\right)=X_{23} \quad, \quad f\left(X_{33}\right)=X_{33} \quad, \quad f\left(X_{34}\right)=X_{25} \quad, \quad f\left(X_{41}\right)=X_{51} \\
& f\left(X_{42}\right)=X_{42} \quad, \quad f\left(X_{43}\right)=X_{52} \quad, \quad f\left(X_{51}\right)=X_{41} \quad, \quad f\left(X_{52}\right)=X_{43} \\
& f\left(X_{61}\right)=X_{61}
\end{aligned}
$$

Para obter $f\left(Z_{1}^{(3)}\left(\phi_{6}^{(0)}\right)\right), f\left(Z_{1}^{(3)}\left(\partial_{X_{11}} \phi_{6}^{(0)}\right)\right), f\left(Z_{1}^{(3)}\left(\phi_{4}^{(0)}\right)\right)$ e $f\left(Z_{1}^{(3)}\left(\partial_{X_{11}} \phi_{4}^{(0)}\right)\right)$, basta calcular o determinante abaixo:

$$
\operatorname{det}\left(\begin{array}{cccccc}
u+X_{11} & X_{13} & X_{12} & X_{15} & X_{14} & X_{16} \\
X_{31} & u & 0 & 0 & 0 & X_{14} \\
X_{21} & X_{23} & u & 0 & 0 & X_{15} \\
X_{51} & X_{42} & X_{52} & u & 0 & -X_{12} \\
X_{41} & X_{43} & X_{42} & -X_{23} & u & -X_{13} \\
X_{61} & X_{41} & X_{51} & -X_{21} & -X_{31} & u-X_{11}
\end{array}\right)
$$

que é o determinante (4.63) substituindo $X_{i j}$ por $f\left(X_{i j}\right)$.

Usando um programa, obtemos que (4.64) é igual a (4.62). 
Assim podemos concluir que:

$$
\begin{array}{ll}
f\left(Z_{1}^{(3)}\left(\phi_{6}^{(0)}\right)\right)=W_{1}\left(\phi_{6}^{(0)}\right), & f\left(Z_{1}^{(3)}\left(\partial_{X_{11}} \phi_{6}^{(0)}\right)\right)=W_{1}\left(\partial_{X_{11}} \phi_{6}^{(0)}\right) \\
f\left(Z_{1}^{(3)}\left(\phi_{4}^{(0)}\right)\right)=W_{1}\left(\phi_{4}^{(0)}\right), &
\end{array}
$$

$e$

$$
\begin{aligned}
& f\left(Z_{1}^{(3)}\right):=\mathcal{V}\left(f\left(X_{11}\right), f\left(X_{22}\right), f\left(X_{33}\right), f\left(X_{34}\right), f\left(X_{24}\right), f\left(X_{32}\right), f\left(X_{52}\right), f(A), f\left(\phi_{6}^{(0)}\right),\right. \\
& \left.f\left(\partial_{X_{11}} \phi_{6}^{(0)}\right), f\left(\phi_{4}^{(0)}\right), f\left(\partial_{X_{11}} \phi_{4}^{(0)}\right)\right)= \\
& =\mathcal{V}\left(X_{11}, X_{22}, X_{33}, X_{34}, X_{24}, X_{32}, X_{25}, A, \phi_{6}^{(0)}, \partial_{X_{11}} \phi_{6}^{(0)}, \phi_{4}^{(0)}, \partial_{X_{11}} \phi_{4}^{(0)}\right)=W_{1}
\end{aligned}
$$

Assim concluimos que $Z_{1}^{(3)}$ e $W_{1}$ são variedades isomórficas. Como $W_{1}$ é equidimensional de dimensão 9, $Z_{1}^{(3)}$ também é equidimensional de dimensão 9.

Assim, $W_{3}$ é equidimensional de dimensão 9 se e somente se $Z_{2}^{(3)}$ e $Z_{3}^{(3)}$ são equidimensionais de dimensão 9. 


\section{Apêndice A}

\section{Programas para calcular determinante}

Nesse trabalho foi utilizado o software de matemática Sage. A seguir estão os códigos dos programas.

Para calcular o determinante:

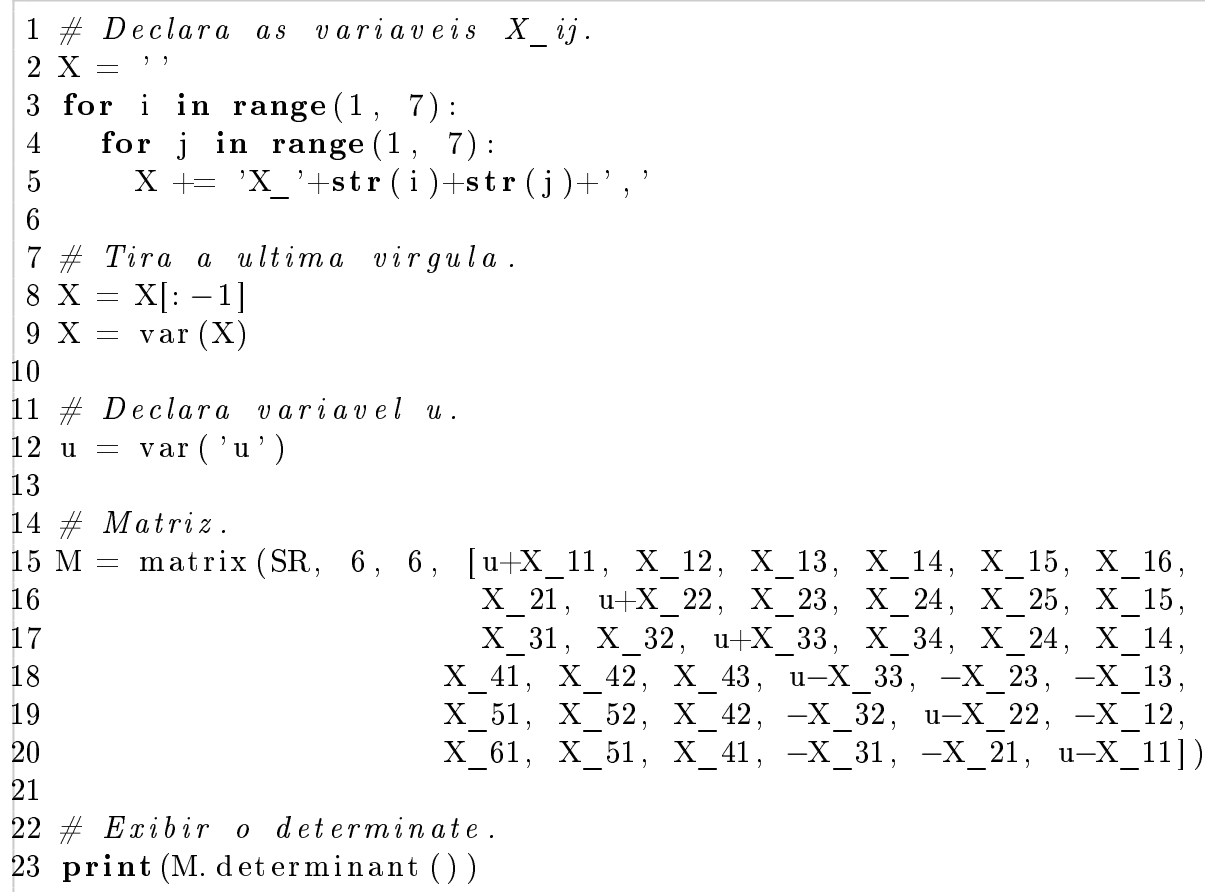

Para exibir o resultado em formato latex, trocar:

1 print(M. determinant ( ))

por

$1 \mathrm{f}=$ M. determinant ( )

2 print (latex (f))

Para calcular o determinante considerando que expressões (por exemplo $X_{23} X_{32}+X_{24} X_{42}$ e $X_{23} X_{31}+$ $\left.X_{24} X_{4} 1\right)$ :

1 \# Declara as variaveis $X_{-} i j$.

$2 \mathrm{X}=$,

3 for $i$ in range $(1,7)$ :

for $\mathrm{j}$ in range $(1,7)$ :

$\mathrm{X}+={ }^{\prime} \mathrm{X}_{-}{ }^{\prime}+\mathbf{s t r}(\mathrm{i})+\mathbf{s t r}(\mathrm{j})+,$,

7 \# Tira a ultima virgula.

$8 \mathrm{X}=\mathrm{X}[:-1]$

$9 \mathrm{X}=\operatorname{var}(\mathrm{X})$ 


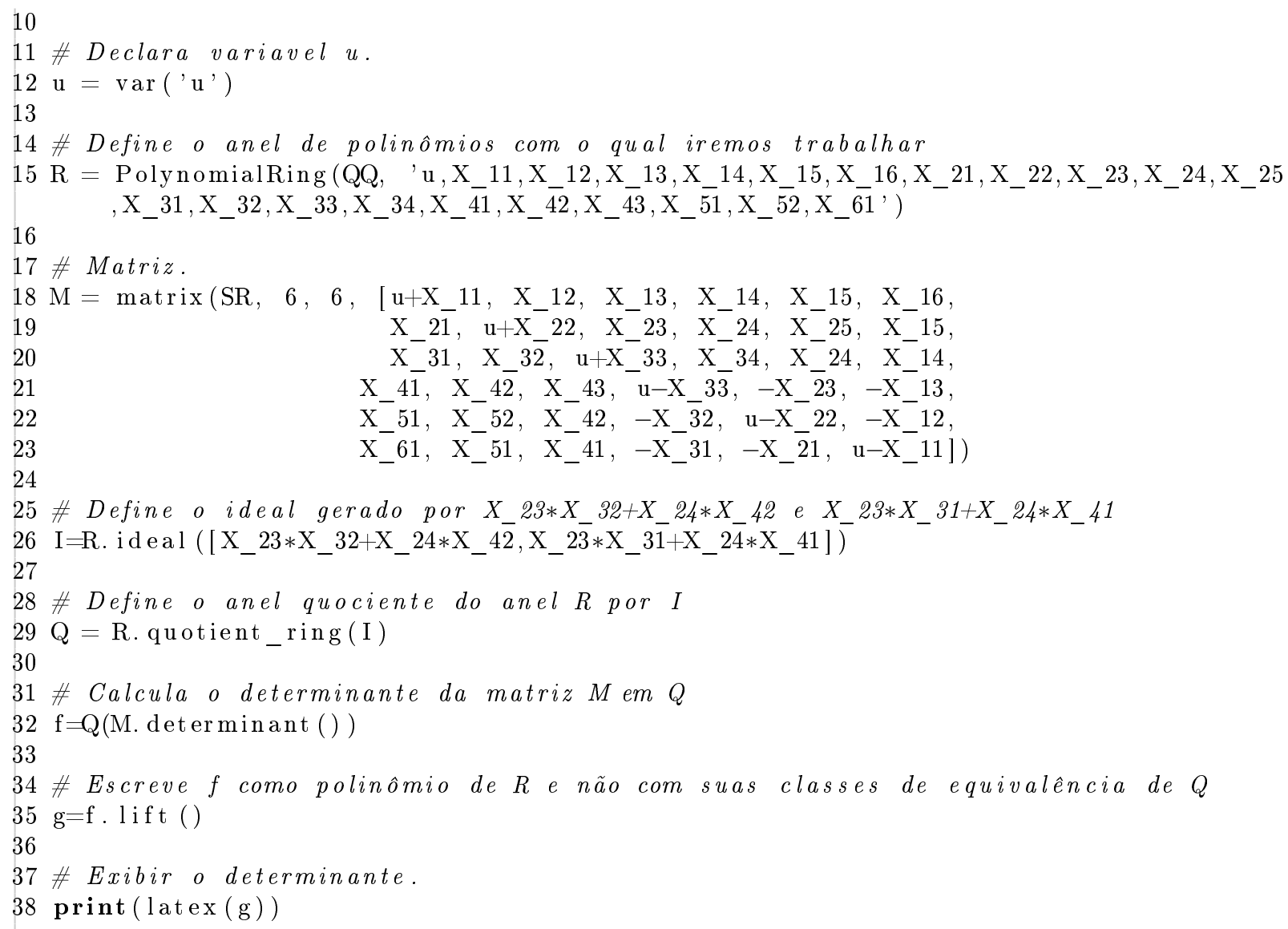




\section{Referências Bibliográficas}

Bernstein e Lunts(1996) J. Bernstein e V. Lunts. A simple proof of Kostant's theorem that $\mathrm{U}(\mathfrak{g})$ is free over its center. American Journal of Mathematics, 18(5):979-987. Citado na pág. 1

Bolsinov(1991) A. V. Bolsinov. Commutative families of functions related to consistent Poisson brackets. Acta Applicandae Mathematica, 24:253-274. Citado na pág. 20

Bourbaki(1998) N. Bourbaki. Algebra chapters 1-3. Springer. Citado na pág. 8, 9

Bourbaki(2006) N. Bourbaki. Elements de mathematique-Algebre-chapitre 10. Springer. Citado na pág. 13

Chervov e Molev(2009) A. V. Chervov e A. I. Molev. On higher-order Sugawara operators. International Mathematics Research Notices, páginas 1612-1635. Citado na pág. 25

Dixmier(1977) J. Dixmier. Enveloping algebras, volume 14. North-Holland Publishing Company. Citado na pág. 11, 20

Eisenbud(1995) D. Eisenbud. Commutative algebra, with a view toward algebraic geometry. Springer. Citado na pág. 16

Feigin e Frenkel(1992) B. Feigin e E. Frenkel. Affine Kac-Moody algebras at the critical level and Gelfand-Dikii algebras. International Journal of Modern Physics A, 7(supp01a):197-215. Citado na pág. 21

Feigin et al.(2010) B. Feigin, E. Frenkel e V. Toledano-Laredo. Gaudin models with irregular singularities. Advances in Mathematics, 223(3):873-948. Citado na pág. i, iii, 1, 20, 22, 29

Frenkel(2007) E. Frenkel. Langlands correspondence for loop groups. Cambridge University Press. Citado na pág. 21

Futorny e Molev(2015) V. Futorny e A. I. Molev. Quantization of the shift of argument subalgebras in type A. Advances in Mathematics, 285:1358-1375. Citado na pág. i, iii, 1, 19, 32

Futorny e Ovsienko(2003) V. Futorny e S. Ovsienko. Kostant's theorem for special filtered algebras. Bulletin of the London Mathematical Society, 37(2):187-199. Citado na pág. 16, 17, 37

Hartshorne(1977) R. Hartshorne. Algebraic geometry. Springer. Citado na pág. 15, 16

Humphreys(1972) J. E. Humphreys. Introduction to Lie algebras and representation theory. Springer-Verlag. Citado na pág. 4, 6, 8, 11, 14

Humphreys(1998) J. E. Humphreys. Linear algebraic groups. Springer-Verlag. Citado na pág. 14

Kostant(1963) B. Kostant. Lie group representations on polynomial rings. American Journal of Mathematics, 85(3):327-404. Citado na pág. 1

Kostrikin e Manin(1989) A. I. Kostrikin e Y. I. Manin. Linear algebra and geometry. CRC Press. Citado na pág. 8,9 
Lam(1999) T. Y. Lam. Lectures on modules and rings. Springer. Citado na pág. 16

Lang(2002) S. Lang. Algebra. Springer. Citado na pág. 7, 8

Matsumura(1989) H. Matsumura. Commutative ring theory. Cambridge University Press. Citado na pág. 16,17

Matsumura(1970) H. Matsumura. Commutative algebra. Cambridge University Press. Citado na pág. 17

Meinrenken(2013) E. Meinrenken. Clifford algebras and Lie theory. Springer. Citado na pág. 42

Mishchenko e Fomenko(1978) A. S. Mishchenko e A. T. Fomenko. Euler equations on finitedimensional Lie groups. Mathematics of the USSR-Izvestiya, 12(2):371-389. Citado na pág. 19, 20

Molev(2013) A. I. Molev. Feigin-frenkel center in types B, C and D. Inventiones mathematicae, 191(1):1-34. Citado na pág. i, iii, 1, 36, 37

Molev(2018) A. I. Molev. Sugawara operators for classical Lie algebras. American Mathematical Society. Citado na pág. 19, 23, 24, 25, 35, 36, 37, 40

Molev e Yakimova(2017) A. I. Molev e O. Yakimova. Quantisation and nilpotent limits of Mishchenko-Fomenko subalgebras. art. arXiv:1711.03917. URL https://ui.adsabs.harvard.edu/ abs/2017arXiv171103917M. Citado na pág. i, iii, 2, 39, 42

Moreau(2018) A. Moreau. A remark on Mishchenko-Fomenko algebras and regular sequences. Selecta Mathematica, 24(3):2651-2657. Citado na pág. 38

Ovsienko(2003) S. Ovsienko. Strongly nilpotent matrices and Gelfand-Zetlin modules. Linear Algebra and its Applications, 365:349-367. Citado na pág. 39

Panyushev e Yakimova(2008) D. I. Panyushev e O. S. Yakimova. The argument shift method and maximal commutative subalgebras of Poisson algebras. Mathematical Research Letters, 15(2): 239-249. Citado na pág. 20, 37

Rybnikov(2006) L. G. Rybnikov. The argument shift method and the Gaudin model. Functional Analysis and Its Applications, 40(3):188-199. Citado na pág. 1, 20, 29

Tarasov(2002) A. A. Tarasov. The maximality of certain commutative subalgebras in the Poisson algebra of a semisimple Lie algebra. Russian Mathematical Surveys, 57(5):1013-1014. Citado na pág. 20

Tauvel e Yu(2005) P. Tauvel e R.W.T. Yu. Lie algebras and algebraic groups. Springer. Citado na pág. 14,15

Vinberg(2014) E. B. Vinberg. Limits of integrable Hamiltonians on semisimple Lie algebras. Functional Analysis and Its Applications, 48(2):107-115. Citado na pág. 39

Vinberg(1991) E. B. Vinberg. On certain commutative subalgebras of a universal enveloping algebra. Mathematics of the USSR-Izvestiya, 36(1):1-22. Citado na pág. 1, 20

Zariski e Samuel(1960) O. Zariski e P. Samuel. Commutative algebra, volume 2. D. Van Nostrand Company, Inc. Citado na pág. 14

Zelobenko(1973) D. P. Zelobenko. Compact Lie groups and their representations. American Mathematical Society. Citado na pág. 39 